\title{
EMBEDDED SURFACES AND THE STRUCTURE OF DONALDSON'S POLYNOMIAL INVARIANTS
}

\author{
P. B. KRONHEIMER \& T. S. MROWKA
}

\section{Introduction}

Over the past decade, beginning with the fundamental work of Donaldson, it has become ever more clear that many aspects of the differential topology of smooth 4-manifolds can be seen as developing from a rich analogy with the complex geometry of algebraic surfaces. The canonical class, the first Chern class of the complex cotangent bundle, plays a fundamental role in the study of complex surfaces because it is the simplest invariant that one can obtain from the complex structure. The canonical class determines the genus of all smooth complex curves $C$ in a complex surface $X$ in terms of their homology classes, through the adjunction formula:

$$
2 \text { genus }(C)-2=C \cdot C+K_{X} \cdot C .
$$

In this paper, we strengthen the analogy between the smooth and complex geometries by showing that for a large class of 4-manifolds (the manifolds of simple type as defined below), the smooth structure alone determines a collection of two-dimensional cohomology classes. These 'basic classes' of the 4-manifold constrain the genus of smoothly embedded 2-manifolds by an inequality analogous to the adjunction formula. If the 4-manifold is also an algebraic surface, the canonical class is often one of the basic classes, and in many cases the inequality for the genus of embedded 2-manifolds establishes, for example, that a smooth algebraic curve achieves the smallest possible genus amongst all embedded 2-manifolds in its homology class.

Received July 20, 1994. First author supported by an Advanced Fellowship from the EPSRC. Second author partially supported by NSF/NYI grant number DMS-9357641 and a Sloan Fellowship. 
At the centre of Donaldson's programme are the polynomial invariants, which are invariants of smooth 4-manifolds constructed from the moduli spaces of anti-self-dual connections. Since their introduction, they have remained in many respects mysterious, despite their considerable success in settling some of the fundamental problems in 4dimensional topology. For each 4-manifold, there is an infinite family of invariants that one might try to compute, and it has not been clear to what extent they are independent or what aspects of the geometry or topology of the 4-manifold they reflect. Our main result reveals that, taken together, the polynomial invariants reveal a surprising amount of regularity. The total content of the invariants is encapsulated in a finite set of cohomology classes (the basic classes mentioned above) and a corresponding set of rational coefficients. Once this structure is understood, the adjunction inequality can be interpreted as constraining the basic classes, and hence the polynomial invariants, in terms the genus of all the embedded 2-manifolds in the 4-manifold.

In the remaining parts of this introduction, we give a precise statement of the main theorems, illustrate these with some examples, and give an outline of the paper. Most of the results presented here were first announced in [28].

(i) Statement of results. Throughout this paper, $X$ will denote a smooth, closed oriented 4-manifold, $Q$ (or $Q_{X}$ ) will denote the intersection form, regarded as a quadratic form on the second homology, $b_{i}(X)$ will denote $i^{\text {th }}$ Betti number and $b^{+}(X)$ the dimension of a maximal positive subspace for $Q$. As usual, following [10], we define a homology-orientation of $X$ to be an orientation of $H^{+} \oplus H^{1}(X ; \mathbb{R})$, where $H^{+}$is any such maximal positive subspace. A 4-manifold $X$ equipped with a homology-orientation will be called an admissible 4manifold if $b^{+}(X)>1$ and $b^{+}(X)-b_{1}(X)$ is odd.

Recall that given an admissible 4-manifold $X$ and a line-bundle $w$ on $X$ Donaldson constructs the polynomial invariant $q_{k, w}$ from the moduli space of anti-self-connections based on a rank-2 bundle $E$ with $\operatorname{det}(E)=w$ and $c_{2}(E)-\frac{1}{4} c_{1}(E)^{2}=k$. As originally defined [10], it is a symmetric multilinear function on the second homology $H_{2}(X)$, of degree

$$
4 k-\frac{3}{2}\left(b^{+}-b_{1}+1\right) .
$$


In this paper, we find it convenient to index the invariants by the dimension of the moduli space, which is an integer $\delta$, subject to the constraint

$$
\delta \equiv-2 w^{2}-3\left(b^{+}-b_{1}+1\right)(\bmod 8) .
$$

(Of course, the Donaldson invariants depend only on the isomorphism class of $w$, which is determined by its first Chern class. Accordingly, we will often use notation which confuses $w$ with $c_{1}(w)$, for example by writing $w^{2}$ for $c_{1}(w)^{2}$, as above, and writing the tensor product additively.) Although our main concern is the behaviour of the polynomial invariants on the two-dimensional homology, it is important to have at our disposal the natural extension of these invariants to all the homology of $X$, where they are multilinear functions, symmetric in the even dimensional homology and antisymmetric in the odd dimensional homology. We introduce the notation $\mathbb{A}(X)$ for the algebra

$$
\mathbb{A}(X)=\operatorname{Sym}\left(H_{\text {even }}(X ; \mathbb{R})\right) \otimes \Lambda\left(H_{\text {odd }}(X ; \mathbb{R})\right)
$$

where Sym denotes the symmetric tensor algebra and $\Lambda$ the exterior algebra. This is the quotient of the tensor algebra of $H_{*}(X ; \mathbb{R})$ by the ideal generated by the graded anti-commutators. We will usually write $x$ to denote the positive integral generator of $H_{0}(X)$. We make $\mathbb{A}(X)$ a graded algebra, graded by $\mathbb{Z}$, with

$$
H_{i}(X) \subset \mathbb{A}_{4-i}(X)
$$

Thus the element $x$, for example, has degree 4 . The polynomial invariant which is usually thought of as a polynomial of degree $\delta / 2$ on $H_{2}(X)$ is extended to define a linear function on $\mathbb{A}_{\delta}(X)$. Because the second homology is not our only concern, we break with traditional usage and refer to this as the polynomial invariant of degree $\delta$.

We combine the invariants of all degrees into one linear function, simply by summing over $\delta$. For us, therefore, the Donaldson invariant is a linear function on $\mathbb{A}(X)$, which we denote by

$$
D_{X}^{w}: \mathbb{A}(X) \rightarrow \mathbb{R}
$$


By definition, $D_{X}^{w}$ is zero on elements of $\mathbb{A}(X)$ of degree $\delta$ unless $\delta$ satisfies (1.2). The definition of $D_{X}^{w}$ is given in more detail in section 2.

Definition 1.4. An admissible 4-manifold $X$ has simple type if for all line-bundles $w$ and all $z \in \mathbb{A}(X)$ we have

$$
D_{X}^{w}\left(x^{2} z\right)=4 D_{X}^{w}(z)
$$

The manifolds of simple type include algebraic complete intersections in $\mathbb{C P}^{N}$, many branched coverings of algebraic surfaces, and any complex surface defined over $\mathbb{R}$ whose real locus has an orientable component of genus 2 or more. Some criteria for simple type are given in section 8 . At the time of writing, there are no examples of admissible 4-manifolds which have been shown not to have simple type.

For a manifold $X$ of simple type and $h \in H_{2}(X)$ we make the definition

$$
\begin{aligned}
\mathscr{D}^{w}(h) & =D_{X}^{w}\left(e^{h}+\frac{1}{2} x e^{h}\right) \\
& =\sum D_{X}^{w}\left(h^{d}\right) / d !+\frac{1}{2} \sum D_{X}^{w}\left(x h^{d}\right) / d ! .
\end{aligned}
$$

We make no assertion yet as to whether these series converge: we can regard them as formal functions on $H_{2}(X ; \mathbb{R})$, i.e. as elements of the ring of formal power series $\mathbb{R}\left[\left[H_{2}(X)^{*}\right]\right]$. Because $D_{X}^{w}$ is non-zero on elements of degree $\delta$ only when $\delta$ satisfies (1.2), the non-zero terms in the two series on the right-hand side involve different values of $d$ : so, from the Taylor series of $\mathscr{D}^{w}$, one can extract both $D_{X}^{w}\left(h^{d}\right)$ and $D_{X}^{w}\left(x h^{d}\right)$, for all $h$ and $d$. From the relation in Definition 1.4, one can then obtain $D_{X}^{w}\left(x^{r} h^{d}\right)$ for all $r$. Thus if $X$ has $b_{1}=0$ as well as having simple type, no information is lost in passing from $D_{X}^{w}$ to $\mathscr{D}^{w}$. Note that $\mathscr{D}^{w}$ is an even or odd formal power series according to the parity of

$$
-w^{2}-\frac{3}{2}\left(b^{+}-b_{1}+1\right)(\bmod 2) .
$$

Part of the content of the main theorem of this paper is that $\mathscr{D}^{w}$ is actually an analytic function on $H_{2}(X ; \mathbb{R})$ when $b_{1}=0$ : 
Theorem 1.7. Let $X$ be an admissible 4-manifold of simple type with $b_{1}=0$. Suppose that some Donaldson invariant of $X^{\prime}$ is non-zero. Then:

(a) There exist finitely many cohomology classes

$$
K_{1}, \ldots, K_{s} \in H^{2}(X ; \mathbb{Z})
$$

and non-zero rational numbers $\beta_{1}, \ldots, \beta_{s}$ such that

$$
\mathscr{D}^{w}=\exp \left(\frac{Q}{2}\right) \sum_{r=1}^{s}(-1)^{\left(w^{2}+K_{r} \cdot w\right) / 2} \beta_{r} e^{K_{r}}
$$

as analytic functions on $H_{2}(X ; \mathbb{R})$. Here $Q$ is the intersection form, regarded as a quadratic function. Each of the classes $K_{r}$ is an integral lift of $w_{2}(X)$.

(b) If $\Sigma_{S}$ is a smoothly embedded, oriented surface in $X$ representing a non-trivial homology class $S$ with $Q(S) \geq 0$, then the genus of $\Sigma_{S}$ satisfies the lower bound

$$
2 \operatorname{genus}\left(\Sigma_{S}\right)-2 \geq Q(S)+K_{r}(S)
$$

for all $r$.

We call the classes $K_{r}$ the basic classes of $X$.

We make some remarks to clarify a few points. Since the function $\mathscr{D}^{w}(h)$ is always either an even or an odd function of $h$, the linear combination of exponentials in Theorem 1.7 is a linear combination of either hyperbolic cosines or hyperbolic sines respectively. It follows that $-K_{r}$ is a basic class whenever $K_{r}$ is, so the term $K_{r}(S)$ in the inequality of part $(b)$ could be replaced by its absolute value. In this sense then, the lower bound for genus $\left(\Sigma_{S}\right)$ given here is in general stronger than [26]. Also notice that the theorem implies that knowledge of $\mathscr{D}^{w}$ for any one $w$ determines $\mathscr{D}^{w^{\prime}}$ for all $w^{\prime}$ on $X$.

(ii) Examples.

Example 1. Let $X$ be a $K 3$ surface, for example a smooth quartic surface in $\mathbb{C P}^{3}$. The invariants of this 4-manifold, which have degree $4 \bmod 8$, are well known: see [37] for example. In terms of the series $\mathscr{D}^{w}$, we can express the result as

$$
\mathscr{D}^{w}=(-1)^{w^{2} / 2} \exp \left(\frac{Q}{2}\right) .
$$


We will give a complete derivation of this formula in section 7 , using the techniques of this paper. In this example there is just one basic class, $K=0$, which is also the canonical class of $X$. Part $(b)$ of Theorem 1.7 then says that

$$
2 \text { genus }\left(\Sigma_{S}\right)-2 \geq Q(S)
$$

whenever $Q(S) \geq 0$ and $S \neq 0$, which reproduces the earlier result of [26].

Example 2. Let $X$ be a smooth quintic surface in $\mathbb{C P}^{3}$ and let $w$ be 0 (the trivial line bundle). This $X$ is a smooth, simply-connected 4-manifold with Euler number 55 and signature 35; its polynomial invariants are odd functions on $H_{2}(X)$ when $w=0$. As a complex surface, it has general type, and its canonical class $K_{X}$ is the Poincaré dual of the hyperplane section. This class has square 5 , from which one sees that it is primitive (that is, it is not an integer multiple of a smaller class). According to a general result of Friedman and Morgan [17], the polynomial invariants of $X$ are polynomials in $Q$ and $K_{X}$ only, from which it follows that all basic classes are (integer) multiples of $K_{X}$ and that

$$
\mathscr{D}^{0}=\exp \left(\frac{Q}{2}\right) \sum_{n} \beta_{n} \sinh \left(n K_{X}\right)
$$

(a finite sum). A further restriction on the basic classes comes from Theorem 1.7(b). In $X$ we can find smooth complex curves $C$ with $K_{X}(C)$ positive (such as a hyperplane section), and the genus of these is given by the adjunction formula (1.1). If $n K_{X}$ were a basic class for any $n>1$ then the genus of $C$ would not satisfy the lower bound of part $(b)$. It follows that $\pm K_{X}$ are the only basic classes and that $\beta_{1}$ is the only non-zero coefficient in the above sum. Using recent results of Fintushel and Stern [14], it is possible to calculate $\beta_{1}$ : we have

$$
\mathscr{D}^{0}=8 \exp \left(\frac{Q}{2}\right) \sinh \left(K_{X}\right) .
$$

Knowing $\beta_{1}$, we can also write down the invariants for non-trivial $w$ : when $w=K_{X}$, for example, we have

$$
\mathscr{D}^{w}=-8 \exp \left(\frac{Q}{2}\right) \cosh \left(K_{X}\right) .
$$

In this example, the content of Theorem 1.7(b) is that

$$
2 \text { genus }\left(\Sigma_{S}\right)-2 \geq Q(S)+\left|K_{X}(S)\right|
$$


whenever $Q(S) \geq 0$ and $[S] \neq 0$. In particular, a smooth complex curve in $X$ with non-negative self-intersection number realises the smallest possible genus in its homology class.

Example 3. In section 7, we shall calculate the invariants for the connected sum $\tilde{X}=X \# \overline{\mathbb{C P}}^{2}$ when $X$ is a $K 3$ surface. For any $w$ which is trivial on $\widetilde{\mathbb{C P}}^{2}$, we have

$$
\mathscr{D}^{w}=(-1)^{w^{2} / 2} \exp \left(\frac{Q_{\tilde{X}}}{2}\right) \cosh E,
$$

where $E$ is the generator of $H^{2}\left(\overline{\mathbb{C P}}^{2} ; \mathbb{Z}\right)$. We can write

$$
H^{2}(\tilde{X} ; \mathbb{R})=H^{2}(X ; \mathbb{R}) \oplus \mathbb{R} \cdot[E],
$$

and in these terms we have

$$
\tilde{Q}=Q-E^{2} .
$$

So the formula (1.8) can be rewritten as

$$
\mathscr{D}_{\tilde{X}}^{w}=\mathscr{D}_{X}^{w} \exp \left(-E^{2} / 2\right) \cosh E .
$$

In fact this result is not special to $K 3$ : we shall prove the following result in section 9 , which also treats the case of a line bundle $\tilde{w}=w+E$ which is non-zero on $\overline{\mathbb{C P}}^{2}$. (Here we regard $E$ as a cohomology class also, via Poincaré duality.) Of course, the second case can be deduced from the first on the basis of Theorem 1.7.

Proposition 1.9. Let $X$ be a 4-manifold of simple type with $b_{1}=0$, and let $w \rightarrow X$ be a line bundle. Let $\tilde{X}=X \# \mathbb{C P}^{2}$, and let $E$ be a generator of $H^{2}\left(\overline{\mathbb{P}}^{2} ; \mathbb{Z}\right)$. Then $\tilde{X}$ has simple type, and

$$
\begin{aligned}
\mathscr{D}_{\bar{X}}^{w} & =\mathscr{D}_{X}^{w} \exp \left(-E^{2} / 2\right) \cosh E \\
\mathscr{D}_{\tilde{X}}^{w+E} & =-\mathscr{D}_{X}^{w} \exp \left(-E^{2} / 2\right) \sinh E .
\end{aligned}
$$

In particular, if the basic classes of $X$ are $K_{r}$, then the basic classes of $\tilde{X}$ are $K_{r} \pm E$.

This result provides examples of complex surfaces where the basic classes are not just multiples of the canonical class. If $X$ is complex then so is $\tilde{X}$, and its canonical class is $K_{X}+E$ (when $E$ is oriented so 
that it is the Poincare dual of the exceptional curve with its complex orientation). The basic classes, on the other hand, also include $K_{X}-E$.

In the definition of $\mathscr{D}^{w}$ in (1.5), the way in which the point-class $x$ has been introduced deserves comment. If $X$ has simple type, we can define

$$
\begin{aligned}
{ }^{+} D^{w}(z) & =D_{X}^{w}\left(\left(1+\frac{1}{2} x\right) z\right) \\
{ }^{-} D^{w}(z) & =D_{X}^{w}\left(\left(1-\frac{1}{2} x\right) z\right),
\end{aligned}
$$

so that

$$
D_{X}^{w}=\frac{1}{2}\left({ }^{+} D^{w}+{ }^{-} D^{w}\right) .
$$

These two constituents of $D_{X}^{w}$ will then satisfy

$$
\begin{gathered}
{ }^{+} D^{w}(x z)=2^{+} D^{w}(z) \\
{ }^{-} D^{w}(x z)=-2^{-} D^{w}(z) .
\end{gathered}
$$

Because $D_{X}^{w}$ is non-zero only in degrees $\delta$ satisfying (1.2), we can recover ${ }^{-} D^{w}$ (and hence $D_{X}^{w}$ ) from ${ }^{+} D^{w}$ alone: we have

$$
{ }^{-} D^{w}(z)=e^{-2 \pi i \delta_{0} / 8+} D^{w}(\epsilon z),
$$

where $\delta_{0}$ is the right-hand side of (1.2) and $\epsilon$ is the automorphism of $\mathbb{A}(X)$ given by multiplication by $e^{2 \pi i \delta / 8}$ on $\mathbb{A}_{\delta}(X)$. The definition of $\mathscr{D}^{w}$ can be written in terms of ${ }^{+} D^{w}$ as

$$
\mathscr{D}^{w}(h)={ }^{+} D^{w}\left(e^{h}\right) .
$$

There is an alternative way to build in the point class $x$. In the case that $b_{1}=0$, we can exponentiate a general element of $H_{0}(X) \oplus H_{2}(X)$, and consider

$$
D_{X}^{w}\left(e^{h+\lambda x}\right)
$$

for $\lambda \in \mathbb{R}$ (or a formal parameter). In the simple type case, we have

$$
2 D_{X}^{w}\left(e^{h+\lambda x}\right)=e^{2 \lambda+} D^{w}\left(e^{h}\right)+e^{-2 \lambda}-D\left(e^{h}\right)
$$


so Theorem 1.7 tells us that

$$
\begin{aligned}
2 D_{X}^{w}\left(e^{h+\lambda x}\right)=e^{2 \lambda} & \exp \left(\frac{Q(h)}{2}\right) \\
& \times \sum_{r=1}^{s}(-1)^{\left(w^{2}+K_{r} \cdot w\right) / 2} \beta_{r} \exp \left(K_{r} \cdot h\right) \\
& +e^{-2 \pi i \delta_{0} / 8} e^{-2 \lambda} \exp \left(\frac{-Q(h)}{2}\right) \\
& \times \sum_{r=1}^{s}(-1)^{\left(w^{2}+K_{r} \cdot w\right) / 2} \beta_{r} \exp \left(i K_{r} \cdot h\right) .
\end{aligned}
$$

(iii) Outline. Section 2 of this paper gathers together some of the established techniques and results from gauge theory which are involved in the proof of the theorem. Our main tools are first, the moduli spaces of anti-self-dual connections with singularities in codimension-two, and second, the moduli spaces of finite-action anti-self-dual connections on manifolds with cylindrical ends, in the case that the cross-section of the cylindrical end is a circle-bundle over a Riemann surface. The necessary technical results concerning singular connections are taken from [26] and [27]. We recall in section 2(v) how such connections can be used to define invariants of a pair $(X, \Sigma)$, consisting of a suitable 4-manifold and an embedded 2-dimensional surface.

The proof of Theorem 1.7 is contained in sections 3 through 7 . The focus of our attention in sections 3,4 and 5 are the cylindrical end moduli spaces. Our aim here is to develop a limited theory of relative invariants which allows the calculation of the invariants $D_{X}^{w}$ by pairing invariants associated to the two pieces when $X$ is cut along a circle bundle. In a suitable regime, we shall show that the relative invariants have a naive form in the case that the degree of the circle bundle and the genus of the Riemann surface are both large - large, that is, in comparison with the dimensions of the moduli spaces involved. These naive relative invariants take values in the ordinary homology or cohomology of the space of irreducible flat connections on the circle bundle. We shall define the invariants without making abstract perturbations of the anti-self-duality equations, and this necessitates having a good understanding of some transversality issues for moduli spaces on cylinders. To this end, we undertake a detailed study of some aspects of these moduli spaces in section 3, building on recent results of Guo [20]. 
The relative invariants and pairing properties are then developed in section 4 .

In section 5 we apply the relative invariants to establish the central technical result of this paper. We show that the invariants of a pair $(X, \Sigma)$, defined using the moduli spaces of singular anti-self-dual connections, can, in a certain range, be expressed by universal formulae in terms of the ordinary invariants $D_{X}^{w}$. The universal formulae involve only the invariants $D_{X}^{w}$, the genus and self-intersection number of $\Sigma$, and the map on homology, $\iota_{*}: H_{*}(\Sigma) \rightarrow H_{*}(X)$, induced by the inclusion. The simplest of these universal formulae goes back to [27] (essentially Theorem 5.10 of that paper). We cannot calculate these formulae in full, but we can calculate a leading term. In some low-dimensional cases, the leading term gives the whole formula. These calculations are carried out in section 6 , after reducing them to a problem involving holomorphic bundles on a ruled surface, rather as in [27].

The moduli space of singular anti-self-dual connections on $(X, \Sigma)$ involve a choice of a real parameter $\alpha \in\left(0, \frac{1}{2}\right)$, which specifies the conjugacy class of the limiting holonomy on the small circles linking the surface. Our convention is that the holonomy on the associated $\mathrm{SO}(3)$ bundle is a rotation $\rho(\alpha) \in \mathrm{SO}(3)$ through angle $4 \pi \alpha$. The invariants of the pair are independent of $\alpha$, and this gives rise to a symmetry, or relation, among these invariants, tied to the fact that the rotations $\rho(\alpha)$ and $\rho\left(\frac{1}{2}-\alpha\right)$ are conjugate. This 'flip' symmetry was the main mechanism in [27], and was discussed again in [25].

The universal formulae of section 5 do not respect the flip symmetry: they are proved by considering a limiting situation when $\alpha$ is close to zero. So when these formulae are used to express the invariants of $(X, \Sigma)$ in terms of the ordinary invariants, the flip gives rise to nontrivial universal relations among values of $D_{X}^{w}$. These relations depend on the genus of $\Sigma$ and the homology class of the embedding, but are otherwise independent of $X$.

The universal relations are described in general terms at the beginning of section 7. At this point, because we do not know the relations in full (we only know of their existence, on account of the material in section 5), it might look as if they would be of little use to us. It turns out, however, that the limited information garnered in section 6 is sufficient to get us started. Knowing only of the existence of such relations, we are then able to calculate the entire invariant of a $K 3$ 
surface, and of a multiple blow-up of $K 3$.

In the case of a manifold $X$ of simple type with $b_{1}=0$, the general shape of the universal relations becomes much simpler, and they have the following consequence (see Corollary 7.22 for a precise statement). Let $S$ be an integral homology class in $X$ represented by a surface $\Sigma_{S}$, and consider the power-series in $t$ given by

$$
f(t)=\mathscr{D}^{w}(t S) .
$$

Under some mild constraints on $S$ and $\Sigma_{S}$ (in particular, $Q(S)$ needs to be large, for example), the universal relations imply that there are linear relations on the Taylor coefficients of $f(t)$, which determine $f(t)$ completely in terms of its Taylor coefficients of orders up to and including $t^{a}$, where

$$
a=a\left(\Sigma_{S}\right)=2 \operatorname{genus}\left(\Sigma_{S}\right)-2-Q(S) .
$$

These linear relations depend only on genus $\left(\Sigma_{S}\right)$ and $Q(S)$. Although we do not know what these linear relations are at the outset, we have in $K 3 \# k\left(\overline{\mathbb{C P}}^{2}\right)$ a rich supply of embedded surfaces of any required genus and self-intersection number, and for each of these we know the power series $f(t)$. On this basis, we are able to deduce what the consequences of the linear relations must be, and Theorem 1.7 then follows without much difficulty.

The reader who wishes to understand the proof of the main theorem without going into the more technical aspects of the gauge theory can get by with reading section 2 (excluding the material on cylindricalend moduli spaces), the statement of Theorem 5.10, which describes the nature of the universal formulae for the invariants of a pair, and section 7 , occasionally referring back to results of particular calculations in section 6 .

The last sections of this paper are not concerned with the proof of Theorem 1.7, but discuss various related issues which emerge. In section 8 we discuss the simple type property, and establish some sufficient conditions. Section 9 collects various odds and ends, beginning with a proof of the blow-up formulae for manifolds of simple type (Proposition 1.9 above). We also investigate in section 9 the relationship between Theorem 1.7 and the earlier results of [25]: both yield an obstruction to embedding surfaces of a given genus, and we consider 
conditions under which the two obstructions are the same. We finally return to algebraic surfaces, to discuss when it can be shown that the canonical class is among the basic classes, and we mention some open questions. There is an appendix on orientations of moduli spaces.

The proof of Theorem 1.7 has changed slightly since the authors published an announcement and a sketch of the proof in [28]. In particular, in the present proof, we have no need to appeal to existing calculations of invariants of elliptic surfaces. (The new trick is contained in Lemma 7.29, which is the essential device in determining the blow-up formulae.) A slight variation of the original ideas has also allowed us to derive the relation between $D_{X}^{w}$ for different values of $w$ (as expressed by the main theorem); this part of the theorem is new.

Since the publication of [28], an alternative derivation of part $(a)$ of Theorem 1.7 (for simply-connected 4-manifolds) has been given by Fintushel and Stern [14]. The overall scheme of proof is similar, but the universal relations are derived using embedded spheres rather than embedded surfaces of large genus. The technical results of section 3 , which here require the genus to be large, are replaced by results concerning instantons on cylinders based on circle bundles over spheres, which are derived from the ADHM description of instantons on $\mathbb{R}^{4}$ and its cyclic quotients. The blow-up formula (1.9) has also been derived by Lisca [31], for manifolds containing nuclei.

\section{Preliminaries}

(i) Spaces of connections. We begin with a review of the definition of Donaldson's polynomial invariants. It is important for our work to have at our disposal the case that $X$ is non-simply connected. Most of the required material is scattered in the literature so we gather the relevant points together here.

If $\lambda$ is a real line bundle then $\lambda$ defines a system of local coefficients on $X$ and we let $H^{i}(X ; \lambda)$ denote the corresponding cohomology groups. The product $\lambda \otimes \lambda$ has a canonical orientation so, as $X$ is oriented, there is a pairing $H^{2}(X ; \lambda) \otimes H^{2}(X ; \lambda) \rightarrow H^{4}(X ; \mathbb{R}) \equiv \mathbb{R}$. We let $b^{+}(\lambda)$ and $b^{-}(\lambda)$ denote the dimensions of maximal positive and negative subspaces for this quadratic form, reserving the notation $b^{+}$or $b^{+}(X)$ for the case $\lambda=\mathbb{R}$. 
Recall that a homology orientation, $o$, is an orientation of $H^{+}(X) \oplus$ $H^{1}(X)$.

Definition 2.1. A 4-manifold $X$, equipped with an orientation and a homology-orientation, is called admissible if it has $b^{+}(X)-b^{1}(X)$ odd and $b^{+}(X)>1$.

The setting that we use for gauge theory on an admissible 4-manifold is the following. Fix a $C^{\infty}$ line bundle $w$ and a unitary 2-plane bundle $E \rightarrow X$ with an isomorphism

$$
\psi: \operatorname{det}(E) \rightarrow w .
$$

We use $c$ to denote the combination of Chern numbers

$$
c(E)=\left\langle c_{2}(E)-\frac{1}{4} c_{1}^{2}(E),[X]\right\rangle
$$

and write

$$
\kappa=c(E) .
$$

Let $\mathfrak{g}_{E}$ denote the bundle of traceless skew-Hermitian endomorphisms of $E$. The gauge group $\mathscr{G}=\mathscr{G}_{E}$ of determinant one unitary automorphisms of $E$ (i.e. those that respect $\psi$ ) acts in a natural way on $\mathfrak{g}_{E}$. Let $\mathscr{A}=\mathscr{A}_{E}$ denote the space of connections in $\mathfrak{g}_{E}$. Then $\mathscr{G}$ acts on $\mathscr{A}$ and the quotient is denoted by $\mathscr{B}=\mathscr{B}_{\kappa}^{w}=\mathscr{B}_{\kappa}^{w}(X)$. For a connection $A$ in $\mathfrak{g}_{E}$, we write $c(A)$ for the Chern-Weil integral

$$
c(A)=\frac{1}{32 \pi^{2}} \int \operatorname{tr}\left(F_{A} \wedge F_{A}\right),
$$

even when the base manifold is non-compact, so that on the closed manifold $X$ we have $c(A)=c(E)$. (The trace in this formula is the trace on 3-by-3 matrices.)

We can also think of $\mathscr{B}_{\kappa}^{w}(X)$ as a space of connections in $E$ all inducing the same connection in $\operatorname{det}(E)$. When working with holomorphic bundles, we will often pass back and forth between these points of view. We will not be explicit about which topology we use on the spaces of connections and gauge groups. For most of this section we could take $\mathscr{A}$ to be the space of $L_{k}^{p}$ connections for some $p, k$ with $1<p<\infty$ and $p(k+1)>4$, and $\mathscr{G}$ to be the space of $L_{k+1}^{p}$ gauge transformations. When constructing geometric representatives for the $\mu$-map we will need the spaces to be modelled on Banach spaces which admit partitions of unity. We will then require that $p$ is an even integer. 
With this choice of gauge group, possible stabilizers of connections are as follows. The typical connection has stabilizer $\pm 1 \subset Z(\mathrm{SU}(2)) \subset$ $\mathscr{G}$, in which case we call the connection irreducible. If the connection is non-trivial but preserves a splitting $\mathfrak{g}_{E}=\mathbb{R} \oplus L$, where $L$ is a complex line bundle, then the stabilizer of the connection is a circle subgroup $S^{1} \subset \mathscr{G}$. These connections are called reducible. A trivial connection has $\mathrm{SU}(2)$ as its stabilizer. If $H^{1}\left(X ; \mathbb{Z}_{2}\right) \neq 0$ then there is another type of connection with special holonomy group that we need to keep in mind, those which preserve a splitting $\mathfrak{g}_{E}=\lambda \oplus L$ where now $\lambda$ is a non-orientable real line bundle and $L$ is a non-orientable real two plane bundle with orientation bundle isomorphic to $\lambda$. These special (irreducible) connections are called twisted reducible connections.

We let $\mathscr{A}^{*} \subset \mathscr{A}$ denote the space of irreducible connections. The quotient $\mathscr{A}^{*} / \mathscr{G}$ is denoted by $\mathscr{B}^{*}=\mathscr{B}_{\kappa}^{w, *}=\mathscr{B}_{\kappa}^{w, *}(X)$. This quotient space is a Banach manifold. If we fix a conformal structure on $X$ specified by a choice of smooth metric $g$ then we have the moduli space of $g$-ASD connections

$$
M_{\kappa}^{w}=M_{\kappa}^{w}(X)_{g} \subset \mathscr{B}_{\kappa}^{w}(X) .
$$

If we fix a base point $x \in X$ then we can form the space of gauge equivalence classes of framed connections $\mathscr{B}^{o}=\mathscr{B}_{\kappa}^{o, w}=\mathscr{B}_{\kappa}^{o, w}(X, x)$. Let $F_{E} \rightarrow X$ denote the orthogonal frame bundle of $\mathfrak{g}_{E}$. Then $\mathscr{B}^{\circ}$ is the quotient of $\mathscr{A} \times\left(F_{E}\right)_{x}$ by $\mathscr{G}$. It is easy to show that $\mathscr{B}^{\circ}$ is a smooth Banach manifold (for example using the proof of the slice theorem in [29]). The group $\mathrm{SU}(2)$ acts on $\mathscr{B}^{\circ}$ by change of frame with quotient $\mathscr{B}$. Finally let $\mathscr{B}^{o, *}$ denote the quotient $\left(\mathscr{A}^{*} \times\left. F_{E}\right|_{x}\right) / \mathscr{G}$.

The generic metrics theorem of [16], as usually formulated in [16] and [11], requires the 4-manifold $X$ to be simply connected. An examination of the argument, however, shows that this hypothesis is needed only to remove problems which might occur with twisted reducibles. Twisted reducible connections have \pm 1 as their stabilizer with our choice of gauge group, but since they become reducible when restricted to balls in $X$ they require special care. Note that if $\lambda$ is a non-trivial real line bundle with $b^{+}(\lambda)=0$, and if $\mathfrak{g}_{E}$ admits a reduction $\lambda \oplus L$, then the moduli space $M$ will contain a family of twisted reducible connections parametrized by a torus of dimension $b^{1}(\lambda)$. If the formal dimension of $M$ is less than $b^{1}(\lambda)$, then the moduli space must be irregular, and this holds regardless of the choice of metric. When the formal dimension is 
$b^{1}(\lambda)$ or larger, however, the moduli space will be regular for generic metrics. The proof of the statements in the lemma below are only slight modifications of the arguments usually applied to reducible connections [16], [8].

Lemma 2.4. Let $X$ be a 4-manifold, $\lambda$ a non-trivial line bundle, $E$ a 2-plane bundle for which $\mathfrak{g}_{E}$ admits a reduction as $\lambda \oplus L$, and $M_{\kappa}^{w}(X)_{g}$ the moduli space corresponding to a choice of metric $g$. Suppose $c(E)$ is non-zero.

(a) If $b^{+}(\lambda)=0$, then $M_{\kappa}^{w}(X)_{g}$ contains twisted reducibles corresponding to $\lambda$ for all $g$. If the formal dimension of $M_{\kappa}^{w}(X)_{g}$ is at least $b^{1}(\lambda)$, then the twisted reducibles are smooth points of $M_{\kappa}^{w}(X)_{g}$ for generic choice of $g$.

(b) Suppose $b^{+}(\lambda)=1$, and let $g_{t}$ be a 1-parameter family of metrics giving a parametrized moduli space $\mathcal{M}_{\kappa}^{w}$. If the formal dimension of $M_{\kappa}^{w}(X)_{g}$ is at least $b^{1}(\lambda)-1$, then for a generic perturbation of the path $g_{t}$, the parametrized moduli space $\mathcal{M}_{\kappa}^{w}$ will be smooth at the twisted reducibles.

(c) If $b^{+}(\lambda)>1$, then there will be no twisted reducibles corresponding to $\lambda$ in a generic path of metrics.

Corollary 2.5. For a generic metric on $X$, the moduli spaces $M_{\kappa}^{w}$ are smooth manifolds, except at flat or reducible connections. For a generic path of connections, the same is true of the parametrized moduli space.

Proof. We have only to look at non-flat, twisted reducible connections. Suppose $\lambda$ is a non-trivial real line bundle with $b^{+}(\lambda)=0$, and that $\mathfrak{g}_{E}$ has a decomposition as $\lambda \oplus L$. Because $b^{+}(\lambda)-b^{1}(\lambda)+b^{0}(\lambda)$ is independent of $\lambda$, we have

$$
b^{+}(\lambda)-b^{1}(\lambda)=b^{+}-b^{1}+1,
$$

and since $b^{+}(\lambda)=0$, we conclude that $b^{+}-b^{1}+1 \leq 0$. We therefore obtain

$$
\begin{aligned}
b^{1}(\lambda) & =-\left(b^{+}-b^{1}+1\right) \\
& \leq-3\left(b^{+}-b^{1}+1\right) \\
& <8 \kappa-3\left(b^{+}-b^{1}+1\right) .
\end{aligned}
$$

So the previous lemma applies. When we look at a generic path of metrics, we must look also at real line bundles with $b^{+}(\lambda)=1$. Here 
we similarly find $b^{+}-b^{1}+1 \leq 1$. If $b^{+}-b^{1}+1 \leq 0$, then we have

$$
\begin{aligned}
b^{+}(\lambda) & =-\left(b^{+}-b^{1}+1\right)+1 \\
& \leq-3\left(b^{+}-b^{1}+1\right)+1 \\
& <8 \kappa-3\left(b^{+}-b^{1}+1\right)+1,
\end{aligned}
$$

and the previous lemma applies again, because the right-hand side is the formal dimension of the parametrized moduli space. The only remaining case is $b^{+}-b^{1}+1=1$, in which case $b^{1}(\lambda)=0$, and the formal dimension of the parametrized moduli space is $8 \kappa-2$, which is non-negative because $\kappa$ must be at least $\frac{1}{4}$. So again, the lemma gives smoothness. q.e.d.

Remark. Note that this argument makes rather special use of the formula for the formal dimension. Unlike Lemma 2.4, which applies equally well to situations such as manifolds with cylindrical ends, the Corollary is special to closed manifolds. When we consider invariants defined using singular anti-self-dual connections, or moduli spaces associated to cylindrical-end manifolds, we will need to treat twisted reducibles differently.

(ii) Geometric representatives for the $\mu$-map. If $N$ is a smooth manifold (not necessarily compact) and $\mu$ is a rational cohomology class of degree $\delta$ on $N$, then by a geometric representative of $\mu$ we shall mean, in this section, the following data: (i) a closed, smoothly stratified subset $V \subset N$ with a normally oriented top stratum of codimension $\delta$ and all other strata of codimension $\delta+2$ or more; and (ii) a rational coefficient $c$. These should be such that the pairing of $\delta$ with a homology-class $\kappa$ is given by picking a smooth singular cycle representing $\kappa$, in general position with respect to all strata of $V$, and then taking $c$ times the count (with signs) of the intersection points between the cycle and the top stratum of $V$.

Recall that there is a universal $\mathrm{SO}(3)$-bundle

$$
\mathbf{U} \rightarrow \mathscr{B}^{*} \times X,
$$

defined as the quotient $\left(\mathscr{A}^{*} \times F_{E}\right) / \mathscr{G}$, and there is a map $\mu: H_{i}(X ; \mathbb{R}) \rightarrow$ $H^{4-i}\left(\mathscr{B}^{*} ; \mathbb{R}\right)$ given by the slant product $\mu(\alpha)=-\frac{1}{4} p_{1}(\mathbf{U}) / \alpha$. For $\alpha \in H_{2}(X ; \mathbb{Z})$, Donaldson shows in [8] how to construct geometric representatives for $\mu(\alpha)$ which have good properties with respect to the Uhlenbeck compactification of $M_{\kappa}^{w}(X)$. We will now explain how to do the same for $\alpha \in H_{0}(X), H_{1}(X)$ or $H_{3}(X)$. 
Lemma 2.6. Let $V \subset X$ be a closed submanifold (possibly with boundary). Let $\mathscr{B}^{*, *}(X) \subset \mathscr{B}^{*}(X)$ denote those gauge equivalence classes whose restriction to $V$ is irreducible, so there is a restriction map $r: \mathscr{B}^{*, *}(X) \rightarrow \mathscr{B}^{*}(V)$. Then the pull-back $r^{*}\left(\mathscr{B}^{o, *}(V)\right)$ is isomorphic as an $\mathrm{SO}(3)$ bundle to $\mathscr{B}^{o, * * *}(X)$.

Proof. This follows from the existence of a smooth bundle map $\mathscr{B}^{o, *, *}(X) \rightarrow r^{*}\left(\mathscr{B}^{o, *}(V)\right)$ covering the identity. q.e.d.

We will start by constructing representatives for $\mu(x)$ where $x$ is a point of $X$ representing the generator of $H_{0}(X)$. Call a subset $U \subset X$ suitable if:

(i) the map $H_{1}\left(U ; \mathbb{Z}_{2}\right) \rightarrow H_{1}\left(X ; \mathbb{Z}_{2}\right)$ is surjective;

(ii) $U$ is a smooth submanifold with boundary and $U \neq X$.

The construction begins by choosing a suitable closed neighborhood $U$ of $x$. For example, we could take $U$ to be a regular neighborhood of a collection of loops based at $x$ and generating $H_{1}\left(X ; \mathbb{Z}_{2}\right)$.

We digress to explain how to represent the dual cycle to the first Pontryagin class of a 3-plane bundle. Let $M$ be a finite dimensional manifold, not necessarily compact, let $\Xi \rightarrow M$ be a smooth 3-plane bundle and let $\Xi_{\mathbb{C}}$ be its complexification. By definition,

$$
p_{1}(\Xi)=-c_{2}\left(\Xi_{\mathbb{C}}\right) .
$$

The second Chern class of a complex 3-plane bundle is the obstruction to extending a pair of linearly independent sections from the threeskeleton of $X$ to the four-skeleton. The dual class to the second Chern class can be constructed as follows. A pair of sections defines a section of the bundle $\operatorname{Hom}_{\mathbb{C}}\left(\mathbb{C}^{2}, \Xi_{\mathbb{C}}\right)$. Let $\mathcal{S} \subset \operatorname{Hom}_{\mathbb{C}}\left(\mathbb{C}^{2}, \Xi_{\mathbb{C}}\right)$ be the locus in the total space of this bundle where the map is not of maximal rank. The set $\mathcal{S}$ is easily seen to be a real codimension-four stratified subset whose normal bundle at a rank one map $T \in \operatorname{Hom}_{\mathbb{C}}\left(\mathbb{C}^{2},\left(\Xi_{\mathbb{C}}\right)_{x}\right)$ is naturally identified with $N_{T}=\operatorname{Hom}_{\mathbb{C}}\left(\mathbb{C}^{2} / \operatorname{ker}(T), \Xi_{\mathbb{C}_{x}} / \operatorname{Im}(T)\right)$. The only other stratum in $\mathcal{S}$ consist of the rank zero maps and has real codimension twelve. This space is a complex vector space and hence carries a preferred orientation. Call a pair of sections $s_{1}$ and $s_{2}$ of $\Xi_{\mathbb{C}}$ generic if the section $s=\left(s_{1}, s_{2}\right)$ of $\operatorname{Hom}_{\mathbb{C}}\left(\mathbb{C}^{2}, \Xi_{\mathbb{C}}\right)$ is transverse to $\mathcal{S}$. Then we set $V=V\left(s_{1}, s_{2}\right)$ to be the locus where $s_{1}$ and $s_{2}$ are not linearly independent. This is a codimension-four stratified subset of $M$ with normal bundle along the top stratum identified with the normal 
bundle of $\mathcal{S}$ at a rank one map. Giving the normal bundle the opposite orientation, one obtains the desired geometric representative.

Now consider the base-point fibration $\mathscr{B}^{o, w}(U) \rightarrow \mathscr{B}^{*, w}(U)$ for $U$. Let $\Xi \rightarrow \mathscr{B}^{*, w}(U)$ denote the associated real three plane bundle. Since $U$ is suitable, twisted reducible connections remain irreducible when restricted to $U$. It then follows from the unique continuation theorem (see [11], section 4.3.4) that all irreducible, non-flat ASD connections have irreducible restriction to $U$, so there is a well defined restriction $\operatorname{map} r_{U, \kappa}: M_{\kappa}^{w}(X) \rightarrow \mathscr{B}^{*, w}(U)$ for any $\kappa>0$. Following the argument of Donaldson in [8] or [11], we can then find a pair of generic sections of $\Xi$, say $s_{1}$ and $s_{2}$, so that for all $\kappa$, the pair $r_{U, \kappa}^{*}\left(s_{1}\right), r_{U, \kappa}^{*}\left(s_{2}\right)$ is a generic pair of sections of the pull-back bundle. Then we define $V(x)=V\left(x, U, s_{1}, s_{2}\right)$ to be the locus in $M_{\kappa}^{w}(X)$ where $r^{*}\left(s_{1}\right)$ and $r^{*}\left(s_{2}\right)$ are not linearly independent. Our geometric representative for the dual class to $\mu(x)$ is then given by taking $\frac{1}{4}$ the intersection number with 4cycles transverse to $V(x)$. Notice that the construction of $V\left(x, U, s_{1}, s_{2}\right)$ is compatible with Uhlenbeck limits where there is no bubbling in $U$.

Next we construct representatives for $\mu(\gamma)$ where $\gamma$ is a loop in $X$. Fix an oriented embedded loop $\gamma$ and a base point $x$ on $\gamma$. If $f: K \rightarrow \mathscr{B}_{\kappa}^{w, *}(X)$ is a three-dimensional oriented simplicial complex representing an element of $H_{3}\left(\mathcal{B}_{\kappa}^{w, *}(X) ; \mathbb{Z}\right)$, we can evaluate $\langle\mu(\gamma),[K]\rangle$ as follows. The map $f$ induces a universal bundle $\mathbf{U} \rightarrow K \times \gamma$. Let $\hat{\mathbf{U}}$ be the restriction of $\mathbf{U}$ to $K \times\{x\}$. Then the holonomy around $(k, \gamma)$ as $k$ varies through $K$ defines a bundle map $h: \hat{\mathbf{U}} \rightarrow \hat{\mathbf{U}}$, which we view as a section of $h$ of Ad $\hat{\mathbf{U}}=\hat{\mathbf{U}} \times_{\mathrm{Ad}} \mathrm{SO}(3)$. A generic bundle map is transverse to the identity section and hence is the identity above finitely many points in $K$ each of which is in the interior of a three cell of $K$. At each such point $k$, the differential of $h$ identifies the tangent space of $K$ with the vertical tangent space to Ad $\hat{\mathbf{U}}$ at $k$. Since both of these are oriented three-dimensional vector spaces we can attach a $\operatorname{sign} \epsilon(k)$ to each point $k$. It is straightforward to verify that

$$
\begin{aligned}
\sum_{\{k \mid h(k)=1\}} \epsilon(k) & =-\frac{1}{2}\left\langle p_{1}(\mathbf{U}),[K \times \gamma]\right\rangle \\
& =2 \mu(\gamma)([K]) .
\end{aligned}
$$

Now let $U$ be a suitable closed neighborhood of $\gamma$. Consider the following bundle associated to the base-point fibration over $U$ based at 
$x$ by the adjoint action of $\mathrm{SO}(3)$ acting on itself:

$$
\operatorname{Ad} \mathscr{B}^{o, w}(U)=\mathscr{B}^{o, w}(U) \times{ }_{\mathrm{Ad}} \mathrm{SO}(3) \rightarrow \mathscr{B}^{*, w}(U) .
$$

This bundle has a trivial section, the identity section

$$
s_{0}([A])=[A, 1],
$$

and the tangent space to the fibers along $s_{0}$ is naturally an oriented $\mathbb{R}^{3}$-bundle, in fact the adjoint bundle $\operatorname{Ad} \mathscr{B}^{o, w}(U)$ of the base point fibration. The holonomy around the loop $\gamma$ defines another section $s$ of $\operatorname{Ad} \mathscr{B}^{o, w}(U)$

$$
s([A])=\left[A, \operatorname{hol}_{\gamma, x}(A)\right] .
$$

As above, the suitability of $U$ implies that there is a well-defined restriction map $r_{U, \kappa}: M_{\kappa}^{w}(X) \rightarrow \mathscr{B}^{*, w}(U)$ for any $\kappa$. We then find a section $s^{\prime}$ homotopic to $s$ so that for all $\kappa$, the pull-back $r_{U, \kappa}^{*}\left(s^{\prime}\right)$ is transverse to $r_{U, \kappa}^{*}\left(s_{0}\right)$. Then we define $V(\gamma)=V\left(\gamma, U, s^{\prime}\right)$ to be the set of connections where the values of $s_{0}$ and $s^{\prime}$ coincide. Thus $V\left(\gamma, U, s^{\prime}\right)$ is a smooth codimension-three submanifold with normal bundle at $[A]$ identified with the fiber of ad $\mathscr{B}^{o, w}(U)$ at $[A]$. In particular, the normal bundle is oriented. Our geometric representative for the dual class to $\mu(\gamma)$ is given by taking $-\frac{1}{2}$ the oriented intersection number of a 3 cycle with $V(\gamma)$. Again, the definition of $V$ is compatible with taking Uhlenbeck limits where there is no bubbling in $U$.

For classes in $H_{3}(X)$, the construction of a codimension-one representative in the moduli spaces is somewhat simpler than in the other cases, because irreducibility is not an issue which causes concern. An integral 3-dimensional class in $X$ can be represented by an oriented, 3dimensional submanifold (not necessarily connected), $Y \subset X$. Define a map $s: M_{\kappa}^{w} \rightarrow \mathbb{R} / \frac{1}{4} \mathbb{Z}$, for all $\kappa$ simultaneously, by setting

$$
s([A])=\text { C.S. }\left(\left.A\right|_{Y}\right) .
$$

(Here we have normalized the Chern-Simons functional on $\mathrm{SO}(3)$ connections by the convention that ensures that the standard $\mathrm{SU}(2)$ functional is a lift to $\mathbb{R} / \mathbb{Z}$ of the Chern-Simons functional of the associated $\mathrm{SO}(3)$ connection.) Let $s^{\prime}$ be a section which is homotopic to $s$ and has transverse zero set on each $M_{\kappa}^{w}$. For example, we can take $s^{\prime}=s-\theta$, where $\theta$ is any regular value. Let $V(Y)=V\left(Y, s^{\prime}\right)$ be the zero-set of 
$s^{\prime}$, oriented by the standard orientation of $\mathbb{R}$. For our geometric representative of the $\mu$ class, we take $\frac{1}{4}$ the oriented intersection number of $V(Y)$ with 1-dimensional cycles.

The construction of codimension-two representatives for the $\mu$-map on two-dimensional homology classes follows just as in Donaldson [10], with the proviso that a 'suitable' neighborhood of $\Sigma$ is used in the construction and that the mod 2 reduction of $c_{1}(w)$ vanishes under restriction to $U$. Thus associated to any smoothly embedded oriented two-manifold $\Sigma$, a suitable closed neighborhood, $U$, of $\Sigma$ and a choice of generic section $s$ of a complex line bundle over $\mathscr{B}^{*, w}(U)$, we get a codimension-two submanifold $V(\Sigma)=V(\Sigma, U, s)$ of $M_{\kappa}^{w}(X)$ compatible with taking Uhlenbeck limits where there is no bubbling on $U$.

With all this in place we can now give the definition of the Donaldson invariants. As we indicated in the introduction, we will view the Donaldson invariants as a linear function on the algebra

$$
\mathbb{A}(X)=\operatorname{Sym}\left(H_{\text {even }}(X ; \mathbb{R})\right) \otimes \Lambda\left(H_{\text {odd }}(X ; \mathbb{R})\right),
$$

where $\operatorname{deg}(\alpha)=4-i$ if $\alpha \in H_{i}(X)$. Fix a typical monomial generating $\mathbb{A}(X)$, say

$$
z=\beta_{1} \beta_{2} \cdots \beta_{r}
$$

where $\beta_{p} \in H_{i_{p}}(X ; \mathbb{Z})$, so that $z$ is of total degree

$$
\delta=\sum_{1}^{r}\left(4-i_{p}\right)
$$

We stipulate that $\beta_{p}$ should be divisible by 2 in case the dimension $i_{p}$ is 2 . For the moment, we do not allow any $\beta_{p}$ to be 4-dimensional.

Choose smoothly embedded, mutually transverse submanifolds $Y_{p}$ representing these classes. Transversality means that at each point $x_{o}$ in $X$, the sum of the codimensions of the submanifolds which pass through $x_{o}$ is at most 4 . So the zero-dimensional classes are represented by distinct points, disjoint from the other submanifolds, each 1-dimensional class is represented by a loop, disjoint from all submanifolds except at most one 3-dimensional manifold, and so on. Now choose suitable neighbourhoods $U_{p}$ (in the above sense), for each submanifold $Y_{p}$. We can arrange that the intersection pattern of the $U_{p}$ is the same as that of the $Y_{p}$, with one exception: because the suitable neighbourhoods may need to include regular neighbourhoods of 
loops, the neighbourhood of a zero-dimensional class may intersect the neighbourhood of a 3 -dimensional class. We will still have

$$
\sum_{x_{o} \in U_{p}}\left(4-i_{p}\right) \leq 5
$$

for all $x_{o}$ in $X$. We can further arrange that the set of points $x_{0}$ at which equality occurs is a union of disjoint balls. A collection of neighborhoods as above is called a good collection of neighborhoods.

As in [34], we will avoid difficulties which arise from trivial and flat connections by making use of the blow-up, $\hat{X}=X \# \overline{\mathbb{C P}}^{2}$. Let $e$ be a smoothly embedded surface representing the class of the exceptional curve. Take $e$ to be disjoint from any of the previous submanifolds and find a suitable closed neighborhood $U(e)$ so as to extend the good collection. Put $\beta_{0}=[e]$ and $U_{0}=U(e)$, and write

$$
\bar{z}=e z=\beta_{0} \beta_{1} \cdots \beta_{r},
$$

which is a class of degree $\bar{\delta}=\delta+2$.

Now we fix a line-bundle $w$ and define $D_{X}^{w}(z)$. If

$$
\delta \not \equiv-2 w^{2}-3\left(b^{+}-b^{1}+1\right) \quad(\bmod 8),
$$

then $D^{w}(z)=0$. Otherwise find $k \in \frac{1}{4} \mathbb{Z}$ so that $\delta=8 k-3\left(b^{+}-b^{1}+1\right)$ and a rank-2 Hermitian bundle $E \rightarrow \hat{X}$ with

$$
\begin{aligned}
& \operatorname{det}(E)=\bar{w}=w+\text { P.D. }([e]) \\
& \text { and } \quad c(E)=\bar{k}=k+\frac{1}{4} \text {. }
\end{aligned}
$$

Consider a generic metric on $\hat{X}$ so that all the moduli spaces $M_{\bar{k}^{\prime}}^{\bar{w}}(\hat{X})$ with $\bar{k}^{\prime} \leq \bar{k}$ are smooth manifolds of the expected dimension. Note that by construction there are no flat connections amongst these moduli spaces, so they are all smooth manifolds and regular solutions of the equations for generic metrics. Find geometric representatives $V\left(\beta_{p}\right)$ in $M_{\bar{k}^{\prime}}^{\bar{w}}$ using the chosen neighbourhoods $U_{p}$ and the constructions described above. Choose these representatives so that they are transverse in all $M_{\bar{k}^{\prime}}^{\bar{w}}$. Consider the intersection

$$
V(\bar{z})=V\left(\beta_{0}\right) \cap \cdots \cap V\left(\beta_{r}\right) .
$$


A straightforward dimension counting argument shows that $V(\bar{z}) \cap$ $M_{\bar{k}^{\prime}}^{\bar{w}}(\hat{X})$ is empty only if $\bar{k}^{\prime}<\bar{k}$, and that the intersection

$$
V(\bar{z}) \cap M_{\bar{k}}^{\bar{w}}(\hat{X})
$$

is a zero-dimensional submanifold of $M_{\bar{k}}^{\bar{w}}(\hat{X})$ with a normal orientation. Following Donaldson [9], we can use $\bar{w}$ and a homology orientation of $X$ to orient $M_{\bar{k}}^{\bar{w}}(\hat{X})$. We can then attach a sign $\epsilon(v)$ to each $v$ in the above intersection by comparing the normal orientation to $z$ with the orientation of $M_{\bar{k}}^{\bar{w}}(\hat{X})$. Finally, the intersection (2.8) is compact. For if $\left[A_{n}\right]$ is a sequence of connections in (2.8) having $[A]$ as a weak Uhlenbeck limit, and if the points of concentration of curvature are $x_{1}$, $\ldots, x_{j}$, then $[A]$ lies in an intersection of the form

$$
V\left(\bar{z}^{\prime}\right) \cap M_{\bar{k}^{\prime}}^{\bar{w}}(\hat{X}),
$$

where $\bar{k}^{\prime} \leq \bar{k}-j$ and $\operatorname{deg} \bar{z}^{\prime} \geq \bar{\delta}-5 j$ by (2.7). The formal dimension of this intersection is at most $-3 j$, so $j$ must be zero, which shows that the sequence $\left[A_{n}\right]$ was strongly convergent. So we are able to define

$$
D_{X}^{w}(z)=\prod c\left(\beta_{p}\right) \sum_{v} \epsilon(v),
$$

where the sum is taken over the points of (2.8) and the factor at the front is the product of the formal factors involved in the geometric representatives of the $\mu$ classes (so $c\left(\beta_{p}\right)=\frac{1}{4}$ if the dimension is 3 and so on). Note that we omit from our notation the dependence of $D_{X}^{w}$ on a homology orientation. Call any $V(z)$ (or $V(\bar{z})$ ) arising as above a good representative for $z$.

To see that the definition of the invariant is independent of the choice of good representative of the homology classes, and to see also that it is linear, consider writing the last class in the product, $\beta_{r}$, as a sum $\beta_{r}^{\prime}+\beta_{r}^{\prime \prime}$. Put

$$
\begin{aligned}
\bar{z}_{0} & =\beta_{0} \beta_{1} \cdots \beta_{r-1} \\
\bar{z}^{\prime} & =\bar{z}_{0} \beta_{r}^{\prime} \\
\bar{z}^{\prime \prime} & =\bar{z}_{0} \beta_{r}^{\prime \prime} .
\end{aligned}
$$

The case that the dimension $i_{r}$ is 0 does not present an issue, so we take it that $i_{r}$ is 1,2 or 3 . The dimension of the intersection

$$
V_{0}=V\left(\bar{z}_{0}\right) \cap M_{\bar{k}}^{\bar{w}}(\hat{X})
$$


is $\left(4-i_{r}\right)$, and the same dimension counting as before shows that it is compact if $i_{r}=2$ or 3 . In these cases, after weighting by the factors $c\left(\beta_{p}\right)$, the intersection $(2.9)$ represents a closed cycle in $\mathscr{B}_{\kappa}^{w}(X)$, and the invariants $D^{w}(z), D^{w}\left(z^{\prime}\right)$ and $D^{w}\left(z^{\prime \prime}\right)$ are the values of the pairings between this cycle and the classes $\mu\left(\beta_{r}\right)$ etc., so the required linearity

$$
D^{w}(z)=D^{w}\left(z^{\prime}\right)+D^{w}\left(z^{\prime \prime}\right)
$$

is a simple consequence of the linearity of $\mu$. In the case that $i_{r}=1$, the intersection $V_{0}$ is 3-dimensional, and may be non-compact. The only non-compactness which the dimension-count allows is that a sequence of connections $\left[A_{n}\right]$ in $V_{0}$ may converge weakly to a limit $[A]$ of charge $\bar{k}-1$, with curvature concentrating at a point $x_{0}$ where $(2.7)$ is an equality. If we let $Z$ be the complement of the union of balls at which equality is achieved in (2.7), and let $\bar{V}_{0}$ be the closure of $V_{0}$ in the Uhlenbeck compactification, then this means that $\bar{V}_{0}$ parametrizes a smooth family of connections on $Z$, and so defines a closed 3-cycle in $\mathscr{B}_{\kappa}^{w}(Z)$. Furthermore, the cycles $\beta_{r}, \beta_{r}^{\prime}$ and $\beta_{r}^{\prime \prime}$ have representatives in $Z$ which still satisfy the same linear relation, because $H_{1}(Z) \cong H_{1}(X)$. So again, the value of the three invariants is given by pairing the classes $\mu\left(\beta_{r}\right)$ etc. with a 3 -cycle in $\mathscr{B}_{\kappa}^{w}(Z)$ and the linearity of $D^{w}$ follows from the linearity of the $\mu$ map.

The fact that $D_{X}^{w}$ is independent of the choice of Riemannian metric is an entirely standard argument, which follows [10].

Remarks. If there are no 3-dimensional classes among the $\beta_{p}$, then the right-hand side of the inequality in (2.7) can be improved to 4 . In this case, in the argument of the last paragraph, $V_{0}$ is compact even when $i_{r}=1$ and the argument is therefore a little simpler.

In the discussion above, we paid no attention to the 4-dimensional class $[X] \in H_{4}(X)$. Since $\mu([X])=\kappa \in H^{0}\left(M_{\kappa}^{w}\right)$, we can incorporate this class by declaring that

$$
D^{w}([X] z)=\kappa D^{w}(z)
$$

for $z \in \mathbb{A}_{\delta}(X)$, where $\delta=8 \kappa-3\left(b^{+}-b^{1}+1\right)$.

Finally, note that the condition that $b^{+}-b^{1}$ is odd is not needed in the definitions we have made: the invariants are potentially non-trivial when $b^{+}-b^{1}$ is even, as long as $b^{1}$ is non-zero. We make this restriction because our attention is soon to be focused on the case $b^{1}(X)=0$. 
(iii) Flat connections on non-trivial circle bundles over a Riemann surface. Let $Y=Y(n, g)$ denote an oriented three-manifold which is the total space of a non-trivial principal $S^{1}$-bundle

$$
p: Y \rightarrow \Sigma
$$

of degree $n$ over a Riemann surface of genus $g$. We orient $Y$ with the opposite orientation to that which it obtains as the boundary of the oriented disk bundle in which the zero-section has self-intersection number $n$. (Thus, if $Y$ arises as the boundary of the complement $X \backslash N$ of the neighbourhood $N$ of a surface $\Sigma$ of self-intersection $n$, then $Y$ is oriented as the boundary of $X \backslash N$.) Although it is not essential, we assume the genus $g$ is at least 2 .

Let $\eta$ denote an $S^{1}$-invariant one-form dual to the generator of the circle action. If we have a metric $h$ on $\Sigma$, we will choose $\eta$ so that $d \eta=-2 n \pi p^{*}(* 1) / V$, where $V$ is the volume of $\Sigma$. On $Y$ we take the metric

$$
h_{Y}=\eta^{2}+p^{*} h .
$$

Let $\mathscr{R}_{+}(\Sigma)$ and $\mathscr{R}_{-}(\Sigma)$ denote the spaces of flat connections modulo gauge equivalence in $\mathfrak{g}_{F}$, where $F$ is a Hermitian 2-plane bundle with even (respectively odd) first Chern class. These are often called the $\mathrm{SU}(2)$ and the $\mathrm{SO}(3)$ representation varieties. In both cases, the gauge group is the determinant one gauge group. Let $c$ be the generator of the center of $\pi_{1}(Y)$, so that $\int_{c} \eta=2 \pi$.

Let $w \rightarrow \Sigma$ be a line bundle, and use the same letter to denote its pull-back to $Y$. Let $F$ be a Hermitian rank-2 bundle on $\Sigma$, with an isomorphism $\operatorname{det} F \rightarrow w$. Let $E$ be the pull-back of $E$ to $Y$, equipped with the same isomorphism $\operatorname{det} E \rightarrow w$. Write $\ell=w \cdot[\Sigma]$. The space of flat connections in $\mathfrak{g}_{E}$ modulo determinant one gauge transformations on $Y$ is denoted $\mathscr{R}^{w}(Y)$. Since $w$ is pulled back from $\Sigma$, the restriction of $\mathfrak{g}_{E}$ to the $S^{1}$ fibres has a preferred lift to $\mathrm{SU}(2)$, so the holonomy of a connection along $c$ has a well-defined interpretation as an element of the special unitary group of the fibre at the base-point.

The space $\mathscr{R}^{w}(Y)$ is a union of connected components

$$
\mathscr{R}^{w}(Y)=\mathscr{R}_{\mathrm{ev}}^{w} \cup \bigcup_{\substack{m \in \mathbb{Z}+\ell / 2 \\ 0<m<n / 2}} \mathscr{R}_{m}^{w} \cup \mathscr{R}_{\mathrm{od}}^{w} .
$$


These components are distinguished the conjugacy class of the image of $c$ under the holonomy representation. In $\mathscr{R}_{\mathrm{ev}}^{w}, c$ is sent to $I$. In $\mathscr{R}_{\mathrm{od}}^{w}, c$ is sent to $-I$. In $\mathscr{R}_{m}^{w}$ all the representations are reducible and $F=L_{m_{1}} \oplus L_{m_{2}}$ where $\ell=m_{1}+m_{2}$ and $m=\frac{1}{2}\left(m_{1}-m_{2}\right)$. With respect to this splitting, $c$ is sent to

$$
\left(\begin{array}{cc}
\exp -2 \pi i m / n & 0 \\
0 & \exp 2 \pi i m / n
\end{array}\right) .
$$

The component $\mathscr{R}_{\mathrm{ev}}^{w}$ is identified with $\mathscr{R}_{+}(\Sigma)$ if $\ell$ is even and with $\mathscr{R}_{-}(\Sigma)$ if $\ell$ is odd. Each connection in $\mathscr{R}_{\mathrm{ev}}^{w}$ is gauge equivalent to one pulled back from $\Sigma$, by a gauge transformation which respects the determinant. The component $\mathscr{R}_{\mathrm{od}}^{w}$ is identified with the moduli space of projectively flat connections on $\Sigma$ with diagonal curvature

$$
\left(\begin{array}{cc}
\pi i(n+\ell) *_{\Sigma} 1 & 0 \\
0 & \pi i(n+\ell) *_{\Sigma} 1
\end{array}\right)
$$

in a bundle $F^{\prime}$ with $c_{1}\left(F^{\prime}\right)=n+\ell$. On the level of connections, the identification is given as follows. First note that $p^{*}(F)=p^{*}\left(F^{\prime}\right)$, and if $\Gamma^{\prime}$ is a connection on $F^{\prime} \rightarrow \Sigma$ with the given curvature, the connection

$$
\Gamma=p^{*}\left(\Gamma^{\prime}\right)+\frac{1}{2}\left(\begin{array}{cc}
i & 0 \\
0 & -i
\end{array}\right) \eta
$$

is the desired flat connection on $p^{*}\left(F^{\prime}\right) \rightarrow Y$. If $n+\ell$ is even, $\mathscr{R}_{\mathrm{od}}^{w}$ is identified with $\mathscr{R}_{+}(\Sigma)$ while if $n+\ell$ is odd $\mathscr{R}_{\text {od }}^{w}$ is identified with $\mathscr{R}_{-}(\Sigma)$. The representation space $\mathscr{R}_{+}(\Sigma)$ is a singular algebraic variety. Thus $\mathscr{R}_{\mathrm{ev}}^{w}$ and $\mathscr{R}_{\mathrm{od}}^{w}$ may also be singular. If so, we denote the set of smooth points of these spaces $\mathscr{R}_{\mathrm{ev} / \mathrm{od}}^{w, s}$ and the set of singular points $\mathscr{R}_{\mathrm{ev} / \mathrm{od}}^{w, s i n g}$. The latter coincides with the locus of reducible connections within these components.

The components $\mathscr{R}_{m}^{w}$ are all diffeomorphic to the Jacobian torus of $\Sigma$. On the level of connections the identification is given as follows. Write $F=L_{m_{1}} \oplus L_{m_{2}}$ as above, and fix a connection $\Gamma_{0}$ in $L_{m_{1}} \otimes$ $L_{m_{2}}=\operatorname{det}(F)$. Consider all connections $\Gamma_{1}, \Gamma_{2}$ in $L_{m_{1}}$ and $L_{m_{2}}$ so that $F_{\Gamma_{i}}=-2 \pi i m_{i} *_{\Sigma} 1$ and $\Gamma_{1} \otimes \Gamma_{2}=\Gamma_{0}$. Then

$$
\Gamma=\pi^{*}\left(\begin{array}{cc}
\Gamma_{1} & 0 \\
0 & \Gamma_{2}
\end{array}\right)+\frac{m}{n}\left(\begin{array}{cc}
i & 0 \\
0 & -i
\end{array}\right) \eta
$$


is the desired projectively flat connection on $E \rightarrow Y$.

The Chern-Simons invariants for the components of $\mathscr{R}^{w}(Y)$ are as follows. In accordance with our convention on the orientation of $Y$, the Chern-Simons invariant is defined by the condition

$$
\text { C. S. }([A]) \equiv-c(\bar{A}) \quad(\bmod 1)
$$

whenever $A$ is a connection in $g_{E} \rightarrow Y$, and $\bar{A}$ is an extension of $A$ to a connection in $\mathfrak{g}_{\bar{E}}$, where $\bar{E}$ is a 2-plane bundle over the disk bundle of degree $n$ with determinant equal to the pull-back of $w \rightarrow \Sigma$. Here $c(A)$ is the Chern-Weil integral (2.3). Note that the characterization of $\bar{E}$ depends on the fact that $\operatorname{det} E$ is given as pulled back from $\Sigma$, and our choice of gauge group ensures that C.S. is well-defined modulo 1.

Proposition 2.11. Let $[\rho] \in \mathscr{R}^{w}(Y)$ be a flat connection. Then the Chern-Simons invariant of $[\rho]$ is
(a) $0 \quad(\bmod 1)$
if $[\rho] \in \mathscr{R}_{\mathrm{ev}}^{w}$,
(b) $-n / 4(\bmod 1)$
if $[\rho] \in \mathscr{R}_{\mathrm{od}}^{w}$,
(c) $m^{2} / n(\bmod 1)$
if $[\rho] \in \mathscr{R}_{m}^{w}$.

(iv) The moduli space on cylindrical end manifolds. In this section we review the results from [36],[41],[40] on the moduli space for cylindrical end manifolds. Recall that a manifold with cylindrical end modeled on $Y$ is a smooth manifold together with the following data:

(i) a compact submanifold, $K=K(Z) \subset Z$;

(ii) a diffeomorphism $Z \backslash \operatorname{int}(K) \rightarrow \mathbb{R}_{+} \times Y$. If $Z$ is oriented we require this diffeomorphism to be orientation preserving.

A cylindrical end bundle $E \rightarrow Z$ over a cylindrical end manifold is a smooth bundle together with a bundle isomorphism $\left.E\right|_{Z \backslash \operatorname{Int}(K)} \rightarrow$ $\mathbb{R}_{+} \times E_{Y}$ for some bundle $E_{Y} \rightarrow Y$. We will suppress the urge to give these identifications explicitly. Always in this paper, $Y$ will be a circle bundle over a Riemann surface $\Sigma$, equipped with a Riemannian metric $h_{Y}$ as in equation (2.10).

Let $Z$ denote an oriented Riemannian 4-manifold with cylindrical end modeled on $Y$. Fix a cylindrical-end Hermitian 2-plane bundle $E$ over $Z$ and an isomorphism $\psi: \operatorname{det} E \rightarrow w$, where $w$ is a cylindrical-end line bundle and $\delta$ is constant on the end of $Z$. The moduli space of finite energy ASD projective connections of charge $\kappa$, denoted $M_{\kappa}^{w}(Z)$, is the space of smooth connections $A$ on $\mathfrak{g}_{E}$ satisfying the conditions

$$
F_{A}=-* F_{A}
$$




$$
c(A)=\kappa
$$

modulo the group of smooth, determinant-one gauge transformations of $E$. The moduli space $M_{\kappa}^{w}(Z)$ is given the quotient topology when both the space of projective connections and gauge group are given the topology of $C^{\infty}$-uniform convergence on compact sets of $Z$.

According to [36] and [41], there is a continuous map $r: M_{\kappa}^{w}(Z) \rightarrow$ $\mathscr{R}^{w}(Y)$ given as follows. For each gauge equivalence class of ASD connections, $[A]$, the limit

$$
r([A])=\lim _{t \rightarrow \infty}\left[\left.A\right|_{\{t\} \times Y}\right]
$$

exists and is a gauge equivalence class of flat connections on $Y$. Furthermore, for all $[A] \in M_{k}(Z)$ we have the equality

$$
\kappa \equiv \text { C.S. }(r([A])) \quad(\bmod 1) .
$$

If $S \subset \mathscr{R}^{w}(Y)$, we set $M_{\kappa}^{w}(Z ; S)=r^{-1}(S)$. If $Z$ has more than one end there is boundary map corresponding to each end. We will encounter the two-ended manifold $\mathbb{R} \times Y$ and will write $r_{+}$for the limit as $t \rightarrow+\infty$ and $r_{-}$for the limit as $t \rightarrow-\infty$.

As discussed in [36] Chapter V, the map $r$ has a locally defined refinement that will be important for us.We review a bit of material from [36] for the reader's convenience and refer the reader there for more details. For a flat connection $\Gamma$ on $E \rightarrow Y$, let $S_{\Gamma}$ denote a slice through $\Gamma$ for the action of the gauge group on connections on $E$. Then an ASD connection on $\mathbb{R} \times E$ with sufficiently small action is gauge equivalent to a connection $A=\Gamma+a(t)+\alpha(t) d t$ for a path of one-forms $a(t)$ in $S_{\Gamma}$ and $\alpha(t)$ a path of zero-forms orthogonal to $\operatorname{Ker}\left(\Delta_{\Gamma}\right)$. This gauge transformation is unique up to the action of the stabilizer of $\Gamma$. The path of one-forms $a(t)$ satisfies the gradient flow equation for the Chern-Simons function on the slice. This gradient flow equation can be shown to have a center manifold, i.e. a finite dimensional submanifold tangent to the vector field and tangent at $\Gamma$ to $H^{1}(Y ; \operatorname{ad} \Gamma)$. The main result that we will need is the following.

Lemma 2.12. With $A$ as above there is a unique decomposition

$$
A=\Gamma+\gamma_{A}(t)+b(t)
$$

where $\gamma_{A}(t)$ is a path of one-forms corresponding to a flow in a center manifold and $b(t)$ is an exponentially decaying path of one-forms. 
In order to state the results on the formal dimension of the moduli spaces it is convenient to consider the compactification $\hat{Z}$ of $Z$ which adds a copy of $\Sigma$ as the zero section of the punctured disk bundle $\mathbb{R}_{+} \times Y$. To fix our orientations, let $Z$ have a cylindrical end modelled on $Y(n, g)$. The self-intersection of the surface $\Sigma$ in $\hat{Z}$ is then $n$. We shall always suppose that $E_{Y}$ and its determinant $w$ are identified with bundles pulled back from $\Sigma$; as in the previous subsection, this extra data allows us unambiguously to label the components of $\mathscr{R}^{w}(Y)$ as $\mathscr{R}_{\mathrm{ev}}^{w}$ etc. (and at the same time it determines a distinguished extension of $E$ to $\hat{Z}$ ). According to [36] and [41] there are deformation complexes for the part of the moduli spaces which lies over the smooth points. In the case where $n>0$ there is also a deformation complex for the part of the moduli space lying over the singular strata of $\mathscr{R}_{\mathrm{ev} / \mathrm{od}}^{w}$. The indices of these complexes are given in the following theorem, as well as the appropriate version of the generic metrics theorem.

Theorem 2.13. If $n>0$ then:

$\left(a_{+}\right) \quad$ The formal dimension of $M_{\kappa}^{w}\left(Z ; \mathscr{R}_{\mathrm{ev} / \mathrm{od}}^{w, s}\right)$ is

$$
8 \kappa-3\left(b^{+}(\hat{Z})-b^{1}(\hat{Z})+1\right)
$$

and the formal dimension of $M_{\kappa}^{w}\left(Z ; \mathscr{R}_{\mathrm{ev}}^{w, s i n g}\right)$ and $M_{\kappa}^{w}\left(Z ; \mathscr{R}_{\mathrm{od}}^{w, s i n g}\right)$ is

$$
8 \kappa-3\left(b^{+}(\hat{Z})-b^{1}(\hat{Z})+1\right)-4 g+5 .
$$

$\left(b_{+}\right) \quad$ The formal dimension of $M_{\kappa}^{w}\left(Z ; \mathscr{R}_{m}^{w}\right)$ is

$$
8 \kappa-3\left(b^{+}(\hat{Z})-b^{1}(\hat{Z})+1\right)-8 \frac{m^{2}}{n}+4 m-2 g+3 .
$$

If $n<0$, then:

$\left(a_{-}\right) \quad$ The formal dimension of $M_{\kappa}^{w}\left(Z ; \mathscr{R}_{\mathrm{ev} / \mathrm{od}}^{w, s}\right)$ is

$$
8 \kappa-3\left(b^{+}(\hat{Z})-b^{1}(\hat{Z})+1\right) .
$$

$\left(b_{-}\right) \quad$ The formal dimension of $M_{\kappa}^{w}\left(Z ; \mathscr{R}_{m}^{w}\right)$ is

$$
8 \kappa-3\left(b^{+}(\hat{Z})-b^{1}(\hat{Z})+1\right)-8 \frac{m^{2}}{n}+4 m-2 g+2 .
$$

Furthermore if $\kappa>0$ then for a generic cylindrical end metric on $Z$ the moduli spaces $M_{\kappa}^{w}\left(Z ; \mathscr{R}_{\mathrm{ev} / \mathrm{od}}^{w, s}\right)$ and $M_{\kappa}^{w}\left(Z ; \mathscr{R}_{m}^{w}\right)$ are smooth manifolds having the dimension given by the above formulae, except possibly at reducible or twisted reducible connections. The same applies 
to $M_{\kappa}^{w}\left(Z ; \mathscr{R}_{\mathrm{ev} / \mathrm{od}}^{w, s i n g}\right)$ in the case $n>0$, except that this is a smoothly stratified singular space, with top stratum of the given dimension. If $b^{+}(Z)>0$ then there are no reducible connections in these moduli spaces for generic metric. If $b^{+}(Z)>1$ then there are no reducible connections for a generic one-parameter family of metrics.

Recall from [36] that on cylindrical end manifold, by $b^{ \pm}(Z)$ we mean the dimension of a positive (respectively negative) dimensional subspace for the quadratic form on

$$
\operatorname{Im}\left(H_{c}^{2}(Z ; \mathbb{R}) \rightarrow H^{2}(Z ; \mathbb{R})\right),
$$

where $H_{c}^{*}$ denotes the cohomology with compact supports. We make a similar definition if the coefficients of the cohomology groups are in a non-trivial real line bundle $\lambda$.

Definition 2.14. A cylindrical-end 4-manifold $Z$ is called admissible if $\hat{Z}$ is admissible in the sense of definition 2.1 and for every nontrivial real line bundle $\lambda \rightarrow Z$ which is trivial on the end of $Z$ we have $b^{+}(\lambda)>1$.

With this definition twisted reducible connections do not present a problem, as the following lemma shows.

Lemma 2.15. Let $Z$ be an admissible cylindrical-end 4-manifold. Then the conclusion of Theorem 2.13 holds without mention of twisted reducible connections.

Proof. We must show that for a generic metric (and a generic path of metrics) all twisted reducible connections are smooth points of their moduli spaces. From the definition of admissible it is immediate that for generic paths of metrics there are no twisted reducible connections for which the corresponding real line bundle is trivial on the end of $Z$. Now consider a twisted reducible ASD-connection $A$ whose corresponding line bundle is non-trivial on the end of $Z$. The boundary value of $A$ is necessarily in the smooth irreducible part of the representation variety, $\mathscr{R}_{\mathrm{ev} / \mathrm{od}}^{w, s}(Y)$. For this part of the moduli space the dimension formulae are exactly the same as for closed manifold $\hat{Z}$ and hence the argument of Corollary 2.5 applies to complete the proof. q.e.d.

There are similar statements for the cases $g=0$ and $g=1$, the difference being that some parts of the representation varieties are absent in these cases.

(v) Singular connections. We will draw on the results laid out in [26] and [27], with some minor adaptations which are made necessary 
by our framework, which allows $E$ to have non-trivial determinant. We summarize some of the main points here. See also [25].

Let $(X, \Sigma)$ be a pair consisting of an admissible 4-manifold and an oriented, connected, smoothly-embedded 2-manifold. As before, let $E$ be a Hermitian 2-plane bundle on $X$ with a fixed isomorphism $\psi$ : $\operatorname{det}(E) \rightarrow w$. Let $N$ be a tubular neighbourhood of $\Sigma$, and on $N$ let there be given a reduction of $E$ as an (ordered) sum of line bundles: $\left.E\right|_{N}=L_{1} \oplus L_{2}$. The associated bundle $\mathfrak{g}_{E}$ then has a reduction to $\mathrm{SO}(2)$, so $\mathfrak{g}_{E}=\mathbb{R} \oplus K$. We orient $K$ by identifying it with the complex line bundle $L_{1} \otimes L_{2}^{-1}$. Note that there is no determinant-one gauge transformation which interchanges $L_{1}$ and $L_{2}$, and therefore no gauge transformation which changes the orientation of $K$. We record the characteristic numbers

$$
\begin{aligned}
\kappa & =c(E) \in \frac{1}{4} \mathbb{Z} \\
l & =-\frac{1}{2} e(K)[\Sigma] \in \frac{1}{2} \mathbb{Z} .
\end{aligned}
$$

We call these the instanton number and the monopole number respectively. (The asymmetry between the roles the of $L_{1}$ and $L_{2}$ is apparent in the correspondence theorem (see section 6 and [27], [5]) which identifies the moduli spaces of singular connections with moduli spaces of stable bundles with parabolic structure in the case that $(X, \Sigma)$ is complex; in this case, $L_{1}$ corresponds to a distinguished holomorphic line-subbundle of the restriction of $E$ to $\Sigma$.)

Fix a Riemannian metric on $X$. Then we have moduli spaces

$$
M_{\kappa, l}^{w, \alpha}
$$

of ASD connections in $\left.\mathfrak{g}_{E}\right|_{X \backslash \Sigma}$ with prescribed holonomy at $\Sigma$. The holonomy around a small, positively-oriented loop linking $\Sigma$ is an anticlockwise rotation of the $K$ plane through angle $4 \pi \alpha$. We take $\alpha$ in the open interval $\left(0, \frac{1}{2}\right)$. The gauge group again consists of determinant-one gauge transformations. Other details of the definition and construction of $M^{\alpha}$ can be found in [26]. We only recall the following facts.

Lemma 2.16. For $A \in M_{\kappa, l}^{w, \alpha}$, the Chern-Weil integral $c(A)$ is given by

$$
c(A)=\kappa+2 \alpha l-\alpha^{2} Q(\Sigma) .
$$


Lemma 2.17. The formal dimension of the moduli space $M_{\kappa, l}^{w, \alpha}$ is given by

$$
\begin{aligned}
\operatorname{dim} M_{\kappa, l}^{w, \alpha} & =\delta \\
& =8 \kappa+4 l-3\left(b^{+}-b^{1}+1\right)-2(g-1) .
\end{aligned}
$$

For a generic choice of Riemannian metric, $M_{\kappa, l}^{w, \alpha}$ is a smooth manifold of dimension $\delta$, except possibly at flat, reducible and twisted reducible connections. There is also a space $M_{\kappa, l}^{w, *}$ with a map to the interval $\left(0, \frac{1}{2}\right)$ whose fibres are the moduli spaces $M_{\kappa, l}^{w, \alpha}$. For generic metric, $M_{\kappa, l}^{w, *}$ is a smooth $(\delta+1)$-manifold, except possibly at flat, reducible and twisted reducible connections.

As in [27] and [25], we shall not, in fact, use a smooth Riemannian metric on $X$ when exploiting these moduli spaces: we shall use a metric which has a cone-like (orbifold) singularity in the normal directions along $\Sigma$, with cone-angle $2 \pi / \nu$, where $\nu$ is an integer chosen so that $2 \pi / \nu$ is small compared to both $\alpha$ and $\frac{1}{2}-\alpha$. The reason for this is that it allows one to sharpen the weak compactness theorem for sequences of connections in the moduli spaces: see section 8 of [26].

It is often convenient to introduce the integer

$$
p=(g-1)-2 l
$$

in place of the half-integer $l$. We then label the moduli spaces with the decoration $p$ as a superscript, rather than the subscript $l$, thus $M_{\kappa}^{w, p, \alpha}$. The dimension formula then reads

$$
\delta=8 k-3\left(b^{+}-b^{1}+1\right)-2 p
$$

or

$$
\operatorname{dim} M_{\kappa}^{w, p, \alpha}=\operatorname{dim} M_{\kappa}^{w}-2 p
$$

Notice that $p$ must satisfy the parity condition

$$
p \equiv(g-1)-w \cdot[\Sigma](\bmod 2)
$$

We now use these moduli spaces, as in [27] and [25] to define invariants of pairs $(X, \Sigma)$. Although it is possible to construct these as 
linear functions on $\mathbb{A}(X)$ (see [25]), we content ourselves with defining invariants which are linear functions

$$
D_{X, \Sigma}^{w, p}: \mathbb{A}(X / \Sigma) \rightarrow \mathbb{R}
$$

Since $X$ may not be simply connected, we need to deal first with potential problems with twisted reducibles.

Observe that if there is a non-flat connection in the moduli space, compatible with a reduction $\mathfrak{g}_{E}=\lambda \oplus L$, then $L$ must coincide with $\pm K$ in the neighbourhood $N$ of $\Sigma$, and therefore $\lambda$ must be trivial on $\Sigma$. (So if $H_{1}(\Sigma ; \mathbb{Z} / 2)$ maps onto $H_{1}(X ; \mathbb{Z} / 2)$, then $\lambda$ must be trivial on all of $X$.) With this understood, we note (without further proof):

Lemma 2.22. The statement of Lemma 2.4 continues to hold with $M_{\kappa}^{w, p, \alpha}$ in place of $M_{\kappa}^{w}$, with the proviso that only reductions $\lambda \oplus L$ with $\left.L\right|_{\Sigma}= \pm K$ are considered.

Note that Corollary 2.5 does not extend to the moduli spaces of singular connections; the problem comes when $p$ is large, so that the dimension formula is substantially different. We therefore make the following definition:

Definition 2.23. The pair $(X, \Sigma)$ is admissible if $X$ is admissible in the sense of Definition 2.1 and $b^{+}(\lambda)>1$ for all real line bundles $\lambda$ whose restriction to $\Sigma$ is trivial.

If $X$ is admissible, then a sufficient condition for $(X, \Sigma)$ to be admissible is that $b^{+}(X)-b^{1}(X) \geq 1$, for then $b^{+}(\lambda)>2$ for all non-trivial $\lambda$. Another sufficient condition, as noted above, is that $H_{1}(\Sigma ; \mathbb{Z} / 2)$ maps onto $H_{1}(X ; \mathbb{Z} / 2)$.

With this in place, we can define $D_{X, \Sigma}^{w, p}$ provided the pair is admissible and $p$ satisfies the parity condition (2.20). Just as we defined $D_{X}^{w}$. We form the connected sum $\bar{X}=X \# \overline{\mathbb{C P}}^{2}$ at a point well away from $\Sigma$. Let

$$
z=\beta_{1} \beta_{2} \cdots \beta_{r}
$$

be a monomial in $\mathbb{A}(X / \Sigma)$ of degree $\delta$. If

$$
\delta \not \equiv-2 w^{2}-3\left(b^{+}-b^{1}+1\right)-2 p \quad(\bmod 8)
$$

then $D^{w, p}(z)=0$. Otherwise, let $M_{\kappa}^{w, p, \alpha}(X, \Sigma)$ be the moduli space whose formal dimension is $\delta$. Put $\beta_{0}=e \in H_{2}\left(\overline{\mathbb{C P}}^{2}\right)$ and represent the classes $\beta_{0}, \ldots \beta_{r}$ by submanifolds of $\bar{X} \backslash S$. Take a good collection of 
neighbourhoods, and then a good representative

$$
V(\bar{z})=V\left(\beta_{0}\right) \cap \cdots \cap V\left(\beta_{r}\right),
$$

so that the intersection

$$
V(\bar{z}) \cap M_{\bar{\kappa}}^{\bar{w}, p, \alpha}(\bar{X}, \Sigma)
$$

is a compact zero-manifold. Here $\bar{\kappa}=\kappa+\frac{1}{4}$ and $\bar{w}=w+e$ as before. Using the standard orientation of the moduli space (depending on a homology-orientation of $X$ ), one orients the points of the zero-manifold and defines $D^{w, p}(z)$ as before. See [25] for more details. The value of $D^{w, p}(z)$ is independent of $\alpha$ (on account of $b^{+}(X)$ being bigger than 1 ).

Finally, we will also have use for moduli spaces in the mixed case where we have $Z$ with a cylindrical end and $\Sigma^{\prime} \subset Z$ a compact, oriented, embedded Riemann surface. We wish to study finite energy ASD connections on $Z$ singular along $\Sigma^{\prime}$ with holonomy parameter $\alpha$. Our data then includes the topological class, $l$, of the reduction along $\Sigma^{\prime}$, as above. We denote the corresponding moduli space

$$
M_{\kappa, l}^{w, \alpha}\left(Z, \Sigma^{\prime}\right)
$$

where now $\kappa$ is defined by the following equation:

$$
c(A)=\kappa+2 \alpha l-\alpha^{2} Q\left(\Sigma^{\prime}\right) .
$$

With this convention we still have

$$
\kappa \equiv \text { C.S. }(r([A])) \quad(\bmod 1) .
$$

There are similar dimension formulae and generic metrics results in this case.

3. Tunneling between flat connections on circle bundles

(i) Statement of results. Let $Y$ be a circle bundle of degree $n$ over a Riemann surface $\Sigma$ of genus $g$. We assume that $n$ is positive and that $g$ is at least 2 . We equip $Y$ with a standard Riemannian metric as in (2.10), and we orient it as $Y(n, g)$. Let $w$ be a line bundle on $Y$ pulled back from $\Sigma$, and recall the corresponding representation variety 
$\mathscr{R}^{w}(Y)$. We usually use $\rho$ and $\sigma$ to denote typical flat connections on $Y$. Define a relation on $\mathscr{R}^{w}(Y)$ as follows:

Definition 3.1. The flat connection $\rho$ is $\kappa$-related to $\sigma$, written $\rho \bumpeq{ }_{\kappa} \sigma$, if there is an anti-self-dual connection $A$ on $\mathbb{R} \times Y$ whose ChernWeil integral $c(A)$ is not more than $\kappa$, with end-limits $\rho=r_{-}(A)$ and $\sigma=r_{+}(A)$.

In this definition, the maps $r_{+}$and $r_{-}$are the end-limit maps for connections on the cylinder, as discussed section 2(iv). Our aim here is to address some issues of dimension and transversality which are concerned not with the moduli spaces $M_{\kappa}^{w}(\mathbb{R} \times Y)$ themselves, but with the relations $\bumpeq_{k}$ which result. For any subset $K$ in $\mathscr{R}^{w}(Y)$, we define

$$
\begin{aligned}
& \vec{T}_{\kappa}(K)=\left\{\rho \mid \exists x \in K \text { with } x \bumpeq_{\kappa} \rho\right\} \\
& \overleftarrow{T}_{\kappa}(K)=\left\{\rho \mid \exists x \in K \text { with } \rho \bumpeq_{\kappa} x\right\}
\end{aligned}
$$

Let us say that a subset $K$ of a manifold $Z$ is contained in dimension $\delta$ if it is contained in the image of a finite collection of smooth maps from manifolds of dimension at most $\delta$.

Theorem 3.2. For each integer $k$ with $0 \leq k \leq n$, there is a smooth manifold $U_{k}$ of dimension $6 g-6+6 k$ and a map

$$
t=\left(t_{1}, t_{2}\right): U_{k} \rightarrow \mathscr{R}_{\mathrm{ev}}^{w, s} \times \mathscr{R}_{\mathrm{ev}}^{w, s}
$$

with the property that if $\rho, \sigma$ are elements of $\mathscr{R}_{\mathrm{ev}}^{w, s}$ and either $\rho \bumpeq_{k} \sigma$ or $\sigma \bumpeq_{k} \rho$, then $(\rho, \sigma) \in t\left(U_{k}\right)$. Furthermore, $t_{2}$ is a submersion. There is a similar statement for $\mathscr{R}_{\mathrm{od}}^{w, s}$.

Corollary 3.3. If $K \subset \mathscr{R}_{\mathrm{ev}}^{w, s}$ is contained in dimension $\delta$ and $k \leq n$, then $\vec{T}_{k}(K) \cap \mathscr{R}_{\mathrm{ev}}^{w, s}$ and $\overleftarrow{T}_{k}(K) \cap \mathscr{R}_{\mathrm{ev}}^{w, s}$ are both contained in dimension $\delta+6 k$. The same statement is true with $\mathscr{R}_{\mathrm{od}}^{w, s}$ replacing $\mathscr{R}_{\mathrm{ev}}^{w, s}$.

The above Corollary is the prototype for the results of this section. Although it deals with the case that will eventually be most important, we will have need also of information about the relation $\bumpeq_{\kappa}$ in connection with other pairs of components of $\mathscr{R}^{w}(Y)$. First we deal with trajectories between $\mathscr{R}_{\mathrm{od}}^{w}$ and $\mathscr{R}_{\mathrm{ev}}^{w}$.

Proposition 3.4. If $\rho \in \mathscr{R}_{\mathrm{ev}}^{w}$ and $\sigma \in \mathscr{R}_{\mathrm{od}}^{w}$, then we cannot have $\rho \bumpeq{ }_{\kappa} \sigma$ or $\sigma \bumpeq_{\kappa} \rho$ for any $\kappa<n / 4$.

Next, we consider the singular strata in $\mathscr{R}_{\mathrm{ev} / \mathrm{od}}^{w, \text { sing }}$. These are the sets of 
reducible connections, which may be empty:

$$
\mathscr{R}_{\mathrm{ev} / \mathrm{od}}^{w, \operatorname{sing}}=\mathscr{R}_{\mathrm{ev} / \mathrm{od}}^{w} \backslash \mathscr{R}_{\mathrm{ev} / \mathrm{od}}^{w, s}
$$

Proposition 3.5. Let $K \subset \mathscr{R}_{\mathrm{ev}}^{w, s i n g}$ be contained in dimension $\delta$. If $1 \leq k<n$, then $\overleftarrow{T}_{k}(K) \cap \mathscr{R}_{\mathrm{ev}}^{w, s}$ is contained in dimension $\delta-2+6 k$ The same holds with od replacing ev.

Last in this sequence is a proposition which deals similarly with the components $\mathscr{R}_{m}^{w} \subset \mathscr{R}^{w}(Y)$ consisting entirely of reducible connections.

Proposition 3.6. Let $K \subset \mathscr{R}_{m}^{w}(0<m<n / 2)$ be contained in dimension $\delta$.

(a) If $\kappa<n(1-m / n)^{2}$, then $\overleftarrow{T}_{\kappa}(K) \cap \mathscr{R}_{\mathrm{ev}}^{w, s}$ is contained in dimension

$$
\delta+2 g-4+4 m+6\left(\kappa-m^{2} / n\right)
$$

and is empty if $\kappa<m^{2} / n$.

(b) If $\kappa<\left(n-m^{\prime} / n\right)^{2}$, then $\overleftarrow{T}_{\kappa}(K) \cap \mathscr{R}_{\mathrm{od}}^{w, s}$ is contained in dimension

$$
\delta+2 g-4+4 m^{\prime}+6\left(\kappa-\left(m^{\prime}\right)^{2} / n\right)
$$

where $m^{\prime}=(n / 2)-m$, and is empty if $\kappa<\left(m^{\prime}\right)^{2} / n$.

The formulae in Corollary 3.3 and the last two Propositions can be best understood by considering the dimensions of the corresponding anti-self-dual moduli spaces on the cylinder. To illustrate this, consider first the moduli space $M=M_{k}^{w}\left(\mathbb{R} \times Y ; \mathscr{R}_{\mathrm{ev}}^{w, s}, K\right)$ for some $\delta$-dimensional submanifold $K \subset \mathscr{R}_{\mathrm{ev}}^{w, s}$. We use the notation

$$
M_{\kappa}^{w}\left(\mathbb{R} \times Y ; S_{1}, S_{2}\right)=\left(r_{-}\right)^{-1}\left(S_{1}\right) \cap\left(r_{+}\right)^{-1}\left(S_{2}\right)
$$

for subsets $S_{1}, S_{2}$ in $\mathscr{R}^{w}(Y)$. The formal dimension of $M$ is $\delta+8 k$. Because of the action of translations on $\mathbb{R} \times Y$, one expects $r_{-}(M) \subset \overleftarrow{T}_{k}$ $(K) \cap \mathscr{R}_{\mathrm{ev}}^{w, s}$ to have dimension at most $\delta+8 k-1$. In fact, because $Y$ has a circle action, and because the induced circle action on $\mathscr{R}^{w}(Y)$ is trivial, one expects a further collapsing, so that $r_{-}(M)$ should have dimension at most $\delta+8 k-2$. Corollary 3.3 confirms this naive dimension count in the case $k=1$. For larger $k$, it gives a stronger result, though $\delta+8 k-2$ would have sufficed instead of $\delta+6 k$ for our later application.

The other two propositions have a similar interpretation: in each case, the dimension of the relevant part of $\overleftarrow{T}_{\kappa}(K)$ has dimension at 
least two less than the formal dimension of a corresponding moduli space on the cylinder.

There are other cases to consider for tunneling between the various components of $\mathscr{R}^{w}(Y)$. The statements above are all that are needed for our present purposes, but we record one other which we can prove with the same techniques. This is the case of tunneling between two of the components $\mathscr{R}_{m}^{w}$. The proof of this one will be omitted.

Proposition 3.7. Let $m_{+}$and $m_{-}$be integers in the range $0<$ $m<n / 2$ and let $K \subset \mathscr{R}_{m_{+}}^{w}$ be contained in dimension $\delta$. Suppose $\kappa<n+\left(m_{+}^{2}-m_{-}^{2}\right) / n$. Then $\overleftarrow{T}_{\kappa}(K) \cap \mathscr{R}_{m_{-}}^{w}$ is contained in dimension

$$
\delta+4\left(m_{+}-m_{-}\right)+6\left(\kappa-\left(m_{+}^{2}-m_{-}^{2}\right) / n\right)-2
$$

if $m_{-}<m_{+}+\kappa-\left(m_{+}^{2}-m_{-}^{2}\right) / n$; it is contained in dimension

$$
\delta+4\left(m_{+}-m_{-}\right)+6\left(\kappa-\left(m_{+}^{2}-m_{-}^{2}\right) / n\right)
$$

if $m_{-}=m_{+}+\kappa-\left(m_{+}^{2}-m_{-}^{2}\right) / n$, and is empty if $m_{-}>m_{+}+\kappa-\left(m_{+}^{2}-\right.$ $\left.m_{-}^{2}\right) / n$.

There is a category of cases not covered by these propositions, namely the sets of the form $\stackrel{\leftarrow}{T}(K) \cap \mathscr{R}_{m}^{w}$ for $K \subset \mathscr{R}_{\mathrm{ev} / \mathrm{od}}^{w}$. A statement closely analogous to those above would not be true in these remaining cases, and it seems likely that obtaining the best possible result here would require more delicate arguments, involving the stratification of $\mathscr{R}_{\mathrm{ev} / \mathrm{od}}^{w}$ which appears in section 6 .

(ii) A result of Guo. Let us equip the line bundle $w \rightarrow \Sigma$ with a holomorphic structure and denote the holomorphic line bundle by $\omega$. We can then regard the elements of $\mathscr{R}^{w}(Y)$ as connections in $E$ such that the induced connection in $\operatorname{det} E$ is the pull-back of the unique $U(1)$ connection in $w$ compatible with the holomorphic structure. Associated with any $\rho$ and $\sigma$ are stable or semi-stable holomorphic bundles $\mathscr{E}_{\rho}$ and $\mathscr{E}_{\sigma}$ with determinant $\omega$. Our immediate aim is a result which gives a necessary condition for $\rho$ to be $\kappa$-related to $\sigma$ in terms of complex geometry. Let $W$ be the ruled surface obtained by closing up $\mathbb{R} \times Y$ by adding the surfaces $\Sigma_{+}$and $\Sigma_{-}$, with the convention that $Q\left(\Sigma_{+}\right)=$ $n>0$. This $W$ has a complex structure which is compatible, on the complement of the two surfaces $\Sigma_{ \pm}$, with the Riemannian metric on the cylinder. Note that the ruling provides a canonical identification of both $\Sigma_{+}$and $\Sigma_{-}$with $\Sigma$. Let $F$ be a fiber of the ruling, and let $\omega$ 
denote also the pull-back of the line bundle from $\Sigma$ to $W$. Note that $\omega^{2}$ is zero on $W$.

According to Guo [20], when the metric $h_{Y}$ on $Y$ is of the form (2.10), the product metric $d t^{2}+h_{Y}$ on $\mathbb{R} \times Y$ is conformal to a Kähler metric $\sigma^{2}\left(d t^{2}+h_{Y}\right) ;$ the conformal factor is

$$
\sigma^{2}=\exp \left(\frac{2 \pi n}{V} t\right) .
$$

For such metrics, Guo proves a correspondence theorem giving a setwise identification between, on the one hand, the subspace of the antiself-dual moduli space on the cylinder consisting of connections whose end limits are smooth points of the representation variety, and on the other hand a moduli space of holomorphic bundles on the ruled surface. For our purposes we find it useful to extend Guo's result to cover the singular part of the representation variety, in the direction of obtaining holomorphic data on the ruled surface from an anti-self-dual connection on the cylinder. In this section we record the statements of this extension of Guo's results, and then we reprove Guo's results, assuming a technical result of his, from a somewhat different point of view which allows us to take care of the singular part of the representation variety. We begin with the simplest case to state, where the linking holonomy is trivial.

Proposition 3.8. To each $[A] \in M_{\kappa}^{w}\left(\mathbb{R} \times Y ; \mathscr{R}_{\mathrm{ev}}^{w}, \mathscr{R}_{\mathrm{ev}}^{w}\right)$ we can associate a rank-2 holomorphic bundle $\mathscr{E}_{A}=\mathscr{E} \rightarrow W$ with determinant $\omega$ and $c_{2}(\mathscr{E})=\kappa$, such that the restriction of $\mathscr{E}_{A}$ to $W \backslash\left\{\Sigma_{+} \cup \Sigma_{-}\right\}$ is equivalent to the holomorphic structure induced by the anti-self-dual connection A. Furthermore the holomorphic bundle $\left.\mathscr{E}\right|_{\Sigma_{+}}$is isomorphic to the poly-stable bundle induced by the flat connection $r_{+}(A)$, while the holomorphic bundle $\left.\mathscr{E}\right|_{\Sigma_{-}}$is semi-stable and s-equivalent to the polystable bundle induced by $r_{-}(A)$.

(A rank-2 bundle is poly-stable if it is either stable or a sum of two line-bundles of the same degree. Every semi-stable bundle has a filtration for which the associated graded bundle is poly-stable. Following [39], two semi-stable bundles are s-equivalent if their associated graded bundles are the same).

The case of central linking holonomy is similar. Recall that there is a natural identification of $\mathscr{R}_{\text {od }}^{w}$ with the moduli space of rank-2 holomorphic vector bundles $\mathscr{F}$ with $\operatorname{det}(\mathscr{F})=\omega+\Lambda$, where $\Lambda$ is any 
holomorphic line bundle with $\operatorname{deg}(\Lambda) \equiv n \bmod 2$. Thus a rank-2 holomorphic bundle $\mathscr{E} \rightarrow W$ with $\left.\operatorname{det}(\mathscr{E})\right|_{\Sigma_{+}}=\left.\omega\left[\Sigma_{+}\right]\right|_{\Sigma_{+}}$defines an element of $\mathscr{R}_{\mathrm{od}}^{w}\left(\Sigma_{+}\right)$provided that $\left.\mathscr{E}\right|_{\Sigma_{+}}$is semistable. A similar statement holds for $\left[\Sigma_{-}\right]$. We again use $c(\mathscr{E})$ to denote the characteristic number $c_{2}(\mathscr{E})-\frac{1}{4} c_{1}^{2}(\mathscr{E})$.

Proposition 3.9. To each $[A] \in M_{\kappa}^{w}\left(\mathbb{R} \times Y ; \mathscr{R}_{\mathrm{ev} / \mathrm{od}}^{w}, \mathscr{R}_{\mathrm{ev} / \mathrm{od}}^{w}\right)$ we can associate a rank-2 holomorphic bundle $\mathscr{E}_{A}=\mathscr{E} \rightarrow W$ with c $(\mathscr{E})=$ $\kappa$ such that the restriction of $\mathscr{E}_{A}$ to $W \backslash\left\{\Sigma_{+} \cup \Sigma_{-}\right\}$is equivalent to the holomorphic structure induced by the anti-self-dual connection $A$. If $r_{-}(A) \in \mathscr{R}_{\mathrm{ev}}^{w}$ and $r_{+}(A) \in \mathscr{R}_{\mathrm{od}}^{w}$, then we can take $\operatorname{det}(\mathscr{E})=\omega+\left[\Sigma_{+}\right]$. If $r_{-}(A) \in \mathscr{R}_{\mathrm{od}}^{w}$ and $r_{+}(A) \in \mathscr{R}_{\mathrm{ev}}^{w}$, then we can take $\operatorname{det}(\mathscr{E})=\omega+\left[\Sigma_{-}\right]$. If $r_{+}(A)$ and $r_{-}(A)$ are both in $\mathscr{R}_{\mathrm{od}}^{w}$, then we can take $\operatorname{det}(\mathscr{E})=\omega+$ $\left[\Sigma_{-}+\Sigma_{+}\right]$. In all three cases the holomorphic bundle $\left.\mathscr{E}\right|_{\Sigma_{+}}$is isomorphic to the poly-stable bundle corresponding to the flat connection $r_{+}(A)$, while the holomorphic bundle $\left.\mathscr{E}\right|_{\Sigma_{-}}$is semi-stable and s-equivalent to the poly-stable bundle corresponding to $r_{-}(A)$.

Next we consider the case where the linking holonomy at $+\infty$ is non-central. For a line bundle $\mathscr{L} \rightarrow \Sigma$, we introduce the normalized degree

$$
\operatorname{deg}_{\omega} \mathscr{L}=\operatorname{deg} \mathscr{L}-\frac{1}{2} \operatorname{deg} \omega .
$$

Recall that $\mathscr{R}_{m}^{w}$ can be canonically identified with $J_{m}$, the Jacobian torus of line bundles with $\operatorname{deg}_{\omega}=-m$.

Proposition 3.10. To each $[A] \in M_{\kappa}^{w}\left(\mathbb{R} \times Y ; \mathscr{R}_{\mathrm{ev} / \mathrm{od}}^{w}, \mathscr{R}_{m}^{w}\right)$, we can associate a pair $\left(\mathscr{E}_{A}, \mathscr{L}_{A}\right)=(\mathscr{E}, \mathscr{L})$, where $\mathscr{E} \rightarrow W$ is a rank-2 holomorphic bundle and $\mathscr{L} \rightarrow \Sigma_{+}$is a holomorphic line subbundle, $\left.\mathscr{L} \hookrightarrow \mathscr{E}\right|_{\Sigma_{+}}$. We have $c(\mathscr{E})=\kappa-m^{2} / n$, the restriction of $\mathscr{E}_{A}$ to $W \backslash\left\{\Sigma_{+} \cup \Sigma_{-}\right\}$is isomorphic to the holomorphic structure induced by the anti-self-dual connection $A$, and $\operatorname{deg}_{\omega}(\mathscr{L})=-m$. If $r_{-}(A) \in \mathscr{R}_{\mathrm{ev}}^{w}$ we can take $\mathscr{E}$ to have determinant $\omega$. If $r_{-}(A) \in \mathscr{R}_{\mathrm{od}}^{w}$ we can take $\mathscr{E}$ with $\operatorname{det}(\mathscr{E})=\omega+\left[\Sigma_{-}\right]$. The holomorphic bundle $\left.\mathscr{E}\right|_{\Sigma_{-}}$is semi-stable and $s$-equivalent to the bundle induced by the flat connection $r_{-}([A])$. The point in $J_{m}$ determined by $\mathscr{L}$ corresponds to $r_{+}([A])$.

There is a similar statement for $M_{\kappa}^{w}\left(\mathbb{R} \times Y ; \mathscr{R}_{m}^{w}, \mathscr{R}_{\mathrm{ev} / \mathrm{od}}^{w}\right)$ where $c(\mathscr{E})=$ $\kappa+m^{2} / n$, the degree of the line bundle $\mathscr{L}$ is $m$ and $s$-equivalence is replaced by isomorphism. Finally, we have:

Proposition 3.11. To each $[A] \in M_{\kappa}^{w}\left(\mathbb{R} \times Y ; \mathscr{R}_{m_{-}}^{w}, \mathscr{R}_{m_{+}}^{w}\right)$, we can associate a triple $\left(\mathscr{E}_{A}, \mathscr{L}_{-, A}, \mathscr{L}_{+, A}\right)=\left(\mathscr{E}, \mathscr{L}_{-}, \mathscr{L}_{+}\right)$, where $\mathscr{E} \rightarrow W$ is a 
holomorphic line bundle with determinant $\omega$ and $\mathscr{L}_{ \pm} \rightarrow \Sigma_{ \pm}$are holomorphic line subbundles $\left.\mathscr{L}_{ \pm} \rightarrow \mathscr{E}\right|_{\Sigma_{ \pm}}$. We have

$$
c(\mathscr{E})=\kappa+\left(m_{-}^{2}-m_{+}^{2}\right) / n
$$

and the restriction of $\mathscr{E}_{A}$ to $W \backslash\left\{\Sigma_{+} \cup \Sigma_{-}\right\}$is isomorphic to the holomorphic structure induced by the anti-self-dual connection $A$. We have $\operatorname{deg}_{\omega}\left(\mathscr{L}_{+}\right)=-m_{+}$, and $\operatorname{deg}_{\omega}\left(\mathscr{L}_{-}\right)=m_{-}$, and the points in $J_{m_{ \pm}}$determined by these line bundles correspond to $r_{+}(A)$ and $r_{-}(A)$.

The remainder of this subsection is devoted to the proof of these results. Let $N_{ \pm}$denote complex tubular neighborhood of $\Sigma_{ \pm}$in the ruled surface $W$. Let $\pi: N_{ \pm} \rightarrow \Sigma_{ \pm}$denote the bundle projection. Also let $N_{ \pm}^{o}=N_{ \pm} \backslash \Sigma_{ \pm}$. Let $r$ denote the distance from $\Sigma_{ \pm}$in $N_{ \pm}^{o}$, let $\eta_{ \pm}$ denote a connection one-form on $N_{ \pm}^{o}$, and let $v$ denote the dual vector field to $\eta$ which generates the circle action. Under the natural identification of $N_{+}$with $N_{-}$we have $\eta_{+}=-\eta_{-}$. Let $E \rightarrow N_{ \pm}$be a unitary 2-plane bundle pulled back from $E^{\Sigma} \rightarrow \Sigma_{ \pm}$. A choice of connection $A$ on $\left.E\right|_{N^{\circ}}$ with $F_{A}^{0,2}=0$ determines a holomorphic structure $\mathcal{E}_{A}$ on $\left.E\right|_{N_{ \pm}^{o}}$. Guo's theorem gives conditions under which the holomorphic structure extends over $N_{ \pm}$in terms of properties of the connection.

Theorem 3.12. Let $A$ be a connection on $\left.E\right|_{N^{\circ}}$ with $F_{A}^{0,2}=0$. Suppose that there is a connection $B$ on $E^{\Sigma}$ and constants $\delta, M>0$ so that

$$
\begin{aligned}
A & =\pi^{*}(B)+a \\
& =\pi^{*}(B)+a_{r} d r+a_{\eta} r \eta+b
\end{aligned}
$$

where $b$ is a one-form which annihilates the tangent spaces to the fibres of the projection to $\Sigma$, and the forms $a_{r}, a_{\eta}$ and $b$ satisfy the pointwise estimates

$$
\begin{array}{ll} 
& r^{-\delta}|b|+r^{1-\delta}\left(\left|a_{r}\right|+\left|a_{\eta}\right|\right) \leq M \\
& r^{-\delta}\left|\nabla_{\frac{\partial}{\partial u}} b\right|+r^{1-\delta}\left(\left|\nabla_{\frac{1}{r} v} b\right|+\left|\nabla_{\frac{\partial}{\partial r}} b\right|\right) \leq M \\
\text { and } \quad & r^{2-\delta}\left(\left|\nabla_{\frac{1}{r} v} a_{r}\right|+\left|\nabla_{\frac{1}{r} v} a_{\eta}\right|+\left|\nabla_{\frac{\partial}{\partial r}} a_{r}\right|+\left|\nabla_{\frac{\partial}{\partial r}} a_{\eta}\right|\right) \leq M,
\end{array}
$$

where $\nabla$ denotes the covariant derivative of $\pi^{*}(B)$ and $\frac{\partial}{\partial u}$ is any unit tangent vector to $N_{ \pm}^{o}$ in the horizontal space for $\eta_{ \pm}$. Then there is a holomorphic structure $\hat{\mathcal{E}}_{\hat{A}}$ on the bundle $E \rightarrow N_{ \pm}$, induced by a connection $\hat{A}$, and a continuous complex gauge transformation $h: E \rightarrow E$ so that $\left(\left.h\right|_{N^{\circ}}\right)^{*}\left(\bar{\partial}_{A}\right)=\left.\bar{\partial}_{\hat{A}}\right|_{N^{\circ}}$. Furthermore:

(i) If $\hat{\mathcal{E}}$ and $\hat{\mathcal{E}}^{\prime}$ are two such holomorphic structures in $E \rightarrow N$, 
then there is a continuous gauge transformation $h$ of $E$ such that $h^{*}\left(\bar{\partial}_{\hat{A}^{\prime}}\right)=\bar{\partial}_{\hat{A}}$.

(ii) The restriction of the holomorphic structure to $\Sigma,\left.\hat{\mathcal{E}}_{\hat{A}}\right|_{\Sigma}$, is equivalent to that induced by $B$.

(iii) Finally, if we choose $\hat{A}$ (as we may) so that it agrees with $A$ outside a smaller neighbourhood of $\Sigma$, then $\int_{N^{\circ}} \operatorname{tr}\left(F_{A} \wedge F_{A}\right)=$ $\int_{N} \operatorname{tr}\left(F_{\hat{A}} \wedge F_{\hat{A}}\right)$.

The essence of Guo's proof is, following an argument due to Bando [4], that these conditions imply that, after passing to a high order local branched cover, the connection is in $L_{1}^{p}$ for some $p>2$. Then Guo can use Buchdahl's holomorphic version of Uhlenbeck's basic gauge fixing lemma [7] to define the holomorphic structure. If we identify the cylinder $\mathbb{R}_{ \pm} \times Y$ with $N_{ \pm}^{o}$ by the map $(t, y) \mapsto\left(e^{\mp} t, y\right)$, these condition translate directly into the connection having $C^{1}$ exponential decay to $\pi^{*}(B)$.

In [36] it was shown that any finite energy ASD connection on $[0, \infty)$ $\times Y$ could be uniquely decomposed as an exponentially small perturbation of a connection corresponding to a flow in the center manifold. If $A$ is a finite energy ASD connection on $[0, \infty) \times( \pm Y)$ with nonexponential decay, then its limit $r([A])$ is a reducible flat connection in $\mathscr{R}^{w}(Y)$. As in section 2(iii), we can choose a representative $\Gamma$ for $r([A])$ which is $S^{1}$-invariant.

Lemma 3.13. For an $S^{1}$-invariant metric on $Y$, there is a center manifold through $\Gamma$ consisting of $S^{1}$-invariant connections.

Proof. For an $S^{1}$-invariant metric on $Y$, the Hodge representatives for the cohomology groups for $\Gamma$ are also $S^{1}$ invariant. As observed in [36], the center manifold can be chosen to be set-wise invariant under any compact group action preserving the vector field. The center manifold is then given as the graph of a smooth equivariant function $h: U \subset H^{1}(Y ; \operatorname{ad} \Gamma) \rightarrow \operatorname{Ker}\left(d_{\Gamma}^{*}\right)$. The result now follows from the $S^{1}$-invariance of Hodge representatives of the cohomology. q.e.d.

To make use of this result we study the ASD equation on $S^{1}$-invariant connections. An $S^{1}$-invariant connection $A$ on $\mathfrak{g}_{E} \rightarrow Y$ is of the form $A=B+\Phi \eta+\Psi \mathrm{dt}$ where the zero-forms $\Phi$ and $\Psi$ and the connection $B$ are pulled back from $\mathfrak{g}_{E^{\Sigma}}$. The ASD equation for $S^{1}$-invariant 
connections on $\mathbb{R} \times Y$ then becomes the pair of equations

$$
\begin{aligned}
\frac{\partial B}{\partial t} & =-*_{\Sigma} d_{B} \Phi+d_{B} \Psi \\
& =\partial_{B} \overline{(\Psi+i \Phi)}+\bar{\partial}_{B}(\Psi+i \Phi) \\
\frac{\partial \Phi}{\partial t} & =\frac{-n}{V} \Phi-*_{\Sigma} F_{B}+\frac{1}{2}[\Phi, \Psi]
\end{aligned}
$$

where the volume of $\Sigma$ is $2 \pi V$.

Lemma 3.15. Let $A(t)=B(t)+\Phi(t) \eta+\Psi(t) d t$ be a smooth, $S^{1}$-invariant $A S D$ connection on $\left(T_{0}, T_{1}\right) \times \mathfrak{g}_{E}$ (the $T_{i}$ maybe infinite). Define a complex gauge transformation of $\left(T_{0}, T_{1}\right) \times \mathfrak{g}_{E}$ as the (smooth) solution to the $O D E$

$$
\frac{\partial g}{\partial t}=-(\Psi+i \Phi) g
$$

with the initial condition say $g(0)=I$. Then

$$
g \cdot A=B(0) .
$$

Proof. The action of $\mathcal{G}_{\mathbb{C}}$ on the space of connections is given by the formula

$$
g \cdot A=A+g^{-1} \bar{\partial}_{A} g-\left(g^{-1} \bar{\partial}_{A} g\right)^{*} .
$$

First we check that this gauge transformation takes the path of connections $B(t)$ to the constant connection $B(0)$. Consider the time derivative

$$
\begin{aligned}
\frac{\partial}{\partial t}\left(g^{-1} \bar{\partial}_{B} g\right)= & -g^{-1} \dot{g} g^{-1} \bar{\partial}_{B} g+g^{-1}\left[\Pi^{0,1} \dot{B}, g\right]+g^{-1} \bar{\partial}_{B} \dot{g} \\
& =g^{-1}((\Psi+i \Phi) g) g^{-1} \bar{\partial}_{B} g+g^{-1}\left[\bar{\partial}_{B}(\Psi+i \Phi), g\right] \\
& +g^{-1} \bar{\partial}_{B}(-(\Psi+i \Phi) g) \\
& =-\bar{\partial}_{B}(\Psi+i \Phi) .
\end{aligned}
$$

From this we calculate the time derivative of $g \cdot B$ :

$$
\begin{aligned}
\frac{\partial g \cdot B}{\partial t} & =\frac{\partial B}{\partial t}+\frac{\partial}{\partial t}\left(g^{-1} \bar{\partial}_{B} g-\left(g^{-1} \bar{\partial}_{B} g\right)^{*}\right) \\
& =0
\end{aligned}
$$


from (3.14). Hence $g \cdot B=B(0)$. Next we determine the action of this gauge transformation on the connection $A=B+\Phi \eta+\Psi d t$. We have

$$
\begin{aligned}
g^{-1} \bar{\partial}_{B+\Phi \eta+\Psi d t} g & =g^{-1} \Pi^{0,1}\left(d_{B+\Phi \eta+\Psi d t} g\right) \\
& =g^{-1} \Pi^{0,1}\left(d t \dot{g}+d_{B}^{\Sigma} g+\eta[\Phi, g]+d t[\Psi, g]\right) \\
& =\frac{1}{2}((-\Psi+i \Phi) d t+(i \Psi-\Phi) \eta)+g^{-1}\left(\bar{\partial}_{B}^{\Sigma} g\right)
\end{aligned}
$$

Hence

$$
\begin{aligned}
g \cdot A= & B+\Phi \eta+\Psi d t+\frac{1}{2}((-\Psi+i \Phi) d t+(i \Psi-\Phi) \eta)+g^{-1}\left(\bar{\partial}_{B}^{\Sigma} g\right) \\
& -\frac{1}{2}((-\Psi+i \Phi) d t+(i \Psi-\Phi) \eta)^{*}+g^{-1}\left(\bar{\partial}_{B}^{\Sigma} g\right)^{*} \\
= & g \cdot B+\Phi \eta+\Psi d t+\frac{1}{2}((-\Psi+i \Phi) d t+(i \Psi-\Phi) \eta) \\
& -\frac{1}{2}((\Psi+i \Phi) d t+(i \Psi+\Phi) \eta) \\
= & B(0) . \quad \text { q.e.d. }
\end{aligned}
$$

To analyze the case of boundary values in $\mathscr{R}_{m}^{w}$ we will need more detailed information about the asymptotics of the connection. At a flat connection $\Gamma$ on $Y$, let $S_{\Gamma}=\left\{a \in \Omega^{1}\left(Y ; \mathfrak{g}_{E}\right) \mid d_{\Gamma}^{*} a=0\right\}$ denote the slice for the gauge group action on connections on $Y$, then the Hessian of Chern-Simons Hess $\Gamma_{\Gamma}$ CS is the operator

$$
-* d_{\Gamma}: S_{\Gamma} \rightarrow S_{\Gamma}
$$

This is an essentially self-adjoint operator with discrete real spectrum unbounded in both directions. If $\Gamma$ is a reducible connection preserving the splitting $\mathfrak{g}_{E}=\mathbb{R} \oplus L$, then there is a corresponding decomposition of the slice $S_{\Gamma}=S_{\Gamma}(\mathbb{R}) \oplus S_{\Gamma}(L)$. We can assume that $L$ is pulled back from a bundle $L_{\ell} \rightarrow \Sigma$ with connection, where $c_{1}\left(L_{\ell}\right)=\ell$. Let $\mathcal{L} \rightarrow \Sigma$ denote the corresponding holomorphic line bundle. By tensoring with the normal bundle of $\Sigma$, we can also assume that $0<|\ell| \leq n / 2$ (that is we take $\ell=2 m$ if $0<m \leq \frac{n}{4}$ and $\ell=2 m-n$ if $\frac{n}{4} \leq m<\frac{n}{2}$ ).

Proposition 3.16. Let $\Gamma$ be a flat connection representing a point of $\mathscr{R}_{m}^{w}(Y)$. Then the spectrum of $\mathrm{Hess}_{\Gamma}$ CS has the following properties.

(i) The kernel of $\left.\operatorname{Hess}_{\Gamma} \mathrm{CS}\right|_{S_{\Gamma}(\mathbb{R})}$ is identified with $H^{1}(Y$; ad $\Gamma)$.

(ii) The smallest positive and negative eigenvalues of $\left.\mathrm{Hess}_{\Gamma} \mathrm{CS}\right|_{S_{\Gamma}\left(L_{m}\right)}$ are $\pm \frac{\ell}{n}$. The eigenspace corresponding to $\frac{\ell}{n}$ is identified with the pull-back from $\Sigma$ of the harmonic representatives of the Dolbeault 
cohomology group $H^{1,0}(\Sigma ; \mathcal{L})$ while the eigenspace corresponding to $-\frac{\ell}{n}$ is similarly identified with $H^{0,1}(\Sigma ; \mathcal{L})$.

Proof. Item $(i)$ is a standard fact. Let $P_{\ell} \rightarrow \Sigma$ be the total space of a principal $S^{1}$-bundle with Euler class $\ell$ and let $L_{\ell}$ denote the associated complex line bundle. The pull-back $\pi^{*}\left(P_{\ell}\right)$ is the Whitney product bundle of $Y \rightarrow \Sigma$ and $P_{\ell} \rightarrow \Sigma$. This makes $\pi^{*}\left(P_{\ell}\right)$ into the total space of a principal $S^{1} \times S^{1}$ bundle over $\Sigma$, where the first $S^{1}$ is the circle action coming from $P_{\ell}$ and the second comes from $Y$. The second $S^{1}$-action decomposes the space of sections of $\pi^{*}\left(L_{\ell}\right)$ into irreducible representations of $S^{1}$. We say that a section has weight $k$ if it transforms according to $f\left(p \cdot\left(1, e^{i \theta}\right)\right)=e^{-i k \theta} f(p)$. A section of weight $k$ then transforms under the diagonal action as $f\left(p \cdot\left(e^{i \theta}, e^{i \theta}\right)\right)=e^{-i(1-k) \theta} f(p)$, and as such defines a section of $N^{\otimes k} \otimes L_{\ell} \rightarrow \Sigma$, where $N$ is the C-bundle associated to $Y$. The generator, $v$, of the circle action on $Y$ acts on sections of weight $k$ by the formula $\nabla_{v}^{\Gamma} f=-i k f$.

Now suppose that $b+\varphi \eta$ is an eigenvector for $-* d_{\Gamma}$ with eigenvalue $\lambda$ where $b$ and $\varphi$ have weight $k$. We have

$$
\begin{aligned}
-* d_{\Gamma}(b+\varphi \eta) & \\
& =-*\left(\eta \wedge \nabla_{v}^{\Gamma} b+d_{\Gamma^{\prime}}^{\Sigma} b+i \frac{\ell}{n} \eta \wedge b+\frac{n}{V} *^{\Sigma} 1 \varphi+d_{\Gamma^{\prime}}^{\Sigma} \varphi \wedge \eta\right) \\
& =-*\left(-i k \eta \wedge b+d_{\Gamma^{\prime}}^{\Sigma} b+i \frac{\ell}{n} \eta \wedge b+\frac{n}{V} *^{\Sigma} 1 \varphi+d_{\Gamma^{\prime}}^{\Sigma} \varphi \wedge \eta\right) \\
& =i\left(k-\frac{\ell}{n}\right) *^{\Sigma} b+*^{\Sigma} d_{\Gamma^{\prime}}^{\Sigma} \varphi+\eta \wedge\left(\frac{-n}{V} \varphi-* d_{\Gamma^{\prime}}^{\Sigma} b\right),
\end{aligned}
$$

where we consider $\varphi$ as a section of $N^{\otimes k} \otimes L_{\ell}$ and $b$ as a section of $N^{\otimes k} \otimes L_{\ell} \otimes_{\mathbb{R}} T^{*} \Sigma$ and $\Gamma^{\prime}$ is the connection in $N^{\otimes k} \otimes L_{\ell}$ induced by the connection in $L$ and $i \eta$ in $N$. Thus the condition that $b+\varphi \eta$ is an eigenvector gives

$$
-* d_{\Gamma^{\prime}}^{\Sigma} b=\left(\lambda+\frac{n}{V}\right) \varphi \quad \text { and } \quad *^{\Sigma} d_{\Gamma^{\prime}}^{\Sigma} \varphi=\left(\lambda-i\left(k-\frac{\ell}{n}\right) *^{\Sigma} b\right) .
$$

The slice condition now reads.

$$
\begin{aligned}
d_{\Gamma}^{*}(b+\varphi \eta) & =-*\left(\eta \wedge d_{\Gamma^{\prime}} *^{\Sigma} b+d_{\Gamma^{\prime}} *^{\Sigma} \varphi+\eta \wedge \nabla^{\Gamma^{\prime}} *^{\Sigma} \varphi+i \frac{\ell}{n} \eta \wedge *^{\Sigma} \varphi\right) \\
& =d_{\Gamma^{\prime}}^{*} b+i\left(k-\frac{\ell}{n}\right) \varphi \\
& =0 .
\end{aligned}
$$


The argument now breaks up into two case according to whether or not $\varphi=0$.

If $\varphi=0$ then using the decomposition

$$
N^{\otimes k} \otimes L_{\ell} \otimes_{\mathbb{R}} T^{*} \Sigma=N^{\otimes k} \otimes L_{\ell} \otimes_{\mathbb{C}} T^{1,0} \Sigma \oplus N^{\otimes k} \otimes L_{\ell} \otimes_{\mathbb{C}} T^{0,1} \Sigma,
$$

we see that if $b$ is a section of $N^{\otimes k} \otimes L_{\ell} \otimes_{\mathbb{C}} T^{1,0} \Sigma$ (respectively $N^{\otimes k} \otimes$ $\left.L_{\ell} \otimes_{\mathbb{C}} T^{0,1} \Sigma\right)$ with $d_{\Gamma} b=0$, then $d_{\Gamma}^{*} b=0$ and $(b, 0)$ is an eigenvector with eigenvalue $k-\ell / n$ (respectively $k+\ell / n$ ) and by the choice of $\ell$ the result holds in this case.

Suppose that $\varphi \neq 0$. Decompose $b$ as $b^{\prime}+b^{\prime \prime}$, as above. Let

$$
\partial_{\Gamma^{\prime}} \partial_{\Gamma^{\prime}}^{*}: \Omega^{0,1}\left(\Sigma ; N^{\otimes k} \otimes L_{\ell}\right) \rightarrow \Omega^{0,1}\left(\Sigma ; N^{\otimes k} \otimes L_{\ell}\right)
$$

and

$$
\bar{\partial}_{\Gamma^{\prime}} \bar{\partial}_{\Gamma^{\prime}}^{*}: \Omega^{1,0}\left(\Sigma ; N^{\otimes k} \otimes L_{\ell}\right) \rightarrow \Omega^{1,0}\left(\Sigma ; N^{\otimes k} \otimes L_{\ell}\right) .
$$

(Here $N_{k}$ has the complex structure induced by the connection $i \eta$.) The Hodge identities imply that these operators are the restriction of the Hodge Laplacian to the given space, and hence we have

$$
\partial_{\Gamma^{\prime}} \partial_{\Gamma^{\prime}}^{*} b^{\prime \prime}=\left(\lambda^{2}+\xi \lambda+\xi \zeta-\zeta^{2}\right) b^{\prime \prime},
$$

and

$$
\bar{\partial}_{\Gamma^{\prime}} \bar{\partial}_{\Gamma^{\prime}}^{*} b^{\prime}=\left(\lambda^{2}+\xi \lambda-\xi \zeta-\zeta^{2}\right) b^{\prime},
$$

where $\xi=\frac{n}{V}$ and $\zeta=\left(k-\frac{\ell}{n}\right)$. Since both of these operators are semipositive and we have dealt with the case where either of $b^{\prime}$ or $b^{\prime \prime}$ is in the kernel of the corresponding operator, we must have

$$
0<\lambda^{2}+\xi \lambda+\xi \zeta-\zeta^{2}
$$

and

$$
0<\lambda^{2}+\xi \lambda-\xi \zeta-\zeta^{2}
$$

The roots of the left-hand side of the first of these inequalities are $-\xi+\zeta$ and $-\zeta$ while for the second inequality the roots are $-\xi-\zeta$ and $\zeta$, so we must have $\lambda<-\left|k-\frac{\ell}{n}\right|$ or $\lambda>\left|k-\frac{\ell}{n}\right|$ as required. q.e.d.

We now turn to the proof of the correspondence theorems 3.8-(3.11). Let $A$ be a smooth finite energy ASD connection in $\mathfrak{g}_{E}$ over $\mathbb{R}_{ \pm} \times Y$. 
According to [36] we can find $T$ so that after gauge transformation we can assume that

$$
\left.A\right|_{[T, \infty) \times Y}=\Gamma+\gamma_{A}(t)+a(t),
$$

where $\Gamma$ is an $S^{1}$-invariant flat connection, $\gamma_{A}(t)$ is a flow line in a center manifold for the Chern-Simons flow through $\Gamma$ and $a(t)$ is an exponentially decaying one-form. By Lemma 3.13, we can arrange that $\gamma_{A}(t)$ is $S^{1}$-invariant.

Case 1: $r_{ \pm}([A])=[\Gamma] \in \mathscr{R}_{\mathrm{ev}}^{w}$. We can choose $\Gamma$ to be the pull-back of $\Gamma^{\prime}$, a flat connection with determinant $w$ on $E^{\Sigma} \rightarrow \Sigma$. If we are working with $\mathbb{R}_{+} \times Y$ then according to [36], the flow in the center manifold is constant regardless of whether or not $\Gamma^{\prime}$ is a smooth point of $\mathscr{R}(\Sigma)$. So we have $\gamma_{A}(t)=0$ and hence $\left.A\right|_{[T, \infty) \times Y}=\Gamma+a(t)$. Thus Guo's extension theorem applies to give a holomorphic structure $\hat{\mathcal{E}}_{A}$ on a bundle $\hat{E} \rightarrow N_{+}$extending $\mathcal{E}_{A}$. Furthermore the restriction of $\hat{\mathcal{E}}_{A}$ to $\Sigma_{+}$is equivalent to the holomorphic structure induced by $\Gamma^{\prime}$. If we are working with $\mathbb{R} \times Y$ and $\Gamma$ is irreducible, then $\Gamma$ is a smooth point of $\mathscr{R}_{\mathrm{ev}}^{w}$, so the flow in the center manifold is trivial and the argument proceeds as above. If $\Gamma$ is reducible, the flow in the center manifold is non-trivial. The term $\gamma_{A}$ in (3.17) is therefore present, and is not exponentially decaying in general; but it is $S^{1}$-invariant, so by Lemma 3.15 there is an $S^{1}$-invariant complex gauge transformation $h$ of $E$ on $(-\infty, T]$, so that $h(T)=1$ and

$$
h^{*}\left(\Gamma+\gamma_{A}\right)=\Delta,
$$

where $\Delta$ is a connection pulled back from $\mathscr{E}^{\Sigma} \rightarrow \Sigma$. Note that $\Delta$ is not necessarily a flat connection: if $\Gamma+\gamma_{A}$ is written in the form used in Lemma 3.15 , then $\Delta$ is the connection $B(T)$. We now have

$$
h^{*}\left(\Gamma+\gamma_{A}+a\right)=\Delta+a^{\prime},
$$

where $a^{\prime}$ is exponentially decaying. Theorem 3.12 applies to this connection to give a holomorphic structure $\hat{\mathcal{E}}_{A}$ extending $\mathcal{E}_{A}$. But now the restriction of the holomorphic structure to $\Sigma_{-}$is equivalent to that induced by $\Delta=B(T)$. The proof of Lemma 3.15 shows that $B(t)$ stays in a single $\mathscr{G}_{\mathbb{C}}$ orbit for all $t$, and approaches $\Gamma$ as $t \rightarrow-\infty$. The most we can say, therefore, is that $\Gamma$ is in the closure of the $\mathcal{G}_{\mathbb{C}}$ orbit through $\Delta ;$ that is to say, the holomorphic structures are $s$-equivalent. 
Case 2: $r_{ \pm}([A])=[\Gamma] \in \mathscr{R}_{\mathrm{od}}^{w}$. This case is almost the same at Case 1 , but the limiting connection $\Gamma$ is $\pi^{*}\left(\Gamma^{\prime}\right)+ \pm \operatorname{Diag}(i, i) \eta$ which is not pulled back from $\Sigma$. Let $h_{0}$ be the $\mathcal{G}_{\mathbb{C}}$ gauge transformation

$$
h_{0}=\operatorname{Diag}\left(r^{ \pm \frac{1}{2}}, r^{ \pm \frac{1}{2}}\right) .
$$

Then $h_{o}^{*}(\Gamma)=\pi^{*}\left(\Gamma^{\prime}\right)$. The argument now continues as in Case 1.

Case 3: $[\Gamma] \in \mathscr{R}_{m}^{w}$. Every point of $\mathscr{R}_{m}^{w}$ is a smooth point so $\gamma_{A}=0$ and $A$ decays exponentially to $\Gamma$. The argument breaks up into various subcases. Suppose first that we are working on $\mathbb{R}_{+} \times Y$, and $0<m \leq$ $n / 4$. Then

$$
\Gamma=\operatorname{Diag}\left(\pi^{*}\left(\Gamma_{m_{1}}^{\prime}\right), \pi^{*}\left(\Gamma_{m_{2}}^{\prime}\right)\right)+\operatorname{Diag}\left(-i \frac{m}{n}, i \frac{m}{n}\right) \eta,
$$

where $\Gamma_{m_{1}}^{\prime}$ and $\Gamma_{m_{2}}^{\prime}$ are Yang-Mills connections on line bundles of first Chern classes $m_{1}$ and $m_{2}$ respectively and where $0<m_{1}-m_{2}<\frac{n}{2}$ and $m=\frac{1}{2}\left(m_{1}-m_{2}\right)$. Let $\mathcal{L}_{m_{1}}$ and $\mathcal{L}_{m_{2}}$ denote the corresponding holomorphic structures. Again $\Gamma$ is not pulled back from $\Sigma$. It follows from Proposition 3.16 that an ASD connection asymptotic to $\Gamma$ has an asymptotic expansion as $t \rightarrow \infty$

$$
A(t)=\Gamma+\left[\begin{array}{rr}
0 & -\bar{b} \\
b & 0
\end{array}\right] e^{-\frac{\ell}{n} t}+c^{o}+c^{\perp},
$$

where $\ell=2 m, c^{o}$ is diagonal and has exponential decay, while $c^{\perp}$ is off-diagonal and and has exponential decay faster than $e^{-\frac{e}{n} t}$, or in polar coordinates vanishes faster than $r^{\frac{\ell}{n}}$. The form $b$ is the pull-back from $\Sigma$ of a $(1,0)$-form with values in $\mathcal{L}_{m_{2}} \otimes \mathcal{L}_{m_{1}}^{*}$. Let $h_{0}$ be the complex gauge transformation defined by the equation

$$
h_{0}=\operatorname{Diag}\left(r^{\frac{m}{n}}, r^{-\frac{m}{n}}\right) .
$$

Then $h_{0}^{*} \Gamma=\operatorname{Diag}\left(\pi^{*}\left(\Gamma_{m_{1}}^{\prime}\right), \pi^{*}\left(\Gamma_{m_{2}}^{\prime}\right)\right)$ and so

$$
h_{0}^{*}(A)=\operatorname{Diag}\left(\pi^{*}\left(\Gamma_{m_{1}}^{\prime}\right), \pi^{*}\left(\Gamma_{m_{2}}^{\prime}\right)\right)+\left[\begin{array}{rr}
0 & -\bar{b} \\
b & 0
\end{array}\right]+c^{o}+d^{\perp}
$$

where $d^{\perp}$ is off-diagonal and exponentially decaying. We can now apply Guo's theorem to get the extension. In this case the restriction of 
the holomorphic structure $\hat{\mathcal{E}}_{A}$ to $\Sigma$ is the extension of $\mathcal{L}_{m_{2}}$ by $\mathcal{L}_{m_{1}}$ determined by $-\bar{b}$ as required.

If we are working on $\mathbb{R}_{+} \times Y$ and $n / 4 \leq m<n / 2$ then we can write $\Gamma$ as above but the asymptotic expansion of $A(t)$ takes the form

$$
A(t)=\Gamma+\left[\begin{array}{rr}
0 & b \\
-\bar{b} & 0
\end{array}\right] e^{\frac{\ell}{n} t}+c^{o}+c^{\perp}
$$

where $\ell=n-2 m<0, c^{o}$ is diagonal and has exponential decay, while $c^{\perp}$ is off-diagonal and has exponential decay faster than $e^{\frac{\ell}{n} t}$, or in polar coordinates vanishes faster than $r^{\frac{-\ell}{n}}$. In this case the one form $b$ is the pull-back from $\Sigma$ of a holomorphic one-form with values in the conjugate bundle of $\mathcal{N} \otimes \mathcal{L}_{m_{2}}^{*} \otimes \mathcal{L}_{m_{1}}$, where $\mathcal{N}$ is the holomorphic structure in the normal bundle of $\Sigma_{+}$determined by the connection induced by $\eta$. Let $h_{0}$ be the complex gauge transformation defined by the equation

$$
h_{0}=\operatorname{Diag}\left(r^{-\frac{m}{n}}, r^{1+\frac{m}{n}}\right) .
$$

Then $h_{0}^{*} \Gamma=\operatorname{Diag}\left(\pi^{*}\left(\Gamma_{m_{1}}^{\prime}\right), \pi^{*}\left(\Gamma_{m_{2}}^{\prime} \otimes(i \eta)\right)\right)$ and so

$$
h_{0}^{*}(A)=\operatorname{Diag}\left(\pi^{*}\left(\Gamma_{m_{1}}^{\prime}\right), \pi^{*}\left(\Gamma_{m_{2}}^{\prime} \otimes(i \eta)\right)\right)+\left[\begin{array}{rr}
0 & b \\
-\bar{b} & 0
\end{array}\right]+c^{o}+d^{\perp}
$$

where $d^{\perp}$ is off-diagonal and exponentially decaying. We can now apply Guo's theorem to get the extension. In this case the restriction of the holomorphic structure $\hat{\mathcal{E}}_{A}$ to $\Sigma$ is the extension of $\mathcal{L}_{m_{1}}$ by $\mathcal{L}_{m_{2}} \otimes \mathcal{N}$ determined by $b$ as required. The cases involving $\mathbb{R} \times Y$ are entirely analogous and are left to the reader.

Combining these three cases implies the existence of the required extensions for each of the propositions (3.8)-(3.11). To determine the characteristic number $c$, notice that only in the third case do the complex gauge transformations change the value of the integral; in that case, the change is determined by a Chern-Simons invariant and the proof of Lemma 3.4 of [27] easily gives the desired result. It is straightforward to check that the determinant for the extension can be chosen as claimed. q.e.d.

(iii) Tunneling and elementary modification. Using the correspondence results above, we now analyze $\kappa$-relatedness. When $\kappa$ is small, this turns out to be closely related to the notion of elementary 
modification of bundles on a curve. We therefore begin with a discussion of elementary modification. This is standard material.

Let $\Sigma$ be an algebraic curve and let $\mathscr{E}$ and $\mathscr{E}^{\prime}$ be two rank-2 holomorphic bundles. Let $f: \mathscr{E}^{\prime} \rightarrow \mathscr{E}$ be map of sheaves which is an isomorphism at the generic point. As a map of sheaves, $f$ is then injective. Let

$$
D=\{\operatorname{det} f=0\}
$$

be the effective divisor defined by the vanishing of $\operatorname{det} f$. We regard $D$ as a zero-dimensional subscheme of $\Sigma$, possibly non-reduced. Since $f$ is injective we have an exact sequence

$$
\mathscr{E}^{\prime} \stackrel{f}{\longrightarrow} \mathscr{E} \longrightarrow \mathscr{Q} .
$$

The support of the quotient sheaf $\mathscr{Q}$ is contained in $D$. Looking at the holomorphic Euler characteristics of the sheaves in the exact sequence above, we also see that the dimension of $H^{0}(\mathscr{Q})$ is equal to the difference in the degrees of $\mathscr{E}^{\prime}$ and $\mathscr{E}$, which is also the degree of $D$. The following definition is not quite standard.

Definition 3.18. In the above situation, we say that $\mathscr{E}$ is obtained from $\mathscr{E}^{\prime}$ by elementary modification along $D$. If $\mathscr{Q}$ is a locally free $\mathscr{O}_{D}$-module (so that $\mathscr{Q}=\mathscr{O}_{D}$ in fact), we say that the modification is regular.

For now, consider the case that $D$ is just a multiple of a point $P$. That is, $D=n P$ and the structure ring of $D$ is

$$
\mathscr{O}_{n P}=\mathbb{C}[z] / z^{n} .
$$

The sheaf $\mathscr{Q}$ is an $n$-dimensional module over this ring. Such a sheaf is precisely an $n$-dimensional complex vector space with a nilpotent endomorphism. These are classified by partitions of $n$ via their Jordan canonical form. Thus we see that

$$
\mathscr{Q}=\bigoplus \mathscr{O}_{m_{\boldsymbol{i}} P}
$$

where $m_{i}$ is a partition of $n$. The regular case is when there is a single Jordan block of size $n$; in the non-regular case, the support of $\mathscr{Q}$ is contained in $m P$ for some $m<n$.

In general, if we exhibit $\mathscr{Q}$ as an extension of $\mathscr{O}_{n P}$-modules, say

$$
\mathscr{Q}_{0} \rightarrow \mathscr{Q} \rightarrow \mathscr{Q}_{1},
$$


then we can regard $\mathscr{E}$ as being obtained from $\mathscr{E}^{\prime}$ in two stages, via an intermediate $\mathscr{E}_{1}$ :

$$
\begin{aligned}
& \mathscr{E}^{\prime} \rightarrow \mathscr{E}_{1} \rightarrow \mathscr{Q}_{0} \\
& \mathscr{E}_{1} \rightarrow \mathscr{E}^{\rightarrow} \rightarrow \mathscr{Q}_{1} .
\end{aligned}
$$

Conversely, if $\mathscr{E}$ is obtained in two stages from $\mathscr{E}^{\prime}$ in this way, then $\mathscr{E}$ is clearly also obtainable from $\mathscr{E}^{\prime}$ in one stage, with cokernel an extension of $\mathscr{Q}_{1}$ by $\mathscr{Q}_{0}$. Because of the exact sequence $\mathscr{O}_{P} \rightarrow \mathscr{O}_{m P} \rightarrow \mathscr{O}_{(m-1) P}$, we can reduce any elementary modification to a sequence of modifications of the form

$$
\mathscr{E}_{1} \rightarrow \mathscr{E} \rightarrow \mathscr{O}_{P}
$$

i.e. a sequence of elementary modifications at closed points. Thus we have shown:

Lemma 3.19. If $D$ is a divisor of degree $N$, then an elementary modification along $D$ is the same as a sequence of $N$ elementary modifications at closed points $P_{i}$, where $\sum P_{i}=D$.

The next Lemma is equally elementary:

Lemma 3.20. If $\mathscr{E}$ is obtained from $\mathscr{E}^{\prime}$ by elementary modification along $D$, then $\mathscr{E}^{\prime}[D]$ can be obtained from $\mathscr{E}$ also by elementary modification along $D$.

The following proposition shows that if a holomorphic bundle $\mathscr{E}$ on the ruled surface $W$ has $c(\mathscr{E})$ sufficiently small, then the restrictions of $\mathscr{E}$ to the curves $\Sigma_{+}$and $\Sigma_{-}$are related by elementary modification. Similar results are contained in [21] and [22].

Proposition 3.21. Let $\mathscr{E} \rightarrow W$ be a rank-2 holomorphic bundle, and let $c=c(\mathscr{E})$. Let $\mathscr{E}_{+}$and $\mathscr{E}_{-}$be the bundles on $\Sigma$ obtained by restricting $\mathscr{E}$ to the curves $\Sigma_{+}$and $\Sigma_{-}$. Suppose $\mathscr{E}_{+}$admits no linesubbundle of degree greater than $m+\frac{1}{2} \operatorname{deg} \mathscr{E}_{+}$, for some $m \leq n / 2$.

(a) If

$$
c+m^{2} / n<n\left(\frac{1}{2}-m / n\right)^{2},
$$

then $c_{1}(\mathscr{E})[F]$ is even. (Here $F$ is again the fibre.)

(b) If $c_{1}(\mathscr{E})[F]$ is even, so $c_{1}(\mathscr{E})[F]=2 p$ say, and

$$
c+m^{2} / n<n(1-m / n)^{2},
$$

let $\mathscr{L}=\left[-p \Sigma_{-}\right]$, so that $c_{1}(\mathscr{E} \otimes \mathscr{L})[F]=0$. Then there is an effective divisor $D$ in $\Sigma$ of degree at most $c$ and a holomorphic 
bundle $\mathscr{E}_{0}$ on $\Sigma$ such that both $\mathscr{E}_{+}$and $\left.\mathscr{E}_{-} \otimes \mathscr{L}\right|_{\Sigma_{-}}$are obtained from $\mathscr{E}_{0}$ by elementary modification (possibly non-regular) along $D$.

The rest of this subsection contains the proof of this proposition. A rank-2 holomorphic bundle on $\mathbb{C P}^{1}$ splits as $\mathscr{O}(p) \oplus \mathscr{O}(q)$ for some $p$ and $q$, by Birkhoff's theorem. For a holomorphic bundle $\mathscr{E}$ on $W$, one can examine the splitting type on each fibre. On the complement of a finite set of fibres, the splitting type will be constant: this is the generic splitting type of $\mathscr{E}$. We also make the following definition:

Definition 3.22. Let $\mathscr{E}$ be a rank-2 bundle on $\mathbb{C P}^{1}$, isomorphic to $\mathscr{O}(p) \oplus \mathscr{O}(q)$, say. The splitting degree of $\mathscr{E}$ is the number $r=\frac{1}{2}|p-q| \epsilon$ $\frac{1}{2} \mathbb{Z}$. For example, $\mathscr{O}(r) \oplus \mathscr{O}(-r)$ has splitting degree $r$, while $\mathscr{O}(1) \oplus \mathscr{O}$ has splitting degree $\frac{1}{2}$.

Lemma 3.23. Let $\mathscr{E}$ be a rank-2 bundle on the ruled surface $W$ with the property that $\left.\mathscr{E}\right|_{\Sigma_{+}}$admits no line sub-bundle of degree greater than $m+\frac{1}{2} \operatorname{deg}\left(\left.\mathscr{E}\right|_{\Sigma_{+}}\right)$, for some number $m$. Let $r$ be the splitting degree of $\mathscr{E}$ on the generic fibre. If $r$ is non-zero, then

$$
c(\mathscr{E})+m^{2} / n \geq n(r-m / n)^{2} .
$$

Proof. We illustrate the algebra only with the case that $c_{1}(\mathscr{E})=0$. If the generic splitting is $\mathscr{O}(r) \oplus \mathscr{O}(-r)$ with $r>0$, then we have a distinguished line-subbundle on the generic fibre. This extends to give us a line bundle $\mathscr{F} \rightarrow W$ and a map

$$
f: \mathscr{F} \rightarrow \mathscr{E},
$$

which we may assume is an injective map of bundles except at isolated points. Thus there is an exact sequence

$$
\mathscr{F} \rightarrow \mathscr{E} \rightarrow \Lambda^{2} \mathscr{E} \otimes \mathscr{F}^{-1} \otimes \mathcal{I}_{Z}
$$

where $Z$ is a zero-scheme. We have $c_{1}(\mathscr{F})[F]=r$, so

$$
c_{1}(\mathscr{F})=r\left[\Sigma_{-}\right]+a[F]
$$

From the condition on $\left.\mathscr{E}\right|_{\Sigma_{+}}$, we have $a \leq m$. From the exact sequence 
above, we have

$$
\begin{aligned}
c(\mathscr{E})+m^{2} / n & =-c_{1}^{2}(\mathscr{F})+l(Z)+m^{2} / n \\
& =r^{2} n-2 a r+l(Z)+m^{2} / n \\
& \geq r^{2} n-2 m r+m^{2} / n \\
& =n(r-m / n)^{2}
\end{aligned}
$$

since $r$ and $l$ are positive. q.e.d.

Now let us return to the situation of Proposition 3.21. If the degree of $\mathscr{E}$ on the fibre $F$ is odd, then the splitting degree $r$ on the generic fibre is in $\frac{1}{2}+\mathbb{Z}$, and is therefore non-zero. The hypothesis on $\mathscr{E}_{+}$fits the above Lemma, and we conclude that

$$
c(\mathscr{E})+m^{2} / n \geq n(r-m / n)^{2} .
$$

Because $m$ is supposed to be less than $n / 2$, and $r \geq \frac{1}{2}$, the righthand side is not less than $n\left(\frac{1}{2}-m / n\right)^{2}$. This proves part $(a)$ of the Proposition.

Replacing $\mathscr{E}$ by $\mathscr{E} \otimes \mathscr{L}$ if necessary, we may assume in part (b) that $c_{1}(\mathscr{E})$ is zero on $F$. The generic splitting degree $r$ is now a nonnegative integer, and the upper bound on $c(\mathscr{E})$ in the hypotheses of part $(b)$ forces $r$ to be zero, by the Lemma above. So $\mathscr{E}$ is trivial on the generic fibre. Let $\pi$ be the projection of $W$ onto either $\Sigma_{+}$or $\Sigma_{-}$; the two cases are much the same, and we focus on $\Sigma=\Sigma_{+}$. The direct image sheaf $\mathscr{E}^{\prime}=\pi_{*}(\mathscr{E})$ on $\Sigma$ is isomorphic to $\mathscr{E}_{\Sigma_{+}}=\mathscr{E}_{+}$wherever the splitting degree is trivial. The direct image is also torsion free, since $\mathscr{E}$ is torsion free. The sheaf $\mathscr{E}^{\prime}$ is therefore a rank-2 vector bundle on $\Sigma$.

There is a tautological inclusion of $\pi^{*}\left(\mathscr{E}^{\prime}\right)$ in $\mathscr{E}$ as sheaves, and this is an isomorphism except at the exceptional fibres where $\mathscr{E}$ splits nontrivially. So we have a map between vector bundles

$$
f: \pi^{*}\left(\mathscr{E}^{\prime}\right) \rightarrow \mathscr{E}
$$

on $W$, and the divisor $\{\operatorname{det} f=0\}$ is $\pi^{*}(D)$ for some divisor $D$ on $\Sigma$. We can estimate the degree of $D$ using the Grothendieck-RiemannRoch theorem. We shall treat only the case that $c_{1}(\mathscr{E})=0$ on $W$. In this case, we have

$$
\operatorname{deg} D=-c_{1}\left(\mathscr{E}^{\prime \prime}\right)[\Sigma]
$$

Let $R^{1} \pi_{*} \mathscr{E}$ be the first higher direct image of $\mathscr{E}$. This is a sheaf on $\Sigma$ supported in the neighbourhood of the divisor $D$. The difference 
$\mathscr{E}^{\prime}-R^{1} \pi_{*} \mathscr{E}$ defines an element $\sigma$ in the $K$-theory of $\Sigma$, which corresponds to the index of the family of $\bar{\partial}$-operators. We calculate:

$$
\begin{aligned}
\operatorname{ch}(\sigma) & =\operatorname{ch}(\mathscr{E}) \operatorname{Td}\left(T_{*} F\right) /[F] \\
& =\left(2-c_{2}(\mathscr{E})\right)(1+[F]) /[F] \\
& =2-c_{2}(\mathscr{E}) /[F] .
\end{aligned}
$$

So $c_{1}(\sigma)[\Sigma]=-c_{2}(\mathscr{E})[W]$, and hence

$$
\operatorname{deg}(D)=c_{2}(\mathscr{E})-h^{0}\left(R^{1} \pi_{*}(\mathscr{E})\right) .
$$

Thus the degree of $D$ is at most $c=c_{2}(\mathscr{E})$.

Restricting the map $f$ to $\Sigma_{+}$, we see that there is a map $f: \mathscr{E}^{\prime} \rightarrow \mathscr{E}_{+}$ whose determinant vanishes on the divisor $D$. Thus $\mathscr{E}_{+}$is obtained from $\mathscr{E}^{\prime}$ by an elementary modification along $D$. Restricting $f$ to $\Sigma_{-}$, we see the same thing for $\mathscr{E}_{-}$. This completes the proof of Proposition 3.21. q.e.d.

(iv) Proof of the dimension estimates. We now set out the proofs of Theorem 3.2 and Propositions 3.4-3.6, using the results of the previous two subsections.

Proof of Theorem 3.2. Suppose $\rho$ and $\sigma$ are in $\mathscr{R}_{\mathrm{ev}}^{w, s}$ and $\rho \bumpeq_{k} \sigma$, for some $k \leq n$, as in the Theorem. (The case $\sigma \bumpeq_{k} \rho$ will be no different.) Then there is an anti-self-dual connection $A$ on $\mathbb{R} \times Y$ with $r_{-}(A)=\rho$, $r_{+}(A)=\sigma$ and $c(A) \leq k$.

Let $\mathscr{E}_{\rho}$ and $\mathscr{E}_{\sigma}$ be the holomorphic bundles on $\Sigma$ corresponding to $\rho$ and $\sigma$. Proposition 3.8 provides a holomorphic bundle $\mathscr{E} \rightarrow W$ with $\operatorname{det}(\mathscr{E})=\omega$ and $c(\mathscr{E})=c_{2}(\mathscr{E})=c(A)$, such that the restrictions $\left.\mathscr{E}\right|_{\Sigma_{-}}$ and $\left.\mathscr{E}\right|_{\Sigma_{+}}$are isomorphic to $\mathscr{E}_{\rho}$ and $\mathscr{E}_{\sigma}$ respectively. (There is strict isomorphism at $\Sigma_{-}$rather than just $s$-equivalence because $\mathscr{E}_{\rho}$ is stable, not just semi-stable.)

Because $\mathscr{E}_{\sigma}$ is stable, the hypotheses of Proposition 3.21 are satisfied with $m=-1$ : that is, $\mathscr{E}_{\sigma}=\left.\mathscr{E}\right|_{\Sigma_{+}}$has no line-subbundle with normalized degree $\operatorname{deg}_{\omega}(\mathscr{L})$ greater than -1 . The hypothesis $k \leq n$ is more than enough to ensure that

$$
c+m^{2} / n<n(1-m / n)^{2}
$$

when $m=-1$, so part $(b)$ of Proposition 3.21 applies: there is a bundle $\mathscr{E}_{0}$ and a divisor $D$ of degree at most $k$ such that both $\mathscr{E}_{\rho}$ and $\mathscr{E}_{\sigma}$ 
are obtained from $\mathscr{E}_{0}$ by elementary modification along $D$. Using Lemmas 3.19 and 3.20 , we can rephrase this conclusion by saying that $\mathscr{E}_{\rho}$ is obtained from $\mathscr{E}_{\sigma}[-D]$ by elementary modification along $2 D$. Using the same lemmas, we see that we can arrange that $D$ has degree $k$ exactly.

We now build a manifold $U_{k}$ as required in the Theorem. First, given a bundle $\mathscr{E}^{\prime}$ and a divisor $D$ written as an ordered sum

$$
D=P_{1}+\cdots+P_{d},
$$

we build a manifold $E M\left(\mathscr{E}^{\prime}, D\right)$ whose points correspond to data specifying an elementary modification of $\mathscr{E}^{\prime}$ along $D$. Such a space can be constructed as an iterated $\mathbb{C P}^{1}$ bundle using Lemma 3.19 , because the data needed to effectively specify an elementary modification at a single point $P$ is a point of a $\mathbb{C P}^{1}$.

Define $\bar{U}_{k}$ to be the total space of the smooth fibre bundle

$$
p: \bar{U}_{k} \longrightarrow \mathscr{R}_{\mathrm{ev}}^{w, s} \times \Sigma^{k}
$$

whose fibre over $\left(\sigma,\left(P_{1}, \ldots, P_{k}\right)\right)$ is $\operatorname{EM}\left(\mathscr{E}_{\sigma}[-D], 2 D\right)$, where $D=$ $\sum P_{i}$. The base has dimension $6 g-6+2 k$ and the fibre has dimension $4 k$, since it is a $2 k$-fold iterated $\mathbb{C P}^{1}$ bundle, so $\bar{U}_{k}$ has dimension $6 g-6+6 k$. To each point of $u \in \bar{U}_{k}$, we can assign two bundles $\mathscr{E}_{1}$ and $\mathscr{E}_{2}:$ if $u$ lies in the fibre $\operatorname{EM}\left(\mathscr{E}_{\sigma}[-D], 2 D\right)$ over $(\sigma, D)$, then we take $\mathscr{E}_{2}$ to be $\mathscr{E}_{\sigma}$, and $\mathscr{E}_{1}$ to be the result of the elementary modification to $\mathscr{E}_{2}[-D]$ which $u$ specifies. Let $U_{k} \subset \bar{U}_{k}$ be the open subset on which $\mathscr{E}_{1}$ is stable. On this subset, $\mathscr{E}_{1}$ and $\mathscr{E}_{2}$ provide maps $t_{1}$ and $t_{2}$ from $U_{k}$ to $\mathscr{R}_{\mathrm{ev}}^{w, s}$. The statement that $\mathscr{E}_{\rho}$ is obtained from $\mathscr{E}_{\sigma}[-D]$ by elementary modification along $2 D$ is now the same as saying that $(\rho, \sigma)$ is in the image of $t=\left(t_{1}, t_{2}\right)$, as required. The case that $\rho$ and $\sigma$ belong to $\mathscr{R}_{\mathrm{od}}^{w, s}$ is not essentially different. q.e.d.

Proof of Proposition 3.4. Suppose $\rho \bumpeq_{\kappa} \sigma$ with $\rho \in \mathscr{R}_{\mathrm{ev}}^{w}$ and $\sigma \in \mathscr{R}_{\mathrm{od}}^{w}$. (The case $\sigma \bumpeq_{\kappa} \rho$ is little different.) Then, by Proposition 3.9 there is an $\mathscr{E} \rightarrow W$ with $c(\mathscr{E}) \leq \kappa$ whose restriction $\left.\mathscr{E}\right|_{\Sigma_{+}}$is isomorphic to $\mathscr{E}_{\sigma}$. Because $\rho$ and $\sigma$ belong to different components, $c_{1}(\mathscr{E})[F]$ is odd. The semi-stability of $\left.\mathscr{E}\right|_{\Sigma_{+}}$means that we can apply Proposition 3.21 with $m=0$. Part $(a)$ of the Proposition tells us that $\kappa$ must be at least $n / 4$. So, if $\kappa$ is less than $n / 4$, we cannot have $\rho \bumpeq_{k} \sigma$. q.e.d.

Proof of Proposition 3.5. Suppose $\sigma \in \mathscr{R}_{\mathrm{ev}}^{w, s i n g}$ and $\rho \bumpeq_{k} \sigma$, with $\rho \in \mathscr{R}_{\mathrm{ev}}^{w, s}$ and $k<n$. Let $\mathscr{E} \rightarrow W$ be the bundle with $\operatorname{det}(\mathscr{E})=\omega$ 
and $c(\mathscr{E}) \leq k$ supplied by Proposition 3.8 , so that $\mathscr{E}_{\Sigma_{-}}=\mathscr{E}_{\rho}$ and $\left.\mathscr{E}\right|_{\Sigma_{+}}=\mathscr{E}_{\sigma}$. Again, there is an isomorphism at the $\Sigma_{-}$end rather than $s$-equivalence because $\rho$ is irreducible. Because $\mathscr{E}_{\sigma}$ is semi-stable, we can apply Proposition 3.21 with $m=0$; part $(b)$ is the relevant part, since $c_{1}(\mathscr{E})[F]=0$. We conclude that, as $c(\mathscr{E}) \leq k<n$, the bundle $\mathscr{E}_{\rho}$ is obtained from $\mathscr{E}_{\sigma}[-D]$ by elementary modification along $2 D$, for some divisor $D$ of degree $k$. As in the proof of (3.2), the set of all bundles on $\Sigma$ obtainable from a fixed $\mathscr{E}_{\sigma}$ by such a modification can be parametrized (not necessarily effectively) by a $2 k$-fold iterated $\mathbb{C P}^{1}$ bundle over $\Sigma^{k}$. That is to say, if we write $D$ as an ordered sum,

$$
D=P_{1}+P_{2}+\cdots+P_{k}
$$

and write

$$
2 D=P_{1}+P_{1}+P_{2}+P_{2}+\cdots+P_{k},
$$

then we have a $\mathbb{C P}^{1}$ 's worth of choice in making successive modifications at $P_{1}, P_{1}, P_{2}, P_{2}$ and so on. However, because $\mathscr{E}_{\sigma}$ is reducible, the first $\mathbb{C P}^{1}$ is not effective: there is a $\mathbb{C}^{*}$ acting by automorphisms of $\mathscr{E}_{\sigma}$ which has three orbits in its standard action on $\mathbb{C P}^{1}$. Therefore, the bundles obtainable from $\mathscr{E}_{\sigma}$ in this way can be parametrized (still not effectively perhaps) by three bundles over $\Sigma^{k}$ with fibres which are $(2 k-1)$-fold iterated $\mathbb{C P}^{1}$ bundles. Amongst this family, the stable bundles form an analytic family of real dimension $6 k-2$.

This shows that $\overleftarrow{T}_{k}\{\sigma\} \cap \mathscr{R}_{\mathrm{ev}}^{w, s}$ is contained in dimension $6 k-2$. If we consider more generally a subset $K$ in $\mathscr{R}_{\mathrm{ev}}^{w, s i n g}$ covered by a smooth map $f: Z \rightarrow \mathscr{R}_{0}^{w}$, where $Z$ has dimension $\delta$, it is straightforward to build a family over $Z$ of dimension $\delta-2+6 k$ which covers $\overleftarrow{T}_{k}(K) \cap \mathscr{R}_{\mathrm{ev}}^{w, s}$. Once again, replacing the even component by the odd one does not lead to any difficulty. q.e.d.

Proof of Proposition 3.6. Parts $(a)$ and $(b)$ of this Proposition are very much the same. Indeed, when $n$ is even the two statements are entirely equivalent, for in this case there is a real line-bundle $\xi$ on $Y$ with holonomy -1 on the fibre, and tensoring by $\xi$ interchanges $\mathscr{R}_{\mathrm{ev}}^{w}$ with $\mathscr{R}_{\mathrm{od}}^{w}$ and $\mathscr{R}_{m}^{w}$ with $\mathscr{R}_{m^{\prime}}^{w}$. We will consider only part $(a)$ therefore. Furthermore, taking our lead from the paragraph above, we will consider only the case that $K \subset \mathscr{R}_{m}^{w}$ is a single point and $\delta=0$ : it will again be clear that the constructions can be carried out over any smooth family. 
So take $\sigma$ in $\mathscr{R}_{m}^{w}$ and suppose $\rho \bumpeq_{\kappa} \sigma$, with $\rho \in \mathscr{R}_{\mathrm{ev}}^{w, s}$. From Proposition 3.10 , we obtain a bundle $\mathscr{E} \rightarrow W$ with determinant $\omega$ and $c(\mathscr{E})+m^{2} / n \leq \kappa$. The restriction $\left.\mathscr{E}\right|_{\Sigma_{+}}$is an extension

$$
\left.\mathscr{L}_{-m} \rightarrow \mathscr{E}\right|_{\Sigma_{+}} \rightarrow \mathscr{L}_{m}
$$

where $\mathscr{L}_{-m}$ is the line-bundle corresponding to $\sigma$, with $\operatorname{deg}_{\omega} L_{-m}=-m$. From this sequence it follows that $\left.\mathscr{E}\right|_{\Sigma_{+}}$admits no line subbundle of normalized degree greater than $m$. Therefore Proposition 3.21 applies and tells us that, if $\kappa<n(1-m / n)^{2}$, then the generic splitting type of $\mathscr{E}$ is zero and $\left.\mathscr{E}\right|_{\Sigma_{-}}$(which is isomorphic to $\mathscr{E}_{\rho}$ ) is obtained from $\left.\mathscr{E}\right|_{\Sigma_{+}}[-D]$ by an elementary modification along $2 D$. Here $D$ can be taken to be an effective divisor of degree $c(\mathscr{E})$, which is at most $\kappa-m^{2} / n$; the Chern number $c(\mathscr{E})$ has to be non-negative in this situation, as $\mathscr{E}$ has trivial splitting type on the generic fibre.

We now count the dimension of a space which parametrizes a family of bundles on $\Sigma$ which includes all the possible bundles $\mathscr{E}_{\rho}$. First, the bundle $\left.\mathscr{E}\right|_{\Sigma_{+}}$is determined by the class of the extension (3.25), which belongs to a projective space $\mathbb{P} H^{1}\left(\Sigma,\left(\mathscr{L}_{-m}\right)^{2}\right)$, which has real dimension $2 g-4+4 m$. There are at most $2\left(\kappa-m^{2} / n\right)$ parameters in choosing the divisor $D$, and a further $4\left(\kappa-m^{2} / n\right)$ parameters of choice in specifying the elementary modification along $2 D$. The total count is therefore at most $2 g-4+4 m+6\left(\kappa-m^{2} / n\right)$, as claimed. q.e.d.

Remark. For each of the Propositions we have proved in this subsection, one can construct a variant considering families of metrics, along the following lines. The relation $\bumpeq_{\kappa}$ on $\mathscr{R}^{w}(Y)$ depends on the chosen Riemannian metric on $\Sigma$, through the corresponding complex structure. Consider a family of metrics on $\Sigma$, parametrized by a manifold $I$. Let $K$ be a subset of $I \times \mathscr{R}_{\mathrm{ev}}^{w, s}$, contained in dimension $\delta$, and fix $k \leq n$. Consider the subsets of $I \times \mathscr{R}_{\mathrm{ev}}^{w, s}$

$$
\bigcup_{(i, \rho) \in K}\left(\{i\} \times \vec{T}_{k, i}(\rho) \cap \mathscr{R}_{\mathrm{ev}}^{w, s}\right) \text { and } \bigcup_{(i, \rho) \in K}\left(\{i\} \times \overleftarrow{T}_{k, i}(\rho) \cap \mathscr{R}_{\mathrm{ev}}^{w, s}\right)
$$

where $\vec{T}_{k, i}$ and $\overleftarrow{T}_{k, i}$ are the relations defined by the metric corresponding to $i \in I$. A variant of Corollary 3.3 states that these two sets are both contained in dimension $\delta+6 k$. The only observation needed to verify this is that the construction of the family of bundles parametrized by $\bar{U}_{k}$ (see (3.24)) can also be used to construct a similar 
family parametrized by $I \times \bar{U}_{k}$. (Note that the underlying manifold of $\bar{U}_{k}$ is independent of the complex structure.) We leave the reader to formulate similar versions of the other propositions.

\section{Floer homology for circle bundles}

Let $(X, \Sigma)$ be a pair consisting of a 4-manifold and a smoothly embedded surface. Let $g$ be the genus, and suppose the self-intersection number $n=Q(\Sigma)$ is positive. Write $X^{o}$ for the open manifold $X / \Sigma$ equipped with a cylindrical end modelled on $Y=Y(n, g)$, and let $W^{o}$ be a tubular neighbourhood of $\Sigma$, which we can equip with a cylindrical end modelled on $\bar{Y}$. Suppose that $X^{\circ}$ is admissible in the sense of (2.14) and that the pair $(X, \Sigma)$ is admissible in the sense of (2.23). We aim here to describe a Floer homology theory which allows partial calculation of the invariants $D_{X}^{w}$ and $D_{X, \Sigma}^{w, p}$ in terms of relative invariants of the manifolds $X^{o}$ and $W^{o}$. The theory is 'partial' in that we impose restrictions on the dimensions of the moduli spaces involved. Subject to these restrictions, the invariants of $X^{o}$ and $W^{o}$ take values in the ordinary homology and cohomology (respectively) of the irreducible representation variety, $\mathscr{R}_{\mathrm{ev}}^{w, s}(Y) \amalg \mathscr{R}_{\mathrm{od}}^{w, s}(Y)$.

(i) Construction of cycles from maps with small frontier. We establish some notation for this section. Let $\Delta^{\delta}$ be the standard $\delta$-simplex. A singular $\delta$-simplex in a space $R$ is a map $\sigma: \Delta^{\delta} \rightarrow R$. A rational singular chain $c$ is a formal finite rational linear combination $c=\sum_{i=1}^{N} r_{i} \sigma_{i}$. The $\sigma_{i}$ are called the characteristic maps of $c$. We say that a singular chain in a smooth manifold is smooth if each of its characteristic maps is smooth. For a smooth manifold $R$, let $C_{\delta}(R)$ denote the space of smooth rational $\delta$-chains. A singular $\delta$-chain is transverse to a submanifold $S$ if the restriction of each of its characteristic maps to any face of $\Delta^{\delta}$ is transverse to $S$. More generally a pair of singular chains $c$ and $c^{\prime}$ of dimensions $\delta$ and $\delta^{\prime}$ are said to be transverse provided that the restriction of each characteristic map of $c$ to any face of $\Delta^{\delta}$ is transverse to each of the restrictions of the characteristic maps of $c^{\prime}$ to any face of $\Delta^{\delta^{\prime}}$.

Definition 4.1. Let $M$ be an oriented $\delta$-manifold and let $f: M \rightarrow R$ be a smooth map. We say that smooth singular chain $c$ agrees with $(M, f)$ outside an open set $U \subset R$ if there is a triangulation $\mathcal{T}$ of $M$ 
so that $c=\sum_{t \in \mathcal{T}} f \circ t$ as elements of $C_{\delta}(R, U)$.

Finally if $f: S \rightarrow R$ is a map, we define the frontier of $f$, denoted $\operatorname{Fr}(f)$, to be the set

$$
\left\{r \in R \mid r=\lim f\left(s_{i}\right) \text { and }\left(s_{i}\right) \text { has no convergent subsequence }\right\}
$$

This is the complement of the largest open set $U$ in $R$ such that $\left.f\right|_{f^{-1}(U)}$ is proper.

Proposition 4.2. Let $M$ be a smooth, oriented $\delta$-manifold and let $R$ be a compact smooth oriented manifold of dimension $n \geq \delta$ possibly with boundary. Suppose that $f: M \rightarrow R$ is a smooth map such that the closure of $f(M)$ is contained in $\operatorname{int}(R)$ and such that the frontier $\operatorname{Fr}(f)$ is contained dimension $\delta-2$. Then there is a neighborhood $U$ of $\operatorname{Fr}(f)$ with the following two properties. First, for every neighborhood $V$ of $\operatorname{Fr}(f)$ with $\bar{V} \subset U$, there is a rational singular smooth $\delta$-cycle $c \in C_{\delta}(R)$ which agrees with $(M, f)$ outside $V$. Secondly, any $\delta$-cycle which agrees with $(M, f)$ outside $U$ defines the same rational homology class as $c$. Thus $(M, f)$ gives rise to a well-defined homology class in $H_{\delta}(R ; \mathscr{Q})$, namely the class $[c]$ of any cycle agreeing with $(M, f)$ outside a sufficiently small neighbourhood of $\operatorname{Fr}(f)$.

Definition 4.3. We call the rational homology class produced by this lemma $[M, f] \in H_{\delta}(R ; \mathbb{Q})$.

Proof of 4.2. Since $\operatorname{Fr}(f)$ is contained in dimension $\delta-2$ there are manifolds $N_{1}, \ldots, N_{s}$ of dimension at most $\delta-2$ and smooth maps $g_{i}: N_{i} \rightarrow R$ so that $\cup_{i=1}^{t} g_{i}\left(N_{i}\right) \supset \operatorname{Fr}(f)$. For the neighborhood $U$ of $\operatorname{Fr}(f)$ we can take any neighborhood so that the following three properties hold: $\bar{U}$ is a smooth manifold with boundary, $f$ is transverse to $\partial U$, and every homology class in $H_{n-\delta}(R, \partial R ; \mathbb{Q})$ has a representative disjoint from $U$. To see that such neighborhoods exist we argue as follows. Since $\operatorname{Fr}(f)$ is a closed set, we can always find arbitrarily small neighborhoods satisfying the first two conditions. Since $R$ is a compact manifold possibly with boundary, its homology is finitely generated and we can find a basis for the homology in general position with respect to the maps $g_{1}, \ldots, g_{t}$. By the dimension hypothesis, this basis will miss $\operatorname{Fr}(f)$ and hence also a sufficiently small neighborhood of $\operatorname{Fr}(f)$.

Consider the $(\delta-1)$-dimensional rational homology class $h$ represented by $\left.f\right|_{f^{-1}(\partial U)}: f^{-1}(\partial U) \rightarrow R$. We claim that $h$ is in the image of 
the boundary map

$$
H_{\delta}(\bar{U}, \partial U ; \mathbb{Q}) \rightarrow H_{\delta-1}(\partial U ; \mathbb{Q}) .
$$

This will be the case if $h$ pairs trivially with the image of the restriction $\operatorname{map} H^{\delta-1}(\bar{U} ; \mathbb{Q}) \rightarrow H^{\delta-1}(\partial U ; \mathbb{Q})$; or equivalently, by Poincaré duality, if the intersection number of $h$ with every singular cycle in the image of

$$
H_{n-\delta}(\bar{U}, \partial U ; \mathbb{Q}) \rightarrow H_{n-\delta-1}(\partial U ; \mathbb{Q})
$$

is zero. Choose a cycle $s$ representing an element of $H_{n-\delta}(\bar{U}, \partial U ; \mathbb{Q})$ so that $\partial s$ is transverse to $h$. We can assume $s$ is transverse to $f$ and the $g_{i}$. Then the image of $s$ is disjoint from $\operatorname{Fr}(f)$. The fibered product $f^{-1}(\bar{U}) \times_{f} s$ defines a smooth 1-cycle with boundary $h \cap \partial s$ proving the claim. Thus $h=\partial k$ for some rational smooth singular $\delta$-cycle in $U$. Now take a chain $j$ which represents the homology class of $\left(f^{-1}(R \backslash U), f\right)$ so that $\partial j=h$ then $j-k \in C_{\delta}(R)$ is a cycle of the desired form.

It remains to prove the uniqueness assertion. Let $c$ and $c^{\prime}$ be cycles representing elements of $H_{\delta}(R ; \mathbb{Q})$ which agrees with $(M, f)$ outside $V$. To check that they are homologous it suffices, by Poincaré duality, to check that they have the same intersection number with elements of $H_{n-\delta}(R, \partial R ; \mathbb{Q})$. This is clear since, by construction, there is a basis for $H_{n-\delta}(R, \partial R ; \mathbb{Q})$ represented by cycles disjoint from $V$. q.e.d.

The next proposition gives a useful condition under which the cycles obtained from $\left(M_{0}, f_{0}\right)$ and $\left(M_{1}, f_{1}\right)$ are homologous.

Proposition 4.4. Let $f_{0}: M_{0} \rightarrow R$ and $f_{1}: M_{1} \rightarrow R$ be two maps satisfying the conditions of Proposition 4.2. Suppose that $W$ is a smooth, oriented $\delta+1$ manifold with boundary $\bar{M}_{0} \cup M_{1}$ and that $g: W \rightarrow R \times[0,1]$ is a smooth map with $\left.g\right|_{M_{i}}=f_{i}$. Suppose $g$ satisfies the following conditions.

(i) $\overline{(g(W))} \subset \operatorname{int}(R) \times I$.

(ii) $\operatorname{Fr}(g)$ is contained in dimension $\delta-1$.

(iii) $g$ is transverse to $R \times\{0,1\}$.

Then we have the equality of homology classes $\left[M_{0}, f_{0}\right]=\left[M_{1}, f_{1}\right]$.

Proof. We must show that the intersection numbers of $\left[M_{0}, f_{0}\right]$ and $\left[M_{1}, f_{1}\right]$ with cycles representing elements of $H_{n-\delta}(R, \partial R ; \mathbb{Q})$ agree. Let $s$ be such a cycle. We can assume that $s$ is transverse to $f_{0}$ and $f_{1}$ and disjoint from the frontier of these maps. The hypotheses of this 
proposition imply that a generic perturbation $\tilde{s}$ of the product cycle $s \times[0,1]$, relative to the boundary $s \times\{0,1\}$, will be disjoint from the frontier of $g$ and that the fibered product $W \times{ }_{g}(\tilde{s})$ is a 1-cycle. The boundary of $W \times_{g}(\tilde{s})$ is the fibered product $M_{1} \times_{f_{1}} s-M_{0} \times_{f_{0}} s$, which proves the result. q.e.d.

We also will need the relative case of Proposition 4.2. For our applications it is convenient to phrase the results in terms of cohomology. We make the following definitions.

Definition 4.5. A map $f: M \rightarrow R$ is said to be orientable if $f^{*} \Lambda^{\max }\left(T^{*} R\right)$ is isomorphic to $\Lambda^{\max }\left(T^{*} M\right)$ and is said to be oriented if such an isomorphism is chosen.

Notice that an oriented map $f: M \rightarrow R$ has a well defined intersection number with oriented submanifolds of the correct dimension. Suppose $S \subset R$ is a smooth oriented manifold so that $\operatorname{dim}(S)+\operatorname{dim}(M)=$ $\operatorname{dim}(R)$ and that $f$ is transverse to $S$. Suppose that $f(m) \in S$. Then we have an exact sequence

$$
0 \rightarrow T^{*} S_{f(m)} \stackrel{\iota^{*}}{\longrightarrow} T^{*} R_{f(m)} \stackrel{f^{*}}{\longrightarrow} T^{*} M_{m} \rightarrow 0 .
$$

This exact sequence induces an isomorphism

$$
\Lambda^{\max }\left(T^{*} M_{m}\right) \otimes f^{*} \Lambda^{\max }\left(T^{*} S\right)_{m}=f^{*} \Lambda^{\max }\left(T^{*} R\right)_{m} .
$$

Using the orientation of $f$ and $S$, we determine whether or not this isomorphism is orientation preserving. This determines the sign of $m$.

Definition 4.6. Let $M$ be an $(n-\delta)$-manifold and let $R$ be a smooth, compact manifold of dimension $n \geq \delta$, possibly with boundary. Let $f: M \rightarrow R$ be an oriented smooth map. We say that a smooth singular cochain $\alpha$ agrees with $(M, f)$ outside an open set $U \subset R$ if for every smooth singular chain $c$ in $C_{\delta}(R \backslash U)$ which is transverse to $f$, the oriented intersection number of $c$ with $f$ equals the Kronecker pairing $\langle\alpha, c\rangle$.

Proposition 4.7. Let $M$ be a smooth $n-\delta$ manifold and let $R$ be a smooth, compact manifold of dimension $n \geq \delta$, possibly with boundary. Suppose that $f: M \rightarrow R$ is an oriented smooth map so that the complement $\overline{f(M)} \backslash(f(M) \cup \partial R)$ is contained in dimension $n-\delta-2$. Then there is a neighborhood $U$ of $\overline{f(M)} \backslash(f(M) \cup \partial R)$ with the following two properties. First, for every neighborhood $V$ of $\overline{f(M)} \backslash(f(M) \cup \partial R)$ with $\bar{V} \subset U$ there is a rational singular smooth 
relative $\delta$-cocycle $\alpha \in C^{\delta}(R)$ which agrees with $(M, f)$ outside $V$. Secondly, any $\delta$-cocycle which agrees with $(M, f)$ outside $U$ defines the same rational cohomology class.

Definition 4.8. We call the cohomology class of the cycle $\alpha$ produced by this lemma $[M, f] \in H^{\delta}(R ; \mathbb{Q})$.

The reader can easily formulate and prove the relative analogue of Proposition 4.4. The next lemma compares the intersection numbers of these classes with the geometric intersection of these maps in the case the domains have complementary dimension.

Lemma 4.9. Let $R$ be a smooth oriented n-manifold, possibly with non-empty boundary. Suppose we are given $r_{+}: M_{+} \rightarrow R$ satisfying the hypotheses of Proposition 4.2 and $r_{-}: M_{-} \rightarrow R$ satisfying the hypotheses of Proposition 4.7. Furthermore suppose that:

(i) $\operatorname{dim}\left(M_{+}\right)+\operatorname{dim}\left(M_{-}\right)=\operatorname{dim}(R)$;

(ii) the image of each map is disjoint from the frontier of the other, i.e.

$$
\overline{r_{+}\left(M_{+}\right)} \cap r_{-}\left(M_{-}\right)=r_{+}\left(M_{+}\right) \cap \overline{r_{-}\left(M_{-}\right)}=r_{+}\left(M_{+}\right) \cap r_{-}\left(M_{-}\right) ;
$$

(iii) $r_{+}$and $r_{-}$are transverse.

Then the signed count of the number of points in the fibered product

$$
M_{+} \times_{\left(r_{+}, r_{-}\right)} M_{-}
$$

is equal to the Kronecker pairing of the classes $\left[M_{+}, r_{+}\right]$and $\left[M_{-}, r_{-}\right]$.

Proof. This is clear from the construction of $\left[M_{ \pm}, r_{ \pm}\right]$. $\quad$ q.e.d.

(ii) Weak limits on cylindrical end manifolds. Given a positive real number $t$, we define $\tau_{t}: \mathbb{R}_{+} \times Y \rightarrow[-t, \infty) \times Y$ to be the translation by $-t$. There is a similarly defined map for bundles over $\mathbb{R}_{+} \times Y$. A multi-set $\mathbf{z} \subset Z$ is an $n$-tuple of points of $Z$ possibly with repetition and where $n$ is an arbitrary non-negative integer. The number of elements of $\mathbf{z}$ is denoted $|\mathbf{z}|$. We recall the Uhlenbeck compactness theorem for cylindrical-end manifolds (Proposition 6.3.1 of [36]), which gives the behavior of weak limits of connections in $M_{\kappa}^{w}(Z)$.

Theorem 4.10. Let $\left[A_{i}\right]$ be a sequence of connections in $M_{\kappa}^{w}(Z)$. After passing to a subsequence we can find the following data:

(a) a finite multi-set $\mathbf{z}^{0} \subset Z$ and finite multi-sets $\mathbf{z}^{1}, \ldots, \mathbf{z}^{N} \subset \mathbb{R} \times Y$;

(b) a bundle $E^{0} \rightarrow Z$ with $c_{1}\left(E^{0}\right)=w$ and a finite-energy $A S D$ connection $A^{0}$ on $E^{0}$; 
(c) bundles $E^{1}, E^{2}, \ldots, E^{N} \rightarrow \mathbb{R} \times Y$ with $c_{1}\left(E^{i}\right)=\left.w\right|_{\mathbb{R} \times Y}$ and connections $A^{j}$ on $E^{j}$ for $j=1, \ldots, N$; if $\mathbf{z}^{j}$ is empty then the connection $A^{j}$ is not equivalent to a product connection;

(d) a finite set of sequences of positive real numbers $\left\{t_{i}^{1}\right\}_{i=1}^{\infty}, \ldots$, $\left\{t_{i}^{N}\right\}_{i=1}^{\infty}$ with

(i) $t_{i}^{1}<t_{i}^{2}<\cdots<t_{i}^{N}$ for all $i$,

(ii) $\lim _{i \mapsto \infty} t_{i}^{j}=\infty$ for all $j=1, \ldots, N$,

(iii) $\lim _{i \mapsto \infty} t_{i}^{j}-t_{i}^{j-1}=\infty$ for $j=2, \ldots, N$.

This data satisfies the following:

$(\alpha)\left[\left.A_{i}\right|_{Z \backslash \mathbf{z}^{0}}\right]$ converges uniformly in the $C^{\infty}$-topology on compact sets of $Z \backslash \mathbf{z}^{0}$ to $\left[\left.A^{0}\right|_{Z \backslash \mathbf{z}^{0}}\right]$ and the curvature densities $\operatorname{tr}\left(F_{A_{i}} \wedge F_{A_{i}}\right)$ converge in the sense of measures on compact subsets of $Z$ to $\operatorname{tr}\left(F_{A^{0}} \wedge F_{A^{0}}\right)+8 \pi^{2} \sum_{z \in \mathbf{z}^{0}} \delta(z) ;$

( $\beta) \quad\left[\left.\tau_{t_{i}^{j}}^{*} A_{i}\right|_{\mathbb{R} \times Y \backslash \mathbf{z}^{j}}\right]$ converges uniformly on compact sets of $(\mathbb{R} \times Y) \backslash \mathbf{z}^{j}$ in the $C^{\infty}$-topology to $\left[\left.A^{j}\right|_{\mathbb{R} \times Y \backslash \mathbf{z}^{j}}\right]$ for $j=1, \ldots, N$, and the curvature densities $\tau_{t_{i}^{*}}^{*} \operatorname{tr}\left(F_{A_{i}} \wedge F_{A_{i}}\right)$ converge in the sense of measures on compact subsets of $\mathbb{R} \times Y$ to $\operatorname{tr}\left(F_{A^{i}} \wedge F_{A^{i}}\right)+8 \pi^{2} \sum_{z \in \mathbf{z}^{i}} \delta(z)$;

$(\gamma)$ the Chern-Weil integral $\kappa=c(A)$ satisfies

$$
\kappa=c\left(A^{0}\right)+\left|\mathbf{z}^{0}\right|+\sum_{j=1}^{N}\left(c\left(A^{j}\right)+\left|\mathbf{z}^{j}\right|\right) ;
$$

( $\delta) \quad r\left(\left[A^{0}\right]\right)=r_{-}\left(\left[A^{1}\right]\right)$, and $r_{+}\left(\left[A^{j}\right]\right)=r_{-}\left(\left[A^{j+1}\right]\right)$ for $1 \leq j<N$.

The second clause of item (c) is to ensure that this collection of limiting data is unique. The kind of limits that arise in this theorem lead naturally to a notion of ideal connection:

Definition 4.11. Any collection of data

$$
\left(\left[A^{0}\right],\left[A^{1}\right], \ldots,\left[A^{N}\right] ; \mathbf{z}^{0}, \mathbf{z}^{1}, \ldots, \mathbf{z}^{N}\right),
$$

as in (a)-(c) satisfying $(\gamma)$ and $(\delta)$, is called an ideal connection with charge $\kappa$.

The integer $N$ is called the length of the connection. The space of ideal connections of length $N$ and charge $\kappa$ can be described as the union of the iterated fibered products

$$
\begin{aligned}
\bar{M}_{\kappa^{0}}^{w}(Z) \times{ }_{\left(r, r_{-}\right)} \bar{M}_{\kappa^{1}}^{w}(\mathbb{R} \times Y) & \times_{\left(r_{+}, r_{-}\right)} \bar{M}_{\kappa^{2}}^{w}(\mathbb{R} \times Y) \ldots \\
& \times_{\left(r_{+}, r_{-}\right)} \bar{M}_{\kappa^{N}}^{w}(\mathbb{R} \times Y)
\end{aligned}
$$


as $\kappa^{0}, \kappa^{1}, \ldots, \kappa^{N} \geq 0$ vary over $\kappa^{0}+\kappa^{1}+\ldots \kappa^{N}=\kappa$. Here $\bar{M}$ denotes the usual Uhlenbeck closure of a moduli space $M$. There are, of course, further constraints on the possible sequences, $\left(\kappa^{0}, \kappa^{1}, \ldots, \kappa^{N}\right)$, that can arise if the fibered product above is non-empty. The following definition encodes the constraint that arises from the Chern-Simons invariants.

Definition 4.13. For a real number $\kappa>0$, we say that a sequence $\kappa^{0}, \kappa^{1}, \ldots, \kappa^{N}$ is $\kappa$-appropriate if the following hold:

(a) $\kappa^{0}, \kappa^{1}, \ldots, \kappa^{N} \geq 0$

(b) $\sum_{i=0}^{N} \kappa^{i} \leq \kappa$;

(c) there is a sequence of flat connections $\rho^{0}, \ldots, \rho^{N}$ on $E^{w} \rightarrow Y$ so that

$$
\text { C.S. }\left(\rho^{0}\right) \equiv \kappa^{0}(\bmod 1)
$$

and

$$
\text { C.S. }\left(\rho^{i}\right)-\text { C.S. }\left(\rho^{i-1}\right) \equiv \kappa^{i}(\bmod 1) \text {, }
$$

for all $i,(1 \leq i \leq N)$.

Note that the definition of $\kappa$-appropriate implicitly uses $Y$ as an oriented manifold. Also, for any $\kappa>0$, there are only finitely many $\kappa$-appropriate sequences. We only consider $\kappa>0$ to avoid problems with flat connections. Using the blow-up trick from [34] this causes no loss of generality on the level of the invariants on $X$ or $X^{o}$ and we will leave the blow-up implicit when dealing with these manifolds, but there are some issues to keep track of on $W^{o}$ so we will be explicit in this case.

The boundary map $r$ on $M_{\kappa}^{w}(Z)$ has a natural extension to ideal connections. If $\left(\left[A^{0}\right], \ldots,\left[A^{N}\right] ; \mathbf{z}^{0}, \ldots, \mathbf{z}^{N}\right)$ is an ideal connection we define

$$
\left(\left[A^{0}\right], \ldots,\left[A^{N}\right] ; \mathbf{z}^{0}, \ldots, \mathbf{z}^{N}\right)=r_{+}\left(\left[A^{N}\right]\right) .
$$

Finally notice that the image of the boundary map can be described in terms of $\vec{T}_{\kappa}$. For example, the image under $r$ of the fibered product (4.12) is contained in

$$
\vec{T}_{\kappa^{N}} \circ \cdots \circ \vec{T}_{\kappa^{1}}\left(r\left(M_{\kappa^{0}}^{w}(Z)\right)\right) .
$$

Consider again a pair $(X, \Sigma)$, with $n=Q(\Sigma)>0$, and the resulting cylindrical-end manifolds $X^{o}$ and $W^{\circ}$, as in the opening paragraph of this section. Fix a line bundle $w$ on $X$, and use the same notation for its restriction to $Y=Y(n, g)$, the boundary of the complement of a 
tubular neighbourhood of $\Sigma$. Because $w$ is given on the whole of $X$, its restriction $w \rightarrow Y$ is identified, up to isotopy, with the pull-back of a bundle from $\Sigma$. We can therefore talk unambiguously about the components $\mathscr{R}_{\mathrm{ev}}^{w}$ etc., of the representation variety $\mathscr{R}^{w}(Y)$. For $\kappa \in \mathbb{R}$, let

$$
\delta(\kappa)=8 \kappa-3\left(1-b^{1}(X)+b^{+}(X)\right) .
$$

If $z \in \mathbb{A}_{\delta}\left(X^{o}\right)$, then the formal dimension of the moduli space

$$
M_{\kappa}^{w}\left(X^{o} ; \mathscr{R}_{\mathrm{ev} / \mathrm{od}}^{w, s}\right) \cap V(z)
$$

is $\delta(\kappa)-\delta$. We can now show that, for $\kappa, g$ and $n$ in a certain range, the frontier of this space under $r$ is contained in dimension $\delta(\kappa)-2$.

Remark. Before stating the results, we remind the reader that the metric on $Y$ (and hence on the ends of $X^{\circ}$ and $W^{\circ}$ ) is fixed, and rather special, because we wish to apply the results of section 3. Accordingly, when we talk about a generic perturbation of the metric on $X^{\circ}$ for example, we are talking about a compactly-supported perturbation. This should be understood throughout this section.

Proposition 4.14. Suppose $X^{\circ}$ is an admissible cylindrical-end 4manifold, arising from the complement $X / \Sigma$ as above. Let $z \in \mathbb{A}_{\delta}(X)$, and suppose that $\delta$ and $\kappa \in \mathbb{R}$ satisfy the following inequalities:

$$
\begin{aligned}
& \delta(\kappa)-\delta \leq 4 g-6 \\
& \delta(\kappa)-\delta \leq 2 g-4-n / 2 \\
& \delta(\kappa)-\delta \leq 2 n-2 .
\end{aligned}
$$

Then for all cylindrical-end metrics on $X^{o}$ in a Baire set, all $\kappa$-appropriate sequences $\kappa^{0}, \kappa^{1}, \ldots, \kappa^{N}$, and all good representatives $V(z) \subset$ $M_{\kappa}^{w}\left(X^{o}\right)$ for $z$, we have

(i) $r\left(M_{\kappa^{0}}^{w}\left(X^{o}\right) \cap V(z)\right)$ is contained in $\mathscr{R}_{\mathrm{ev}}^{w, s} \amalg \mathscr{R}_{\mathrm{od}}^{w, s}$,

(ii) the set

$$
\vec{T}_{\kappa^{N}} \circ \cdots \circ \vec{T}_{\kappa^{1}}\left(r\left(M_{\kappa^{0}}^{w}\left(X^{o} ; \mathscr{R}_{*}^{w, s}\right) \cap V(z)\right)\right)
$$

is contained in $\mathscr{R}_{*}^{w, s}$, for $*=$ 'ev' and 'od',

(iii) the set (4.15) above is contained in dimension $\delta(\kappa)-\delta-2 N$,

(iv) if $S$ is any subset of $\mathscr{R}_{*}^{w, s}$ contained in codimension greater than $\delta(\kappa)-\delta-2 N$, then for a generic metric on $X^{o}$, the set $(4.15)$ is disjoint from $S$. 
Furthermore, for each metric in a generic path of metrics $g_{t}$, items (a) and (b) hold, and

$$
\bigcup_{0 \leq t \leq 1}\left(t, \vec{T}_{\kappa^{N}} \circ \cdots \circ \vec{T}_{\kappa^{1}}\left(r\left(M_{\kappa^{0}}^{w}\left(X^{o} ; \mathscr{R}^{w, s}\right) \cap V(z)\right)\right)\right) \subset[0,1] \times \mathscr{R}_{*}^{w, s}
$$

is contained in dimension $\delta(\kappa)-\delta-2 N+1$, for $*=$ 'ev' and 'od'.

Before giving the proof of this proposition, we deduce the following crucial corollary.

Corollary 4.16. Let $(X, \Sigma)$ be a pair for which $X^{o}$ is admissible. Let $z \in \mathbb{A}_{\delta}\left(X^{o}\right)$, and suppose

$$
\delta(\kappa)-\delta \leq \min \{4 g-6,2 g-4-n / 2,2 n-2\}
$$

Then, for generic cylindrical end metrics $g$ on $X^{o}$ and all good representatives $V(z)$, the set $r\left(M_{\kappa}^{w}\left(X^{o}\right) \cap V(z)\right)$ is contained in a compact subset of $\mathscr{R}_{\mathrm{ev}}^{w, s} \amalg \mathscr{R}_{\mathrm{od}}^{w, s}$, and its frontier is contained in dimension $\delta(\kappa)-\delta-2$.

Proof of Corollary. Without loss of generality, we can assume that $z$ is a monomial $\beta_{1} \beta_{2} \ldots \beta_{r}$ and that $V(z)$ is the transverse intersection of good representatives $V\left(\beta_{i}\right)$ for the $\beta_{i}$. For any subset $I$ of $\{1, \ldots, r\}$, let $z_{I}$ denote the corresponding product and $d_{I}$ its degree.

The set $r\left(M_{\kappa}^{w}\left(X^{o}\right) \cap V(z)\right)$ is contained in $\mathscr{R}_{\mathrm{ev}}^{w, s} \amalg \mathscr{R}_{\mathrm{od}}^{w, s}$ by part $(a)$ of the Proposition above. If $\rho$ lies in the closure of $r\left(M_{\kappa}^{w}\left(X^{o} ; \mathscr{R}_{\mathrm{ev} / \mathrm{od}}^{w, s}\right) \cap\right.$ $V(z)$ ), then the compactness theorem (4.10) and parts $(a)$ and $(b)$ of the Proposition above imply that $\rho$ lies in

$$
\vec{T}_{\kappa^{N}} \circ \cdots \circ \vec{T}_{\kappa^{1}}\left(r\left(M_{\kappa^{0}-s}^{w}\left(X^{o} ; \mathscr{R}_{\mathrm{ev} / \mathrm{od}}^{w, s}\right) \cap V\left(z_{I}\right)\right)\right) \subset \mathscr{R}_{\mathrm{ev} / \mathrm{od}}^{w, s}
$$

for some subset $I \subset\{1, \ldots r\}$ and some $\kappa$-appropriate sequence $\kappa^{0}$, $\kappa^{1}, \ldots, \kappa^{N}$. Furthermore, from the definition of a good representative, the integer $s$ (the number of points of concentration of curvature in $\left.X^{o}\right)$ satisfies

$$
5 s \geq \delta-\delta_{I},
$$

by (2.7). In the case $N=0$, the dimension of the moduli space

$$
M_{\kappa^{\circ}-s}^{w} \kappa\left(X^{o} ; \mathscr{R}_{\mathrm{ev} / \mathrm{od}}^{w, s}\right) \cap V\left(z_{I}\right)
$$


is therefore at most $\delta(\kappa)-\delta-3 s$, and its image under $r$ is therefore certainly contained in dimension $\delta(\kappa)-\delta-3$ if $s$ is non-zero. If $N \neq 0$, then by part $(c)$ of the above Proposition, the set (4.17) is contained in dimension

$$
\delta(\kappa)-8 s-\delta_{I}-2 N \geq \delta(\kappa)-\delta-2 N .
$$

If $\rho$ is actually in the frontier rather than just the closure, then at least one of $N$ and $s$ must be non-zero. There are only finitely many $\kappa$-appropriate sequences, so the conclusion is that $\rho$ must lie in one a finite union of subsets of $\mathscr{R}_{\mathrm{ev} / \mathrm{od}}^{w, s}$, each of which is contained in dimension at most $\delta(\kappa)-\delta-2$. q.e.d.

Proof of 4.14. We give the proof when there are no homology classes; that is, when $z=1$ and $\delta=0$. The general case is essentially the same. Since $X^{\circ}$ is admissible, Lemma 2.14 tells us that twisted reducible connections cause no problem for a generic metric on $X^{o}$.

First we prove (a). As a function of $m$, the formal dimension of the moduli spaces $M_{\kappa}^{w}\left(X^{o} ; \mathscr{R}_{m}\right)$,

$$
\delta(\kappa)-8 \frac{m^{2}}{n}+4 m+3-2 g,
$$

achieves its maximum value, $\delta(\kappa)+n / 2+3-2 g$, when $m=n / 4$. Thus inequality (ii) implies that this formal dimension is negative, and hence this moduli space is empty for generic cylindrical-end metric on $X^{o}$. (See also [27], Lemma 3.8.) To analyze $M_{\kappa}^{w}\left(X^{o} ; \mathscr{R}_{0}^{w}\right)$, we use the local structure theorem for cylindrical end manifolds Theorem 14.0.4 of [36] (see Theorem 2.13). For a generic metric on $X^{o}$ the dimension of the preimage under $r$ of the ad-trivial stratum of $\mathscr{R}_{\mathrm{ev}}^{w}$ or $\mathscr{R}_{\mathrm{od}}^{w}$ is

$$
8 \kappa-3\left(1-b^{1}(\hat{Z})+b_{+}^{2}(\hat{Z})\right)-6 g+3=\delta(\kappa)-6 g+3,
$$

while the preimage of the $S^{1}$-reducible stratum has dimension

$$
8 \kappa-3\left(1-b^{1}(\hat{Z})+b_{+}^{2}(\hat{Z})\right)-4 g+2=\delta(\kappa)-4 g+5 .
$$

Thus, if inequality (i) is satisfied, these dimensions are negative, and hence for a generic metric these moduli spaces are empty.

Now we prove (b), (c), and (d) together by induction on $N$. For $N=0$, there is nothing to prove in (b), while (c) and (d) are the 
dimension formula and generic metrics theorem (2.13). So suppose $N>0$, and that (b), (c), and (d) hold with $N-1$ in place of $N$. There are two cases to consider, corresponding to the even and odd components. We treat only the former, because the two are otherwise identical. Consider a $\kappa$-appropriate sequence $\kappa^{0}, \kappa^{1}, \ldots, \kappa^{N}$ and the set

$$
\vec{T}_{\kappa^{N}} \circ \cdots \circ \vec{T}_{\kappa^{1}}\left(r\left(M_{\kappa^{0}}^{w}\left(X^{o}\right)\right) \cap \mathscr{R}_{\mathrm{ev}}^{w, s}\right) .
$$

By the induction hypothesis, this has the form

$$
\vec{T}_{\kappa^{N}}(K)
$$

for some subset $K \subset \mathscr{R}_{\mathrm{ev}}^{w, s}$ contained in dimension $\delta(\kappa)-8 \kappa^{N}-2 N+$ 2. By part (d), we can assume that $K$ is disjoint from any set $S$ contained in codimension larger than this, provided that $S$ is specified independently of the metric on the interior of $X^{o}$.

Inequality (iii) implies $\delta\left(\kappa^{0}\right)+8\left(\kappa^{1}+\cdots+\kappa^{N}\right) \leq \delta(\kappa) \leq 2 n-2$. For a generic metric on $X^{o}$, we must have $d\left(\kappa^{0}\right) \geq 0$ and hence $\kappa^{i}<n / 4$ for all $i$, and for $i=N$ in particular. Thus, the hypotheses of Propositions 3.2, $3.4,3.5,3.6$ and corollary 3.3 are satisfied when we consider $\vec{T}_{\kappa^{N}}(K)$.

Let us now prove (b). Proposition 3.4 immediately implies that

$$
\vec{T}_{\kappa^{N}}(K) \cap \mathscr{R}_{\mathrm{od}}^{w}
$$

is empty. Suppose that

$$
\vec{T}_{\kappa^{N}}(K) \cap \mathscr{R}_{m}^{w}
$$

Then $K$ meets the set $S_{m}=\stackrel{\leftarrow}{T}\left(\mathscr{R}_{m}\right) \cap \mathscr{R}_{\mathrm{ev}}^{w, s}$. By Proposition 3.6, $\left(\mathscr{R}_{m}\right) \cap \mathscr{R}_{\mathrm{ev}}^{w, s}$ is empty unless $\kappa^{N} \geq m^{2} / n$ and is contained in dimension

$$
4 g-4+4 m+6\left(\kappa^{N}-m^{2} / n\right) \leq 4 g-4+4 m+8\left(\kappa^{N}-m^{2} / n\right)
$$

otherwise. This achieves its maximum when $m=n / 4$, so $S_{m}$ is contained in codimension

$$
2 g-2-\frac{n}{2}-8 \kappa^{N}
$$

By inequality (ii), this is strictly greater than $\delta(\kappa)-8 \kappa^{N}$, which in turn is at least as large as the dimension of $K$. By the induction hypothesis 
on part (d), we may assume that $K$ does not meet $S_{m}$, so obtaining a contradiction. A very similar argument shows that

$$
\vec{T}_{\kappa^{N}}(K) \cap \mathscr{R}_{\mathrm{ev}}^{w, s i n g}
$$

is empty, using the estimate on the dimension of $\overleftarrow{T}_{\kappa^{N}}\left(\mathscr{R}_{\mathrm{ev}}^{w, s i n g}\right)$ provided by Proposition 3.5. This competes the induction step for (b).

The induction step for (c) follows from Proposition 3.2, which says that $\vec{T}_{\kappa^{N}}(K) \cap \mathscr{R}_{\mathrm{ev}}^{w, s}$ is contained in dimension $\operatorname{dim}(K)+6 \kappa^{N}$. In this situation $\kappa^{N}$ is a positive integer, and so $6 \kappa^{N} \leq 8 \kappa^{N}-2$, which we can combine with the induction hypothesis to give

$$
\begin{aligned}
\operatorname{dim}(K)+6 \kappa^{N} & \leq\left(\delta(\kappa)-8 \kappa^{N}-2(N-1)\right)+8 \kappa^{N}-2 \\
& \leq \delta(\kappa)-2 N
\end{aligned}
$$

as required. To prove (d), consider a subset $S$ of $\mathscr{R}_{\mathrm{ev}}^{w, s}$, and let $S^{\prime}$ be $\overleftarrow{T}_{\kappa^{N}}(S) \subset \mathscr{R}_{\mathrm{ev}}^{w, s}$. If $S$ is contained in codimension greater than $\delta(\kappa)-2 N$, then by Proposition $3.2, S^{\prime}$ is contained in codimension greater than $\delta(\kappa)-8 \kappa^{N}+2$. By the induction hypothesis, $S^{\prime}$ will be disjoint from $K$ for a generic metric, so $S$ will be disjoint from $\vec{T}_{\kappa^{N}}(K)$ as required. This completes the proof of (b), (c), and (d).

The result for a generic path of metrics is proved similarly. q.e.d.

Next, we consider the cylindrical end manifold $W^{o}$. In order to avoid problems arising from flat connections, we consider the blowup $W^{o} \# \overline{\mathbb{C P}}^{2}$ and take $U(2)$-bundle with first Chern class $\bar{w}=w+E$ where $E$ is the cohomology class Poincaré dual to the exceptional curve $e$. Given any $\kappa$, we use the notation $\bar{\kappa}$ for

$$
\bar{\kappa}=\kappa+\frac{1}{4}
$$

so as to hide the effect of the blow-up. We will always consider metrics on $W^{o} \# \overline{\mathbb{C P}}^{2}$ obtained via the connected sum from metrics on $W^{o}$ and $\overline{\mathbb{C P}}^{2}$ in a standard fashion, for example as in Chapter 7 of [11]. Let $R$ be a parameter controlling the neck length. We call a metric corresponding to a value of the parameter $R$, a metric with neck of length $R$. The following lemma follows from a straightforward compactness argument.

Lemma 4.18. For all $\kappa \in \mathbb{R}$ and all compact sets $\mathcal{K}_{1}, \mathcal{K}_{2}$ of metrics on $X$ and $\overline{\mathbb{C P}}^{2}$, there is an $R=R\left(\kappa, \mathcal{K}_{1}, \mathcal{K}_{2}\right)>0$ so that for any $\kappa^{\prime} \leq \kappa$ and any metric on the connected sum, formed from metrics 
belonging to $\mathcal{K}_{i}$, joined with a tube of length $R^{\prime} \geq R$, the moduli spaces $M_{\bar{\kappa}^{\prime}}^{\bar{w}}\left(W^{o} \# \overline{C P}^{2} ; \mathscr{R}_{m}^{\bar{w}}\right)$ contain no reducible connections.

The proposition below parallels Proposition 4.14. Note that although $W^{o}$ has a cylindrical end modelled on $\bar{Y}$, we identify $\mathscr{R}^{w}(\bar{Y})$ with $\mathscr{R}^{w}(Y)$, and label its components accordingly. So $Y$ is still the manifold $Y(n, g)$, with $n$ positive.

Proposition 4.19. Let $W^{o}$ be as above, and let $\gamma \in \mathbb{A}_{\delta}\left(W^{o}\right)$. Suppose $d$ and $\kappa$ satisfy the following inequalities:

$$
\begin{aligned}
& \delta \leq 4 g-6, \\
& \delta \leq 2 g-4-n / 2, \\
& \kappa<\frac{1}{4} n .
\end{aligned}
$$

Then for all cylindrical-end metrics on $W^{\circ} \# \overline{\mathbb{C P}}^{2}$ in a Baire set of metrics with neck of length at least $R(\kappa)$, for all $\kappa$-appropriate sequences $\kappa^{0}, \kappa^{1}, \ldots, \kappa^{N}$ with length $N>0$ and all good representatives $V(e \gamma)$ for $e \gamma$, the set

$$
\overleftarrow{T}_{\kappa^{N}} \circ \cdots \circ \overleftarrow{T}_{\kappa^{1}}\left(r\left(M_{\bar{\kappa}^{0}}^{\bar{w}}\left(W^{o} \# \overline{\mathbb{C P}}^{2}\right) \cap V(e \gamma)\right)\right) \cap \mathscr{R}_{\mathrm{ev} / \mathrm{od}}^{w, s}
$$

is contained in dimension $6 g-6-\delta+8 \kappa-2$. Furthermore, for a generic one-parameter family of metrics with neck of length at least $R(\kappa)$, we have that

$$
\begin{gathered}
\bigcup_{0 \leq t \leq 1}\left(t, \overleftarrow{T}_{\kappa^{N}} \circ \cdots \circ{\stackrel{\leftarrow}{\kappa^{1}}}\left(r\left(M_{\bar{\kappa}^{0}}^{\bar{w}}\left(W^{o} \# \overline{\mathbb{C P}}^{2}\right) \cap V(e \gamma)\right)\right) \cap \mathscr{R}_{\mathrm{ev} / \mathrm{od}}^{w, s}\right) \\
\subset[0,1] \times \mathscr{R}_{\mathrm{ev} / \mathrm{od}}^{w, s}
\end{gathered}
$$

is contained in dimension $6 g-6-\delta+8 \kappa-1$.

There is the following Corollary which can be deduced from the above proposition just as (4.16) was deduced from Proposition 4.14. Note that $6 g-6-\delta+8 \kappa$ is the formal dimension of the moduli space

$$
M_{\bar{\kappa}}^{\bar{w}}\left(W^{o} \# \overline{\mathbb{C P}}^{2} ; \mathscr{R}_{\text {ev } / \text { od }}^{w, s}\right) \cap V(e \gamma) .
$$

Corollary 4.21. Let $\gamma \in \mathbb{A}_{d}\left(W^{o}\right)$. Suppose that

$$
\delta \leq \min \{4 g-6,2 g-4-n / 2\}
$$

and $\kappa<\frac{1}{4} n$. Then for a generic cylindrical-end metric on $W^{\circ} \# \overline{\mathbb{C P}}^{2}$ and generic good representatives $V(e \gamma)$ for $e \gamma$, the frontier of

$$
r: M_{\bar{\kappa}}^{\bar{w}}\left(W^{o} \# \overline{C P}^{2} ; \mathscr{R}_{\text {ev/od }}^{w, s}\right) \cap V(e \gamma) \rightarrow \mathscr{R}_{\text {ev/od }}^{w, s}
$$


is contained in dimension $6 g-6-\delta+8 \kappa-2$.

Proof of 4.19. First, we need to consider the generic metrics theorem in relation to moduli spaces of the form

$$
M_{\bar{\kappa}^{\prime}}^{\bar{w}}\left(W^{o} \# \overline{\mathbb{C P}}^{2} ; \mathscr{R}_{\mathrm{ev} / \mathrm{od}}^{w, s}\right) \cap V(e \gamma) .
$$

There are no reducibles in this moduli space, because the boundary values are in the irreducible part of the representation variety $\mathscr{R}_{\mathrm{ev} / \mathrm{od}}^{w, s}$. We must also consider twisted reducibles. For any non-trivial real line bundle $\lambda$, we have $b^{+}(\lambda)=0$ and $b^{1}(\lambda)=2 g-2$. To see this, observe first that any non-trivial two-fold cover of $W^{\circ} \# \overline{\mathbb{C P}}^{2}$ is diffeomorphic to a tubular neighborhood of a surface of positive square connect-summed with two copies $\overline{\mathbb{C P}}^{2}$, and hence the intersection from on the anti-invariant part of the two-dimensional homology has $b^{-}=1$ and $b^{+}=0$, so $b^{+}(\lambda)=0$. The first cohomology, $H^{1}$ of the two-fold cover, has rank $4 g-2$ and the invariant part of $H^{1}$ has rank $2 g$, hence $b^{1}(\lambda)=2 g-2$ as claimed. Since the smallest formal dimension of a non-empty moduli space is $6 g-4>2 g-2$, Lemma 2.4 applies, and we deduce that for a generic metric the twisted reducibles are smooth points of the above moduli space. After cutting down, it is therefore a smooth manifold of dimension $6 g-6+8 \kappa^{\prime}-\delta$.

The dimension counting argument is now as follows. There are, as usual, two cases, corresponding respectively to the even and odd components; we shall talk only about $\mathscr{R}_{\mathrm{ev}}^{w, s}$. If $\kappa^{0}, \kappa^{1}, \ldots, \kappa^{N}$ is a $\kappa$ appropriate sequence, then inequality (iii) implies that all the $\kappa^{i}$ are less than $\frac{1}{4} n$ so that the hypotheses of Propositions 3.2, 3.4, 3.5, 3.6, and Corollary 3.3 are satisfied for all the moduli spaces on the cylinder that we encounter. In particular, by Proposition 3.4, we will not encounter any ASD connection asymptotic to the odd component $\mathscr{R}_{\text {od }}^{w}$.

Consider first the subset of (4.20) defined as

$$
\begin{aligned}
\mathscr{R}_{\mathrm{ev}}^{w, s} \cap \overleftarrow{T}_{\kappa^{N}}\left(\mathscr { R } _ { \mathrm { ev } } ^ { w , s } \cap \overleftarrow { T } _ { \kappa ^ { N - 1 } } \cdots \cdots \left(\mathscr{R}_{\mathrm{ev}}^{w, s} \cap \overleftarrow{T}_{\kappa^{1}}\right.\right. \\
\left.\left.\times \quad\left(r\left(M_{\bar{\kappa}^{0}}^{\bar{w}}\left(W^{o} \# \overline{\mathbb{C P}}^{2} ; \mathscr{R}_{\mathrm{ev}}^{w, s}\right) \cap V(e \gamma)\right)\right)\right) \cdots\right)
\end{aligned}
$$

(the set arising from sequences of connections on the cylinder all of whose end-limits belong to $\mathscr{R}_{\mathrm{ev}}^{w, s}$ ). The estimate for the dimension of this follows immediately from the generic metrics theorem applied to the moduli space on $W^{\circ} \# \overline{C P}^{2}$ and repeated application of Proposition 3.2 . 
Every point of (4.20) which is not contained in (4.22) is contained in

$$
\mathscr{R}_{\mathrm{ev}}^{w, s} \cap \overleftarrow{T}_{\kappa^{N}}\left(\mathscr{R}_{\mathrm{ev}}^{w, s} \cap \overleftarrow{T}_{\kappa^{N-1}}\left(\cdots\left(\mathscr{R}_{\mathrm{ev}}^{w, s} \cap \overleftarrow{T}_{\kappa^{i}}(\mathcal{S})\right) \cdots\right)\right)
$$

where $\mathcal{S}$ is one of the components $\mathscr{R}_{m}^{w}$ or $\mathscr{R}_{\mathrm{ev}}^{w, s i n g}$ and $1 \leq i<N$. If $\mathcal{S}$ is $\mathscr{R}_{m}^{w}$, then by 3.4 and arguing as before, either this last set is empty if $\kappa^{i}<m^{2} / n$, or it is contained in dimension

$$
\begin{aligned}
6\left(\sum_{j=i+1}^{N} \kappa^{j}\right)+4 g-4+4 & +6\left(\kappa^{i}-m^{2} / n\right) \\
\leq & 6\left(\sum_{j=i}^{N} \kappa^{j}\right)+4 g-4+\frac{1}{2} n \\
& <8 \kappa+6 g-6-\left(2 g-2-\frac{1}{2} n\right) \\
& <8 \kappa+6 g-6-(\delta+2)
\end{aligned}
$$

by inequality (ii). If $\mathcal{S}$ is $\mathscr{R}_{\mathrm{ev}}^{w, s i n g}$, then by proposition 3.5 the set is contained in dimension

$$
6\left(\sum_{j=i+1}^{N} \kappa^{j}\right)+2 g-2+6 \kappa^{i}<8 \kappa-6 g-6-\delta-2,
$$

where we used inequality (i) to make the estimate. In all cases then, the possible locus for a point of (4.20) satisfies the required dimension estimate. We again omit the similar argument which deals with a oneparameter family of metrics. q.e.d.

The last Proposition and Corollary in this subsection are a pair similar to the two results above, but they deal with connections on $W^{o}$ which are singular along the embedded surface $\Sigma \subset W^{o}$. In considering the moduli spaces of singular connections

$$
M_{\kappa}^{w, p, \alpha}\left(W^{o}, \Sigma ; \mathscr{R}^{w}(Y)\right),
$$

we shall always be dealing with $\alpha$ close to zero. Note that if $\alpha<1 /(2 n)$, then there can be no flat connections in this moduli space (so we will not need to blow up the manifold at a point). There are no nonflat reducibles because $b^{-}=0$ and there are no twisted reducibles because $H_{1}(\Sigma ; \mathbb{Z} / 2)$ maps onto $H_{1}\left(W^{o} ; \mathbb{Z} / 2\right)$ (see the remarks before Lemma 2.22). Finally, recall that the metric on $W^{o}$ will have a coneangle $2 \pi / \nu$ normal to $\Sigma$ whenever we deal with singular connections.

Proposition 4.23. Let $p \in \mathbb{Z}$ satisfy the parity condition (2.20), and fix $\delta \in \mathbb{N}, \kappa \in \mathbb{R}_{+}$and $\alpha \in\left(0, \frac{1}{2}\right)$. Suppose these satisfy the inequalities

$$
\delta+2 p \leq 4 g-6,
$$


(ii)

(v)

$$
\alpha((g-1)-p)-\alpha^{2} n \leq 1 /(4 n) .
$$

Fix $\gamma \in \mathbb{A}_{\delta}\left(W^{o} \backslash \Sigma\right)$. Then for all sufficiently small cone-angles, all cylindrical-end metrics on $W^{o}$ in a Baire set, all $\kappa$-appropriate sequences $\kappa^{0}, \ldots, \kappa^{N}$ of length $N>0$ and all good representatives $V(\gamma)$ for $\gamma$, the set

$$
\overleftarrow{T}_{\kappa^{N}} \circ \cdots \circ \overleftarrow{T}_{\kappa^{1}}\left(r\left(M_{\kappa^{0}}^{w, p, \alpha}\left(W^{o}, \Sigma\right) \cap V(\gamma)\right)\right) \cap \mathscr{R}_{\mathrm{ev} / \mathrm{od}}^{w, s}
$$

is contained in dimension $6 g-6+8 \kappa-\delta-2 p-2$. Furthermore, for a generic one-parameter family of metrics, the set

$$
\begin{gathered}
\bigcup_{0 \leq t \leq 1}\left(t, \overleftarrow{T}_{\kappa^{N}} \circ \cdots \circ \stackrel{\leftarrow}{T}_{\kappa^{1}}\left(r\left(M_{\kappa^{0}}^{w, p, \alpha}\left(W^{o}, \Sigma\right) \cap V(\gamma)\right)\right) \cap \mathscr{R}_{\mathrm{ev} / \mathrm{od}}^{w, s}\right) \\
\subset[0,1] \times \mathscr{R}_{\mathrm{ev} / \mathrm{od}}^{w, s}
\end{gathered}
$$

is contained in dimension $6 g-6+8 \kappa-\delta-2 p-1$.

Proof. The inequality (iv) excludes the possibility of flat reducible connections, as stated above. The remaining dimension-counting argument is otherwise much the same in the proof of Proposition 4.19, once we make the following observation. If $\kappa^{0}, \kappa^{1}, \ldots, \kappa^{N}$ is a $\kappa$ appropriate sequence coming from an ideal connection corresponding to a limit point of $M_{\kappa}^{w, p, \alpha}\left(W^{o}, \Sigma\right)$, then consideration of the ChernSimons invariants implies for all $i>0$ we have $\kappa^{i} \geq 1 /(4 n)$, because (2.11) shows that the Chern-Simons invariants are rational with denominator $4 n$ (recall that $m$ may be a half-integer). The Chern-Weil formula (2.16) says that the total action of such an ideal connection is

$$
\kappa+\alpha((g-1)-p)-\alpha^{2} n .
$$

Inequality (v) tells us that this is less than $\kappa+1 /(4 n)$. Thus the same $\kappa$-appropriate sequences can arise as in the proof of Proposition 4.19. q.e.d.

Corollary 4.24. Fix $p_{0} \in \mathbb{Z}$. Take $p \geq p_{0}$ and $\gamma \in \mathbb{A}_{\delta}\left(W^{o} \backslash \Sigma\right)$. Suppose

$$
\delta+2 p \leq \min \{4 g-6,2 g-4-n / 2\}
$$


and choose $\alpha<1 /(2 n)$, also satisfying the inequality

$$
\alpha\left((g-1)-p_{0}+n / 2\right)-\alpha^{2} n<1 /(4 n) .
$$

Then for all cylindrical-end metrics on $W^{\circ}$ in a Baire set, and all good representatives $V(\gamma)$ for $\gamma$, the frontier of

$$
r: M_{\kappa^{0}}^{w, p, \alpha}\left(W^{o}, \Sigma ; \mathscr{R}_{\mathrm{ev} / \mathrm{od}}^{w, s}\right) \cap V(\gamma) \rightarrow \mathscr{R}_{\mathrm{ev} / \mathrm{od}}^{w, s}
$$

is contained in dimension $6 g-6+8 \kappa-\delta-2 p-2$.

Proof. The Uhlenbeck closure of the moduli space $M_{\kappa}^{w, p, \alpha}\left(W^{o}, \Sigma\right)$ involves moduli spaces $M_{\kappa^{\prime}}^{w, p^{\prime}, \alpha}$, where $p^{\prime}$ may be different from $p$. Choose the cone angle $2 \pi / \nu$ for the metric along $\Sigma$ so small that, for the given value of $\alpha$, Proposition 2.12 of [27] holds. This proposition implies that the change in instanton and monopole numbers, $(-\Delta \kappa,-\Delta l)$, satisfy the constraints

$$
\Delta \kappa \geq 0 \quad \text { and } \quad \Delta \kappa+\Delta l \geq 0
$$

Thus if $l^{\prime}=\frac{1}{2}\left((g-1)-p^{\prime}\right)$, then $l^{\prime} \leq l+\kappa$, and this translates into the inequality

$$
\frac{1}{2}\left((g-1)-p^{\prime}\right) \leq \frac{1}{2}((g-1)-p)+\kappa .
$$

Since $p \geq p_{0}$ and $\kappa<n / 4$, we obtain $(g-1)-p^{\prime} \leq(g-1)-p_{0}+n / 2$. So the second inequality on $\alpha$ in the statement of the Corollary ensures that condition ( $\mathrm{v}$ ) of Proposition 4.23 holds for $p^{\prime}$. As in the proof of Proposition 4.23 , this condition ensures that the same $\kappa$-appropriate sequences arise here as in the non-singular case, and the dimensioncounting proceeds as before. q.e.d.

(iii) Invariants for manifolds with boundary. The work of the previous two subsections allows us to define relative invariants the manifolds $W^{o}$ and $X^{\circ}$ taking values in a truncated version of the Floer homology of $Y$. We fix $n$ and $g$, with $n>0$, and let $Y=Y(n, g)$. Let $w$ be a line-bundle on $Y$, given as the pull-back of a line bundle on $\Sigma$, so that we can distinguish the components $\mathscr{R}_{\mathrm{ev}}^{w}, \mathscr{R}_{\mathrm{od}}^{w}$ and $\mathscr{R}_{m}^{w}$ of its representation variety. Set

$$
\begin{aligned}
\Delta & =\min \{4 g-6,2 g-4-(n / 2), 2 n-2\} \\
& =\min \{2 g-4-(n / 2), 2 n-2\},
\end{aligned}
$$


and define the Floer homology and cohomology of $Y$ as the ordinary homology and cohomology of the irreducible parts of $\mathscr{R}^{w}(Y)$, truncated at degree $\Delta$ :

Definition 4.27. The (truncated) Floer homology group of $Y$ is the sum

$$
F^{w}(Y)=\bigoplus_{0 \leq i \leq \Delta} H_{i}\left(\mathscr{R}_{\mathrm{ev}}^{w, s}\right) \oplus H_{i}\left(\mathscr{R}_{\mathrm{od}}^{w, s}\right) .
$$

The truncated Floer cohomology group of $Y$ is the sum

$$
\bar{F}^{w}(Y)=\bigoplus_{0 \leq i \leq \Delta} H^{i}\left(\mathscr{R}_{\mathrm{ev}}^{w, s}\right) \oplus H^{i}\left(\mathscr{R}_{\mathrm{od}}^{w, s}\right) .
$$

We usually understand that the coefficients are real or rational.

Sometimes the mod 8 grading of $F^{w}(Y)$ and $\bar{F}^{w}(Y)$ is of significance; it arises from the mod 8 reduction of the ordinary grading on the homology and cohomology of the representation variety. There is a natural bilinear pairing

$$
\langle\cdot, \cdot\rangle: F^{w}(Y) \times \bar{F}^{w}(Y) \rightarrow \mathbb{Q} \text { or } \mathbb{R}
$$

which is the sum of the usual pairings between the ordinary homology and cohomology in each degree $i$. The Floer homology and cohomology also have a decomposition coming from the decomposition of the irreducible representation variety into its two components. We set

$$
\begin{aligned}
& F_{\mathrm{ev}}^{w}(Y)=\bigoplus_{0 \leq i \leq \Delta} H_{i}\left(\mathscr{R}_{\mathrm{ev}}^{w, s}\right) \\
& F_{\mathrm{od}}^{w}(Y)=\bigoplus_{0 \leq i \leq \Delta} H_{i}\left(\mathscr{R}_{\mathrm{od}}^{w, s}\right)
\end{aligned}
$$

with analogous definitions for $\bar{F}^{w}$. These decompositions should not be confused with the other $\mathbb{Z} / 2$ grading, arising from the decomposition of $\oplus H_{i}\left(\mathscr{R}^{w}\right)$ according to the parity of $i$. Finally, there is a natural filtration of $F^{w}(Y)$ and $\bar{F}^{w}(Y)$. For any $\delta \leq \Delta$ we write

$$
\begin{aligned}
& F^{w}(Y)_{(\delta)}=\bigoplus_{0 \leq i \leq \delta} H_{i}\left(\mathscr{R}_{\mathrm{ev}}^{w, s}\right) \oplus H_{i}\left(\mathscr{R}_{\mathrm{od}}^{w, s}\right), \\
& \bar{F}^{w}(Y)^{(\delta)}=\bigoplus_{0 \leq i \leq \delta} H^{i}\left(\mathscr{R}_{\mathrm{ev}}^{w, s}\right) \oplus H^{i}\left(\mathscr{R}_{\mathrm{od}}^{w, s}\right) .
\end{aligned}
$$


The chosen value of $\Delta$ for the truncation arises from the inequalities in the previous subsection (it is the right-hand side in Corollary 4.16), and is not meant to be best possible.

Now let $(X, \Sigma)$ be again an admissible pair, and let $X^{o}$ and $W^{\circ}$ be the corresponding cylindrical-end manifolds. Suppose that $X^{o}$ is also admissible in the sense of (2.14). Let $w \rightarrow X$ be any line bundle, and let $w$ also stand for the restriction of this line bundle to $X^{o}, W^{o}$ and $Y(n, g)=\partial X \backslash N(\Sigma)$. Note that on the end of $W^{o}$, the line bundle naturally acquires the structure of a cylindrical-end bundle. We continue to suppose the self-intersection number $n$ is positive. We introduce the abbreviation $\mathbb{A}(Z)_{(\Delta)}$ for the truncation of $\mathbb{A}(Z)$ :

$$
\mathbb{A}(Z)_{(\Delta)}=\{\gamma \in \mathbb{A}(Z) \mid \operatorname{deg}(\gamma) \leq \Delta\} .
$$

We use the notation $\imath$ and $\jmath$ for the inclusions

$$
\begin{aligned}
& \imath: W^{o} \simeq \Sigma \hookrightarrow X \\
& \jmath: X^{o} \simeq X / \Sigma \hookrightarrow X,
\end{aligned}
$$

and $\imath_{*}, \jmath_{*}$ for the induced maps in homology. The main result of this section is the following theorem.

Theorem 4.28. Let $X^{o}, W^{o}$ and $w$ be as above. Let $p$ be a nonnegative integer satisfying the parity condition (2.20), with $0 \leq 2 p \leq \Delta$. There are linear maps

$$
\begin{array}{rlll}
D_{X^{\circ}}^{w} & : \mathbb{A}\left(X^{o}\right) & \longrightarrow F^{w}(Y), \\
D_{W^{\circ}}^{w} & : \mathbb{A}\left(W^{o}\right)_{(\Delta)} & \longrightarrow \bar{F}^{w}(Y), \\
D_{W^{o}, \Sigma}^{w, p} & : \mathbb{A}\left(W^{o} \backslash \Sigma\right)_{(\Delta-2 p)} & \longrightarrow \bar{F}^{w}(Y)
\end{array}
$$

so that for all $\gamma \in \mathbb{A}\left(W^{o}\right)_{(\Delta)}$ and $z \in \mathbb{A}\left(X^{o}\right)$ we have

$$
D_{X}^{w}\left(\imath_{*}(\gamma) \jmath_{*}(z)\right)=\left\langle D_{W^{\circ}}^{w}(\gamma), D_{X^{\circ}}^{w}(z)\right\rangle,
$$

while for $\gamma \in \mathbb{A}\left(W^{o} \backslash \Sigma\right)_{(\Delta-2 p)}$ we have

$$
D_{X, \Sigma}^{w, p}\left(\imath_{*}(\gamma) \jmath_{*}(z)\right)=\left\langle D_{W^{o}, \Sigma}^{w, p}(\gamma), D_{X^{\circ}}^{w}(z)\right\rangle .
$$

All three of the linear maps are natural with respect to diffeomorphisms, with the single exception of $D_{X^{\circ}}^{w}$ in the case that $b^{+}(X)=2$, in which 
case the invariant may depend (a priori) on a choice of generic Riemannian metric. The maps $D_{W^{\circ}}^{w}$ and $D_{W^{\circ}, \Sigma}^{w, p}$ respect the filtration by degree, in that for any $\delta \leq \Delta$ we have

$$
\begin{aligned}
D_{W^{\circ}}^{w} & : \mathbb{A}\left(W^{o}\right)_{(\delta)} \rightarrow \quad \bar{F}^{w}(Y)^{(\delta)} \\
D_{W^{\circ}, \Sigma}^{w, p} & : \mathbb{A}\left(W^{o} \backslash \Sigma\right)_{(\delta-2 p)} \rightarrow \bar{F}^{w}(Y)^{(\delta)} .
\end{aligned}
$$

Remark. We will see in section 5 (Corollary 5.8) that, in fact, the invariant $D_{X^{\circ}}^{w}$ is independent of metric, even in the case $b^{+}(X)=2$.

Proof of the Theorem. We begin with the construction of the maps, starting with the case of $X^{o}$. Fix $z \in \mathbb{A}_{d}\left(X^{o}\right)$, which we may suppose is a monomial, expressed as a product of integral homology classes. Pick a good representative $V(z) \subset M_{\kappa}^{w}\left(X^{o} ; \mathscr{R}_{\mathrm{ev}}^{w, s} \amalg \mathscr{R}_{\mathrm{od}}^{w, s}\right)$ and consider the cut-down moduli space with its end-limit map $r$ :

$$
r: M_{\kappa}^{w}\left(X^{o} ; \mathscr{R}_{\mathrm{ev}}^{w, s} \amalg \mathscr{R}_{\mathrm{od}}^{w, s}\right) \cap V(z) \rightarrow \mathscr{R}_{\mathrm{ev}}^{w, s} \amalg \mathscr{R}_{\mathrm{od}}^{w, s} .
$$

The domain of $r$ is a smooth oriented manifold of dimension $\delta(\kappa)-\delta$. If $\delta(\kappa)-\delta \leq \Delta$, then Corollary 4.16 implies that for a generic metric and generic good representative $V(z)$, the image of $r$ is contained in a compact subset of the irreducible representation variety, and its frontier is contained in dimension $\delta(\kappa)-\delta-2$. We can choose a compact subset containing the image of $r$,

$$
\mathscr{R}^{\sim} \subset \mathscr{R}_{\mathrm{ev}}^{w, s} \amalg \mathscr{R}_{\mathrm{od}}^{w, s}
$$

which has the same dimension $6 g-6$, is a manifold with smooth boundary, and is a deformation retract of the whole. (Take the complement of a regular neighbourhood of the singular stratum in $\mathscr{R}_{\mathrm{ev}}^{w} \amalg \mathscr{R}_{\mathrm{od}}^{w}$.) Then by Proposition 4.2, for all such $\kappa$ satisfying the above inequality, the map $r$ defines a rational homology class

$$
\left[M_{\kappa}^{w}\left(X^{o} ; \mathscr{R}_{\mathrm{ev}}^{w, s} \amalg \mathscr{R}_{\mathrm{od}}^{w, s}\right) \cap V(z), r\right] \in H_{\delta(\kappa)-\delta}\left(\mathscr{R}_{\mathrm{ev}}^{w, s}\right) \oplus H_{\delta(\kappa)-\delta}\left(\mathscr{R}_{\mathrm{od}}^{w, s}\right) .
$$

We set

$$
D_{X^{\circ}}^{w}(z)=\sum_{\substack{\kappa \\ \delta(\kappa)-\delta \leq \Delta}}\left[M_{\kappa}^{w}\left(X^{o} ; \mathscr{R}_{\mathrm{ev}}^{w, s} \amalg \mathscr{R}_{\mathrm{od}}^{w, s}\right) \cap V(z), r\right] .
$$

The moduli spaces $M_{\kappa}^{w}\left(X^{o} ; \mathscr{R}_{\mathrm{ev} / \mathrm{od}}^{w, s}\right)$ are oriented using the homologyorientation of $X$, as described in the Appendix. When $b^{+}(X)>2$ 
(so that $b^{+}\left(X^{o}\right)>1$ ), the usual argument shows that this class is independent of the choice of metric on $X^{\circ}$ and the data used to define the $V(z)$, as well as showing that the definition gives a linear map on $\mathbb{A}_{\delta}\left(X^{\circ}\right)$. The relevant points here are Lemma 4.4 and the last clause of Proposition 4.14. In the case $b^{+}(X)=2$, one must choose the Riemannian metric to avoid reducibles, and in this case the class we have defined may, apparently, depend on the metric. (See Corollary 5.8, however.)

Now consider the case of $W^{o}$. The argument is analogous to the previous case, using Corollary 4.21 rather than 4.16. Choose a manifold with boundary, $\mathscr{R}^{\sim}$, which is a deformation-retract of the irreducible representation variety, as above. Again we can assume that $\gamma \in \mathbb{A}_{\delta}\left(W^{o} ; \mathbb{Z}\right)$ is a monomial, with $\delta \leq \Delta$. If $\kappa<\frac{1}{4} n$ then Corollary 4.21 gives a generic metric and generic good transverse representatives $V(e \gamma)$ for $e \gamma$ so that

$$
M_{\kappa+1 / 4}^{\bar{w}}\left(W^{o} \# \overline{\mathbb{C P}}^{2} ; \mathscr{R}_{\mathrm{ev}}^{w, s} \amalg \mathscr{R}_{\mathrm{od}}^{w, s}\right) \cap V(e \gamma)
$$

is a smooth manifold of dimension $6 g-6+8 \kappa-\delta$. We consider only $\kappa$ satisfying $\kappa \leq \delta / 8$ (which certainly implies $\kappa<n / 4$ ), so that the dimension is not more than $6 g-6$. Corollary 4.21 implies that the frontier of

$$
r: M_{\kappa+1 / 4}^{\bar{w}}\left(W^{o} \# \overline{\mathbb{C P}}^{2} ; \mathscr{R}_{\mathrm{ev}}^{w, s} \amalg \mathscr{R}_{\mathrm{od}}^{w, s}\right) \cap V(e \gamma) \rightarrow \mathscr{R}_{\mathrm{ev}}^{w, s} \amalg \mathscr{R}_{\mathrm{od}}^{w, s}
$$

is contained in dimension $6 g-6+8 \kappa-\delta-2$, and the same is true also of the restriction

$$
r: r^{-1}\left(\mathscr{R}^{\sim}\right) \rightarrow \mathscr{R}^{\sim} .
$$

Applying Proposition 4.7 to this last map, we obtain a cohomology class

$$
\left[M_{\kappa+1 / 4}^{\bar{w}}\left(W^{o} \# \overline{\mathbb{C P}}^{2} ; \mathscr{R}^{\sim}\right) \cap V(e \gamma), r\right] \in H^{\delta-8 \kappa}\left(\mathscr{R}_{\mathrm{ev}}^{w, s}\right) \oplus H^{\delta-8 \kappa}\left(\mathscr{R}_{\mathrm{od}}^{w, s}\right) .
$$

Here we need to orient the normal to $r$, using the convention described in the Appendix. Note that the image of $r$ in this case may meet the boundary of $\mathscr{R}^{\sim}$.) We then set

$$
D_{W^{\circ}}^{w}(\gamma)=\sum_{\kappa \leq \delta / 8}\left[M_{\kappa+1 / 4}^{\bar{w}}\left(W^{o} \# \overline{\mathbb{C P}}^{2} ; \mathscr{R}^{\sim}\right) \cap V(e \gamma), r\right] .
$$


Again, the result is independent of the metric on $W^{o}$, and the other choices.

Last in this sequence, we consider the singular moduli spaces. Fix $p \geq 0$ satisfying the parity condition and $\gamma \in \mathbb{A}_{\delta}\left(W^{o} \backslash \Sigma\right)$ with $\delta \leq$ $\Delta-2 p$. Choose $\alpha$ small enough that the hypotheses of Corollary 4.24 are satisfied with $p_{0}=0$. Now choose a generic metric and good representatives $V(\gamma)$ so that we can use Corollary 4.24 as above to construct cocycles

$$
\left[M_{\kappa}^{w, p, \alpha}\left(W^{o}, \Sigma ; \mathscr{R}^{\sim}\right) \cap V(\gamma), r\right]
$$

in $H^{\delta-8 \kappa+2 p}\left(\mathscr{R}_{\mathrm{ev}}^{w}\right) \oplus H^{\delta-8 \kappa+2 p}\left(\mathscr{R}_{\mathrm{od}}^{w}\right)$ whenever $\kappa \leq(\delta+2 p) / 8$. (Recall that the dimension of the cut-down moduli space here is $6 g-6+8 \kappa-$ $\delta-2 p$.) We then define

$$
D_{W^{o}, \Sigma}^{w, p}(\gamma)=\sum_{\kappa \leq(\delta+2 p) / 8}\left[M_{\kappa}^{w, p, \alpha}\left(W^{o}, \Sigma ; \mathscr{R}^{\sim}\right) \cap V(\gamma), r\right] .
$$

Using the second clause of Proposition 4.23, it is again straightforward to check that this cocycle is independent of the choice of generic metric, $\alpha$ and good representatives.

The last clause of the Theorem, concerning the filtration, is an immediate consequence of the definition and the fact that the action $\kappa$ must be non-negative: we see, for example, that if $w=0$ and $\gamma \in \mathbb{A}_{\delta}\left(W^{o}\right)$, then

$$
\begin{gathered}
D_{W^{\circ}}^{w}(\gamma) \in H^{\delta}\left(\mathscr{R}_{\mathrm{ev}}^{w, s}\right) \oplus H^{\delta-8}\left(\mathscr{R}_{\mathrm{ev}}^{w, s}\right) \oplus \cdots \\
\oplus H^{\delta}\left(\mathscr{R}_{\mathrm{od}}^{w, s}\right) \oplus H^{\delta-8}\left(\mathscr{R}_{\mathrm{od}}^{w, s}\right) \oplus \cdots \\
D_{W^{o, \Sigma}}^{w, p}(\gamma) \in H^{\delta+2 p}\left(\mathscr{R}_{\mathrm{ev}}^{w, s}\right) \oplus H^{\delta+2 p-8}\left(\mathscr{R}_{\mathrm{ev}}^{w, s}\right) \oplus \cdots \\
\oplus H^{\delta+2 p}\left(\mathscr{R}_{\mathrm{od}}^{w, s}\right) \oplus H^{\delta+2 p-8}\left(\mathscr{R}_{\mathrm{od}}^{w, s}\right) \oplus \cdots
\end{gathered}
$$

To prove the assertion regarding the equality between the pairings and the invariants of the closed manifolds, we need to check three things. First, we need to check that the algebraic pairing of these cycles agrees with the signed count of points in the fibered product of the moduli spaces. This is the content of Lemma 4.9. Second, we need to check that all points in the fibered product are accounted for in the pairing. This is a straightforward dimension count, along the lines of Propositions 4.14 and 4.19. We will go through the argument in the case of the singular invariants and where there are no homology 
classes coming from $X^{o}$ (i.e. $z=1$ ), and leave the reader to check the remaining cases.

We begin by choosing appropriate generic metrics on the cylindricalend manifolds $X^{o}$ and $W^{o}$. Choose $\gamma \in \mathbb{A}_{\delta}\left(W^{o}\right)$ where $\delta \leq \Delta-2 p$, and $\alpha$ satisfying inequalities (iv) and (v) of Proposition 4.23. Next choose a generic metric on $W^{o}$ so that the conclusions of Proposition 4.23 hold for all the cut-down moduli spaces

$$
M_{\kappa}^{w, p^{\prime}, \alpha}\left(W^{o}, \Sigma ; \mathscr{R}_{\mathrm{ev}}^{w, s} \amalg \mathscr{R}_{\mathrm{od}}^{w, s}\right) \cap V\left(\gamma^{\prime}\right),
$$

where $p^{\prime} \geq-\frac{1}{2} n$ and $\kappa \leq(\delta+2 p) / 8$, and $\gamma^{\prime}$ is a factor of $\gamma$ in $\mathbb{A}\left(W^{o} \backslash \Sigma\right)$. Write $g_{-}$for this metric on $W^{o}$. By Theorem 9.0.5 of [36], we can choose a metric $g_{+}$on $X^{\circ}$ so that the boundary maps

$$
r: M_{\kappa^{\prime}}^{w}\left(X^{o} ; \mathscr{R}_{\mathrm{ev} / \mathrm{od}}^{w, s}(Y)\right) \rightarrow \mathscr{R}_{\mathrm{ev} / \mathrm{od}}^{w, s}(Y)
$$

are transverse to all the sets of the form

$$
\stackrel{\leftarrow}{T}_{\kappa^{N}} \circ \cdots \circ \overleftarrow{T}_{\kappa^{1}}\left(r\left(M_{\kappa^{0}}^{w, p^{\prime}, \alpha}\left(W^{o}, \Sigma\right) \cap V\left(\gamma^{\prime}\right)\right)\right) \cap \mathscr{R}_{\mathrm{ev} / \mathrm{od}}^{w, s}
$$

for all $p^{\prime} \geq-\frac{1}{2} n$.

Now consider a sequence of metrics $g_{i}$ on $X$ so that $\left(X, g_{i}\right)$ contains a cylinder isometric to $[-i, i] \times Y$ and so that this sequence of metric converges uniformly on compact sets to $g_{+}$on $X^{\circ}$ and $g_{-}$on $W^{\circ}$. Consider the Uhlenbeck limit, as in Theorem 4.10, of a sequence of connections in

$$
M_{\kappa}^{w, p, \alpha}(X, \Sigma)_{g_{i}} \cap V\left(\jmath_{*}(\gamma)\right)
$$

where $\delta(\kappa)=\delta+2 p$, so that the intersection is zero-dimensional and this cut-down moduli space defines $D_{X, \Sigma}^{w, p}\left(\jmath_{*}(\gamma)\right)$. For such a limit, an obvious variant of Theorem 4.10 implies that there is a $\kappa$-appropriate sequence $\kappa^{-}, \kappa^{1}, \ldots, \kappa^{N}$ and a $p^{\prime}$ so that the intersection

$$
r\left(M_{\kappa_{+}}^{w}\left(X^{o}\right)\right) \cap \overleftarrow{T}_{\kappa^{N}} \circ \cdots \circ \overleftarrow{T}_{\kappa^{1}}\left(r\left(M_{\kappa^{-}}^{w, p^{\prime}, \alpha}\left(W^{o}, \Sigma\right) \cap V\left(\gamma^{\prime}\right)\right)\right)
$$

is non-empty. Suppose also that there are $j$ points of $W^{\circ} \backslash \Sigma$ (counted with multiplicity) where the limiting sequence bubbles, and $s$ points of $\Sigma$ where bubbling occurs. (Note that since $\gamma \in \mathbb{A}_{\delta}\left(W^{o} \backslash \Sigma\right)$, the 
corresponding constraint does not disappear when bubbling occurs at points of $\Sigma$.) We also have

$$
\kappa^{+}+\kappa^{0}+\kappa^{1}+\cdots+\kappa^{N}-\frac{1}{4} p^{\prime} \leq \kappa-\frac{1}{4} p
$$

using (2.25), and $\operatorname{deg}\left(\gamma^{\prime}\right) \geq \delta-5 j$ by (2.7). If $N>0$, then using Proposition 4.23 we see that the formal dimension of this intersection is

$$
\begin{aligned}
\delta\left(\kappa^{+}\right)+6 g-6+8\left(\kappa^{-}+\kappa^{1}+\right. & \left.\cdots+\kappa^{N}\right)-2 p^{\prime}-\operatorname{deg}\left(\gamma^{\prime}\right)-2-6 g-6 \\
& \leq \delta(\kappa)-2 p-\delta-3 j-2 s-2 \\
& \leq-2
\end{aligned}
$$

and hence, by the transversality assumptions we have made, this intersection is empty. If $N=0$, then the formal dimension of this intersection is

$$
\begin{aligned}
\delta\left(\kappa^{+}\right)+8 \kappa^{-}-2 p^{\prime}-\operatorname{deg}\left(\gamma^{\prime}\right)-6 g-6 & \leq \delta(\kappa)-2 p-\delta-3 j-2 s \\
& =-3 j-2 s .
\end{aligned}
$$

Thus our transversality assumptions imply that $j=s=0$ and $\kappa=$ $\kappa^{+}+\kappa^{-}$. In other words, any connection arising in such an Uhlenbeck limit is a point of the fibered product

$$
M_{\kappa_{+}}^{w}\left(X^{o} ; \mathscr{R}_{\mathrm{ev} / \mathrm{od}}^{w, s}\right) \times_{\left(r_{+}, r_{-}\right)}\left(M_{\kappa_{-}}^{w, p, \alpha}\left(W^{o}, \Sigma ; \mathscr{R}_{\mathrm{ev} / \mathrm{od}}^{w, s}\right) \cap V(\gamma)\right),
$$

where $\kappa_{+}+\kappa_{-}=\kappa$, and where $\delta\left(\kappa^{+}\right) \geq 0$ and $\kappa_{-} \geq 0$. Now $\delta(\kappa)=\delta+2 p$ so we have

$$
0 \leq \kappa^{-} \leq \frac{1}{8}(2 p+\delta) \leq \frac{1}{8} \Delta .
$$

Hence all points in the fibered product appear in the range where the pairing is defined.

Finally, we need to see that the points the union of the zero-dimensional fibered products (4.31), as $\kappa^{-}$runs through the finite range indicated, is diffeomorphic, in an orientation-preserving way, to the moduli space (4.30) which defines the invariant on the left-hand-side of the pairing formula, once the neck-length $i$ is sufficiently large. Given the above analysis of dimensions, this now follows from the gluing theorem, Theorem 1 of [35]. q.e.d. 
We also have a vanishing theorem for the case of negative $p$. (See also [27] and [25].)

Proposition 4.33. Let $(X, \Sigma)$ be as above, and assume $n$ and $g$ are such that $\Delta=\Delta(n, g)$ is non-negative (4.26). Then for all $p<0$ and all $z \in \mathbb{A}(X / \Sigma)$, the invariant $D_{X, \Sigma}^{w, p}(z)$ is zero.

Proof. Fix $p<0$ and choose $\alpha$ so small that the inequalities of Corollary 4.24 hold with $p_{0}=p$. Apply the argument used to establish the pairing formula in the proof of Theorem 4.28 above, taking a sequence of Riemannian metrics $g_{i}$ with increasing neck-length, but now with $\gamma=1$ (so that $\delta=0$ ). The conclusion (4.32) is the same; but now, because $p$ is negative and $\delta$ is zero, there is no $\kappa^{-}$which can arise, and one concludes that the moduli space is empty once $i$ is sufficiently large.

Remarks. (a) In the course of the proof of (4.28), we have seen that the invariants of the manifolds $X^{\circ}$ and $W^{o}$ are independent of the choice of perturbation of the metric, subject to the transversality requirements. These perturbations, however, are still compactly supported. We can also consider changing the metric on $Y$ (and hence on the cylindrical ends), subject to the requirements of the correspondence theorems in section 3. This class of metrics is connected, so one easily shows that the invariants are also independent of this choice: the relevant observations are that a version of the compactness theorem (4.10) continues to hold when the metric on the end is varied, and that Corollary 3.3 and Propositions 3.4-3.6 have variants involving a one-parameter family of metrics, as described at the end of section 3 . As a consequence, the invariants of $W^{\circ}$, for example, depend only on the topology of the tubular neighbourhood $N(\Sigma)$ and the chosen linebundle $w \rightarrow \Sigma$. The automorphisms of $w$ covering the identity map on the base act trivially on $F^{w}(Y)$. So $\operatorname{Diff}^{+}(\Sigma)$ acts on the Floer homology, and we have, for example:

Proposition 4.34. The map $D_{W^{\circ}}^{w}: \mathbb{A}(\Sigma)_{(\Delta)} \rightarrow \bar{F}^{w}(Y)$ respects the action of $\operatorname{Diff}^{+}(\Sigma)$. For $\gamma=1$ and all $p \leq \frac{1}{2} \Delta$, the element

$$
D_{W^{o}, \Sigma}^{w, p}(1) \in \bar{F}^{w}(Y)
$$

is invariant under the action of $\operatorname{Diff}^{+}(\Sigma)$.

(b) There are some homology classes on $X$ which lie in the image of both $\imath_{*}$ and $\jmath_{*}$. These are the classes coming from the first homology of $\Sigma$ (if their image is non-zero in $X$ ) and the class $x$ of a point in $X$. 
The pairing formulae allow us to express the values of the invariants of $X$ or $(X, \Sigma)$ on these classes in more than one way. In particular, since the image of the homology of $X^{o}$ contains the image of the homology of $W^{o} \backslash \Sigma$, all values of the invariant $D_{X, \Sigma}^{w, p}$ can be calculated from the second pairing formula of the Theorem with $\gamma=1$.

(c) In the pairing formulae, one of the manifolds involved has always been the standard manifold $W^{\circ}$. There is no real need for this, except that, if the manifold is changed, then some of the dimension and action formulae change too. Consider a 4 -manifold $Z^{\circ}$ with a cylindrical end modelled on $\bar{Y}(n, g)$, with $n>0$. Write

$$
q\left(Z^{\circ}\right)=(2 g-2)-\left(b^{1}\left(Z^{o}\right)-b^{+}\left(Z^{o}\right)-1\right),
$$

so that $q\left(W^{\circ}\right)=0$. The formal dimension of the moduli space $M_{\kappa}^{w}\left(Z^{o} ; \mathscr{R}_{\mathrm{ev} / \mathrm{od}}^{w, s}\right)$ is then

$$
\operatorname{dim} M_{\kappa}^{w}\left(Z^{o} ; \mathscr{R}_{\mathrm{ev} / \mathrm{od}}^{w, s}\right)=8 \kappa+6 g-6-3 q .
$$

The moduli spaces of ASD connections on $Z^{\circ}$ can be used to define invariants which take the form of linear functions

$$
D_{Z^{\circ}}^{w}: \mathbb{A}\left(Z^{o}\right)_{(\Delta-3 q)} \rightarrow \bar{F}^{w}(Y),
$$

where $\Delta(n, g)$ is as before. There are only a couple of points to mention. First, reducible connections are irrelevant as we are always considering connections on $Z^{o}$ with irreducible boundary values. The dimensioncounting arguments, used in the definition of the invariants of $W^{o}$, continue to show that ideal connections which involve intermediate reducible flat connections on $Y$ do not contribute more than codimension 2 to the frontier of the image of the moduli spaces in $\mathscr{R}_{\mathrm{ev} / \mathrm{od}}^{w, s}$. For the twisted reducibles, we used an argument that was special to $W^{\circ}$, because it was convenient to do so. However, one can deal with them in the same way that one treats the reducibles: there are only $2 g-2$ dimensional tori of twisted reducibles in $\mathscr{R}_{\mathrm{ev} / \mathrm{od}}^{w, s}$, and they cannot contribute anything of codimension smaller than $\Delta+2$ to the frontier. Similar remarks apply to a pair $\left(Z^{o}, \Sigma\right)$.

In section 9, we will make use of a rather trivial case, $Z^{\circ}=W^{o} \# \overline{\mathbb{C P}}^{2}$, in proving the blow-up formula for manifolds of simple type.

(d) Having completed the construction of the relative invariants, we can now look again, with the benefit of hind-sight, at the inequalities 
in section 3(i), such as in Corollary 3.3. Had $Y$ not been a circle bundle, one would have expected the image under $r$ of the moduli spaces $M_{\kappa}\left(X^{o} ; \mathscr{R}_{\mathrm{ev} / \mathrm{od}}^{w, s}\right)$ to have a frontier of codimension 1 , contributed by ideal instantons of length 1 , consisting of an instanton $\left[A^{\circ}\right]$ of charge $\kappa-m$ on $X^{o}$ and an instanton of charge $m$ on the cylinder. Each moduli space would not define a cycle, but the union of the moduli spaces of different charge would need to be considered together, as defining a class in a full Floer homology theory whose boundary map involved instantons on the cylinder. Such a Floer theory for circle bundles over Riemann surfaces has been constructed by Taubes [42].

\section{Expressing the invariants of the pair in terms of the Donaldson invariants}

(i) The invariants of $W^{\circ}$ are an isomorphism in low degree. Fix a line bundle $w \rightarrow \Sigma$, and let $\mathscr{B}^{*, w}(\Sigma)$ denote the space of (determinant one) gauge equivalence classes of irreducible connections in $\mathfrak{g}_{E}$, where $E \rightarrow \Sigma$ is a unitary rank-2 bundle with $\operatorname{det}(E)=w$. Let

$$
\mu_{\Sigma}: \mathbb{A}(\Sigma) \rightarrow H^{*}\left(\mathscr{B}^{w, *}(\Sigma)\right)
$$

denote the algebra homomorphism which extends the map

$$
\beta \mapsto-\frac{1}{4} p_{1}(\mathbf{U}) /[\beta],
$$

where $\mathbf{U}$ is the universal $\mathrm{SO}(3)$ bundle. According to [2], the inclusion of the set of flat connections, $\iota^{*}: \mathscr{R}^{w, s}(\Sigma) \rightarrow \mathscr{B}^{*, w}(\Sigma)$, induces an isomorphism in homology or cohomology in degrees less than $2 g-1$ if $w \cdot[\Sigma]$ is odd and in degrees less than $(2 g-3)$ if $w \cdot[\Sigma]$ is even. In particular we have the following:

Proposition 5.2. The map $\mu_{\Sigma}$ gives rise to isomorphisms,

$$
H^{\delta}\left(\mathscr{R}^{w, s}(\Sigma) ; \mathbb{R}\right) \equiv \mathbb{A}_{\delta}(\Sigma)
$$

and

$$
H_{\delta}\left(\mathscr{R}^{w, s}(\Sigma) ; \mathbb{R}\right) \equiv\left(\mathbb{A}_{\delta}(\Sigma)\right)^{*}
$$

for $\delta \leq 2 g-4$.

Let $p: Y \rightarrow \Sigma$ be, as usual, the circle bundle over $\Sigma$ of degree $n$. By pull-back, $p$ gives an isomorphism $p^{*}$ from $\mathscr{R}^{w, s}(\Sigma)$ to $\mathscr{R}_{\mathrm{ev}}^{w, s}(Y)$. The 
cohomology of the latter in dimensions $\delta \leq \Delta$ defines the even part of the truncated Floer cohomology $\bar{F}_{\mathrm{ev}}^{w}(Y)$ (see section 4(iii)), so we have:

Corollary 5.3. The maps $\mu_{\Sigma}$ and $p^{*}$ give rise to an isomorphism

$$
\nu: \mathbb{A}(\Sigma)_{(\Delta)} \rightarrow \bar{F}_{\mathrm{ev}}^{w}(Y) .
$$

Let $W^{o}$ be the usual disk bundle over $\Sigma$, with a cylindrical-end metric. Note that we identify $\mathbb{A}\left(W^{o}\right)$ with $\mathbb{A}(\Sigma)$ from this point on.

Proposition 5.4. The image of the relative Donaldson invariant

$$
D_{W^{\circ}}^{w}: \mathbb{A}(\Sigma)_{(\Delta)} \rightarrow \bar{F}^{w}(Y)
$$

is contained in the even part, $\bar{F}_{\mathrm{ev}}^{w}(Y) \subset \bar{F}^{w}(Y)$. The same is true for the invariants of the pair, $D_{W^{\circ}, \Sigma}^{w, p}$.

Proof. From the definition of the invariants, to prove the first statement it is enough to show that for suitable metric on $W^{o} \# \overline{\mathbb{C P}}^{2}$, the moduli spaces

$$
M_{\bar{\kappa}}^{\bar{w}}\left(W^{o} \# \overline{\mathbb{C P}}^{2} ; \mathscr{R}_{\text {od }}^{w, s}\right)
$$

are empty for $\kappa<n / 4$. For this, it is enough to show that the moduli spaces

$$
M_{\kappa}^{w}\left(W^{o} ; \mathscr{R}_{\mathrm{od}}^{w, s}\right)
$$

are empty for some metric. For a conformally-Kähler, cylindrical-end metric, a connection $[A]$ in this moduli space gives rise to a holomorphic bundle $\mathcal{E} \rightarrow W$, by Guo's extension theorem (3.12). Here $W$ is the (closed) ruled surface. The determinant of $\mathcal{E}$ is given by $\operatorname{det} \mathcal{E}=w+$ $\left[\Sigma_{-}\right]$as a $C^{\infty}$ bundle, so it has odd degree on the two-sphere fibres. At the end of this section (Proposition 5.11), we will show that $\mathcal{E}$ satisfies the following stability condition: if $\mathcal{F} \rightarrow \mathcal{E}$ is a non-zero map from a holomorphic line-bundle on $W$, then

$$
c_{1}(\mathcal{F}) \cdot\left[\Sigma_{+}\right] \leq \frac{1}{2} w \cdot\left[\Sigma_{+}\right] .
$$

Because the degree is odd on the fibres, the generic splitting type of $\mathcal{E}$ is at least $1 / 2$. From this and the stability condition, one can easily deduce that $c(\mathcal{E}) \geq n / 4$ using the argument of Lemma 3.23. Since $c(\mathcal{E})=\kappa$, it follows that the moduli spaces are empty for $\kappa<n / 4$ as claimed. 
Similarly, the second statement of the Proposition will follow if we can show that the moduli spaces

$$
M_{\kappa}^{w, p, \alpha}\left(W^{o}, \Sigma ; \mathscr{R}_{\mathrm{od}}^{w, s}\right)
$$

are empty when $\kappa<\frac{1}{8} \Delta<\frac{1}{4} n$, for some sufficiently small $\alpha$ and some cylindrical-end metric (now with a small cone-angle $2 \pi / \nu$ along $\Sigma$ ). If the metric is conformally Kähler and $\alpha$ is rational with denominator $\nu$, we can use Guo's extension theorem and Proposition 8.18 of [27] to show that a connection $[A]$ in this moduli space gives rise to a holomorphic bundle $\mathcal{E} \rightarrow W$ with $c(\mathcal{E})=\kappa$ and $\operatorname{det}(\mathcal{E})=w \otimes\left[\Sigma_{-}\right]$. Furthermore $\mathcal{E}$ has a holomorphic line subbundle $\left.\mathcal{L} \subset \mathcal{E}\right|_{\Sigma_{+}}$and

$$
\begin{aligned}
\operatorname{deg}_{\omega}(\mathcal{L})-\frac{1}{2} w \cdot\left[\Sigma_{+}\right] & =\frac{1}{2}(p-(g-1)) \\
& =-l,
\end{aligned}
$$

where $l$ is the monopole number. As in the ordinary case above, the pair $(\mathcal{E}, \mathcal{L})$ satisfies a stability condition $(5.14)$, which implies the condition (5.5) above when $\alpha$ is small. It follows again that the moduli spaces are empty for $\kappa<n / 4$.

By the first part of this Proposition, the invariant $D_{W^{\circ}}^{w}$ induces a map

$$
D: \mathbb{A}(\Sigma)_{(\Delta)} \rightarrow \bar{F}_{\mathrm{ev}}^{w}(Y) .
$$

Let $D=\sum_{i=0} D^{(8 i)}$ be the decomposition of $D$ which arises from the grading of $\bar{F}_{\mathrm{ev}}^{w}(Y)$. That is, using the definition of the latter as

$$
\bigoplus_{\delta \leq \Delta} H^{\delta}\left(\mathscr{R}_{\mathrm{ev}}^{w, s}\right)
$$

we write $D^{(8 i)}$ for the map

$$
D^{(8 i)}: \mathbb{A}_{\delta}(\Sigma) \rightarrow H^{\delta-8 i}\left(\mathscr{R}_{\mathrm{ev}}^{w, s}\right) \quad(i \leq \delta / 8),
$$

(see (4.29)).

Proposition 5.6. The top-degree part of the relative invariant of $W^{o}$ is given by

$$
D^{(0)}=\nu .
$$


Proof. By definition,

$$
D^{(0)}(\gamma)=\left[M_{1 / 4}^{\bar{w}}\left(W^{o} \# \overline{\mathbb{C P}}^{2} ; \mathscr{R}^{\sim}\right) \cap V(e \gamma), r\right],
$$

where $\mathscr{R}^{\sim} \subset \mathscr{R}_{\mathrm{ev}}^{w, s}$ is a compact submanifold with boundary which is a deformation retract. Here the connected sum $W^{\circ} \# \overline{\mathbb{C P}}^{2}$ is formed with a long neck. We claim that if $C \subset \mathscr{R}_{\mathrm{ev}}^{w, s}$ is any compact set, then for all neck lengths $L$ sufficiently large, the moduli space

$$
M_{C}=M_{1 / 4}^{\bar{w}}\left(W^{o} \# \overline{C P}^{2} ; C\right)
$$

is compact. If not then there is a sequence of tube lengths $L_{i}$ going to infinity and ideal connections $\left(\left[A_{0}^{i}\right],\left[A_{1}^{i}\right], \ldots,\left[A_{n^{i}}^{i}\right]\right)$ with compatible boundary values and $r_{+}\left(\left[A_{n^{i}}^{i}\right]\right) \in C$, with total action $\frac{1}{4}$ and each $n_{i} \geq 1$. (Since the original total action is smaller than 1 there can be no bubbling at points and hence the total action of the ideal connection is preserved under weak limits). Taking the Uhlenbeck limit of this sequence of ideal connections, we get an ideal connection $\left(\left[A_{0}\right],\left[A_{1}\right], \ldots,\left[A_{n}\right]\right)$ with $n \geq 1$ on $W^{o}$ and a connection $[B]$ on $\overline{C P}^{2}$. Then action of $[B]$ is necessarily $\frac{1}{4}$ so the ideal connection $\left(\left[A_{0}\right],\left[A_{1}\right], \ldots,\left[A_{n}\right]\right)$ with compatible boundary values is flat, and hence we must have had $n=0$, a contradiction.

Once one knows that the moduli space is compact, standard gluing arguments show that $M_{C}$ has the structure of a two sphere bundle over $M_{0}^{w}\left(W^{o}, C\right)$. The end-limit map $r: M_{0}^{w}\left(W^{o}, C\right) \rightarrow C$ identifies the base of the bundle with $C$. To prove the result it suffices to show that for any $\gamma$ satisfying the degree constraint, and taking $C$ to represent a cycle in $\mathscr{R}^{\sim}$, we have

$$
\sharp\left(M_{C} \cap V(e \gamma)\right)=\left\langle\mu_{\Sigma}(\gamma), C\right\rangle .
$$

Since $M_{C}$ is compact, the algebraic intersection of $M_{C}$ with the good representative $V(e \gamma)$ is simply the ordinary evaluation of the cohomology class $\mu(e \gamma)$ on $\left[M_{C}\right]$ :

$$
\sharp\left(M_{C} \cap V(e \gamma)\right)=\left\langle\mu(e \gamma),\left[M_{C}\right]\right\rangle .
$$

By the blow-up formula, the class $\mu(e)$ evaluates to 1 on the fibers of $r$ and the result is immediate. q.e.d.

This implies the following result. 
Corollary 5.7. The even part of the relative Donaldson invariant,

$$
D: \mathbb{A}(\Sigma)_{(\Delta)} \rightarrow \bar{F}_{\mathrm{ev}}^{w}(Y)
$$

is an isomorphism.

Proof. By the preceding results, $D$ respects the filtration and its top-degree part is an isomorphism. q.e.d.

There is another loose end which we can now tidy up. In the definition of the invariant $D_{X^{\circ}}^{w}$ in Theorem 4.28 , the possibility was left open that the invariant depended on the choice of metric in the case $b^{+}(X)=2$.

Corollary 5.8. The invariant $D_{X}^{w}$ o is independent of the choice of Riemannian metric, even when $b^{+}(X)=2$.

Proof. Consider first the part of the invariant taking values in $F_{\mathrm{ev}}^{w}$ :

$$
D_{X^{\circ}, \mathrm{ev}}^{w}(z) \in F_{\mathrm{ev}}^{w},
$$

for some $z \in \mathbb{A}\left(X^{o}\right)$. From the first of the pairing formulae in Theorem 4.28 and the fact that odd part of $D_{W^{\circ}}^{w}$ vanishes, we have

$$
D_{X}^{w}\left(\imath_{*}(\gamma) \jmath_{*}(z)\right)=\left\langle D(\gamma), D_{X^{\circ}, \mathrm{ev}}^{w}(z)\right\rangle,
$$

for all $\gamma \in \mathbb{A}\left(W^{o}\right)_{(\Delta)}$, where $D$ is as in the previous Corollary. The lefthand side is independent of metric since $b^{+}(X)>1$. By the previous Corollary and the fact that the pairing between $\bar{F}^{w}$ and $F^{w}$ is perfect, we deduce that $D_{X^{\circ}, \mathrm{ev}}^{w}(z)$ is also metric-independent. For the odd component, one can identify $D_{X^{\circ}, \text { od }}^{w}$ with $\pm D_{X^{\circ}, \mathrm{ev}}^{w^{\prime}}$, where $w^{\prime}=w-[\Sigma]$. q.e.d.

(ii) The existence of a universal formula. Combining Proposition 5.4 and Corollary 5.7, we obtain:

Proposition 5.9. For all $n>0$, all $g>1$ and all $p \leq \frac{1}{2} \Delta(n, g)$, there is a unique inhomogeneous element $\omega_{p}^{w}=\omega_{p}^{w}(n, g) \in \mathbb{A}(\Sigma)_{(2 p)}$ so that as elements of Floer cohomology

$$
2^{g} D_{W^{o}}^{w}\left(\omega_{p}^{w}\right)=D_{W^{o}, \Sigma}^{w, p}(1) .
$$

(The factor $2^{g}$ is introduced for later convenience.) The material of sections 3,4 and 5 has lead us to the following theorem: 
Theorem 5.10. Let $(X, \Sigma)$ be an admissible pair, such that $X^{o}$ is an admissible cylindrical-end manifold. Suppose the genus $g(\Sigma)$ is at least 2 and the class $S$ represented by $\Sigma$ has $Q(S)=n>0$. Let $\imath: \Sigma \rightarrow X$ and $\jmath: X / \Sigma \rightarrow X$ be the inclusions. Suppose that the quantity

$$
\Delta(n, g)=\min \{2 g-4-(n / 2), 2 n-2\}
$$

is non-negative. Let $p$ be a non-negative integer satisfying the parity condition (2.20), so that $D_{X, \Sigma}^{w, p}$ is defined, and suppose that

$$
p \leq \frac{1}{2} \Delta(n, g) .
$$

Then there is an element $\omega_{p}^{w} \in \mathbb{A}(\Sigma)$, inhomogeneous of degree $2 p$, such that

$$
D_{X, \Sigma}^{w, p}(z)=2^{g} D_{X}^{w}\left(\imath_{*}\left(\omega_{p}^{w}\right) \jmath_{*}(z)\right)
$$

for all $z \in \mathbb{A}(X / \Sigma)$. The element $\omega_{p}^{w}$ depends only on $n=Q(S)$, $g=\operatorname{genus}(\Sigma)$ and $w \cdot S$ mod 2 , and is uniquely characterized by Proposition 5.9. It does not depend on $X$ or $z$.

For $p<0$, the invariant $D_{X, \Sigma}^{w, p}(z)$ is zero for all $z \in \mathbb{A}(X / \Sigma)$.

Proof. Combine Proposition 5.9 with the pairing formulae in Theorem 4.28 to deduce the first statement. The vanishing of the invariants for $p<0$ was stated earlier as Proposition 4.33. $\quad$ q.e.d.

(iii) A stability condition. We now return to a matter which was postponed during the proof of Proposition 5.4. First, we describe a conformally-Kähler, cylindrical-end metric $g_{\text {cyl }}$ on $W^{o}$. The conformal factor will be of the form

$$
\sigma^{2}=\exp \left(-\frac{2 \pi n}{V} \tau\right)
$$

where $\tau: W^{o} \rightarrow \mathbb{R}$ is a function agreeing with the first coordinate on the end, $[0, \infty) \times \bar{Y}$, of $W^{o}$; so the corresponding Kähler metric $g$ is $\sigma^{2} g_{\text {cyl }}$. Such a metric can be written down explicitly by describing the Kähler form $\Phi$ for $g$ : we set

$$
\Phi=f^{\prime}(t) d t \wedge \eta+\left(\frac{2 \pi n}{V}\right) f(t) p^{*}\left(\Phi_{\Sigma}\right),
$$

where $t$ is the standard coordinate on the interval $(-\pi, \infty), \Phi_{\Sigma}$ is the Kähler form of a metric on $\Sigma$, and the other notation is as in (2.10). If 
we take $f$ to be a smooth, monotone decreasing function on $(-\pi, \infty)$ satisfying

$$
f(t)= \begin{cases}1-\cos t, & \text { when }-\pi<t<-\pi / 2 \\ e^{-2 \pi n t / V}, & \text { when } t>10,\end{cases}
$$

then it is not hard to verify that the form is closed, that the corresponding metric $g$ is positive and can be completed at $t=-\pi$ by attaching a copy, $\Sigma_{+}$, of $\Sigma$, and that $\sigma^{-2} g$ is cylindrical in the region $t>10$. Notice that because the conformal factor is exponentially decaying, $g$ has finite volume. Let $\Lambda: \Omega^{1,1}\left(W^{o}\right) \rightarrow \Omega^{0,0}\left(W^{o}\right)$ denote the contraction with $\Phi$.

Let $W$ be the closed ruled surface, obtained by attaching $\Sigma_{-}$at the end $\tau=\infty$. Notice that neither $g_{\text {cyl }}$ nor $g$ extends to a metric on $W$. Given an ASD connection $A$ in the moduli space $M_{\kappa}^{w}\left(W^{o} ; \mathscr{R}_{\mathrm{ev} / \mathrm{od}}^{w}\right)_{g}$, one obtains, as observed above, a holomorphic bundle $\mathcal{E}_{A} \rightarrow W$ whose restriction to $\Sigma_{-}$is semi-stable. If $\omega \rightarrow W$ is a holomorphic bundle whose underlying $C^{\infty}$ bundle is $w$, we can take $\operatorname{det} \mathcal{E}_{A}$ to be $\omega$ in the even case and $\omega \otimes\left[\Sigma_{-}\right]$in the odd case.

Proposition 5.11 If $s: \mathcal{F} \rightarrow \mathcal{E}_{A}$ is a non-zero map from a holomorphic line bundle on $W$, then

$$
c_{1}(\mathcal{F}) \cdot\left[\Sigma_{+}\right] \leq \frac{1}{2} \omega \cdot\left[\Sigma_{+}\right]
$$

Remark. One can interpret the conclusion as saying that $\mathcal{E}$ is semi-stable with respect to the degenerate Kähler class $\left[\Sigma_{+}\right]$, defined by $\Phi$.

Lemma 5.12. Let $\mathcal{F} \rightarrow W$ be a holomorphic line bundle with Hermitian metric $h$ and compatible connection $B$.

(i) There is a complex gauge transformation $\gamma$ of $\left.\mathcal{F}\right|_{W^{\circ}}$ so that $\Lambda F_{\gamma^{*}(B)}$ is a constant multiple of $\sigma^{-2}$ :

$$
i \Lambda F_{\gamma^{*}(B)}=c \sigma^{-2} \text {. }
$$

(ii) The norm of $\gamma$ measured by $\left.h\right|_{W^{\circ}}$ is bounded above by

$$
C e^{\frac{c-\beta}{\delta} \tau}
$$

where $\delta=\frac{2 \pi n}{V}$ and $\beta$ has the same sign as $c_{1}(\mathcal{F}) \cdot\left[\Sigma_{-}\right]$. 
(iii) The sign of $c$ is the same as the sign of $c_{1}(\mathcal{F}) \cdot\left[\Sigma_{+}\right]$.

Proof. First we compare the metrics $g$ and $g_{\text {cyl }}$. Let $*_{g}$ denote the Hodge star operator for the metric $g$ and let $*_{\text {cyl }}$ denote the Hodge star operator for the metric $g_{\text {cyl }}$. On $p$-forms these are related by

$$
\begin{aligned}
*_{g} & =\sigma^{4-2 p_{\mathrm{cyl}}} \\
& =\exp \left(-(4-2 p) \frac{\pi n}{V} \tau\right) *_{\mathrm{cyl}} .
\end{aligned}
$$

Thus the $L^{2}$-norm with respect to $g$ on functions is given by

$$
\|f\|_{g}^{2}=\int_{W^{\circ}} f^{2} \exp \left(-\frac{4 \pi n}{V} \tau\right) *_{\mathrm{cyl}} 1 .
$$

We write $\sigma L^{2}$ for the space $\left\{\sigma f \mid f \in L^{2}\right\}$ with the norm $\left\|\sigma^{-1} f\right\|_{L^{2}}$. So the norm of an element $f \in \sigma L^{2}\left(W^{0}, g\right)$ is

$$
\left\|\sigma^{-1} f\right\|_{g}^{2}=\int_{W^{\circ}} f^{2} \exp \left(-\frac{2 \pi n}{V} \tau\right) *_{\mathrm{cyl}} 1 .
$$

We can compare these norms to the weighted Sobolev spaces of [36] and [40]. These norms are defined by

$$
\|f\|_{L_{k, \delta^{\prime}}^{2}}=\int_{W^{\circ}} \sum_{i=0}^{k}\left|\nabla^{i} f\right|^{2} e^{\delta^{\prime} \tau}
$$

where $\nabla$ is the covariant derivative for the cylindrical metric. The completion of $C_{0}^{\infty}\left(W^{o}\right)$ with respect to these norms is denoted $L_{k, \delta^{\prime}}^{2}\left(W^{o}, g^{\mathrm{cyl}}\right)$. Thus $\sigma L^{2}\left(W^{o}, g\right)=L_{\delta^{\prime}}^{2}\left(W^{o}, g^{\mathrm{cyl}}\right)$ where $\delta^{\prime}=-2 \pi n / V$.

The Laplacian is given by

$$
\Delta_{g} f=-\sigma^{-4} *_{\text {cyl }} d *_{\text {cyl }} \sigma^{2} d,
$$

so $\sigma^{2} \Delta_{g}$ is the Laplace operator associated with the weighted Sobolev spaces $\sigma L^{2}\left(W^{o}, g\right)$. For part (i), we need the following Lemma:

Lemma 5.13. For $\delta^{\prime}$ in the interval $(-2 \delta, 0)$ the operator

$$
\sigma^{2} \Delta_{g}: L_{2, \delta^{\prime}}^{2}\left(W^{o}, g_{\mathrm{cyl}}\right) \rightarrow L_{\delta^{\prime}}^{2}\left(W^{o}, g_{\text {cyl }}\right)
$$

is a Fredholm operator of index zero. The kernel of $\Delta_{g}$ on these spaces consists of the constant functions. 
Let $\lambda>0$ be the square root of the smallest positive eigenvalue of the Laplacian on functions on $Y$. Then for $\delta^{\prime}$ in the interval $(0, \lambda)$ the operator

$$
\sigma^{2} \Delta_{g}: L_{2, \delta^{\prime}}^{2}\left(W^{o}, g_{\mathrm{cyl}}\right) \rightarrow L_{\delta^{\prime}}^{2}\left(W^{o}, g_{\mathrm{cyl}}\right)
$$

is a Fredholm operator of index -1 . The kernel of $\Delta_{g}$ on these spaces is trivial.

Proof. On the cylinder $[0, \infty) \times Y$ the Laplace operator has the form

$$
\sigma^{2} \Delta_{g} f=-\frac{d^{2} f}{d t^{2}}+\delta \frac{d f}{d t}+\Delta_{Y} f
$$

where $\Delta_{Y}$ is the Laplacian for $Y$. It straight forward to apply the Fredholm theory of Lockhart and McOwen [32] to deduce that for $-2 \delta<$ $\delta^{\prime}<0$ this operator is Fredholm on $L_{\delta^{\prime}}^{2}$. For $\delta^{\prime}=-\delta$ the operator is formally self-adjoint and one can check then that it has index 0 . One also finds that this operator is Fredholm for $0<\delta^{\prime}<-\delta+\sqrt{\delta^{2}+4 \lambda^{2}}$. The index drops by one when $\delta^{\prime}$ becomes positive from the elimination of constant functions from the kernel.

To continue with the proof of Lemma 5.12 we may assume without loss of generality that $\left.h\right|_{\Sigma_{-}}$is Hermitian Einstein. For part (i), we seek a solution $\xi$ to the equation $\Delta_{g} \xi=c \sigma^{-2}-i \Lambda F$ or

$$
\sigma^{2} \Delta_{g} \xi=c-i \sigma^{2} \Lambda F \text {. }
$$

So by Lemma 5.13 and the Fredholm alternative, we find $\gamma$ satisfying (i) if we can show that $\sigma^{2} \Lambda F \in \sigma L^{2}\left(W^{0}, g\right)$. Let $\sigma^{-2} \Phi=\Phi_{\text {cyl }}$; note that $\Phi_{\text {cyl }}$ is bounded. Then

$$
\begin{aligned}
\Lambda F & =*_{g}(\Phi \wedge F) \\
& =\sigma^{-4} *_{\mathrm{cyl}}\left(\sigma^{2} \Phi_{\mathrm{cyl}} \wedge F\right) \\
& =\sigma^{-2} *_{\mathrm{cyl}}\left(\Phi_{\mathrm{cyl}} \wedge F\right) .
\end{aligned}
$$

Since $F$ bounded in the cylindrical norm, we see that $\sigma^{2} \Lambda F$ is also bounded and hence is in $\sigma L^{2}\left(W^{0}, g\right)$, as required.

For part (ii) we use the fact that $\sigma^{2} \Lambda F=*_{\text {cyl }}\left(\Phi_{\text {cyl }} \wedge F\right)$. Since $h$ is Hermitian Einstein on $\Sigma_{-}$there is a constant $\beta$ with the same sign as $c_{1}(\mathcal{F}) \cdot\left[\Sigma_{-}\right]$so that $i \sigma^{2} \Lambda F=\beta+O\left(e^{-a t}\right)$ for some $a>0$. Thus the $\xi$ found above satisfies

$$
\sigma^{2} \Delta_{g} \xi=c-\beta+O\left(e^{-a t}\right)
$$


Consider the function $\eta=\frac{(c-\beta)}{\delta} \tau$ on $W^{o}$. We have $\sigma^{2} \Delta_{g} \eta=c-\beta$ on the end of $W^{o}$ so $\xi-\eta$ satisfies

$$
\sigma^{2} \Delta_{g}(\xi-\eta) \in L_{\delta^{\prime}}^{2}
$$

for some $\delta^{\prime} \geq 0$. Thus by the second clause of Lemma 5.13 we can find $u \in L_{2, \delta^{\prime}}^{2}$ and $v \in L_{\delta^{\prime}}^{2}$ so that

$$
\sigma^{2} \Delta_{g}(\xi-\eta)=\sigma^{2} \Delta_{g} v+u .
$$

Now by the first clause there is a constant $d$ so that $\xi-\eta=d+v$ whence $\xi=\eta+d+v$, so $\gamma=e^{\xi}$ satisfies the required bound.

For part (iii), we have, on the one hand,

$$
\int_{W^{\circ}} i \Lambda F\left(*_{g} 1\right)=c \int_{W^{\circ}} \sigma^{-2}\left(*_{g} 1\right)
$$

and $\sigma^{-2}$ is integrable. On the other hand, we have

$$
\int_{W^{\circ}} i \Lambda F\left(*_{g} 1\right)=\int_{W^{\circ}} i F \wedge \Phi .
$$

The form $\Phi$ is self-dual and has finite energy, so by looking at it in the cylindrical-end metric and applying Proposition 4.9 from [3], we see that $\Phi$ represents a class in the image of $H_{c}^{2}\left(W^{o}\right)$ in $H^{2}\left(W^{o}\right)$. Hence $\Phi$ represents a positive multiple of $\left[\Sigma_{+}\right]$. Also, $i F_{\gamma^{*}(B)}$ represents the same class on $W^{o}$ as $i F_{B}$, so the last integral represents the pairing between $2 \pi c_{1}(\mathcal{F})$ and $\left[\Sigma_{+}\right]$.

Proof of Proposition 5.11. Given Lemma 5.12, the proof follows the familiar pattern (see for example [11]). We view the map $\mathcal{F} \rightarrow \mathcal{E}$ as a holomorphic section, $s$, of $\mathcal{F}^{*} \otimes \mathcal{E}$. Using the fact that $A$ (the connection in $\left.\mathfrak{g}_{E}\right)$ is ASD, and applying part (i) of Lemma 5.12 to $\operatorname{det}\left(\mathcal{F}^{*} \otimes \mathcal{E}\right)$, we can find a connection $C$ in $\mathcal{F}^{*} \otimes \mathcal{E}$ with

$$
i \Lambda F_{C}=c \sigma^{-2} .
$$

Viewed in terms of the cylindrical end metric and a Hermitian fibre metric, $h$, pulled back from $W$, the restriction of $s$ to $W^{o}$ is bounded. We wish to estimate $s$ with respect to the Hermitian Einstein metric $h_{H E}$ coming from the ASD connection. The comparison of $h$ with $h_{H E}$ comes in three steps. First there is a complex gauge transformation $\gamma_{0}$ 
arising from Lemma 3.15. To estimate the growth $\gamma_{0}$ we need to estimate the decay of the connection to its limiting value. If the boundary value is irreducible then $\gamma_{0}$ is trivial and there is nothing to estimate. If the boundary value is reducible then it follows as in the proof of Lemma 13.3.2 in [36] that in a neighborhood of a reducible connection $\Gamma$ we have the following Lojaszewicz inequality for a connection $A$ close enough to the flat connections and in Coulomb gauge:

$$
C \operatorname{dist}(A, \mathscr{R}(Y))^{3} \leq\left\|\nabla_{A} C S\right\|_{L^{2}}
$$

for some positive constant $C$. Here the distance is measured with respect to the $L_{1}^{2}$ metric. Thus

$$
\begin{aligned}
d t\left|A(t)-A_{\infty}\right| & \leq-\left\|\nabla_{A} C S\right\|_{L^{2}} \\
& \leq-C \operatorname{dist}\left(A(t),\left.\mathscr{R}(Y)\right|^{3}\right. \\
& \leq-C\left|A(t)-A_{\infty}\right|^{3} .
\end{aligned}
$$

Integrating this inequality gives

$$
\left\|A(t)-A_{\infty}\right\|_{L_{1}^{2}} \leq C_{1} \sqrt{t+C_{2}}
$$

for some positive constants $C_{1}$ and $C_{2}$. A straightforward bootstrap argument shows that this decay rate is valid for any stronger norm. Since $\gamma_{0}$ is obtained as the solution to the ODE in Lemma 3.15 we have

$$
\left|\gamma_{0}\right|_{h} \leq C_{3} e^{C_{4} \sqrt{t}} .
$$

Next is the gauge transformation $\gamma_{1}$ coming from the extension result. Since in terms of the cylindrical end metric this gauge transformation goes between connections exponentially decaying to the same connection, the gauge transformation exponentially approaches the identity.

Finally there is the gauge transformation $\gamma$ obtained in Lemma 5.12, coming from the determinant line of $\mathcal{F}^{*} \otimes \mathcal{E}$. Since both $\gamma_{0}$ and $\gamma_{1}$ have determinant $1, \operatorname{det}(\mathcal{F})^{*} \otimes \mathcal{E}$ is naturally still a line bundle over $W$ and the Lemma is applicable. Suppose now that $c \leq 0$. Since $\left.\mathcal{E}\right|_{\Sigma^{-}}$is semi-stable, $\beta \geq 0$. Thus Lemma 5.12 implies $\gamma$ is bounded.

Since $\bar{\partial}_{C} s=0$, the Weitzenböck formula for sections of $\mathcal{F}^{*} \otimes \mathcal{E}$ says that

$$
\frac{1}{4} \Delta\left|\gamma \gamma_{1} \gamma_{0} s\right|_{h}^{2}+\frac{1}{2}\left|\nabla_{C} \gamma \gamma_{1} \gamma_{0} s\right|_{h}^{2}=\left\langle\left(i \Lambda F_{C}\right) \gamma \gamma_{1} \gamma_{0} s, \gamma \gamma_{1} \gamma_{0} s\right\rangle_{h}
$$


where all norms and covariant derivatives are taken with respect to the Kähler metric $g$ while the unitary structure in $E$ is the one coming from the ASD connection. We must check that $\int \Delta\left|\gamma \gamma_{1} \gamma_{0} s\right|^{2}=0$. By Stokes' theorem, we have for all $T$,

$$
\int_{\tau \leq T} \Delta\left|\gamma \gamma_{1} \gamma_{0} s\right|_{h}^{2} *_{g} 1=\int_{\tau=T} *_{g} d\left|\gamma \gamma_{1} \gamma_{0} s\right|_{h}^{2}=\int_{\tau=T} \sigma^{3} *_{\mathrm{cyl}} d\left|\gamma \gamma_{1} \gamma_{0} s\right|_{h}^{2} .
$$

Because $d\left|\gamma \gamma_{1} \gamma_{0} s\right|_{h}^{2}$ grows at worst like $e^{\sqrt{t}}$ in the cylindrical norms, the boundary term goes to zero. So by integrating the Weitzenböck formula, we arrive at a contradiction if $c$ is not positive. But by part (iii) of Lemma 5.12, the sign of $c$ is the same as the sign of $-2 c_{1}(\mathcal{F})$. $\left[\Sigma_{+}\right]+c_{1}(\mathcal{E}) \cdot\left[\Sigma_{+}\right] . \quad$ q.e.d.

There is a version of Proposition 5.11 for the case of connections on $W^{o}$ with singularity along $\Sigma$. If $[A]$ belongs to the moduli space $M_{\kappa}^{w, p, \alpha}\left(W^{o}, \Sigma ; \mathscr{R}_{\mathrm{ev} / \mathrm{od}}^{w}\right)$, where $\alpha$ is rational with denominator $\nu$, one obtains a holomorphic bundle $\mathcal{E}=\mathcal{E}_{A}$ on $W$ with $c(\mathcal{E})=\kappa$ and $\operatorname{det} \mathcal{E}=$ $\omega$ or $\omega \otimes\left[\Sigma_{-}\right]$, together with a distinguished line sub-bundle $\left.\mathcal{L} \subset \mathcal{E}\right|_{\Sigma_{+}}$.

Proposition 5.14. Let $(\mathcal{E}, \mathcal{L})$ be as above. If $s: \mathcal{F} \rightarrow \mathcal{E}_{A}$ is a non-zero map from a holomorphic line bundle on $W$, then

$$
c_{1}(\mathcal{F}) \cdot\left[\Sigma_{+}\right]+\alpha n \leq \frac{1}{2} \omega \cdot\left[\Sigma_{+}\right]
$$

in the case that the image of $\left.s\right|_{\Sigma_{+}}$is contained in $\mathcal{L}$, and

$$
c_{1}(\mathcal{F}) \cdot\left[\Sigma_{+}\right]-\alpha n \leq \frac{1}{2} \omega \cdot\left[\Sigma_{+}\right]
$$

otherwise.

Proof. These inequalities are the condition for parabolic semistability of these bundles on $W$, with respect to the degenerate Kähler class $\left[\Sigma_{+}\right]$, and as such they can be regarded as an orbifold version of the previous result, Proposition 5.11, along the lines of [27]. See Proposition 8.20 of [27]. q.e.d.

\section{Calculating the universal formulae}

(i) The formulae. This section is devoted to a partial calculation of the universal elements $\omega_{p}^{w}$ in $\mathbb{A}(\Sigma)$ which appear in the formulae (5.10), 
expressing the invariants $D^{w, p}$ of a pair $(X, \Sigma)$ in terms of the invariants $D^{w}$ of $X$. As usual, we denote by $S$ the fundamental class of $\Sigma$ with its chosen orientation and by $x$ the positive generator of $H_{0}(\Sigma ; \mathbb{Z})$. We take $a_{1}, \ldots, a_{2 g}$ to be a standard basis for $H_{1}(\Sigma ; \mathbb{Z})$, with the convention that the non-zero intersection numbers are

$$
a_{i} \cdot a_{i+g}=-a_{i+g} \cdot a_{i}=1 .
$$

As elements of $\mathbb{A}(\Sigma)$, the classes $S, a_{i}$ and $x$ have degree 2,3 and 4 respectively. We write $\Gamma$ for the following element of degree 6 :

$$
\Gamma=\sum a_{i} a_{i+g} \in \mathbb{A}(\Sigma) .
$$

This element is independent of the choice of symplectic basis. The elements of $\mathbb{A}(\Sigma)$ which are invariant under the maps induced by all orientation-preserving diffeomorphisms of $\Sigma$ are precisely the elements of the polynomial algebra generated by $S, x$ and $\Gamma$.

Recall from section 5 that $\omega_{p}^{w}$ is an element of $\mathbb{A}(\Sigma)$ having non-zero terms only in degrees $2 p^{\prime}$, for $p^{\prime}$ satisfying $p^{\prime} \leq p$ and $p^{\prime} \equiv p \bmod 4$. We write

$$
\omega_{p}^{w}=\omega_{p, 0}^{w}+\omega_{p, 1}^{w}+\cdots,
$$

where $\omega_{p, i}^{w}$ is homogeneous of degree $2 p-8 i$. Each term is invariant under the diffeomorphism group by Proposition 4.34 so can be written as a polynomial in $S, x$ and $\Gamma$. Note that for $p<4$ there is only one term here, and $\omega_{p}^{w}$ is then homogeneous. Our first proposition gives these low examples in full. We again write $g$ for the genus and $n$ for the self-intersection number of $\Sigma$. According to Theorem 5.10, the coefficients of $\omega_{p}^{w}$ depend only on $g, n$ and $w(S) \bmod 2$. In fact, neither $n$ nor $w$ enter the formulae when $p<4$.

Proposition 6.2. For all $w$ and all intersection numbers $n$ in the range allowed by Theorem 5.10, we have

$$
\begin{aligned}
& \omega_{0}^{w}=1 \\
& \omega_{1}^{w}=S \\
& \omega_{2}^{w}=\frac{1}{2}\left(S^{2}-(g-1) x\right) \\
& \omega_{3}^{w}=\frac{1}{6}\left(S^{3}-(3 g-4) S x-\Gamma\right) .
\end{aligned}
$$

Remark. It should be understood here that $g$ and $w$ satisfy the parity condition $p \equiv(g-1)-w(S) \bmod 2(2.20)$ in order that $\omega_{p}^{w}$ is defined. 
For the proof of Theorem 1.7, we need only the first three of these four formulae, together with the following result:

Proposition 6.3. The coefficient of $S^{p}$ in $\omega_{p}^{w}$ is $1 / p$ !

The proof of these formulae occupies the remainder of section 6 . We shall see, in fact, that the top-degree part $\omega_{p, 0}^{w}$ can be calculated quite mechanically. In section 8 , where we prove some results about simple type, we will have need of one more in this sequence.

Proposition 6.4. The top-degree part, $\omega_{4,0}^{w}$, of $\omega_{4}^{w}$ is given by

$$
\omega_{4,0}^{w}=\frac{1}{24}\left(S^{4}-2(3 g-5) S^{2} x+3(g-1)(g-3) x^{2}-4 S \Gamma\right) .
$$

Calculating the lower terms $\omega_{p, i}^{w}$ for $i \geq 1$ is more difficult. At the time of writing, the authors believe they have a closed formula, modulo $\Gamma$, for the generating function of $\sum \omega_{p}^{w} s^{p} / p$ !, and hope to return to this in a later paper. The techniques and calculations which are presented here draw on unpublished work of J.L. Evans [12].

(ii) An index calculation. The method of calculation we shall employ is a generalization of the argument used in section 9 of [27]. Let $\Sigma$ be a Riemann surface of genus $g \geq 2$, and let $V$ be a compact, oriented manifold parametrizing a family of holomorphic rank-2 bundles with fixed determinant on $\Sigma$. More precisely, we suppose there is a rank-2 bundle $\mathbf{E} \rightarrow \Sigma \times V$ equipped with a $\bar{\partial}$ operator $\bar{\partial}_{v}$ on each slice $\Sigma \times\{v\}$, and we suppose that $\Lambda^{2} \mathbf{E}$ is isomorphic to $\omega \otimes K$, where $\omega$ is a fixed line bundle on $\Sigma$ and $K$ is pulled back from $V$. Let $\mathscr{E}_{v}$ denote the holomorphic bundle obtained from $\left.\mathbf{E}\right|_{\Sigma \times\{v\}}$ by equipping it with the $\bar{\partial}$ operator $\bar{\partial}_{v}$.

Pick a non-negative integer $p \equiv \omega \cdot S-(g-1) \bmod 2(2.20)$, and write

$$
\lambda=\frac{1}{2}((g-1)-p-w) .
$$

Let $J=J_{\lambda}$ be the Jacobian torus parametrizing line bundles of degree $-\lambda$ on $\Sigma$, and let

$$
\mathscr{P}_{\lambda} \rightarrow \Sigma \times J
$$

be the Poincaré bundle: the universal family of holomorphic line bundles of degree $-\lambda$, normalized so that $\mathscr{P}_{\lambda}$ is trivial on $\{\sigma\} \times J$, for some base-point $\sigma \in \Sigma$. For $\jmath \in \mathscr{P}_{\lambda}$, we write $\mathscr{L}_{\jmath}$ for the corresponding bundle of degree $-\lambda$ on $\Sigma$. 
The space $J \times V$ parametrizes a family of bundles $\mathscr{L}_{\jmath}^{-1} \otimes \mathscr{E}_{v}$ on $\Sigma$, and a family of Fredholm operators

$$
\bar{\partial}_{\jmath, v}: \Omega^{0,0}\left(\mathscr{L}_{\jmath}^{-1} \otimes \mathscr{E}_{v}\right) \longrightarrow \Omega^{0,1}\left(\mathscr{L}_{\jmath}^{-1} \otimes \mathscr{E}_{v}\right) .
$$

The numerical index of $\bar{\partial}_{\jmath, v}$ is

$$
\operatorname{ind} \bar{\partial}_{\jmath, v}=-(p+g-1)
$$

by the Riemann-Roch theorem. We consider the subset

$$
\Xi_{V}=\left\{(\jmath, v) \mid \operatorname{dim} \operatorname{Ker} \bar{\partial}_{\jmath, v} \neq 0\right\} \subset J \times V .
$$

We make two assumptions about $\Xi_{V}$ in the calculations below:

Condition 6.8.

(a) that $\operatorname{dim} \operatorname{Ker} \bar{\partial}_{\xi}=1$ for all $\xi=(\jmath, v)$ in $\Xi$, (and so $\operatorname{dim} \operatorname{Coker} \bar{\partial}_{\xi}=$ $p+g)$

(b) that the linearization of the family $\bar{\partial}_{\jmath, v}$ around each $\xi \in \Xi$ is a surjective linear map

$$
\delta_{\xi}: T_{\xi}(J \times V) \rightarrow \operatorname{Hom}\left(\operatorname{Ker} \bar{\partial}_{\xi}, \text { Coker } \bar{\partial}_{\xi}\right) .
$$

In (b), note that $V$ is not supposed to be complex, so $\delta_{\xi}$ is a linear map over $\mathbb{R}$. Its surjectivity is the statement that, in the neighbourhood of $\xi$, the family $\bar{\partial}_{\jmath, v}$ is transverse to the stratum of operators with kernel of dimension 1 inside the space of Fredholm operators of index $-(p+g-1)$.

Under these hypotheses, $\Xi_{V}$ is a smooth submanifold of $J \times V$ of real codimension $2(p+g)$. Its tangent space at $\xi$ is the kernel of $\delta_{\xi}$ and its normal bundle can be oriented using the complex orientation of the image of $\delta_{\xi}$. Using this orientation, $\Xi_{V}$ defines a cohomology class

$$
\text { P.D. }\left[\Xi_{V}\right] \in H^{2 p+2 g}(J \times V) \text {. }
$$

We put

$$
\Pi_{V}=\mathrm{P} . \mathrm{D} \cdot\left[\Xi_{V}\right] /[J] \in H^{2 p}(V),
$$

and set ourselves the task of calculating $\Pi_{V}$. We will restrict our attention to the case

$$
\operatorname{dim} V=2 p .
$$


In this case $\Xi_{V}$ is zero-dimensional, and evaluating $\Pi_{V}$ on $[V]$ gives the number of points in $\Xi_{V}$ counted with sign.

Lemma 6.11. Let $\sigma \in K(J \times V)$ be the index of the family (6.6). Then P.D. $\left[\Xi_{V}\right]=c_{p+g}(-\sigma)$.

Proof. For each point $\xi$ of the finite set $\Xi_{V}$, let $M_{\xi}$ be the kernel of $\bar{\partial}_{\xi}$. By condition (a) above, this is a line, and there is no obstruction to extending $M_{\xi}$ to a trivial line subbundle $M$ over all of $J \times V$ so as to give a decomposition

$$
\Omega^{0,0}\left(\mathscr{L}_{\jmath}^{-1} \otimes \mathscr{E}_{v}\right)=M_{\jmath, v} \oplus H_{\jmath, v}
$$

Let $N$ be the vector bundle of rank $(p+g)$ over $J \times V$ with fibre

$$
N_{\jmath, v}=\Omega^{0,1}\left(\mathscr{L}_{\jmath}^{-1} \otimes \mathscr{E}_{v}\right) / \bar{\partial}\left(H_{\jmath, v}\right)
$$

In this way, the family of Fredholm operators is reduced to a family of finite-dimensional operators, giving a homomorphism of vector bundles

$$
d: M \rightarrow N
$$

Since $M$ is trivial, the index $\sigma$ is $-[N]$ in $K$-theory. The zeros of $d$ are the points of $\Xi_{V}$, and these zeros are transverse by (b) above. The orientation defined for the normal bundle of $\Xi_{V}$ coincides with usual orientation of the normal bundle of the zero-set of $d$. So the Poincaré dual of $\Xi_{V}$ represents the top Chern class of $N$, which is $c_{p+g}(-\sigma)$. q.e.d.

Let $\mathbf{c}$ be the 4-dimensional class

$$
\mathbf{c}=c_{2}(\mathbf{E})-\frac{1}{4} c_{1}(\mathbf{E})^{2} \in H^{4}(\Sigma \times V),
$$

and $\mathbf{w}$ the first Chern class of the universal bundle,

$$
\mathbf{w}=c_{1}(\mathbf{E}) \in H^{2}(\Sigma \times V),
$$

so that

$$
\operatorname{ch}(\mathbf{E})=2 \exp (\mathbf{w} / 2) \cos (\sqrt{\mathbf{c}})
$$

formally. Regarding $x, a_{i}$ and $S$ as a basis for $H_{*}(\Sigma)$, we let $\hat{x}, \hat{a}_{i}$ and $\hat{S}$ be the dual basis of $H^{*}(\Sigma)$, so that $\hat{x}=1$ etc. For $i \leq g$ we have

$$
\hat{a}_{i} \smile \hat{a}_{i+g}=\hat{S} .
$$


We can decompose $\mathbf{c}$ as

$$
\mathbf{c}=\nu(x)+\nu(S) \smile \hat{S}+\sum \nu\left(a_{i}\right) \smile \hat{a}_{i} .
$$

This formula defines $\nu$ (Newstead's map) as a map $H_{i}(\Sigma) \rightarrow H^{4-i}(V)$, which we may extend to a map of algebras

$$
\nu: \mathbb{A}(\Sigma) \rightarrow H^{*}(V)
$$

For brevity, we write

$$
\eta=\sum_{i=1}^{2 g} \nu\left(a_{i}\right) \smile \hat{a}_{i}
$$

A short calculation gives $\eta^{2}=-2 \nu(\Gamma) \hat{S}$ and $\eta^{3}=0$. This allows us to calculate the powers of $\mathrm{c}$ : we have

$$
\mathbf{c}^{s}=\nu\left(x^{s}\right)+s \nu\left(x^{s-1} S\right) \hat{S}+s \nu\left(x^{s-1}\right) \eta-s(s-1) \nu\left(x^{s-2} \Gamma\right) \hat{S}
$$

We record also the Chern character of the Poincaré bundle. We have

$$
c_{1}\left(\mathscr{P}_{\lambda}^{-1}\right)=\lambda \hat{S}+\tau
$$

where

$$
\tau=\sum_{1}^{2 g} \alpha_{i} \smile \hat{a}_{i}
$$

and $\alpha_{i}$ is a standard basis for $H^{1}(J)$. Using the relation

$$
\begin{aligned}
\tau^{2} & =2\left(\sum_{1}^{g} \alpha_{i} \smile \alpha_{i+g}\right) \smile \hat{S} \\
& =-2 \Theta \smile \hat{S}
\end{aligned}
$$

we obtain

$$
\operatorname{ch}\left(\mathscr{P}_{\lambda}^{-1}\right)=1+(\lambda \hat{S}+\tau)-(\Theta \smile \hat{S}),
$$

where $\Theta$ is the standard 2-dimensional class satisfying $\Theta^{g}[J]=g$ !. There is one further class which appears in our calculation, namely the class

$$
\Delta=\sum_{1}^{g}\left(\nu\left(a_{i+g}\right) \alpha_{i}-\nu\left(a_{i}\right) \alpha_{i+g}\right) \in H^{4}(J \times V)
$$


which arises in the formula for the product

$$
\eta \smile \tau=\Delta \smile \hat{S} \in H^{6}(\Sigma \times J \times V) .
$$

Finally, we have

$$
\mathbf{w}=c_{1}(\omega)+\kappa
$$

where $\kappa=c_{1}(K)$ is a 2-dimensional class on $V$. (Recall the assumption concerning $\Lambda^{2} \mathbf{E}$ made at the beginning of this subsection.)

From the index theorem for families, we now obtain

$$
\operatorname{ch}(-\sigma)=-\operatorname{ch}\left(\mathscr{P}_{\lambda}^{-1}\right) \operatorname{ch}(\mathbf{E}) \operatorname{Td}(\Sigma) /[\Sigma] .
$$

Because $\kappa$ is pulled back from $V$, it comes out of the formula and we can write

(6.14) $\exp (-\kappa / 2) \operatorname{ch}(-\sigma)$

$$
\begin{aligned}
= & -(1+(\lambda \hat{S}+\tau)-\Theta \hat{S}) \\
& \times\left(1+c_{1}(\omega) / 2\right)(1-(g-1) \hat{S}) \cos (\sqrt{\mathbf{c}}) \\
= & -\left(1-\frac{1}{2}(g-1+p) \hat{S}+\tau-\Theta \hat{S}\right) \\
& \times\left(\sum(-1)^{s} \mathbf{c}^{s} /(2 s) !\right) .
\end{aligned}
$$

Over $\mathbb{Q}$ we can introduce the element of $K$-theory

$$
\tilde{\sigma}=\sigma \otimes[K]^{-1 / 2} .
$$

Since $-\sigma$ has rank $p+g-1$, we have $c_{p+g}(-\tilde{\sigma})=c_{p+g}(-\sigma)$, and this allows us to ignore $\kappa$ and work with $\tilde{\sigma}$ from now on. Taking the formula (6.13) for the powers of $\mathbf{c}$ and using it in (6.14), together with the formula for $\eta \tau$, we finally obtain

$$
\begin{aligned}
(6.15) \operatorname{ch}_{2 s}(-\tilde{\sigma}) & =\frac{(-1)^{s}}{(2 s) !}\left((p+g-1) \nu\left(x^{s}\right)-2 s \nu\left(x^{s-1}\right) \Delta\right) ; \\
\operatorname{ch}_{2 s+1}(-\tilde{\sigma}) & =\frac{(-1)^{s}}{(2 s+1) !}\left((4 s+2) \nu\left(x^{s}\right) \Theta+\nu\left(x^{s} S\right)-s \nu\left(x^{s-1} \Gamma\right)\right) .
\end{aligned}
$$

To extract a formula for $\Pi_{V}$, we must now derive the Chern class $c_{p+g}(-\tilde{\sigma})$ from these expressions for the Chern character, and then we must integrate over $[J]$. To deal with the latter first, the only classes 
involving the cohomology of $J$ in the above expressions are $\Delta$ and $\Theta$. The integral of a general combination of these two is given by

$$
\Delta^{2 r} \Theta^{g-r} /[J]=(-2)^{r}(g-r) ! r ! \nu\left(\Gamma^{r}\right) \in H^{6 r}(V) .
$$

At this stage it is clear that the formula for $\Pi_{V}$ can be expressed in terms of classes in the image of $\nu$. We therefore have:

Proposition 6.17. There is an element $\pi_{p, g} \in \mathbb{A}(\Sigma)$ of degree $2 p$, expressible as a polynomial in $S, x$ and $\Gamma$ (and as such depending only on $p$ and $g$ ), such that

$$
\Pi_{V}=2^{g} \nu\left(\pi_{p, g}\right) \in H^{2 p}(V)
$$

The factor $2^{g}$ is introduced here for convenience, matching the same factor in (5.9).

Proposition 6.18. For $p \leq 4$ the polynomials $\pi_{p, g}$ are given by:

$$
\begin{aligned}
& \pi_{0, g}=1 \\
& \pi_{1, g}=S \\
& \pi_{2, g}=\frac{1}{2}\left(S^{2}-(g-1) x\right) \\
& \pi_{3, g}=\frac{1}{6}\left(S^{3}-(3 g-4) S x-\Gamma\right) \\
& \pi_{4, g}=\frac{1}{24}\left(S^{4}-2(3 g-5) S^{2} x+3(g-1)(g-3) x^{2}-4 S \Gamma\right) .
\end{aligned}
$$

The coefficient of $S^{p}$ in $\pi_{p, g}$ is $1 / p$ !, for all $p$.

Proof. We begin with the last statement. The $S^{p}$ term in $\pi_{p, g}$ arises from the term with $\nu(S)^{p} \Theta^{g}$ in $c_{p+g}(-\tilde{\sigma})$. We can therefore neglect $x$, $\Delta$ and $\Gamma$, and write $(6.15)$ as

$$
\operatorname{ch}(-\tilde{\sigma})=(p+g-1)+(2 \Theta+\nu(S))
$$

Only $\mathrm{ch}_{0}$ and $\mathrm{ch}_{1}$ have survived. The following identity among symmetric polynomials (see [1] for example) relates the Chern classes $c_{d}$ to the Chern character $\mathrm{ch}_{k}$ :

$$
c_{d}=\sum \frac{1}{h_{1} ! h_{2} ! \cdots h_{j} !} y_{1}^{h_{1}} \cdots y_{j}^{h_{j}}
$$

where

$$
y_{j}=(-1)^{j-1}(j-1) ! \mathrm{ch}_{j}
$$


and the sum is taken over all $h_{i}$ such that the degree of the cohomology class on the right-hand side is $d$. In this first case, the formula reduces to $c_{d}=\left(\mathrm{ch}_{1}\right)^{d} / d$ ! from which we obtain

$$
c_{p+g}=\frac{2^{g}}{p ! g !} \nu(S)^{p} \Theta^{g}
$$

and hence $\Pi_{V}=\left(2^{g} / p !\right) \nu(S)^{p}$, which gives the term $S^{p} / p$ ! in $\pi_{p, g}$ as required.

Next we examine the coefficient of $S^{p-2} x$. For this purpose we can neglect $\Gamma, \Delta$ and $x^{2}$, and write

$$
\operatorname{ch}(-\tilde{\sigma})=(p+g-1)+(2 \Theta+\nu(S))-\frac{1}{2}(p+g-1) \nu(x)-\frac{1}{6}(6 \nu(x) \Theta+\nu(x S)),
$$

or

$$
\begin{aligned}
& y_{1}=2 \Theta+\nu(S) \\
& y_{2}=\frac{1}{2}(p+g-1) \nu(x) \\
& y_{3}=-\frac{1}{3}(6 \nu(x) \Theta+\nu(x S)) .
\end{aligned}
$$

Neglecting terms which cannot contribute $S^{p-2} x$ we have

$$
c_{p+g}=\frac{1}{(p+g-2) !} y_{1}^{p+g-2} y_{2}+\frac{1}{(p+g-3) !} y_{1}^{p+g-3} y_{3},
$$

from which we extract the terms with $\Theta^{g} \nu\left(S^{p-2} x\right)$ :

$$
\begin{gathered}
\left(\frac{2^{g}(p+g-1)}{2 g !(p-2) !}-\frac{2^{g}}{3 g !(p-3) !}-\frac{2^{g}}{(g-1) !(p-2) !}\right) \Theta^{g} \nu\left(S^{p-2} x\right) \\
=\frac{2^{g}}{g !(p-2) !}\left(-\frac{g}{2}+\frac{(p+1)}{6}\right) \Theta^{g} \nu\left(S^{p-2} x\right) .
\end{gathered}
$$

Thus the coefficient of $S^{p-2} x$ in $\pi_{p, g}$ is

$$
\frac{1}{(p-2) !}\left(-\frac{g}{2}+\frac{(p+1)}{6}\right) \text {. }
$$

This gives three more of the non-zero coefficients in the Proposition, and completes the calculation of $\pi_{p, g}$ for $p \leq 2$ (which is all that we need for Theorem 1.7). 
From (6.16) we see that the coefficient of $S^{p-3} \Gamma$ in $\pi_{p, g}$ arises from the terms involving $\nu\left(S^{p-3}\right) \Delta^{2} \Theta^{g-1}$ and $\nu\left(S^{p-3} \Gamma\right) \Theta^{g}$ in $c_{p+g}$. We therefore write

$$
\begin{aligned}
& y_{1}=2 \Theta+\nu(S) \\
& y_{2}=-\Delta \\
& y_{3}=\nu(\Gamma) / 3,
\end{aligned}
$$

and calculate

$$
\begin{aligned}
c_{p+g}= & \frac{1}{2(p+g-4) !} y_{1}^{p+g-4} y_{2}^{2}+\frac{1}{(p+g-3) !} y_{1}^{p+g-3} y_{3} \\
= & \frac{2^{g-1}}{2(g-1) !(p-3) !} \Theta^{g-1} \Delta^{2} \nu\left(S^{p-3}\right) \\
& \quad+\frac{2^{g}}{3 g !(p-3) !} \Theta^{g} \nu\left(S^{p-3} \Gamma\right),
\end{aligned}
$$

ignoring other terms. Applying the formulae (6.16) we obtain

$$
\Pi_{V}=-\frac{2^{g}}{6(p-3) !} \nu\left(S^{p-3} \Gamma\right),
$$

which gives the terms $-\Gamma / 6$ in $\pi_{3, g}$ and $-S \Gamma / 6$ in $\pi_{4, g}$. We leave the coefficient of $x^{2}$ in $\pi_{4, g}$ as an exercise for the reader. q.e.d.

Two small generalizations. Rather than being a smooth manifold, $V$ could be a $2 p$-dimensional simplicial complex. We should assume that the family of Fredholm operators (6.6) is smooth on each topdimensional simplex, that the jumping locus $\Xi_{V}$ lies in $J \times U \subset J \times V$, where $U$ is the union of the interiors of the top-dimensional faces. Orienting the normal bundle of $\Xi_{V}$ as before, we still have a dual cohomology class (in fact a cocycle) and can define $\Pi_{V}$ by (6.10). Given a top-dimensional cycle $[V]$ on $V$, we can evaluate $\Pi_{V}$ on $[V]$ by counting points of $\Xi_{V}$, using the coefficients of $[V]$ as multiplicities.

A second point which arises in practice is that there might not be a universal bundle $\mathbf{E}$. It can happen that there are only local families. That is, $V$ has a finite open cover by open sets $U_{i}$, and over $\Sigma \times U_{i}$ there is a bundle $\mathbf{E}_{i}$ with an isomorphism $\psi_{i}: \Lambda^{2} \mathbf{E}_{i} \rightarrow \omega$. On the overlaps $U_{i} \cap U_{j}$ there will be isomorphisms $\varphi_{i, j}$ respecting the $\psi_{i}$, but on triple overlaps, the cocycle condition will fail by a sign:

$$
\varphi_{i, j} \varphi_{j, k} \varphi_{k, l}= \pm 1
$$


These failures define a $\mathbb{Z} / 2$-valued 2-cocycle and hence a cohomology class $\kappa_{2} \in H^{2}(V ; \mathbb{Z} / 2)$. If this class can be lifted to an integral class $\kappa$, then there will be a universal bundle $\mathbf{E}$ for the family, in the sense described at the beginning of this subsection.

If there is no integral lift $\kappa$, the set $\Xi_{V}$ and its normal orientation can still be defined using the local families $\mathbf{E}_{i}$, and we therefore obtain a class $\Pi_{V} \in H^{2 p}(V)$ as before. On the other hand, we can still define a map $\nu$, over the rationals, using the 4-dimensional class

$$
\mathbf{c}=-\frac{1}{4} p_{1}(\mathbf{P}),
$$

where $\mathbf{P}$ is the (well-defined) $\mathrm{SO}(3, \mathbb{C})$ bundle on $\Sigma \times V$, associated to the $\mathbf{E}_{i}$. This is the class which is equal to $c_{2}(\mathbf{E})-\frac{1}{4} c_{1}(\mathbf{E})^{2}$ in the case that there is an integral lift.

Proposition 6.20. In the case that $\kappa_{2}$ has no integral lift, the statement of Proposition 6.17 continues to hold (with the same polynomials $\pi_{p, g}$ ), with $\nu$ and $\Pi_{V}$ defined as above.

Proof. One way to argue is to show that there is a $2 p$-dimensional complex $W$, a class $[W] \in H^{2 p}(W)$ and a map $f: W \rightarrow V$ such that $f_{*}[W]$ is a non-zero multiple of $[V]$ and such that the pull-back $f^{*}\left(\kappa_{2}\right)$ has an integral lift in $H^{2}(W ; \mathbb{Z})$. The previous calculations can then be applied to the pulled-back family of bundles parametrized by $W$.

In fact, one can arrange that $f^{*}\left(\kappa_{2}\right)$ is zero. To see this, pick a basepoint $\sigma \in \Sigma$, and let $\mathbf{P}_{\sigma}$ be the restriction of $\mathbf{P}$ to $V \times\{\sigma\}$ (which we identify with $V$ ). We must choose $f$ and $W$ so that the $\mathrm{SO}(3)$ bundle $f^{*}\left(\mathbf{P}_{\sigma}\right)$ has a lift to an $\mathrm{SL}(2, \mathbb{C})$ bundle. Let $\mathscr{W}$ be the homotopy quotient of $\mathbf{P}_{\sigma}$ by $\mathrm{SL}(2, \mathbb{C})$. This is a fibre bundle over $V$ with fibre $\mathbb{R} P^{\infty}$, and the pull-back of $\mathbf{P}_{\sigma}$ to $\mathscr{W}$ lifts to $\mathrm{SL}(2, \mathbb{C})$ tautologically. Since the fibre has no rational homology, the map $\mathcal{W} \rightarrow V$ gives an isomorphism on rational homology, and in particular there is a rational cycle $[W]$, supported on some $2 p$-dimensional subcomplex $W \subset \mathscr{W}$, whose image is $[V]$. q.e.d.

(iii) A transversality question. Continuing from the previous subsection, we now let $\mathscr{M}^{s}$ denote the moduli space of all strictly stable, rank 2 holomorphic bundles with fixed determinant omega. If $c_{1}(w) \cdot S$ is odd, there will be a universal family over $\Sigma \times \mathscr{M}^{s}$, but in any case, as noted above, we can still describe a well-defined jumping locus

$$
\Xi \subset J \times \mathscr{M}^{s},
$$


with the same definition as (6.7). We wish to investigate the extent to which the transversality conditions (6.8)(a) and (b) hold in this case. Let us decompose $\Xi$ into two subsets: we write

$$
\Xi=\Xi^{i} \cup \Xi^{z}
$$

where $\Xi^{i}$ is the set of $(\jmath, v)$ such that all non-zero maps $\mathscr{L}_{\jmath} \rightarrow \mathscr{E}_{v}$ are injective. The complement $\Xi^{z}$ is the set where at least one such map has a zero.

Lemma 6.21. The transversality conditions (6.8) hold at all points of $\Xi^{i}$.

Proof. If there is are two linearly independent, injective maps $s_{1}, s_{2}$ from $\mathscr{L}_{3}$ to $\mathscr{E}_{v}$ then there is a point $\sigma \in \Sigma$ such that $\left(s_{1}\right)_{\sigma}$ and $\left(s_{2}\right)_{\sigma}$ are dependent, for otherwise $\mathscr{E}_{v}$ is the direct sum of the images of the two maps and therefore not stable. So some linear combination of $s_{1}$ and $s_{2}$ is a map with a zero, showing that $(\jmath, v)$ is in $\Xi^{z}$. Thus (a) holds on $\Xi^{i}$.

Now we show that (a) implies (b). Since we are concerned with the local properties near $(\jmath, v)$, we can exploit the fact that there is a local isomorphism between $J \times \mathscr{M}^{s}$ and $\mathscr{M}^{+}$, where $\mathscr{M}^{+}$is the moduli space of stable rank 2 bundles of degree $\omega \cdot S+2 \lambda$, without fixed determinant: the local isomorphism sends $(\mathscr{L}, \mathscr{E})$ to $\mathscr{L}^{-1} \otimes \mathscr{E}$. So we consider a rank 2 bundle $\mathscr{E}$ with $H^{0}(\mathscr{E})$ of dimension 1 , spanned by a section $s$. The map of the tangent space in (b) corresponds now to the map

$$
H^{1}(\text { End } \mathscr{E}) \longrightarrow \operatorname{Hom}\left(H^{0}(\mathscr{E}), H^{1}(\mathscr{E})\right)
$$

Since $s$ spans $H^{0}(\mathscr{E})$, we can identify the right-hand side with $H^{1}(\mathscr{E})$, in which case the map is the one induced on $H^{1}$ by the map of sheaves

$$
\operatorname{End}(\mathscr{E}) \stackrel{s^{*}}{\rightarrow} \mathscr{E}
$$

Since $\Sigma$ is one-dimensional, the map is onto. q.e.d.

Let us temporarily record the dependence of $\Xi$ on the integer $p$ by writing $\Xi(p)$. (The integer $p$ enters in determining $\lambda$ and hence the particular component $J$ of the Picard group.)

Lemma 6.22. The bad set $\Xi^{z}(p)$ is contained in the image of an analytic map from $\Sigma \times \Xi(p+2)$.

Proof. If there is a map from $\mathscr{L}$ to $\mathscr{E}$ which as a zero at $\sigma$, then there is a line bundle $\mathscr{L}^{\prime}=\mathscr{L}[\sigma]$ of degree one greater and a non-zero 
map from $\mathscr{L}^{\prime}$ to $\mathscr{E}$. The pair $(\mathscr{L}, \mathscr{E})$ can be recovered from the data $\left(\sigma, \mathscr{L}^{\prime}, \mathscr{E}\right)$. q.e.d.

The next proposition summarizes our conclusions:

Proposition 6.23. For $p<g-2$, the analytic subset $\Xi \subset J \times \mathscr{M}^{s}$ has complex codimension $p+g$, and at a generic point $(\jmath, v)$ there is a unique, injective map from $\mathscr{L}_{\jmath}$ to $\mathscr{E}_{v}$. The class

$$
\Pi=\mathrm{P} . \mathrm{D} \cdot[\Xi] /[J] \in H^{2 p}\left(\mathscr{M}^{s} ; \mathbb{Q}\right)
$$

is given in terms of Newstead's classes by an expression

$$
\Pi=2^{g} \nu\left(\pi_{p, g}\right)
$$

where $\pi_{p, g} \in \mathbb{A}(\Sigma)$ are the polynomials which are partially computed in Proposition 6.18.

Proof. At the injective points $\Xi^{i}$, the variety $\Xi$ is smooth and of codimension $p+g$ by Lemma 6.21 , because the transversality conditions ensure this. Using Lemma 6.22, one can see by induction on $p$ that the remaining points $\Xi^{z}$ have codimension at least $p+g+1$. If $\Xi^{i}$ is nonempty, then the class $\Pi$ can be defined. A homology class in $H_{2 p}\left(\mathscr{M}^{s}\right)$ can be represented as a $C^{\infty}$ singular cycle $V \rightarrow \mathscr{M}^{s}$ which is transverse to $\Xi^{i}$ and does not meet $\Xi^{z}$; applying the analysis of subsection (ii) above to the induced family of bundles over $V$, one can then deduce the value of the pairing between $\Pi$ and $[V]$, and so conclude that $\Pi$ has the value claimed for it, as a rational class. (In case $c_{1}(\omega)$ is even and there is no universal bundle, one must use Proposition 6.20.)

If $\Xi^{i}$ is empty then the same reasoning shows that $\nu\left(\pi_{p, g}\right)$ is zero in $H^{2 p}\left(\mathscr{M}^{s}\right)$. The polynomial $\pi_{p, g}$ is non-trivial because of the last clause of (6.18), so this is a non-trivial relation among the Newstead generators. This is not possible if $p<g-2$ ( $c f$ Proposition 5.2). q.e.d.

(iv) Bundles with parabolic structure. Let $W$ be the ruled surface containing $\Sigma_{+}$and $\Sigma_{-}$, as at the beginning of section 3 (ii). Let $W^{o}$ be again the Riemannian manifold obtained by removing $\Sigma_{-}$ from $W$ and equipping the complement with a metric with cylindrical end. We also want to arrange that the metric has a cone-like singularity along $\Sigma_{+}$, with small cone-angle (see the discussion in section $2(\mathrm{v})$ ). Let $E^{w}$ be a $\mathrm{U}(2)$ bundle on $\Sigma_{+}$with determinant $w$, and let it be pulled back to $W^{o}$. Let $\mathscr{R}_{\mathrm{ev}}^{w}$ be the corresponding representation variety for 
the restriction of this bundle to the three-manifold $Y$, and consider again the moduli space

$$
M^{p}\left(W^{o}\right):=M_{0}^{w, p}\left(W^{o}, \Sigma_{+} ; \mathscr{R}_{\mathrm{ev}}^{w, s}\right)
$$

of singular connections with holonomy $\alpha$ at $\Sigma_{+}$, asymptotic to the smooth part $\mathscr{R}_{\mathrm{ev}}^{w, s}$. The dimension of this moduli space is

$$
\operatorname{dim} M^{p}\left(W^{o}\right)=(6 g-6)-2 p
$$

and the connections $A$ in this moduli space have Chern-Weil integral

$$
c(A)=2 \alpha l-\alpha^{2} n,
$$

where $p=(g-1)-2 l$ and $n=Q\left(\Sigma_{+}\right)$as usual. If $p$ is small compared to $n$ and $g$, so that $2 p \leq \Delta$ (where $\Delta$ is as in (4.26)), and if $\alpha$ is sufficiently small (see 4.24 ), then the end-limit map

$$
r: M^{p}\left(W^{o}\right) \rightarrow \mathscr{R}_{\mathrm{ev}}^{w, s}
$$

defines a class

$$
\Omega=\left[M^{p}\left(W^{o}\right), r\right] \in H^{2 p}\left(\mathscr{R}_{\mathrm{ev}}^{w, s}\right)
$$

in the sense of Definition 4.8. This was shown in Theorem 4.28. By definition (see Proposition 5.9), the element $\omega_{p, 0}^{w}$ is the unique element of $\mathbb{A}(\Sigma)$ which is homogeneous of degree $2 p$ and satisfies

$$
\Omega=2^{g} \nu\left(\omega_{p, 0}^{w}\right),
$$

where $\nu$ is the Newstead map $\mathbb{A}(\Sigma) \rightarrow H^{*}\left(\mathscr{R}_{\mathrm{ev}}^{w, s}\right)$. After choosing a holomorphic structure $\omega$ for the line bundle $w$, we identify $\mathscr{R}^{w, s}$ with the moduli space of stable bundles $\mathscr{M}^{s}$.

At the beginning of this section, we laid out the aim of a partial calculation of the polynomials $\omega_{p, 0}^{w}$. In view of the calculations of the polynomials in part (ii) above, what we need to do is to show that $\omega_{p, 0}^{w}$ is equal to the polynomial $\pi_{p, g}$. The Propositions 6.1-6.4 will then follow. Because of Proposition 6.23, what we need to establish is therefore:

Proposition 6.25. The class $\Omega$ defined by the moduli space $M^{p}\left(W^{o}\right)$ in equation $(6.24)$ is equal to the class $\Pi=[\Xi] /[J]$ which appears in (6.23). 
The good part, $\Xi^{i} \subset \Xi$, defined above, is a smooth manifold, and using Definition (4.8) we can describe the class $\Pi$ as

$$
\Pi=\left[\Xi^{i}, p_{2}\right],
$$

where $p_{2}: J \times \mathscr{M}^{s} \rightarrow \mathscr{M}^{s}$ is the projection on the second factor. A direct approach to the proof of Proposition 6.25 would be to show that, for a suitable choice of metric, the moduli space $M^{p}\left(W^{o}\right)$ could be identified with $\Xi^{i}$ (give or take some strata of codimension 2 or more), so that $r$ coincided with $p_{2}$. We believe this can be done by extending the results of [20], but we prefer to adopt instead the line taken in sections 7 and 9 of [27], which means first showing that $\Omega$ can be described in terms of gauge theory on the closed manifold $W$ rather than the cylindrical end space $W^{\circ}$. The reader should refer to [27] for more details of the argument we present here.

Let the closed ruled surface $W$ be equipped with a Riemannian metric $g_{R}$ by taking the given metric on $W^{\circ}$, truncating the cylindrical end at distance $R$ and attaching the tubular neighbourhood of $\Sigma_{-}$with some fixed, compatible metric. Consider the moduli space

$$
M^{p}(W):=M_{0}^{w, p, \alpha}\left(W, \Sigma_{+}\right) .
$$

According to Lemma 7.5 of [27], if $R$ is large and $\alpha$ is small, the only non-compactness phenomenon which occurs for a sequence of connections in this moduli space is that curvature can concentrate on $\Sigma_{+}$, leading to a different, larger value of $p$ (or smaller monopole number $l$ ) in the weak limit. We can therefore define a compactification

$$
\bar{M}^{p}(W)=M^{p}(W) \cup M^{p+2}(W) \cup \cdots .
$$

The moduli spaces $M^{p^{\prime}}(W)$ are free of reducibles and are empty once $2 p^{\prime} \geq(g-1)$ (Lemma 7.2 of [27]). Because no bubbling off occurs at $\Sigma_{-}$, restriction to $\Sigma_{-}$defines a continuous map

$$
\bar{\rho}: \bar{M}^{p}(W) \rightarrow \mathscr{B}_{\Sigma_{-}}^{w} .
$$

The proof of Lemma 7.6 of [27] shows that the connections $\bar{\rho}(A)$ on

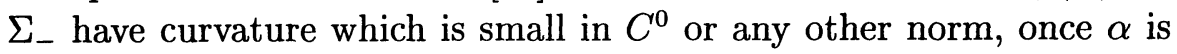
small. We can therefore take it that the image of $\bar{\rho}$ is contained in a 
neighbourhood $\mathscr{U}$ of the moduli space of flat connections $M^{\text {flat }} \subset \mathscr{B}^{w}$ with the property that the heat-flow defines a retraction

$$
h: \mathscr{U} \rightarrow M^{\text {flat }} .
$$

If we identify $M^{\text {flat }}$ with $\mathscr{M}$, the moduli space of semi-stable bundles, then the composite $h \circ \bar{\rho}$ has another description: it is the map which assigns to $A$ the $s$-equivalence class of the semi-stable bundle $\mathscr{E}_{A} \rightarrow \Sigma_{-}$ defined by restricting $A$ to $\Sigma_{-}$. Let $M^{p}(W)^{s}$ denote the part of $M^{p}(W)$ which lies over $\mathscr{M}^{s} \subset \mathscr{M}$, and consider the class dual to the image of $M^{p}(W)^{s}$ in $\mathscr{M}^{s}$ :

$$
\Omega^{\prime}=\left[M^{p}(W)^{s}, h \circ \rho\right] \in H^{2 p}\left(\mathscr{M}^{s}\right) .
$$

Lemma 6.27. The class $\Omega^{\prime}$ defined by the moduli space on the closed ruled surface $W$ is equal to the class $\Omega$ of (6.24) defined by the moduli space of the cylindrical end manifold $W^{o}$, as long as $R$ is large and $\alpha$ is small.

Proof. Let $K$ be any compact subset of $\mathscr{M}^{s}$, and let $M^{p}(W)_{K}$ and $M^{p}\left(W^{o}\right)_{K}$ be the parts of the two moduli spaces which lie over $K$. The connections in $M^{p}\left(W^{o}\right)_{K}$ have curvature which is uniformly small on the end of the manifold, so by gluing these connections to the flat connections on the neighbourhood of $\Sigma_{-}$we obtain a map

$$
\Psi: M^{p}\left(W^{o}\right)_{K} \rightarrow M^{p}(W),
$$

which is a diffeomorphism onto its image when $R$ is large. This can be extended to continuous a map

$$
\bar{\Psi}: \bar{M}^{p}\left(W^{o}\right)_{K} \rightarrow \bar{M}^{p}(W) .
$$

We have also continuous maps

$$
\begin{aligned}
& \bar{r}: \bar{M}^{p}\left(W^{o}\right) \rightarrow \mathscr{R}_{\mathrm{ev}}^{w, s} \cong M^{\text {flat }} \\
& \bar{\rho}: \bar{M}^{p}(W) \rightarrow \mathscr{U} .
\end{aligned}
$$

The composite $\bar{\rho} \bar{\Psi}$ is $C^{0}$ close to $\bar{r}$ in $\mathscr{B}_{\Sigma_{-}}$, and is $C^{1}$ close on the smooth stratum $M^{p}\left(W^{o}\right)_{K}$. Composing with the retraction $h$, we have a map $h \bar{\rho} \bar{\Psi}$ which is still $C^{1}$ close to $\bar{r}$. It follows that if we evaluate $\Omega^{\prime}$ and $\Omega$ on a closed $2 p$ chain $V$ in $\mathscr{M}^{s}$ by counting points of intersection, we will obtain the same count, because the two point-sets will 
be isomorphic. (A more detailed version of this argument is given in section 7 of [27].) q.e.d.

The definition of $\Omega^{\prime}$ did not rely on the specific nature of the metric $g_{R}$ on $W$. Two things about the metric are all that is important. First, the metric should be such that there are no reducible connections in the moduli space $M^{p}(W)$. This is a condition that depends on $\alpha$ : since $b^{+}(W)=1$, there is a one-dimensional space of self-dual harmonic forms, which we can take to be spanned by the Poincaré dual of the class

$$
\left[\Sigma_{+}\right]+t[F]
$$

where $F$ is the 2-sphere fibre of the ruled surface and $t \in \mathbb{R}$; there are no reducibles in $M^{p}(W)$ provided that

$$
\alpha(n+t)<1
$$

in the case that $w$ is even, or provided that

$$
\alpha(n+t)<\frac{1}{2}
$$

in the odd case. (The former statement is proved in section 7 of [27], and the reader can easily adapt the argument to the odd case.) The other feature of the metric which is important is its geometry in the neighbourhood of $\Sigma_{-}$, because this determines how small $\alpha$ will need to be in order to ensure that the image of $\bar{\rho}$ lands in $\mathcal{U}$. If $\alpha$ and the Riemannian metric are allowed to vary in a 1-parameter family, the class $\Omega^{\prime}$ remains well-defined and unchanged, as long as 6.29 is true at all points along the path and the geometry near $\Sigma_{-}$is constant.

As in [27] therefore, we are free to calculate $\Omega^{\prime}$ using a suitable Kähler metric. As was shown in Appendix 2 of [27], we can choose a Kähler metric $\varphi$ on $W$ with a cone-like singularity along $\Sigma_{+}$, whose Kähler class $[\varphi]$ has the form 6.28 for some small, positive $t$. We summarize, from [27], a description of the moduli space in this situation.

Proposition 6.30. Let $\varphi$ be a Kähler metric on $W$, as above. Suppose that the cone-angle in the metric is $2 \pi / \nu$ for some integer $\nu$, and that $\alpha$ is rational with denominator $\nu$. Suppose that $\alpha(n+t)$ is less than 1 in the even case, or less than $\frac{1}{2}$ in the odd case. Then the 
moduli space $M^{p}(W)$ is regular for all $p$ and can be identified with the space of isomorphism classes of triples $(\mathscr{E}, \psi, \mathscr{L})$, where

(a) $\mathscr{E}$ is a semi-stable rank-2 holomorphic bundle on $\Sigma$ with an isomorphism $\psi: \Lambda^{2} \mathscr{E} \rightarrow \omega$; and

(b) $\mathscr{L}$ is a line-subbundle of $\mathscr{E}$ of degree $-\lambda$, where

$$
\lambda=\frac{1}{2}(g-1-\omega \cdot S-p) .
$$

Under this correspondence, the bundle $\mathscr{E}$ associated with a connection $A$ is the same as the holomorphic bundle on $\Sigma_{-}$determined by the restriction of $A$.

Proof. The results of section 8 and section 9(ii) of [27] established this in the case $\omega$ is trivial. The general case presents no new difficulties. q.e.d.

On account of the last clause, the map

$$
h \circ \bar{\rho}: M^{p}(W) \rightarrow \mathscr{M}
$$

corresponds to the map which assigns to $(\mathscr{E}, \psi, \mathscr{L})$ the $s$-equivalence class of $\mathscr{E}$; and the subset $M^{p}(W)^{s} \subset M^{p}(W)$ corresponds to the triples $(\mathscr{E}, \psi, \mathscr{L})$ where $\mathscr{E}$ is strictly stable. Let $M^{p}(W)^{u}$ denote the still smaller set where $\mathscr{E}$ is stable and the line subbundle $\mathscr{L}$ admits just one map to $\mathscr{E}$ (up to a scalar multiple). The proofs of Lemmas 6.21 and 6.22 show that $M^{p}(W)^{u}$ can be identified with $\Xi^{i} \subset J \times \mathscr{M}^{s}$, and also show that the complement of $M^{p}(W)^{u}$ in $M^{p}(W)^{s}$ has real codimension 2 or more. We therefore have equalities

$$
\begin{aligned}
\Omega & =\Omega^{\prime} \\
& =\left[M^{p}(W)^{s}, h \circ \bar{\rho}\right] \\
& =\left[M^{p}(W)^{u}, h \circ \bar{\rho}\right] \\
& =\left[\Xi^{i}, p_{2}\right] \\
& =\Pi .
\end{aligned}
$$

This proves Proposition 6.25, and completes the proofs of Propositions 6.2-6.4. q.e.d.

A word on orientations. The careful reader will note that the arguments above do not make any mention of the standard orientations of the moduli spaces concerned, and can only be taken as calculating $\omega_{p, 0}^{w}$ up to an overall factor of \pm 1 . One way to see that the final sign 
is correct (and that the leading coefficient of $\omega_{p, 0}^{w}$ is not $-1 / p$ !) is to observe that what has been used at every stage in the calculations is the complex orientation of moduli spaces. So, in the case that $(X, \Sigma)$ is a Kähler pair, if we define modified invariants $\tilde{D}_{X}^{w}$ and $\tilde{D}_{X, \Sigma}^{w, p}$ using the complex orientation, the polynomials $\omega_{p}^{w}$ we have partially calculated are the right ones to describe a universal formula for the latter in terms of the former. On the other hand, from [9] one knows that

$$
\tilde{D}_{X}^{w}=(-1)^{\left(w^{2}+K_{X} \cdot w\right) / 2} D_{X}^{w}
$$

when $X$ is given a standard homology orientation determined by the Kähler structure; and in Appendix 1 of [27] it was verified that exactly the same relationship holds between $\tilde{D}_{X, \Sigma}^{w, p}$ and $D_{X, \Sigma}^{w, p}$. Therefore, the overall sign of $\omega_{p}^{w}$ can be correctly calculated by working with the complex orientations throughout. See the Appendix.

\section{The structure theorem}

In this section, $X$ will always be an admissible 4-manifold (see Definition 2.1), and $\Sigma$ will be an oriented embedded surface. As in previous sections, we will write $S$ for the class $[\Sigma]$ in $H_{2}(X)$ and $x$ for the point class. We write $\imath$ for the inclusion of $\Sigma$, and $\jmath$ for the inclusion of $X / \Sigma$ in $X$. From part (ii) onwards, $X$ will always have $b^{1}(X)=0$. In this case, if $\lambda$ is any non-trivial line bundle, one has $b^{+}(\lambda)=b^{+}(X)+b^{1}(\lambda)+1 \geq b^{1}(\lambda)+4$, so the pair $(X, \Sigma)$ is automatically admissible in the sense of Definition 2.23 , and the cylindrical-end manifold $X^{o}$ is admissible in the sense of Definition 2.15.

(i) Relations among invariants. The universal formulae (5.10), expressing the invariants of a pair in terms of the ordinary invariants, give rise to relations among the latter. Recall from [27],[28],[25] that there is a natural identification of moduli spaces of singular connections,

$$
\Phi: M_{k, l}^{w, \alpha} \rightarrow M_{k^{\prime}, l^{\prime}}^{w^{\prime}, \alpha^{\prime}}
$$

in which the holonomy parameter, instanton number, monopole num- 
ber and determinant are related by

$$
\begin{aligned}
\alpha^{\prime} & =\frac{1}{2}-\alpha \\
k^{\prime} & =k+l-\frac{1}{4} Q(\Sigma) \\
l^{\prime} & =\frac{1}{2} Q(\Sigma)-l \\
w^{\prime} & =w-[\Sigma] .
\end{aligned}
$$

As $\mathrm{SO}(3)$ connections on $X / \Sigma$, one has $\Phi[A]=[A]$, and the transformation $\Phi$ just corresponds to changing the preferred orientation of the 2-plane $K$ in the decomposition $\mathfrak{g}_{E}=\mathbb{R} \oplus K$ near $\Sigma$ (see section $2(\mathrm{v})$ ). In terms of the parameters $w$ and $p$ by which we label the invariants $D_{X, \Sigma}^{w, p}$, the transformation is

$$
\begin{aligned}
w^{\prime} & =w-[\Sigma] \\
p^{\prime} & =a(\Sigma)-p
\end{aligned}
$$

where

$$
a(\Sigma)=2 g-2-Q(\Sigma)
$$

as in 1.11. In the Appendix, we shall show that $\Phi$ preserves or reverses the standard orientation of the moduli spaces according to the sign of $(-1)^{p^{\prime}}$.

Remark. (i) The effect of $\Phi$ on the standard orientation was treated in [27] in the case that $w=0$ and $[\Sigma]$ is divisible by 2 . Note that in this case, although $w^{\prime} \equiv 0 \bmod 2$, the value of $w^{\prime}$ itself is used to determine the orientation of the moduli space on the right-hand-side. In this way, our present conventions differ from those of [27] and [25], where the moduli space was always oriented as an $\mathrm{SU}(2)$ moduli space.

(ii) The transformation $w \mapsto w^{\prime}$ does not have order 2 . It would have been possible to define $\Phi$ instead with $w^{\prime}=[\Sigma]-w$, but the formula for the sign change would then have been more complicated: there is an advantage in having the change in $w$ be local to the neighbourhood of $\Sigma$.

The map $\Phi$ respects the process of cutting down moduli spaces by classes $\mu(z)$ for $z \in \jmath_{*} \mathbb{A}(X / \Sigma)$ (where $\jmath: X / \Sigma \rightarrow X$ is again the inclusion), because the chosen geometrical representatives can be made to depend only on the restriction of the connections (as $\mathrm{SO}(3)$ connections) to suitable neighbourhoods $U$ of classes supported in $X / \Sigma$. It follows that

$$
D^{w^{\prime}, p^{\prime}}(z)=(-1)^{p^{\prime}} D^{w, p}(z)
$$


for all $z \in \mathbb{A}(X / \Sigma)$. From this relation, we obtain the following result, which is a central tool in our argument. Recall from (4.26) the value $\Delta$, which is the largest (co)dimension in which our Floer homology theory was valid:

$$
\Delta(n, g)=\min \{2 g-4-(n / 2), 2 n-2\} .
$$

Theorem 7.4. Let $(X, \Sigma)$ be an admissible pair. Let $\imath: \Sigma \rightarrow X$ be the inclusion and $S \in H_{2}(X ; \mathbb{Z})$ the class carried by $\Sigma$. Suppose $Q(S)=n>0$ and suppose the genus $g$ of $\Sigma$ is at least 2 . Let $p$ and $w$ satisfy the parity condition $p \equiv(g-1)-w \cdot S \bmod 2(2.20)$, let $p^{\prime}=a(\Sigma)-p$, and suppose that

$$
\max \left\{p, p^{\prime}\right\} \leq \frac{1}{2} \Delta(n, g) .
$$

Then there is a relation

$$
D^{w}\left(\imath_{*}\left(\omega_{p}^{w}\right) \jmath_{*}(z)\right)=(-1)^{p^{\prime}} D^{w^{\prime}}\left(\iota_{*}\left(\omega_{p^{\prime}}^{w^{\prime}}\right) \jmath_{*}(z)\right),
$$

valid for all $z \in \mathbb{A}(X / \Sigma)$. The elements $\omega_{p}^{w}$ on the left-hand-side are the distinguished (generally inhomogeneous) elements of $\mathbb{A}(\Sigma)$ determined by 5.9, depending only on $Q(\Sigma)$, genus $(\Sigma)$ and $w \cdot \Sigma \bmod 2$. On the right-hand-side, $\omega_{p^{\prime}}^{w^{\prime}}$ is the element corresponding to $w^{\prime}=w-[\Sigma]$ in place of $w$. If either $p$ or $p^{\prime}$ is negative, the corresponding side of the equation is to be interpreted as zero. The $\omega_{p}^{w}$ do not depend on $X$ or $z$.

Proof. This follows immediately from Theorem 5.10 and the relation (7.2). q.e.d.

We describe explicitly the simplest consequences of these relations. Because the concept will occur regularly in this section and the next, we introduce a term to describe surfaces $\Sigma \subset X$ with $a(\Sigma)=0$ :

Definition 7.5. A surface $\Sigma \subset X$ is tight if $a(\Sigma)=0$; that is, if $2 g-2=Q(\Sigma)$.

From this point on, we will frequently abuse notation by omitting $\imath_{*}$ and $\jmath_{*}$ when referring to the images of $\mathbb{A}(\Sigma)$ and $\mathbb{A}(X / \Sigma)$ in $\mathbb{A}(X)$.

Lemma 7.6. Let $X$ be an homology-oriented 4-manifold with $b^{+}-b^{1}$ odd and $b^{+}>1$. Let $\imath: \Sigma \rightarrow X$ be a tight surface representing a class $S$, let $w$ be a line bundle on $X$ and let $w^{\prime}=w-[\Sigma]$. Then the following relations hold.

(a) Suppose $w \cdot \Sigma=\operatorname{genus}(\Sigma)-1 \bmod 2$ and suppose that $\operatorname{genus}(\Sigma) \geq 3$ (or equivalently $Q(S) \geq 4$ ). Then for all $z \in$ 
$\mathbb{A}(X / \Sigma)$, we have

$$
D^{w}(z)=D^{w^{\prime}}(z) \text {. }
$$

(b) Suppose $w \cdot \Sigma=$ genus $(\Sigma)-1 \bmod 2$ and suppose that genus $(\Sigma) \geq 7$ (or equivalently $Q(S) \geq 12$ ). Then for all $z \in$ $\mathbb{A}(X / \Sigma)$, we have

$$
D^{w}\left(S^{2} z\right)-(g-1) D^{w}(x z)=0
$$

where, as usual, $x$ denotes the positive generator of $H_{0}(X ; \mathbb{Z})$.

(c) Suppose $w \cdot \Sigma=$ genus $(\Sigma) \bmod 2$ and suppose that genus $(\Sigma) \geq 5$ (or equivalently $Q(S) \geq 8$ ). Then for all $z \in \mathbb{A}(X / \Sigma)$, we have

$$
D^{w}\left(S^{2} z\right)-g D^{w}(x z)=2 D^{w^{\prime}}(z) .
$$

Proof. (a) Since $a(\Sigma)=0$, we have $p^{\prime}=-p$. The inequality for $g=$ genus $(\Sigma)$ in $(a)$ ensures that the upper bound on $p$ in Theorem 7.4 is satisfied by $p=p^{\prime}=0$, and the parity condition on $w$ means that $p=0$ satisfies the parity condition. We therefore have a relation

$$
D^{w}\left(\omega_{0}^{w} z\right)=D^{w^{\prime}}\left(\omega_{0}^{w^{\prime}} z\right)
$$

The result then follows from Proposition 6.2(a), which says that $\omega_{0}^{w}=\omega_{0}^{w^{\prime}}=1$.

(b) Now take $p=2$ and $p^{\prime}=-2$, so that the right-hand-side of the relation in 7.4 is zero. If $g \geq 7$ then $p$ is in the admissible range, so we have

$$
D^{w}\left(\omega_{2}^{w} z\right)=0 \text {. }
$$

According to Proposition 6.2(c), we have $\omega_{2}^{w}=\frac{1}{2}\left(S^{2}-(g-1) x\right)$, so the result follows.

(c) For this example, we apply the relations using a surface different from $\Sigma$. Let $T^{2}$ be a standard, null-homologous torus in $X / \Sigma$ and let $\tilde{\Sigma}$ be the internal connected sum $\Sigma \# T^{2}$ of genus $\tilde{g}=g+1$. This surface has $a(\tilde{\Sigma})=2$, so we have $p^{\prime}=2-p$. The lower bound on the genus, and the parity condition on $w$, ensures that we can apply the Theorem to the pair $(X, \tilde{\Sigma})$ with $p=2$. This gives a relation

$$
D^{w}\left(\omega_{2}^{w} z\right)=D^{w^{\prime}}\left(\omega_{0}^{w^{\prime}} z\right)
$$


Now $\omega_{0}^{w^{\prime}}=1$ and, as in $(b)$,

$$
\begin{aligned}
\omega_{2}^{w} & =\frac{1}{2}\left(S^{2}-(\tilde{g}-1) x\right) \\
& =\frac{1}{2}\left(S^{2}-g x\right) .
\end{aligned}
$$

The result follows. q.e.d.

(ii) The invariants for $\boldsymbol{K 3}$. We begin with an elementary lemma about embedded surfaces.

Lemma 7.7. If $S \in H_{2}(X ; \mathbb{Z})$ is a homology class with $Q(S) \geq 0$ and $\Sigma_{S}$ is a surface of genus at least 1 representing $S$, then for all $m>0$, the class $m S$ can be represented by an embedded surface $\Sigma_{m S}$ with

$$
a\left(\Sigma_{m S}\right)=\operatorname{ma}\left(\Sigma_{S}\right)
$$

In particular, if $S$ is represented by a tight surface then $m S$ can be represented by a tight surface.

Proof. Let $N$ be a disk-bundle neighbourhood of $\Sigma_{S}$, and consider first the case that its degree $Q(S)$ is strictly positive. Choose $m$ sections $s_{i}: \Sigma_{S} \rightarrow N$, and let $T_{i}$ be their images, $(i=1, \ldots, m)$. These can be chosen so the intersection points $T_{i} \cap T_{j}$ are transverse and have positive sign. Smooth out each intersection point by replacing each pair of intersecting disks with a standard annulus having the same boundary. Thus modified, the union of the $T_{i}$ becomes a smooth embedded surface $\Sigma_{m S}$, and the formula for $a\left(\Sigma_{m S}\right)$ is easily verified.

When $Q(S)$ is zero, the disk bundle $N$ is trivial. As the genus is nonzero, one can choose a surjective homomorphism $\psi: \pi_{1}\left(\Sigma_{S}\right) \rightarrow \mathbb{Z} / m \mathbb{Z}$. Let $P$ be the corresponding principal $\mathbb{Z} / m \mathbb{Z}$ bundle. The circle bundle associated to $P$ is trivial, so $P$ may be embedded in $S^{1} \times \Sigma_{S}$ and hence inside $N$, where its image is the required surface. Note that the surjectivity of $\psi$ ensures that $P$ is connected. q.e.d.

Lemma 7.8. Let $X$ be a K3 surface. Then every homology class $S \in H_{2}(X ; \mathbb{Z})$ with $Q(S) \geq-2$ can be represented by a tight surface.

Proof. Because of the previous Lemma, it is sufficient to consider a primitive class $S$. Further, because the diffeomorphism group of $K 3$ acts transitively on the primitive classes of any given square, we need only construct one tight, embedded, primitive surface for each even self-intersection number $n \geq-2$ (the intersection form takes only even values). The standard Kummer model for $K 3$ shows how $X$ can be realized as an elliptic surface having a section $T$ which is a sphere of 
square -2 . Let $\Sigma^{\prime}$ be the union of $T$ and $(n / 2)+1$ torus fibres, and let $\Sigma$ be the surface obtained by smoothing the intersection points between the tori and $T$ as in the previous Lemma. The genus of $\Sigma$ is $(n / 2)+1$ and its self-intersection number is $n$, so $\Sigma$ is tight. Furthermore, $\Sigma$ realizes a primitive class, because its intersection number with the torus fibre is $1 . \quad$ q.e.d.

The following Lemma is well known, but the sign involved is less familiar than the absolute value of the invariant.

Lemma 7.9. On K3 with its standard homology-orientation, we have

$$
D^{w}(1)= \begin{cases}-1 & \text { if } w^{2}=2 \bmod 4, \\ 0 & \text { if } w^{2}=0 \bmod 4 .\end{cases}
$$

Proof. The second case is merely a consequence of our definitions, for there is no zero-dimensional moduli space when $w^{2} \equiv 0$. The calculation in the first case appeared first in [10] where it was shown that the invariant is 1 for a particular $w$ if the zero-dimensional moduli space is oriented with its 'complex' orientation. According to [9], the orientation determined by $w$ differs from the complex orientation by the $\operatorname{sign}(-1)^{\left(w^{2}+K_{X} \cdot w\right) / 2}$, which is -1 , since $K_{X}=0$. The result for this particular $w$ follows, and as usual one can use the transitivity of the action of the diffeomorphism group to obtain the result for general $w$. q.e.d.

The next series of lemmas determines the entire invariant for $K 3$. The final result is again well known [37],[17], but we include a proof here to show how it can be deduced from the universal relations of the previous subsection together with Lemmas 7.8 and 7.9 and knowledge of the homotopy type and diffeomorphisms of $K 3$. (In particular, the following argument makes no use of Floer homology for homology 3spheres.)

Lemma 7.10. Let $X$ be a $K 3$ with its standard homology-orientation, and let $w$ be any line bundle. Then there is a $w^{\prime}$ with $\left(w^{\prime}\right)^{2}=$ $w^{2}+2 \bmod 4$ such that

$$
D^{w}(x)=-2 D^{w^{\prime}}(1) .
$$

Proof. Let $\Sigma$ be a tight surface representing a class $S$ which is orthogonal to $w$. We can arrange that $Q(S)$ is large and equal to 2 
mod 4. The genus of $\Sigma$ is then even, since $2 g-2=Q(S)$. We can therefore apply Proposition 7.6(c) with $z=1$ to obtain

$$
D^{w}\left(S^{2}\right)-\left(1+\frac{1}{2} Q(S)\right) D^{w}(x)=2 D^{w^{\prime}}(1) .
$$

Now let $\Sigma_{2}$ be a tight surface representing $2 S$. This has odd genus (since genus $\left(\Sigma_{2}\right)=2 Q(S)-1$ ), so we can apply $7.6(b)$ to obtain

$$
D^{w}\left(4 S^{2}\right)-2 Q(S) D^{w}(x)=0 .
$$

Subtracting this from four times the previous equation, one obtains the result. q.e.d.

A trivial extension of this argument yields

Lemma 7.11. Let $X$ be a K3 with its standard homology-orientation, and let $w$ be a line bundle. Then for all $r \geq 0$ there is a with $\left(w^{\prime}\right)^{2}=w^{2}+2 r \bmod 4$ such that

$$
D^{w}\left(x^{r}\right)=(-2)^{r} D^{w^{\prime}}(1) .
$$

Proof. Use induction on $r$. For the induction step, follow the proof of the previous Lemma, but apply Proposition 7.6(c) and (b) with $z=x^{r-1}$ instead of $z=1$. $\quad$ q.e.d.

The next lemma, when combined with Lemma 7.9, gives the entire invariant.

Lemma 7.12. Let $X$ be a $K 3$ with its standard homology-orientation, and let $w$ be a line bundle. Then given $r$ and $i \geq 0$ there is a $w^{\prime}$ with $\left(w^{\prime}\right)^{2}=w^{2}+2(r+i) \bmod 4$ such that

$$
D^{w}\left(x^{r} h^{2 i}\right)=2^{r}(-1)^{r+i} \frac{(2 i) !}{2^{i} i !} Q(h)^{i} D^{w^{\prime}}(1),
$$

for all $h \in H_{2}(X)$.

Proof. The case $i=0$ is the previous Lemma. We proceed by induction on $i$. We can restrict our attention to classes $h$ in the integer lattice. Given such an $h$, let $S$ be an integral class orthogonal to both $h$ and $w$, with the property that $Q(S)$ is large and equal to $0 \bmod 4$, so that $S$ can be represented by a tight surface $\Sigma$ of odd genus. It is well known [33] that the action of the diffeomorphism group of $X$ on 
its homology is large enough to ensure that, with $w, r$ and $i$ fixed, the function

$$
D^{w}\left(x^{r} h^{2 i}\right)
$$

is a multiple of $Q(h)^{i}$. Using the polarization identity we can therefore deduce:

$$
D^{w}\left(x^{r} h^{2 i}\right)=\frac{Q(h)}{Q(S)}(2 i-1) D^{w}\left(x^{r} S^{2} h^{2 i-2}\right) .
$$

Now apply the universal relation of Proposition 7.6(b) with $z=x^{r} h^{2 i-2}$ to obtain

$$
\begin{aligned}
D^{w}\left(x^{r} S^{2} h^{2 i-2}\right) & =(g-1) D^{w}\left(x^{r+1} h^{2 i-2}\right) \\
& =\frac{1}{2} Q(S) D^{w}\left(x^{r+1} h^{2 i-2}\right),
\end{aligned}
$$

and hence

$$
D^{w}\left(x^{r} h^{2 i}\right)=\frac{1}{2}(2 i-1) Q(h) D^{w}\left(x^{r+1} h^{2 i-2}\right) .
$$

This reduces the calculation to a case with smaller $i$ and larger $r$, and the result follows. q.e.d.

We summarize the result of Lemmas 7.9 and 7.12:

Proposition 7.13. The K3 surface has simple type. For all $w$, the Donaldson series $\mathscr{D}^{w}$ is given by

$$
\mathscr{D}^{w}=(-1)^{w^{2} / 2} \exp \left(\frac{Q}{2}\right)
$$

with the standard homology-orientation.

Proof. From Lemmas 7.9 and 7.12 we obtain

$$
D^{w}\left(x^{r} h^{2 i}\right)= \begin{cases}-2^{r}(-1)^{r+i} \frac{(2 i) !}{2^{i} i !} Q(h)^{i}, & w^{2}+2(r+i)=2 \bmod 4 \\ 0, & \text { otherwise }\end{cases}
$$

which we can rephrase as

$$
D^{w}\left(x^{r} h^{2 i}\right)= \begin{cases}2^{r}(-1)^{w^{2} / 2} \frac{(2 i) !}{2^{i} i !} Q(h)^{i}, & w^{2}+2(r+i)=2 \bmod 4 \\ 0, & \text { otherwise }\end{cases}
$$

From this we obtain

$$
D^{w}\left(\left(1+\frac{1}{2} x\right) h^{2 i}\right)=(-1)^{w^{2} / 2} \frac{(2 i) !}{2^{i} i !} Q(h)^{i},
$$


and hence

$$
\begin{aligned}
\mathscr{D}^{w}(h) & =D^{w}\left(\left(1+\frac{1}{2} x\right) e^{h}\right) \\
& =\sum_{i} D^{w}\left(\left(1+\frac{1}{2} x\right) h^{2 i}\right) /(2 i) ! \\
& =(-1)^{w^{2} / 2} \sum_{i} \frac{Q(h)^{i}}{2^{i} i !} Q(h)^{i} \\
& =(-1)^{w^{2} / 2} \exp \left(\frac{Q}{2}\right) \cdot \quad \text { q.e.d. }
\end{aligned}
$$

(iii) Simple type and universal relations. The universal relations described in Theorem 7.4 take a simpler form when $X$ has simple type and $b^{1}(X)=0$. Recall from section 1 that when $X$ has simple type, we define

$$
{ }^{+} D^{w}(z)=D^{w}\left(\left(1+\frac{1}{2} x\right) z\right),
$$

to obtain a linear function ${ }^{+} D^{w}: \mathbb{A}(X) \rightarrow \mathbb{R}$ satisfying

$$
{ }^{+} D^{w}(x z)=2{ }^{+} D^{w}(z)
$$

for all $z$. When $\Sigma$ is an oriented embedded surface, we define

$$
{ }^{+} D^{w, p}(z)=D^{w, p}\left(\left(1+\frac{1}{2} x\right) z\right)
$$

in a similar way, whenever $p$ satisfies the parity condition 2.20 . Theorem 5.10, which expresses the invariants of the pair in terms of the ordinary invariants, for $p$ in a certain range, takes the following form in the case of simple type:

Proposition 7.16. Let $X$ be a manifold of simple type with $b^{1}(X)=0$. Let $\imath: \Sigma \hookrightarrow X$ be the inclusion of an oriented, embedded surface of genus at least 2 , representing a class $S \in H_{2}(X ; \mathbb{Z})$ with $Q(S)=n>0$, and let $\mathrm{f}$ denote the inclusion $\mathrm{J}: X / \Sigma \rightarrow X$. Let $p$ and $w$ satisfy the parity condition $(2.20)$, so that ${ }^{+} D^{w, p}$ is defined, and suppose that

$$
p \leq \frac{1}{2} \Delta(n, g) \text {. }
$$

Then there is a polynomial $\eta_{p}^{w}(t) \in \mathbb{Q}[t]$, inhomogeneous of degree $p$, such that

$$
{ }^{+} D^{w, p}(z)=2^{g+} D^{w}\left(\imath_{*}\left(\eta_{p}^{w}(S)\right) \jmath_{*}(z)\right)
$$


for all $z \in \mathbb{A}(X / \Sigma)$. The polynomial $\eta_{p}^{w}(t)$ depends only on $Q(\Sigma)$, genus $(\Sigma)$ and $w \cdot \Sigma$ mod 2 . It does not depend on $X$ or $z$. The coefficient of $t^{p}$ in $\eta_{p}^{w}$ is $1 / p$ !. The polynomial is either odd or even, according to the parity of $p$.

Proof. In the algebra $\mathbb{A}(\Sigma)$, let $I$ be the ideal generated by $(2-x)$ and $H_{1}(\Sigma)$. Because $X$ has simple type and no first homology, we have

$$
{ }^{+} D^{w}\left(\imath_{*}(w) \jmath_{*}(z)\right)=0
$$

for all $w \in I$. Let $\omega_{p}^{w}$ be the elements of $\mathbb{A}(\Sigma)$ from Theorem 5.10. There is a unique element $\eta_{p}^{w}(S) \in \operatorname{Sym}\left(H_{2}(\Sigma ; \mathbb{R})\right)$ equal to $\omega_{p}^{w}$ modulo $I$ : it is obtained by writing out $\omega_{p}^{w}$ in terms of the standard generators, deleting any term involving $H_{1}(\Sigma)$, and replacing $x$ by 2 . The Proposition then follows. The value of the leading coefficient comes from (6.3). The final statement follows from the fact that $\omega_{p}^{w}$ has terms only in degrees equal to $2 p \bmod 8$, because the only inhomogeneous generator of $I$ is $2-x$, which involves only degrees divisible by 4 . (Note that $S$ has degree 2, so a polynomial of degree $p$ in $S$ is an element of $\mathbb{A}(X)$ of degree $2 p$.) q.e.d.

From this, one obtains the following result: a version of Theorem 7.4 for the case of simple type. In this statement, we again omit $\imath_{*}$ and $\jmath_{*}$ from our notation.

Proposition 7.17. Let $X$ be a manifold of simple type with $b^{1}(X)=0$. Let $\imath: \Sigma \hookrightarrow X$ be an embedded, oriented surface of genus at least 2 , representing a class $S \in H_{2}(X ; \mathbb{Z})$ with $Q(S)=n>0$. Let $p$ satisfy the parity condition $(2.20)$, let $p^{\prime}=a(\Sigma)-p$, and suppose that

$$
\max \left\{p, p^{\prime}\right\} \leq \frac{1}{2} \Delta(n, g) .
$$

Then there is a relation

$$
{ }^{+} D^{w}\left(\eta_{p}^{w}(S) z\right)=(-1)^{p^{+}+} D^{w^{\prime}}\left(\eta_{p^{\prime}}^{w^{\prime}}(S) z\right),
$$

valid for all $z \in \mathbb{A}(X / \Sigma)$. Here $\eta_{p}^{w}$ is the polynomial of degree $p$ which arises in the previous Proposition. If either $p$ or $p^{\prime}$ is negative, the corresponding side of the equation is to be interpreted as zero.

The relations described by this Proposition are most often useful when $p>a(\Sigma)$, so that the right-hand side is zero. In this case, they do not involve $w^{\prime}$. Let us spell out the nature of these relations and 
their consequences. Let $(X, \Sigma)$ be as in the Proposition, let $w$ be a line bundle, let $z \in \mathbb{A}(X / \Sigma)$, and consider

$$
{ }^{+} D^{w}\left(S^{d} z\right)
$$

as a function of $d$. The above Proposition gives linear relations among the values of ${ }^{+} D^{w}\left(S^{d} z\right)$, for different $d$ satisfying

$$
d \equiv(g-1)-w \cdot S(\bmod 2) .
$$

The relations have the following consequence.

Proposition 7.19. Suppose that $Q(S)=n$ is at least 20 and that

$$
a(\Sigma) \leq \frac{1}{6}(n-20) .
$$

Let $a^{+}$be the smallest integer which is strictly greater than $a(\Sigma)$ and has the same parity as $(g-1)-w \cdot \Sigma$. Then the values of ${ }^{+} D^{w}\left(S^{d} z\right)$, for $d$ satisfying 7.18 , satisfy universal linear relations: the relations determine ${ }^{+} D^{w}\left(S^{d} z\right)$ for all $d \geq a^{+}$in terms of values of ${ }^{+} D^{w}\left(S^{d} z\right)$ for $d<a^{+}$. The relations depend only on $g, n$ and the value of $w \cdot \Sigma \bmod$ 2; they are otherwise independent of $(X, \Sigma)$ and independent of $z$.

Remark. We have not taken pains to give the sharpest possible bounds on $a(\Sigma)$ and $n$ here.

Proof of Proposition 7.19. For all $p \equiv(g-1)-w \cdot S \bmod 2$ in the range

$$
a^{+} \leq p \leq \frac{1}{2} \Delta(n, g),
$$

Proposition 7.17 gives a relation

$$
{ }^{+} D^{w}\left(\eta_{p}^{w}(S) z\right)=0,
$$

which, when written out long-hand, takes the form

$$
(1 / p !)^{+} D^{w}\left(S^{p} z\right)+\kappa_{p-2}^{+} D^{w}\left(S^{p-2} z\right)+\cdots=0 .
$$

This relation determines ${ }^{+} D^{w}\left(S^{p} z\right)$ in terms of ${ }^{+} D^{w}\left(S^{d} z\right)$ for $d<p$, of the same parity. This would be the end of the proof if it were not for the upper bound on $p$ in 7.21. To circumvent the upper bound on $p$ and so obtain relations which determine ${ }^{+} D^{w}\left(S^{d} z\right)$ for all larger $d$, we exploit the following device. Let $\Sigma_{m}$ be the embedded surface 
representing $m S$, as in Lemma 7.7. Let $\tilde{\Sigma}_{m}$ be either $\Sigma_{m}$ or $\Sigma_{m} \# T^{2}$ (the internal connected sum of $\Sigma_{m}$ and a null-homologous torus); we choose one or the other to ensure that

$$
\left(\operatorname{genus}\left(\tilde{\Sigma}_{m}\right)-1\right)-w \cdot \tilde{\Sigma}_{m} \equiv(\operatorname{genus}(\Sigma)-1)-w \cdot \Sigma \quad(\bmod 2) .
$$

Let $\tilde{g}, \tilde{n}, \tilde{a}^{+}$etc. be the corresponding quantities for $\tilde{\Sigma}_{m}$; so for example, $\tilde{n}=m^{2} n$.

The inequality (7.20) is designed to ensure that the upper bound on the right-hand side of (7.21) is not less than $2 a+4$, so our original $\Sigma$ gives us valid relations for $p$ in the range

$$
a^{+} \leq p \leq 2 a+4 \text {. }
$$

Because $\tilde{n}$ grows quadratically with $m$ while $\tilde{a}$ only grows linearly, the surfaces $\tilde{\Sigma}_{m}$ also satisfy (7.20), so we can use them also to obtain relations on ${ }^{+} D^{w}\left((m S)^{p} z\right)$ (or equivalently ${ }^{+} D^{w}\left(S^{p} z\right)$ ) for $p$ in the range

$$
\tilde{a}^{+} \leq p \leq 2 \tilde{a}+4
$$

These ranges overlap. Indeed, when $m=2, \tilde{a}^{+}$is not greater than $2 a+4$ : this is because, $\tilde{a}$ is either $2 a$ or $2 a+2$, and $\tilde{a}^{+}$is at most $\tilde{a}+2$. So taking $m$ to be each power of 2 in turn (for example), we obtain relations which determine all ${ }^{+} D^{w}\left(S^{d} z\right)$, for $d$ of the correct parity, in terms of the values for $d<a^{+}$. q.e.d.

We can recast this result in terms of the analytic function or formal power series

$$
\mathscr{D}^{w}(h)={ }^{+} D^{w}\left(e^{h}\right) .
$$

Let $S$ be an integral homology class as usual, and consider the formal power series

$$
\mathscr{D}^{w}(t S)=\sum t^{d+} D^{w}\left(S^{d}\right) / d ! \in \mathbb{R}[[t]] .
$$

This power series is either even or odd, depending on the parity of $w^{2}+\left(b^{+}+1\right) / 2$. Let us describe an embedded surface $\Sigma$ representing $S$ as fitting if $(g-1)-w \cdot S$ has the same parity as $w^{2}+\left(b^{+}+1\right) / 2$. In this case, the above Proposition gives us linear relations on the coefficients of the power series. The following Corollary can be deduced directly.

Corollary 7.22. Let $\left(X_{1}, \Sigma_{1}\right)$ and $\left(X_{2}, \Sigma_{2}\right)$ be two pairs, and let $w_{i} \rightarrow X_{i}$ be line bundles. Suppose each $X_{i}$ has simple type with $b^{1}=$ 
0 , suppose that $\Sigma_{1}$ and $\Sigma_{2}$ have the same genus and self-intersection number and that $w_{1} \cdot \Sigma_{1}=w_{2} \cdot \Sigma_{2}$ mod 2. Suppose also that both $\Sigma_{1}$ and $\Sigma_{2}$ are fitting, in the sense above, and the inequality 7.20 is satisfied. Then we have

$$
\mathscr{D}_{X_{1}}^{w_{1}}\left(t S_{1}\right)=\mathscr{D}_{X_{2}}^{w_{2}}\left(t S_{2}\right)
$$

provided only that these two power series agree up to and including the term in $t^{a}$, where $a=a\left(\Sigma_{1}\right)=a\left(\Sigma_{2}\right)$.

More generally, if we have $r$ such pairs $\left(X_{i}, \Sigma_{i}\right)(i=1, \ldots, r)$, and if there are constants $\alpha_{i}$ such that

$$
\alpha_{1} \mathscr{D}_{X_{1}}^{w_{1}}\left(t S_{1}\right)+\cdots+\alpha_{r} \mathscr{D}_{X_{r}}^{w_{r}}\left(t S_{r}\right)=o\left(t^{a}\right)
$$

then the left-hand side is identically zero. (The notation o $\left(t^{a}\right)$ indicates a general element of $t^{a+1} \mathbb{R}[[t]]$.)

(iv) $K 3$ blown up. Let $X$ be $K 3$ with its standard homology orientation, and let $\tilde{X}$ be $X \# p\left(\overline{\mathbb{C P}}^{2}\right)$ (or $K 3$ 'blown up at $p$ points'), with the homology orientation that it acquires as a result of the fact that $H^{2}(X)$ is naturally a summand of $H^{2}(\tilde{X})$ and that a maximal positive subspace in the one is also maximal in the other. Let $w$ be a line bundle on $\tilde{X}$ which is trivial on standard spheres $C_{1}, \ldots, C_{p}$ of degree 1 in the $p$ copies of $\overline{C P}^{2}$, so $w$ can be regarded as coming from $X$ under the 'blow-down' map which collapses these spheres. Let $E_{1}$, $\ldots, E_{p}$ be the cohomology classes represented by $C_{1}, \ldots, C_{p}$.

Proposition 7.23. The manifold $\tilde{X}=K 3 \# p\left(\overline{\mathbb{C P}}^{2}\right)$ has simple type. For any $w$ which is trivial on the $E_{i}$, the Donaldson series is given by

$$
\mathscr{D}^{w}=(-1)^{w^{2} / 2} \exp \left(\frac{Q_{\tilde{X}}}{2}\right) \prod_{i=1}^{p} \cosh E_{i} .
$$

Here the homology classes $E_{i}$ are regarded as defining cohomology classes, through Poincaré duality. More generally, for the line-bundle $\bar{w}=$ $w+E_{1}+\cdots+E_{q}(q \leq p)$, one has

$$
\mathscr{D}^{\bar{w}}=(-1)^{q+w^{2} / 2} \exp \left(\frac{Q_{\tilde{X}}}{2}\right) \prod_{i=1}^{q} \sinh E_{i} \prod_{i=q+1}^{p} \cosh E_{i} .
$$

Proof. We proceed through a number of lemmas. The first four are well known (if not quite in this form). The fourth can be proved using 
Floer homology and a splitting of $K 3$, but we have preferred to avoid those techniques: we base our arguments on the universal relations. We start with the case $p=1$.

Lemma 7.24. The manifold $\tilde{X}=X \# \overline{\mathbb{C P}}^{2}$ has simple type. There are power series $J$ and $L$ such that the invariants of $\tilde{X}$ are given by

$$
\begin{aligned}
\mathscr{D}^{w} & =(-1)^{w^{2} / 2} \exp \left(\frac{Q_{\tilde{X}}}{2}\right) J(E), \\
\mathscr{D}^{w+E} & =(-1)^{w^{2} / 2} \exp \left(\frac{Q_{\tilde{X}}}{2}\right) L(E) .
\end{aligned}
$$

The series $J$ is even, and $L$ is odd.

Proof. Let $\bar{w}$ be either $w$ or $w+E$, and consider the invariants $D_{\tilde{X}}^{\bar{w}}$. The proof of Lemma 7.12 was based entirely on the geometry of certain embedded surfaces in $K 3$ and the diffeomorphism group of the manifold. The argument adapts without change to prove that, for any $d$, and any $h \in H_{2}(X) \subset H_{2}(\tilde{X})$,

$$
D_{\bar{X}}^{\bar{w}}\left(x^{r} h^{2 i} E^{d}\right)=2^{r}(-1)^{r+i} \frac{(2 i) !}{2^{i} i !} Q(h)^{i} D_{\tilde{X}}^{\bar{w}^{\prime}}\left(E^{d}\right),
$$

where, as in (7.12), the first Chern class of $\bar{w}^{\prime}$ satisfies $\left(\bar{w}^{\prime}\right)^{2}=\bar{w}^{2}+$ $2(r+i) \bmod 4$. Furthermore, $\bar{w}^{\prime}$ can be taken to have the form $w^{\prime}$ or $w^{\prime}+E$, for some $w^{\prime}$ coming from $X$, according as $\bar{w}$ is $w$ or $w+E$ respectively.

It follows that $\tilde{X}$ has simple type, so we may define the simple-type invariants ${ }^{+} D^{\bar{w}}$. It is a consequence of the action of diffeomorphisms and the formulae above that ${ }^{+} D^{\bar{w}}$ depends on $w$ only through the value of $w^{2} / 2 \bmod 2$, and then only in its sign. So the relation

$$
{ }^{+} D_{\bar{X}}^{\bar{w}}\left(h^{2 i} E^{d}\right)=(-1)^{i} \frac{(2 i) !}{2^{i} i !} Q(h)^{i+} D_{\bar{X}}^{\bar{w}^{\prime}}\left(E^{d}\right),
$$

for $h \in H_{2}(X)$, which is derived from the one above, can be rewritten

$$
{ }^{+} D_{\bar{X}}^{\overline{\tilde{W}}}\left(h^{2 i} E^{d}\right)=\frac{(2 i) !}{2^{i} i !} Q(h)^{i+} D_{\bar{X}}^{\bar{w}}\left(E^{d}\right) .
$$

Now set

$$
\begin{aligned}
& a_{d}=(-1)^{w^{2} / 2+} D^{w}\left(E^{d}\right) \\
& b_{d}=(-1)^{w^{2} / 2+} D^{w+E}\left(E^{d}\right) .
\end{aligned}
$$


These do not depend on $w$. Define power series $J$ and $L$ by

$$
\begin{aligned}
& J(t)=e^{t^{2} / 2} \sum_{d}(-1)^{d} a_{d} t^{d} / d ! \\
& L(t)=e^{t^{2} / 2} \sum_{d}^{d}(-1)^{d} b_{d} t^{d} / d !
\end{aligned}
$$

Because the invariants ${ }^{+} D^{w}$ and ${ }^{+} D^{w+E}$ are even and odd respectively as functions on $H_{2}(\hat{X})$, so are the power series $J$ and $L$. The most general second homology class in $\tilde{X}$ can be written $T=h+\lambda E$, for some $h \in H_{2}(X)$, and with the binomial theorem we calculate

$$
\begin{aligned}
\mathscr{D}^{w}(T) & =\mathscr{D}^{w}(h+\lambda E) \\
& =\sum_{d}{ }^{+} D^{w}\left((h+\lambda E)^{d}\right) / d ! \\
& =\sum_{d} \sum_{i}\left(\begin{array}{c}
d \\
2 i
\end{array}\right) \lambda^{d-2 i+} D^{w}\left(h^{2 i} E^{d-2 i}\right) / d ! \\
& =\sum_{d} \sum_{i}\left(\frac{1}{(2 i) !(d-2 i) !}\right)\left(\frac{(2 i) !}{2^{i} i !}\right) Q(h)^{i} \lambda^{d-2 i+} D^{w}\left(E^{d-2 i}\right) \\
& =\exp \left(\frac{Q(h)}{2}\right) \sum_{d} \lambda^{d}{ }^{+} D^{w}\left(E^{d}\right) / d ! \\
& =(-1)^{w^{2} / 2} \exp \left(\frac{Q_{\tilde{X}}(T)}{2}\right) e^{\lambda^{2} / 2} \sum_{d} a_{d} \lambda^{d} / d ! \\
& =(-1)^{w^{2} / 2} \exp \left(\frac{Q_{\tilde{X}}(T)}{2}\right) e^{(E \cdot T)^{2} / 2} \sum_{d}(-1)^{d} a_{d}(E \cdot T)^{d} / d ! \\
& =(-1)^{w^{2} / 2} \exp \left(\frac{Q_{\tilde{X}}(T)}{2}\right) J(E \cdot T) .
\end{aligned}
$$

(In the sixth and seventh lines we use the fact that $E^{2}=-1$.) This verifies the shape of the formula for $\mathscr{D}^{w}$, and the calculation for $\mathscr{D}^{w+E}$ is identical. q.e.d.

Lemma 7.25. The constant term in $J(t)$ is 1 .

Proof. This arises from the statement (4.8) in [10], that the restriction of ${ }^{+} D_{\tilde{X}}^{w}$ to $H_{2}(X)$ is equal to ${ }^{+} D_{X}^{w}$. $\quad$ q.e.d.

Lemma 7.26. The coefficient of $t$ in $L(t)$ is -1 .

Proof. This is just a statement of the result of Kotschick [23], adapted to the present notation. We give an alternative derivation, based on the universal relations. 
Let $S$ be an integral homology class in $X$ with $Q(S)$ large and positive. Put

$$
\tilde{S}=S+E \in H_{2}(\tilde{X}) .
$$

Represent $S$ by a tight surface $\Sigma$, and let $\tilde{\Sigma}$ be a surface representing $\tilde{S}$, formed as

$$
\tilde{\Sigma}=\Sigma \# C .
$$

For convenience, make $S$ divisible by 2 in the integer lattice, so that ${ }^{+} D^{S}={ }^{+} D^{0}$. We apply the relations of Proposition 7.17 for the pair $(\tilde{X}, \tilde{\Sigma})$ with $p=1$ and $w=E$. Because $a(\tilde{\Sigma})=1$ we have $p^{\prime}=0$. From (6.2.) we have $\eta_{1}^{w}(t)=t$ and $\eta_{0}^{w}=1$, so the relation says

$$
{ }^{+} D_{\tilde{X}}^{E}(\tilde{\Sigma})={ }^{+} D_{\tilde{X}}^{0}(1) .
$$

Using the expressions in the previous lemma, we see that the left-hand side is minus the coefficient of $t$ in $L(t)$. The right-hand side is $J(0)$. So the result follows from the previous lemma. q.e.d.

We now return to the case of general $p>1$. Let $\tilde{X}=X \# p\left(\overline{\mathbb{C P}}^{2}\right)$ again, and $\bar{w}=w+E_{1}+\cdots+E_{q}$, as in Proposition 7.23. The same argument as above shows that $\tilde{X}$ has simple type. Furthermore, we have

Lemma 7.27. The Donaldson series of $\tilde{X}$ for the line-bundle $\bar{w}$ has the form

$$
\mathscr{D}^{\bar{w}}=(-1)^{w^{2} / 2} \exp \left(\frac{Q_{\bar{X}}}{2}\right) \prod_{i=1}^{q} L\left(E_{i}\right) \prod_{i=q+1}^{p} J\left(E_{i}\right),
$$

where $J$ and $L$ are the power series from Lemma 7.24.

Proof. Write $\mathbf{E}=\left(E_{1}, \ldots, E_{p}\right)$, and for any multi-index $\mathbf{d}$ write

$$
\mathbf{E}^{\mathbf{d}}=\prod E_{i}^{d_{i}} .
$$

The proof of Lemma 7.24 extends to show that there is a power series $\mathbf{L}$ in $p$ variables such that

$$
\mathscr{D}_{\bar{X}}^{\bar{w}}=(-1)^{w^{2} / 2} \exp \left(\frac{Q_{\tilde{X}}}{2}\right) \mathbf{L}(\mathbf{E}),
$$

where

$$
\mathbf{L}(\mathbf{t})=\exp \left(\frac{\sum t_{i}^{2}}{2}\right) \sum_{\mathbf{d}}(-1)^{d_{1}+\cdots+d_{p}} b_{\mathbf{d}} \frac{t_{1}^{d_{1}} \cdots t_{p}^{d_{p}}}{d_{1} ! \cdots d_{p} !}
$$


and

$$
b_{\mathbf{d}}=(-1)^{w^{2} / 2+} D_{\tilde{X}}^{\bar{w}}\left(\mathbf{E}^{\mathbf{d}}\right) .
$$

Also, $\mathbf{L}$ is odd in the first $q$ variables and even in the remaining variables, and the proof of (7.26) shows that the coefficient of $t_{1} t_{2} \cdots t_{q}$ is 1 .

To show that $\mathbf{L}$ is a product as stated, what we need to establish is the following. Fix $j$ with $1 \leq j \leq p$, and write

$$
\begin{aligned}
\hat{\mathbf{t}} & =\left(t_{1}, \ldots, \hat{t}_{j}, \ldots t_{p}\right), \\
\hat{\mathbf{E}} & =\left(E_{1}, \ldots, \hat{E}_{j}, \ldots E_{p}\right) .
\end{aligned}
$$

Fix a $(p-1)$-multi-index $\hat{\mathbf{d}}$ and let $\hat{L}\left(t_{j}\right)$ be the coefficient of $\hat{\mathbf{t}}^{\hat{\mathbf{d}}}$ in $\mathbf{L}(\mathbf{t})$, so $\hat{L}$ is a power series of one variable. We need to show that $\hat{L}$ is a multiple of $L$,

$$
\hat{L}(t)=\alpha L(t)
$$

if $j \leq q$, and a multiple of $J(t)$ otherwise. The constant $\alpha$, of course, will depend on $\hat{\mathbf{d}}$.

Let $\Sigma$ again be a tight surface in $X$ representing a class $S$ with $Q(S)$ large and positive. Arrange that the genus of $\Sigma$ is odd and $w \cdot S$ is zero, so that $\Sigma$ is fitting. Let $\tilde{\Sigma} \subset \tilde{X}$ be a surface representing $\tilde{S}=S+E_{j}$, formed by summing $\Sigma$ with a sphere:

$$
\tilde{\Sigma}=\Sigma \# C_{j} .
$$

Let $\tilde{X}_{1}$ be $X$ blown up at one point (rather than $p$ ), and let $\tilde{\Sigma}_{1}$ be a similar surface representing $\tilde{S}_{1}=S+E$ in $\tilde{X}_{1}$. Let $w_{1} \rightarrow X_{1}$ be $w+E$ if $j \leq q$ and $w$ otherwise. The surface $\tilde{\Sigma}_{1}$ is fitting in $\tilde{X}_{1}$ for the line bundle $w_{1}$. Consider the two power series

$$
\begin{aligned}
\Lambda(t) & =\mathscr{D}_{\tilde{X}_{1}}^{w_{1}}\left(t \tilde{S}_{1}\right) \\
& =\sum_{d} t^{d+} D_{\tilde{X}_{1}}^{w_{1}}\left(\tilde{S}_{1}^{d}\right) / d !
\end{aligned}
$$

and

$$
\hat{\Lambda}(t)=\sum_{d} t^{d+} D_{\tilde{X}}^{\bar{w}}\left(\tilde{S}^{d} \hat{\mathbf{E}}^{\hat{\mathbf{d}}}\right) / d !
$$

These two power series are either odd or even together, depending on $j$. Because $\tilde{S}$ and $\tilde{S}_{1}$ have the same self-intersection number and both have 
$a=1$, we can apply Proposition 7.19 to see that there are universal linear relations (the same for $\Lambda$ as $\hat{\Lambda}$ ) which determine the coefficient of $t^{d}$ for all $d>1$ in terms of the leading coefficient (that of $t^{1}$ or $t^{0}$ respectively). It follows that $\hat{\Lambda}$ is a multiple of $\Lambda$ (since the latter is non-zero), and this is entirely equivalent to (7.28). q.e.d.

Lemma 7.29. The power series $J$ and $L$ satisfy

$$
\begin{aligned}
& J(2 t)=2 J(t)^{2}-1, \\
& L(2 t)=2 J(t) L(t) .
\end{aligned}
$$

Proof. Let $\tilde{X}$ be $X$ blown up at two points. Let $w$ be the trivial line bundle on $\tilde{X}$. Fix a large positive $n$ equal to $2 \bmod 4$, and consider three surfaces $\Sigma_{1}, \Sigma_{2}$ and $\Sigma_{3}$ with $Q\left(\Sigma_{i}\right)=n$ and $a\left(\Sigma_{i}\right)=2$. (The condition $n \equiv 2 \bmod 4$ means that the genus of these surfaces is odd, so they are fitting.) The surfaces are to be constructed as follows.

For $\Sigma_{1}$, start with a tight surface $\Sigma^{\prime}$ in $K 3$ with $Q\left(\Sigma^{\prime}\right)=n+2$, and let

$$
\Sigma_{1}=\Sigma^{\prime} \# \bar{C}_{1} \# \bar{C}_{2}
$$

For $\Sigma_{2}$, start with a tight surface $\Sigma^{\prime \prime}$ in $K 3$ with $Q\left(\Sigma^{\prime \prime}\right)=n$, and let

$$
\Sigma_{2}=\Sigma^{\prime \prime} \# T^{2}
$$

where $T^{2}$ is a null-homologous torus. For $\Sigma_{3}$, let $S$ be a class in $H_{2}(X)$ with $Q(S)=n+4$, and seek a surface with homology class

$$
\left[\Sigma_{3}\right]=S-2 E_{1}
$$

To find such a surface with $a\left(\Sigma_{3}\right)=2$, start with an immersed surface $\Sigma$ in $X$ representing $S$, with genus satisfying $2 g-2=n+2$ and having a single transverse self-intersection point of positive sign. An example of such a surface with the correct genus would be an algebraic curve with a single ordinary double-point. It is, however, quite easy to construct such a surface, for any desired $n$, by imitating the proof of Lemma 7.8. Now blow up $X$ at two points, the first being the double-point of $\Sigma$ and the second being away from $\Sigma$. Let $\Sigma_{3}$ be the proper transform of $\Sigma$ (in the topological sense of [18]), which is a surface of the same genus, representing the class $S-2 E_{1}$ as desired. Let $S_{1}, S_{2}, S_{3}$ be the homology classes represented by the three surfaces. 
From Lemma 7.27 we have

$$
\begin{aligned}
& \mathscr{D}^{w}\left(t S_{1}\right)=e^{n t^{2} / 2} J(t)^{2} \\
& \mathscr{D}^{w}\left(t S_{2}\right)=e^{n t^{2} / 2} \\
& \mathscr{D}^{w}\left(t S_{3}\right)=e^{n t^{2} / 2} J(2 t) .
\end{aligned}
$$

Because $J(t)$ is an even series with constant term 1 , we have

$$
J(2 t)=2 J(t)^{2}-1+O\left(t^{4}\right)
$$

so

$$
\mathscr{D}^{w}\left(t S_{3}\right)=2 \mathscr{D}^{w}\left(t S_{1}\right)-\mathscr{D}^{w}\left(t S_{2}\right)+O\left(t^{4}\right) .
$$

Since $a=2$, we can apply the universal relations, in the shape of Corollary 7.22, to conclude that the terms in $O\left(t^{4}\right)$ vanish, and hence

$$
J(2 t)=2 J(t)^{2}-1
$$

For the second of the two formulae, take $\tilde{X}$ as before, and consider the two surfaces $\Sigma_{1}$ and $\Sigma_{3}$ above. Let $w$ be a line bundle on $X$ with $w \cdot \Sigma_{3}=1$ and $w^{2}=0 \bmod 4$ (such a $w$ can be found as long as the homology class of $\Sigma$ above is not divisible by 2.) Set $w_{1}=E_{1}$ and $w_{3}=w+E_{1}$. Then $w_{1} \cdot S_{1} \equiv w_{3} \cdot S_{3} \equiv 1 \bmod 2$, and both surfaces are fitting. We have

$$
\begin{aligned}
& \mathscr{D}^{w_{1}}\left(t S_{1}\right)=e^{n t^{2} / 2} L(t) J(t), \\
& \mathscr{D}^{w_{3}}\left(t S_{3}\right)=e^{n t^{2} / 2} L(2 t) .
\end{aligned}
$$

Since $L(t)=-t+O\left(t^{3}\right)$ and $J(t)=1+O\left(t^{2}\right)$, we have

$$
\mathscr{D}^{w_{3}}\left(t S_{3}\right)=2 \mathscr{D}^{w_{1}}\left(t S_{1}\right)+O\left(t^{3}\right) \text {. }
$$

Again, Corollary 7.22 shows that the equality is exact to all orders, so

$$
L(2 t)=2 J(t) L(t) . \quad \text { q.e.d. }
$$

The proof of Proposition 7.23 is now complete: it is an elementary exercise to show that the only pair of power series $J(t)$ and $L(t)$ satisfying the relations of (7.29) and the initial conditions of (7.25) and (7.26) are

$$
\begin{aligned}
& J(t)=\cosh (t), \\
& L(t)=-\sinh (t),
\end{aligned}
$$


so the final result follows from Lemma 7.27. q.e.d.

(v) The structure of the invariants on a ray. Having found the invariants of $K 3$ blown up, we are now ready to treat a more general manifold. Let $X$ be a manifold of simple type with $b^{1}=0$, and let $\Sigma$ be an embedded surface representing a class $S$ with $Q(S)>0$. Pick any $w \rightarrow X$, and consider the restriction of $\mathscr{D}^{w}$ to the ray $\{t S\}$. Because $\mathscr{D}^{w}$ is a priori only a formal power series, the restriction is formal too: we simply mean the power series $\mathscr{D}^{w}(t S)$ in $t$.

Proposition 7.30. Let $(X, \Sigma)$ and $w$ be as above, so $\Sigma$ represents $S$ with $Q(S)>0$, and let $a=a(\Sigma)$. Then there exist constants $\beta_{r}$ such that

$$
\mathscr{D}_{X}^{w}(t S)= \begin{cases}\exp \left(\frac{Q(t S)}{2}\right) \sum_{r} \beta_{r} \cosh (r t), & \text { or } \\ \exp \left(\frac{Q(t S)}{2}\right) \sum_{r} \beta_{r} \sinh (r t), & \end{cases}
$$

according as the invariant $\mathscr{D}_{X}^{w}$ is even or odd. The sum extends over integers $r$ in the range $0 \leq r \leq a$ satisfying $r \equiv Q(S) \bmod 2$.

Proof. Write $n=Q(S)$. At the outset, we make two additional assumptions on $\Sigma$. The first is that $\Sigma$ is fitting: i.e. that $(g-1)-w \cdot \Sigma$ is even if $\mathscr{D}^{w}$ is even, or odd if $\mathscr{D}^{w}$ is odd. The second is that $n$ is large compared to $a$, in the sense required by Propositions 7.19 and 7.22: that is, the inequality (7.20) should hold.

Let $\tilde{X}$ denote $K 3$ blown up at $N$ points, where $N$ is any number larger than $a$. (We continue to use $X$ to denote the general 4-manifold of the Proposition, and hope this does not cause confusion.) For $0 \leq$ $r \leq a$, with $r \equiv a \bmod 2$, construct a surface $\Sigma_{r} \subset \tilde{X}$ as follows. Start with a tight surface $\Sigma^{\prime} \subset K 3$ representing a class $S^{\prime}$ of square $n+r$. (Note that $n \equiv r \equiv a \bmod 2$, so $n+r$ is even). Let

$$
\Sigma_{r}=\Sigma^{\prime} \#\left(\bar{C}_{1} \# \cdots \# \bar{C}_{r}\right) \#\left(\frac{1}{2}(a-r) T^{2}\right) .
$$

This surface represents the class

$$
S_{r}=S^{\prime}-E_{1}-\cdots-E_{r} .
$$

It has the same invariants as $\Sigma$, in that $Q\left(\Sigma_{r}\right)=n$ and $a\left(\Sigma_{r}\right)=a$. We choose $S^{\prime}$ so that it is not divisible by 2 . There is a $w_{1}$ on $K 3$ with

$$
w_{1} \cdot S^{\prime}=1 \quad \text { and } \quad\left(w_{1}\right)^{2} \equiv 0 \bmod 4 .
$$


We also pick $w_{0} \rightarrow K 3$ with

$$
w_{0} \cdot S^{\prime}=0 \quad \text { and } \quad\left(w_{0}\right)^{2} \equiv 0 \bmod 4
$$

For example, $w_{0}=0$ will do. We now break up the argument into four cases, according to whether $\mathscr{D}^{w}$ is an even or odd power series, and according to whether $n$ (or equivalently $a$ ) is even or odd.

$\mathscr{D}^{\boldsymbol{w}}$ even and $\boldsymbol{n}$ even. Consider the surfaces

$$
\Sigma_{0}, \Sigma_{2}, \ldots, \Sigma_{a-2}, \Sigma_{a}
$$

in $\tilde{X}$. Set $\tilde{w}=w_{0}$ or $w_{1}$, chosen so that $\tilde{w} \cdot \Sigma_{r} \equiv w \cdot \Sigma \bmod 2$. According to Proposition 7.23, we have

$$
\mathscr{D}_{\tilde{X}}^{\tilde{w}}\left(t S_{r}\right)=e^{n t^{2} / 2}(\cosh t)^{r} .
$$

The power series $(\cosh t)^{r}$, for $r=0,2, \ldots, a$, span the space of even power series in $t$ modulo terms of order $t^{a+2}$. So we can find constants $\alpha_{0}, \alpha_{2}, \ldots, \alpha_{a}$ such that

$$
\mathscr{D}_{X}^{w}(t S)-\alpha_{0} \mathscr{D}_{\tilde{X}}^{\tilde{w}}\left(t S_{0}\right)-\alpha_{2} \mathscr{D}_{\tilde{X}}^{\tilde{w}}\left(t S_{2}\right)-\cdots-\alpha_{a} \mathscr{D}_{\tilde{X}}^{\tilde{w}}\left(t S_{a}\right)=O\left(t^{a+2}\right) .
$$

All the surfaces in this formula are fitting and have the same $a$ and $n$. It follows from Corollary 7.22 that the left-hand side is identically zero. That is,

$$
\mathscr{D}_{X}^{w}(t S)=e^{n t^{2} / 2}\left(\alpha_{0}+\alpha_{2}(\cosh t)^{2}+\cdots+\alpha_{a}(\cosh t)^{a}\right) .
$$

Using the multi-angle formulae this becomes

$$
\mathscr{D}_{X}^{w}(t S)=e^{n t^{2} / 2}\left(\beta_{0}+\beta_{2} \cosh (2 t)+\cdots+\beta_{a} \cosh (a t)\right),
$$

which is the assertion to be proved.

$\mathscr{D}^{\boldsymbol{w}}$ even and $\boldsymbol{n}$ odd. Now $a$ is odd and we use the surfaces

$$
\Sigma_{1}, \Sigma_{3}, \ldots, \Sigma_{a-2}, \Sigma_{a}
$$

Choose $\tilde{w}$ as before. The functions $(\cosh t)^{r}$, for $r=1,3, \ldots, a$, span the space of even power series in $t$ modulo terms of order $t^{a+1}$. There therefore exist constants $\alpha_{1}, \alpha_{3}, \ldots, \alpha_{a}$ such that

$$
\mathscr{D}_{X}^{w}(t S)-\alpha_{1} \mathscr{D}_{\tilde{X}}^{\tilde{w}}\left(t S_{1}\right)-\alpha_{3} \mathscr{D}_{\tilde{X}}^{\tilde{w}}\left(t S_{3}\right)-\cdots-\alpha_{a} \mathscr{D}_{\tilde{X}}^{\tilde{w}}\left(t S_{a}\right)=O\left(t^{a+1}\right) .
$$


Corollary 7.22 again implies that the left-hand side is identically zero. So

$$
\mathscr{D}_{X}^{w}(t S)=e^{n t^{2} / 2}\left(\alpha_{1} \cosh t+\alpha_{3}(\cosh t)^{3}+\cdots+\alpha_{a}(\cosh t)^{a}\right)
$$

which can be re-written

$$
\mathscr{D}_{X}^{w}(t S)=e^{n t^{2} / 2}\left(\beta_{1} \cosh t+\beta_{3} \cosh (3 t)+\cdots+\beta_{a} \cosh (a t)\right) .
$$

$\mathscr{D}^{\boldsymbol{w}}$ odd and $n$ even. Use the surfaces with even $r$, excluding $\Sigma_{0}$ :

$$
\Sigma_{2}, \Sigma_{4}, \ldots, \Sigma_{a-2}, \Sigma_{a}
$$

Take $\tilde{w}=w_{0}+E_{1}$ or $w_{1}+E_{1}$, chosen so that $\tilde{w} \cdot \Sigma_{r}=w \cdot \Sigma \bmod$ 2. (Note that $\tilde{w} \cdot \Sigma_{r}$ is independent of $r$ for the family above, because the case $r=0$ is excluded.) The invariant $\mathscr{D}_{\tilde{X}}^{\tilde{w}}$ is odd; according to Proposition 7.23, we have

$$
\mathscr{D}_{\tilde{X}}^{\tilde{w}}\left(t S_{r}\right)=-e^{n t^{2} / 2} \sinh t(\cosh t)^{r-1} .
$$

The functions $\sinh t(\cosh t)^{r-1}$, for $(r-1)=1,3, \ldots,(a-1)$, span the odd power series in $t$ modulo terms of order $t^{a+1}$. It follows that we have constants $\alpha_{r}$ for which

$$
\mathscr{D}_{X}^{w}(t S)-\alpha_{2} \mathscr{D}_{\tilde{X}}^{\tilde{w}}\left(t S_{2}\right)-\alpha_{3} \mathscr{D}_{\tilde{X}}^{\tilde{w}}\left(t S_{4}\right)-\cdots-\alpha_{a} \mathscr{D}_{\tilde{X}}^{\tilde{w}}\left(t S_{a}\right)=O\left(t^{a+1}\right) .
$$

Again, the left-hand side must vanish, so

$$
\begin{aligned}
\mathscr{D}_{X}^{w}(t S)=-e^{n t^{2} / 2}\left(\alpha_{2} \sinh t \cosh t\right. & +\alpha_{4} \sinh t(\cosh t)^{3}+\cdots \\
& \left.+\alpha_{a} \sinh t(\cosh t)^{a-1}\right)
\end{aligned}
$$

which can also be written

$$
\mathscr{D}_{X}^{w}(t S)=e^{n t^{2} / 2}\left(\beta_{2} \sinh (2 t)+\beta_{4} \sinh (4 t)+\cdots+\beta_{a} \sinh (a t)\right) .
$$

$\mathscr{D}^{\boldsymbol{w}}$ odd and $\boldsymbol{n}$ odd. Because $a$ is odd we have the family of surfaces with $r$ odd:

$$
\Sigma_{1}, \Sigma_{3}, \ldots, \Sigma_{a-2}, \Sigma_{a}
$$

Take $\tilde{w}=w_{0}+E_{1}$ or $w_{1}+E_{1}$ as in the previous case. The odd functions $\sinh t(\cosh t)^{r-1}$, for $r=1,3, \ldots, a$, span the space of odd power series in $t$ modulo terms of order $a+2$, and the proof is completed as before. 
This completes the proof of the Proposition under the additional hypotheses that $\Sigma \hookrightarrow X$ is fitting and $n$ is large compared to $a$ (the inequality 7.20). Consider now a surface $\Sigma \hookrightarrow X$ which does not necessarily satisfy these conditions. Let $S$ be the class it represents, and write $a=a(\Sigma)$ and $n=Q(S)$. Let $\Sigma_{m}$ be a surface representing $m S$, constructed as in Lemma 7.7. We have

$$
a\left(\Sigma_{m}\right)=m a \quad \text { and } \quad Q\left(\Sigma_{m}\right)=m^{2} a .
$$

Because the former grows linearly with $m$ and the latter grow quadratically, the surface $\Sigma_{m}$ satisfies the constraint 7.20 once $m$ is sufficiently large. The surface $\Sigma_{m}$ may or may not be fitting. If it is, define $\Sigma_{m}^{\prime}=\Sigma_{m}$. Otherwise, put $\Sigma_{m}^{\prime}=\Sigma_{m} \# T^{2}$, so that $\Sigma_{m}^{\prime}$ is fitting. We therefore have

$$
a\left(\Sigma_{m}^{\prime}\right)=m a+2 \epsilon
$$

where $\epsilon=0$ or 1 .

Choose two coprime integers $m_{1}$ and $m_{2}$, both large enough that $\Sigma_{m_{1}}^{\prime}$ and $\Sigma_{m_{2}}^{\prime}$ satisfy 7.20 , and both bigger than 2 and odd for later convenience. The Proposition has already been established for surfaces satisfying these conditions. So, using $\Sigma_{m_{1}}^{\prime}$, we have, say

$$
\mathscr{D}^{w}\left(t\left(m_{1} S\right)\right)=\exp \left(\frac{Q\left(t m_{1} S\right)}{2}\right) \sum_{r} \beta_{r} \cosh (r t),
$$

where the sum runs over integers $r$ equal to $m_{1} a \equiv a \bmod 2$ and not exceeding $m_{1} a+2 \epsilon$. Here we have taken the case that the invariants are even: the odd case is no different. We can rewrite this in the form

$$
\mathscr{D}^{w}(t S)=\exp \left(\frac{Q(t S)}{2}\right) \sum_{\rho \in \Omega_{1}} \beta_{\rho} \cosh (\rho t),
$$

where the sum now runs over rational numbers $\rho$ in the set

$$
\Omega_{1}=\left\{\rho=r / m_{1} \mid r \equiv a \bmod 2 \text { and } 0 \leq \rho \leq a+2 \epsilon / m_{1}\right\} .
$$

Using $\Sigma_{m_{2}}^{\prime}$ in just the same way, we have

$$
\mathscr{D}^{w}(t S)=\exp \left(\frac{Q(t S)}{2}\right) \sum_{\sigma \in \Omega_{2}} \gamma_{\sigma} \cosh (\sigma t)
$$


where

$$
\Omega_{2}=\left\{\sigma=r / m_{2} \mid r \equiv a \bmod 2 \text { and } 0 \leq \sigma \leq a+2 \epsilon / m_{2}\right\}
$$

From these two expressions we have

$$
\sum_{\rho \in \Omega_{1}} \beta_{\rho} \cosh (\rho t)=\sum_{\sigma \in \Omega_{2}} \gamma_{\sigma} \cosh (\sigma t) .
$$

Because the functions $\cosh (\lambda t)$ are linearly independent, there must be a non-zero term on the right-hand side to match each non-zero term on the left. It follows that $\beta_{\rho}$ can be non-zero only for $\rho$ in the set $\Omega_{1} \cap \Omega_{2}$. Since $m_{1}$ and $m_{2}$ are coprime odd integers, not less than 3, this intersection is precisely the set of integers $r$ in the range $0 \leq r \leq a$, satisfying $r \equiv a \bmod 2$. This completes the proof of the Proposition. q.e.d.

(vi) The structure theorem for fixed $w$. We shall now prove Theorem 1.7 for a fixed value of $w$. That is, we shall show that, for $X$ of simple type with $b^{1}=0$, there are constants $\beta_{r}(w)$ and classes $K_{r}$, which are integral lifts of $w_{2}(X)$, such that

$$
\mathscr{D}^{w}=\exp \left(\frac{Q}{2}\right) \sum_{r=1}^{s} \beta_{r}(w) e^{K_{r}} .
$$

We shall also establish the adjunction inequality which forms part of (b) of the Theorem. The question of how the $\beta_{r}$ and $K_{r}$ depend on $w$ will be left to the next subsection.

Proposition 7.30 can be slightly generalized without additional cost. Recalling the definition of $\mathscr{D}^{w}$ as

$$
\mathscr{D}^{w}(h)={ }^{+} D^{w}\left(e^{h}\right),
$$

we introduce temporarily the notation

$$
\mathscr{D}^{w}(h ; z)={ }^{+} D^{w}\left(e^{h} z\right),
$$

regarded as a function of $h$ (or a formal power series), with $z \in \mathbb{A}(X)$ fixed. We are particularly concerned with the power series $\mathscr{D}^{w}\left(t S ; T^{d}\right)$, for $T \in H_{2}(X ; \mathbb{R})$ a class orthogonal to $S$. In this case, because $T^{d}$ comes from $\mathbb{A}(X / \Sigma)$, the proof of (7.30) also establishes the following: 
Proposition 7.32. Let $X, \Sigma$ and $w$ be as in Proposition 7.30, let $S$ be the class represented by $\Sigma$, with $Q(S)>0$, and let $a=a(\Sigma)$. Further, let $T$ be a two-dimensional homology class orthogonal to $S$, and fix an integer $d \geq 0$. Then there exist constants $\beta_{r}$ (depending on $T$ and $d$ also) such that

$$
\mathscr{D}_{X}^{w}\left(t S ; T^{d}\right)= \begin{cases}\exp \left(\frac{Q(t S)}{2}\right) \sum_{r} \beta_{r} \cosh (r t), & \text { or } \\ \exp \left(\frac{Q(t S)}{2}\right) \sum_{r} \beta_{r} \sinh (r t), & \end{cases}
$$

according as the power series $\mathscr{D}_{X}^{w}\left(t S ; T^{d}\right)$ is even or odd. The sum extends over integers $r$ in the range $0 \leq r \leq a$ satisfying $r \equiv Q(S)$ mod 2.

Starting from this Proposition, the proof of Theorem 1.7 (for a fixed $w$ ) is a matter of elementary manipulation of formal power series. First we recast this Proposition and its predecessor in slightly different terms. Given a surface $\Sigma$ with $a(\Sigma)=a$, representing a class $S$ with $Q(S)>0$, let $P_{\Sigma}$ denote the differential operator

$$
P_{\Sigma}=e^{Q / 2} \circ\left(\prod_{r=-a}^{a}\left(\nabla_{S}-r\right)\right) \circ e^{-Q / 2},
$$

regarded as an operator on functions on $H_{2}(X)$. Here $\nabla_{S}$ is ordinary differentiation in the direction $S$ and $e^{Q / 2}$ is a multiplication operator. This operator acts also on power series, quite formally. Let $\langle S\rangle$ denote the ray spanned by $S$ in $H_{2}(X)$, and let $\left.\mathscr{D}^{w}\right|_{\langle S\rangle}$ denote the formal restriction of $\mathscr{D}^{w}$ to this ray. Then Proposition 7.30 asserts:

$$
P_{\Sigma}\left(\left.\mathscr{D}^{w}\right|_{\langle S\rangle}\right)=0 .
$$

This is a less sharp statement than the Proposition, because it makes no mention of the (trivial) fact that $\left.\mathscr{D}^{w}\right|_{\langle S\rangle}$ is either odd or even, nor of the less trivial fact that only integers $r \equiv a \bmod 2$ are needed; but this simplified form is all we need at present.

We need two elementary lemmas about these differential operators on formal power series.

Lemma 7.34. For any $T \in H_{2}(X ; \mathbb{R})$, we have

$$
\nabla_{T} \mathscr{D}^{w}=\mathscr{D}^{w}(; T) .
$$


Proof. We calculate

$$
\begin{aligned}
\nabla_{T} \mathscr{D}^{w}(S) & =\sum_{d} \nabla_{T}{ }^{+} D^{w}\left(S^{d}\right) / d ! \\
& =\sum_{d}^{d} d^{+} D^{w}\left(S^{d-1} T\right) / d ! \\
& =\sum_{d}^{+} D^{w}\left(S^{d-1} T\right) /(d-1) ! \\
& =\mathscr{D}^{w}(S ; T) . \quad \text { q.e.d. }
\end{aligned}
$$

Lemma 7.35. If $S \cdot T=0$ then $\nabla_{T}$ commutes with $P_{\Sigma}$.

Proof. Let $T^{\dagger}$ be the linear function $h \mapsto h \cdot T$ on $H_{2}(X)$. We calculate

$$
e^{-Q / 2} \circ \nabla_{T} \circ e^{Q / 2}=\nabla_{T}+T^{\dagger} .
$$

Because $S \cdot T=0$, we also have $\nabla_{S} T^{\dagger}=0$, which we can write as

$$
\left[T^{\dagger}, \nabla_{S}\right]=0 .
$$

We also have $\left[\nabla_{T}, \nabla_{S}\right]=0$ irrespective of whether $T$ is orthogonal to $S$. Thus

$$
\left[e^{-Q / 2} \circ \nabla_{T} \circ e^{Q / 2}, \Pi\left(\nabla_{S}-r\right)\right]=0,
$$

and hence $\left[\nabla_{T}, P_{\Sigma}\right]=0 . \quad$ q.e.d.

On account of the first of these two lemmas, we can recast the conclusion of Proposition 7.32 in the spirit of (7.33) by writing

$$
\left.P_{\Sigma}\left(\nabla_{T}^{d} \mathscr{D}^{w}\right)\right|_{\langle S\rangle}=0
$$

for all $T$ orthogonal to $S$ and all $d \geq 0$. On account of the second lemma, we then have

$$
\left.\left(\nabla_{T}^{d} P_{\Sigma} \mathscr{D}^{w}\right)\right|_{\langle S\rangle}=0 .
$$

So $P_{\Sigma} \mathscr{D}^{w}$ is a formal power series which vanishes on the ray $\langle S\rangle$ together with all its normal derivatives of all orders. We deduce:

Lemma 7.36. Let $X$ be a 4-manifold of simple type with $b^{1}=0$, and let $\Sigma$ be an embedded surface with $a(\Sigma)=a$, representing a class $S$ with $Q(S)>0$. Then $P_{\Sigma} \mathscr{D}^{w}=0$, for each $w$.

Proof of Theorem 1.7 for fixed $w$. Let $S_{1}, \ldots, S_{b}$ be an integral basis for $H_{2}(X ; \mathbb{Z})$ consisting of classes of positive square; here $b=b_{2}(X)$ 
therefore. Let $\Sigma_{i}$ be surfaces representing the classes $S_{i}$, and pick $w \rightarrow X$. Let $a_{i}=a\left(\Sigma_{i}\right)$, and write $\mathbf{a}=\left(a_{1}, \ldots, a_{b}\right)$. According to the last lemma, we have

$$
P_{\Sigma_{i}} \mathscr{D}^{w}=0, \quad(i=1, \ldots, b)
$$

and the general solution to this system of equations amongst formal power series is

$$
\mathscr{D}^{w}=\exp \left(\frac{Q}{2}\right) \sum_{\mathbf{r}} \beta_{\mathbf{r}} e^{K_{\mathbf{r}}}
$$

where the sum is over all $\mathbf{r}=\left(r_{1}, \ldots, r_{b}\right)$ with $\left|r_{i}\right| \leq a_{i}$, and $K_{\mathbf{r}}$ is the unique integral linear form on $H_{2}(X ; \mathbb{Z})$ satisfying

$$
K_{\mathbf{r}}\left(S_{i}\right)=r_{i} .
$$

To complete the proof of part (a) of the main theorem, it remains to show that $\beta_{\mathrm{r}}$ is zero unless $K_{\mathrm{r}}$ is an integral lift of $w_{2}(X)$. Because of Wu's formula, the latter condition equivalent to

$$
K_{\mathbf{r}}(S) \equiv Q(S) \bmod 2
$$

for all integral $S$. It is certainly enough to consider only $S$ with positive square here, and the desired result follows straight from Proposition 7.30 which describes the structure of $\mathscr{D}^{w}$ on a ray: the relevant part of that Proposition is the statement that the sum extends only over $r$ satisfying $r \equiv Q(S) \bmod 2$.

In a similar vein, Proposition 7.30 shows that $\beta_{\mathbf{r}}$ is zero unless $K_{\mathbf{r}}$ is such that

$$
a(\Sigma) \geq K_{\mathbf{r}}(S) \bmod 2
$$

for all $\Sigma$ representing classes $S$ with $Q(S)>0$. This is equivalent to the inequality in $(b)$ of Theorem 1.7, and completes the proof of parts $(a)$ and $(b)$ except for one small detail.

The remaining point is that the Theorem claims the inequality in $(b)$ holds whenever $S \neq 0$ and $Q(S) \geq 0$, whereas we have only proved it when $Q(S)$ is strictly positive. Suppose then that $Q(S)=0$. Let $T$ be an embedded surface in $X$ with $Q(T) \geq 0$ and $[T] \cdot S>0$. Such a surface exists if $S$ is non-trivial. Increasing the genus of $T$ if necessary, we may assume that $T$ meets $\Sigma$ transversely in $[T] \cdot S$ points. Let $S_{m}$ be the class $[T]+m S$, and let $\Sigma_{m}$ be a surface representing $S_{m}$, formed by taking $m$ disjoint, nearby parallel copies of $\Sigma$, and smoothing out the intersection 
points between these copies and $T$. We have $Q\left(S_{m}\right)>0$ now, and an elementary calculation shows that if $\Sigma$ violates the inequality in $(b)$ then so does $\Sigma_{m}$ once $m$ is sufficiently large, whatever the genus of $T$. The result therefore follows from the case $Q(S)$ positive, which was already established. q.e.d.

(vii) Comparing different determinants $w$. In the notation of (7.31), we are left with the question: how do the coefficients $\beta_{r}(w)$ depend on $w$ ? There is also the question of whether the set of basic classes $K_{r}$ is independent of $w$. Theorem 1.7 gives an answer: the $\beta_{r}(w)$ depend on $w$ only through a possible change of sign, and the basic classes $K_{r}$ do not depend on $w$. We now prove these assertions.

Let $S$ be once more an integral class in $H_{2}(X)$ with $Q(S)$ large and positive. Let $\Sigma$ be a fitting surface representing $S$. Fix a $w$ on $X$, and let $w^{\prime}=w-S$. Write $n=Q(S)$ and $a=a(\Sigma)$ as usual.

Lemma 7.37. There is a universal linear relation which determines the power series $\mathscr{D}^{w^{\prime}}(t S)$ in terms of $\mathscr{D}^{w}(t S)$. The relation depends only on $n, a$ and $w \cdot S \bmod 2$.

Proof. By passing to a multiple of $S$ if necessary, we may suppose that $n$ is at least 20 and that the inequality 7.20 holds. (The multiple should be odd, so that $w-S$ is still the same, mod 2.) In the proof of Proposition 7.19, we used the relations of Proposition 7.17 only in the range 7.21 :

$$
a^{+} \leq p \leq \frac{1}{2} \min \{2 g-4-(n / 2), 2 n-2\} .
$$

The condition $a<p$ ensures that the right-hand side of the relation in (7.17) is zero. Now, however, we use also the relations in the range $0 \leq p \leq a$, with $p$ satisfying the parity condition (which says that $p$ is even if $\mathscr{D}^{w}$ is even, and odd otherwise, because $\Sigma$ is fitting). With $z=1$ we obtain

$$
{ }^{+} D^{w}\left(\eta_{p}^{w}(S)\right)=(-1)^{p^{\prime}+} D^{w^{\prime}}\left(\eta_{p^{\prime}}^{w^{\prime}}(S)\right),
$$

where $p^{\prime}=a-p$. Note that $\Sigma$ will also be fitting for $w^{\prime}$ : that is, $p^{\prime}$ will have the same parity as ${ }^{+} D^{w^{\prime}}$.

Taking

$$
p^{\prime}=0,2, \ldots, 2[a / 2]
$$

in turn in the even case, or

$$
p^{\prime}=1,3, \ldots, 2[(a+1) / 2]-1
$$


in the odd case, we obtain relations which determine all coefficients of $\mathscr{D}^{w^{\prime}}(t S)$ up to (and including, if the parity is right) the term of order $t^{a}$. The remaining terms are then fixed, by Corollary 7.22. q.e.d.

We can now determine what the relation in the Lemma must be, based on the known examples supplied by $K 3$ blown up.

Lemma 7.38. In the situation of Lemma 7.37, if we write out $\mathscr{D}^{w}(t S)$ as

$$
\mathscr{D}^{w}(t S)=\exp \left(\frac{Q(t S)}{2}\right) \sum_{r} \beta_{r} e^{r t}
$$

the sum running over integers $r$ in the range $-a \leq r \leq a$ satisfying $r \equiv a \equiv n \bmod 2$, then

$$
\mathscr{D}^{w^{\prime}}(t S)=\exp \left(\frac{Q(t S)}{2}\right) \sum_{r}(-1)^{w \cdot S}(-1)^{(n-r) / 2} \beta_{r} e^{r t} .
$$

Proof. Recall the notation used in the proof of Proposition 7.30, and the examples of embedded surfaces $\Sigma_{r}$ in $\tilde{X}=K 3 \# N\left(\overline{\mathbb{C P}}^{2}\right)$ used there: we again take

$$
\Sigma_{r}=\Sigma^{\prime} \#\left(\bar{C}_{1} \# \cdots \# \bar{C}_{r}\right) \#\left(\frac{1}{2}(a-r) T^{2}\right),
$$

for $r \equiv a \bmod 2$, representing the class

$$
S_{r}=S^{\prime}-E_{1}-\cdots-E_{r},
$$

where $S^{\prime}$ is a class in $K 3$ with square $n+r$, and $\Sigma^{\prime}$ is a tight surface representing it. Each $\Sigma_{r}$ has self-intersection $n$ and $a\left(\Sigma_{r}\right)=a$. Let $w_{*}$ be an arbitrary line-bundle on $K 3$, which may have even or odd degree on $S^{\prime}$.

In proving Proposition 7.30, we saw that, for any $(X, \Sigma)$ and $w$, the function $\mathscr{D}_{X}^{w}(t S)$ could be written as a linear combination of the functions $\mathscr{D}_{\tilde{X}}^{\tilde{w}}\left(t S_{r}\right)$, for a suitable $\tilde{w}$ : we took $\tilde{w}=w_{*}$ or $w_{*}+E_{1}$ according as $\mathscr{D}_{X}^{w}$ was even or odd respectively, and $w_{*}$ was chosen so that $\tilde{w} \cdot S_{r}=w \cdot S \bmod 2$. Since the relation in Lemma 7.37 is linear, we therefore need only verify that the formula for $\mathscr{D}^{w^{\prime}}(t S)$ is correct in the case that $X$ is $K 3$ blown up, $\Sigma=\Sigma_{r}$, and $w=w_{*}$ or $w_{*}+E_{1}$. We treat the two cases in turn.

The case $\boldsymbol{w}=\boldsymbol{w}_{*}$. In this case, when $S=S_{r}$, we have

$$
w^{\prime}=\left(w_{*}-S^{\prime}\right)+E_{1}+\cdots+E_{r} .
$$


From Proposition 7.23 we obtain:

$$
\begin{aligned}
\mathscr{D}^{w}(t S) & =(-1)^{w_{*}^{2} / 2} e^{n t^{2} / 2}(\cosh t)^{r} \\
& =(-1)^{w_{*}^{2} / 2} e^{n t^{2} / 2} 2^{-r} \sum_{s}\left(\begin{array}{l}
r \\
s
\end{array}\right) e^{(r-2 s) t} \\
\mathscr{D}^{w^{\prime}}(t S) & =(-1)^{\left(w_{*}-S^{\prime}\right)^{2} / 2} e^{n t^{2} / 2}(\sinh t)^{r} \\
& =(-1)^{\left(w_{*}-S^{\prime}\right)^{2} / 2} e^{n t^{2} / 2} 2^{-r}(-1)^{r} \sum_{s}\left(\begin{array}{l}
r \\
s
\end{array}\right)(-1)^{s} e^{(r-2 s) t}
\end{aligned}
$$

The rule of transformation is therefore that $\beta_{r} e^{r t}$ be replaced with

$$
\begin{aligned}
(-1)^{w_{*}^{2} / 2}(-1)^{\left(w_{*}-S^{\prime}\right)^{2} / 2}(-1)^{r} \beta_{r} e^{r t} & =(-1)^{\left(-2 w_{*} \cdot S^{\prime}+\left(S^{\prime}\right)^{2}\right) / 2}(-1)^{r} \beta_{r} e^{r t} \\
& =(-1)^{w \cdot S}(-1)^{(n-r) / 2} \beta_{r} e^{r t}
\end{aligned}
$$

which is as asserted in the Lemma.

The case $w=w_{*}+E_{1}$. In this case

$$
w^{\prime}=w_{*}+2 E_{1}+E_{2}+\cdots+E_{r}
$$

We have

$$
\mathscr{D}^{w^{\prime}}=-\mathscr{D}^{w_{*}+E_{2}+\cdots E_{r}}
$$

and the latter is what is given by Proposition 7.23. We obtain

$$
\begin{aligned}
\mathscr{D}^{w}(t S) & =-(-1)^{w_{*}^{2} / 2} e^{n t^{2} / 2} \sinh t(\cosh t)^{r-1} \\
& =-(-1)^{w_{*}^{2} / 2} e^{n t^{2} / 2} 2^{-r} \sum_{s}\left(\begin{array}{c}
r-2 \\
s-1
\end{array}\right) e^{(r-2 s) t} \\
\mathscr{D}^{w^{\prime}}(t S) & =(-1)^{\left(w_{*}-S^{\prime}\right)^{2} / 2} e^{n t^{2} / 2} \cosh t(-\sinh t)^{r-1} \\
& =(-1)^{\left(w_{*}-S^{\prime}\right)^{2} / 2} e^{n t^{2} / 2} 2^{-r}(-1)^{r-1} \sum_{s}\left(\begin{array}{l}
r-2 \\
s-1
\end{array}\right)(-1)^{s} e^{(r-2 s) t} .
\end{aligned}
$$

The rule of transformation is therefore that $\beta_{r} e^{r t}$ be replaced with

$$
\begin{aligned}
(-1)^{w_{*}^{2} / 2}(-1)^{\left(w_{*}-S^{\prime}\right)^{2} / 2}(-1)^{r-1} \beta_{r} e^{r t} & =-(-1)^{\left(-2 w_{*} \cdot S^{\prime}+\left(S^{\prime}\right)^{2}\right) / 2} \beta_{r} e^{r t} \\
& =(-1)^{w \cdot S}(-1)^{(n-r) / 2} \beta_{r} e^{r t}
\end{aligned}
$$

which is again as asserted in the Lemma. q.e.d. 
Proof of Theorem 1.7, completion Let $w$ and $w^{\prime}$ be two different line-bundles on $X$, and let the corresponding invariants be

$$
\begin{aligned}
& \mathscr{D}^{w}=\exp \left(\frac{Q}{2}\right) \sum_{r=1}^{s} \beta_{r} e^{K_{r}} \\
& \mathscr{D}^{w^{\prime}}=\exp \left(\frac{Q}{2}\right) \sum_{r=1}^{s^{\prime}} \beta_{r}^{\prime} e^{K_{r}^{\prime}} .
\end{aligned}
$$

We suppose these are written without redundancy, so that the coefficients $\beta_{r}$ and $\beta_{r}^{\prime}$ are non-zero, the $K_{r}$ are distinct, and so are the $K_{r}^{\prime}$. Write $c_{1}\left(w^{\prime}\right)=c_{1}(w)-\delta$, and choose a homology class $S \in H_{2}(X ; \mathbb{Z})$ with $Q(S)$ positive, satisfying $S \equiv$ P.D. $(\delta) \bmod 4$. This ensures that $\mathscr{D}^{w^{\prime}}=\mathscr{D}^{w-\text { P.D. }(S)}$. Write $Q(S)=n$, and let $\Sigma$ be any fitting surface representing $S$. Applying the previous Lemma to this situation, we find that

$$
\begin{aligned}
\mathscr{D}^{w^{\prime}}(t S) & =e^{n t^{2} / 2} \sum_{r=1}^{s}(-1)^{w \cdot S}(-1)^{\left(n-K_{r} \cdot S\right) / 2} \beta_{r} e^{\left(K_{r} \cdot S\right) t} \\
& =e^{n t^{2} / 2} \sum_{r=1}^{s}(-1)^{w \cdot \delta}(-1)^{\left(\delta^{2}-K_{r} \cdot \delta\right) / 2} \beta_{r} e^{\left(K_{r} \cdot S\right) t}
\end{aligned}
$$

It follows that

$$
\sum_{r=1}^{s^{\prime}} \beta_{r}^{\prime} e^{\left(K_{r}^{\prime} \cdot S\right) t}=\sum_{r=1}^{s}(-1)^{w \cdot \delta}(-1)^{\left(\delta^{2}-K_{r} \cdot \delta\right) / 2} \beta_{r} e^{\left(K_{r} \cdot S\right) t}
$$

As this holds for all $S \equiv$ P.D. $(\delta) \bmod 4$, it follows that the expressions are equal term by term, and that after reordering, we have $K_{r}=K_{r}^{\prime}$; and, switching notation so as to write $\beta_{r}\left(w^{\prime}\right)$ for $\beta_{r}^{\prime}$ :

$$
\beta_{r}\left(w^{\prime}\right)=(-1)^{w \cdot \delta}(-1)^{\left(\delta^{2}-K_{r} \cdot \delta\right) / 2} \beta_{r}(w)
$$

This we can rewrite as

$$
(-1)^{\left(\left(w^{\prime}\right)^{2}+K_{r} \cdot w^{\prime}\right) / 2} \beta_{r}\left(w^{\prime}\right)=(-1)^{\left(w^{2}+K_{r} \cdot w\right) / 2} \beta_{r}(w)
$$

which shows that the dependence of $\beta_{r}(w)$ on $w$ is as claimed in Theorem 1.7. q.e.d. 


\section{The simple type condition.}

(i) Tight surfaces and simple type. In this section we describe some sufficient conditions for a 4-manifold $X$ to have simple type. Recall that an oriented, embedded surface $\Sigma$ representing a homology class $S$ in $X$ is tight if 2 genus $(\Sigma)-2=Q(S)$.

Theorem 8.1. An admissible 4-manifold $X$ with $b_{1}(X)=0$ has simple type if it contains a tight embedded surface of genus 2 or more.

Proof. The genus of $\Sigma$ being at least 2 means that the selfintersection number of the class $S$ which $\Sigma$ represents is positive. Because of Lemma 7.7, there is no loss of generality in assuming that $S$ is divisible by 2 . In this case, the self-intersection number $n=Q(S)$ is divisible by 4 and the genus is odd. We can also make the genus of $\Sigma$ and $n$ as large as we wish, using the same lemma. We will need $n$ to be at least 20 .

Pick any $w \rightarrow X$. The singular invariants $D_{X, \Sigma}^{w, p}$ are defined for $p$ even, because $(g-1)$ and $w(S)$ are even (2.20); and as long as $n$ is at least 20 we can apply the basic relations of Theorem 7.4 with $p=4$ to obtain

$$
D^{w}\left(\omega_{4}^{w} z\right)=0
$$

for all $z \in \mathbb{A}(X / \Sigma)$. (We omit the maps $\imath_{*}$ and $\jmath_{*}$ from our notation again.) Now recall the partial calculation of $\omega_{4}^{w}$ which we made in Proposition 6.4. We have

$$
\begin{aligned}
\omega_{4}^{w} & =\omega_{4,0}^{w}+\omega_{4,1}^{w} \\
& =\frac{1}{24}\left(S^{4}-(6 g-10) x S^{2}+3(g-1)(g-3) x^{2}-4 S \Gamma\right)+F^{w}(g, n) .
\end{aligned}
$$

In our case, $g$ is $\frac{1}{2} n+1$ and $\Gamma$ makes no contribution, since $b_{1}(X)$ is zero. Thus

$$
D^{w}\left(S^{4} z-(3 n-4) x S^{2} z+\frac{3}{4}\left(n^{2}-4 n\right) x^{2} z+24 F^{w}\left(\frac{1}{2} n+1, n\right) z\right)=0
$$

The function $F^{w}\left(\frac{1}{2} n+1, n\right)$ is a universal function of $n$. We can work out what it must be from the one example we understand well, namely the $K 3$ surface. From this example we obtain, with $z=1$,

$$
3 n^{2}-(3 n-4)(2 n)+3\left(n^{2}-4 n\right)+24 F^{w}\left(\frac{1}{2} n+1, n\right)=0,
$$


so

$$
F^{w}\left(\frac{1}{2} n+1, n\right)=n / 6 \text {. }
$$

The universal relation (8.2) therefore reduces to

$$
D^{w}\left(S^{4} z-(3 n-4) x S^{2} z+\frac{3}{4}\left(n^{2}-4 n\right) x^{2} z+4 n z\right)=0 .
$$

As this relation is universal, we can apply it to the class $k S$ in $H_{2}(X ; \mathbb{Z})$, which, like $S$, is represented by a tight surface of large genus, for any positive integer $k$. The self-intersection number is $k^{2} n$, so we obtain

$$
D^{w}\left(k^{4} S^{4} z-k^{2}\left(3 k^{2} n-4\right) x S^{2} z+\frac{3}{4} k^{2}\left(k^{2} n^{2}-4 n\right) x^{2} z+4 k^{2} n z\right)=0 .
$$

This identity has parts involving $k^{2}$ and $k^{4}$, and we can equate both of these to zero, because the identity holds for all $k$. From the $k^{4}$ terms we obtain

$$
D^{w}\left(S^{4} z-3 n x S^{2} z+\frac{3}{4} n^{2} x^{2} z\right)=0,
$$

and from the $k^{2}$ terms we obtain

$$
D^{w}\left(4 x S^{2} z-3 n x^{2} z+4 n z\right)=0 .
$$

We now use also the relation with $p=2$, namely $D^{w, 2}=0$, as in Lemma 7.6(b). Applied to the class $x z \in \mathbb{A}(X / \Sigma)$, this gives

$$
D^{w}\left(S^{2} x z-(n / 2) x^{2} z\right)=0 .
$$

Cancelling the $S^{2}$ term between these last two equations and dividing by $n$ gives

$$
D^{w}\left(x^{2} z\right)=4 D^{w}(z) .
$$

This equation is the defining condition for simple type, but we have established it only when $z \in \mathbb{A}(X / \Sigma)$. To conclude the proof, we now show by induction on $p$ that

$$
D^{w}\left(x^{2} S^{p} z\right)=4 D^{w}\left(S^{p} z\right)
$$

We treat first the case of even $p$, the case $p=0$ having been already established. For this, we use the recurrence relations, in the shape of the formula

$$
D^{w}\left(\omega_{p}^{w} z\right)=0
$$


This allows us to write

$$
D^{w}\left(S^{p} z\right)=\sum_{i>0} a_{i} D^{w}\left(S^{p-2 i} x^{i} z\right)
$$

In this universal formula, we can also substitute $x^{2} z$ for $z$ to obtain

$$
D^{w}\left(S^{p} x^{2} z\right)=\sum_{i>0} a_{i} D^{w}\left(S^{p-2 i} x^{i+2} z\right)
$$

If the formula (8.3) is known to hold for all smaller, even $p$, then we can apply (8.3) with $x^{i} z$ in place of $z$ to obtain

$$
\begin{aligned}
D^{w}\left(S^{p} x^{2} z\right) & =\sum_{i>0} 4 a_{i} D^{w}\left(S^{p-2 i} x^{i} z\right) \\
& =4 D^{w}\left(S^{p} z\right)
\end{aligned}
$$

as required. As it stands this argument is valid only for $p$ in a certain range, because the universal relation (8.4) is valid only for $2 p \leq \Delta(n, g)$. The by now familiar device of replacing $S$ by $k S$ allows one to continue the induction for all $p$.

For the odd case, consider a surface $\Sigma^{\prime}=\Sigma \# T^{2}$ with $a\left(\Sigma^{\prime}\right)=2$, still representing the same class $S$. Since $g-1-w(S)$ is now odd, we can apply the relations with $p=1$ to obtain

$$
D^{w}\left(\omega_{1}^{w} z\right)=-D^{w}\left(\omega_{1}^{w} z\right)
$$

and hence, since $\omega_{1}^{w}=S$,

$$
D^{w}(S z)=0 \text {. }
$$

One can now prove by induction that $D^{w}\left(S^{p} z\right)=0$ for all odd $p$, using the relations $D^{w}\left(\omega_{p}^{w} z\right)=0$ for odd $p>1$, applied to the pair $\left(X, \Sigma^{\prime}\right)$. Again, when $p$ becomes large, one must replace $S$ by $k S$ and take a surface with $a=2$ representing this multiple. q.e.d.

Corollary 8.5. A 4-manifold $X$ with $b_{1}=0$ and $b^{+}>1$ has simple type if it contains a sphere of square -2 meeting a torus of square 0 transversely in one positive intersection point.

Proof. Take two disjoint, parallel copies of the torus and smooth their intersection points with the sphere to obtain a tight surface of genus 2 . q.e.d. 
Remark. In section 7(ii), we showed that $K 3$ had simple type using an argument which was rather simpler than the proof of Theorem 8.1 given above, in that the main mechanism was the relation of degree 2 in $S$, rather than the degree 4 relation which fuelled the argument here. An examination of the argument used in 7(ii) reveals that it depends on having a tight surface with $(g-1)-w(S)$ odd. This makes the argument less general: the proof of Theorem 8.1 used a tight surface with $(g-1)-w(S)$ even, and such a surface can always be constructed from an odd one by replacing $S$ by $2 S$.

(ii) Examples. Tight surfaces can arise naturally as totally real submanifolds of complex surfaces, and this leads to one criterion for simple type:

Lemma 8.6. Let $X$ be a smooth, 2-dimensional variety defined over $\mathbb{R}$. Suppose the real locus $X(\mathbb{R})$ has a component which is a closed, orientable surface. Then that component is a tight surface, regarded as a submanifold of the complex locus $X(\mathbb{C})$ with its standard complex orientation.

Proof. The complex structure $I$, thought of as an endomorphism of the tangent bundle of the 4-manifold $X(\mathbb{C})$, carries the tangent bundle of $X(\mathbb{R})$ onto the normal bundle. If $\left(v_{1}, v_{2}\right)$ is an oriented frame for the tangent space to $X(\mathbb{R})$ at a point, then $\left(I v_{1}, I v_{2}\right)$ is a frame for the normal bundle, but $\left(v_{1}, v_{2}, I v_{1}, I v_{2}\right)$ is not a correctly oriented frame for the 4-dimensional tangent space with the standard complex orientation. Therefore the normal bundle is isomorphic to the cotangent bundle in an orientation-preserving way. The degree of the normal bundle to an orientable component of genus $g$ is therefore $2 g-2$, which shows that the component is tight. q.e.d.

Corollary 8.7. $\quad$ Let $X$ be a smooth projective algebraic surface defined over $\mathbb{R}$ whose real locus $X(\mathbb{R})$ contains an orientable component of genus 2 or more. Suppose the complex surface $X(\mathbb{C})$ has $b_{1}=0$ and $b^{+}>1$ (or equivalently $q=0$ and $p_{g}>0$ ). Then $X(\mathbb{C})$ has simple type.

Proof. Combine the Lemma above with Theorem 8.1. $\quad$ q.e.d. Similar considerations give:

Proposition 8.8. Let $X$ be a symplectic 4-manifold with $b_{1}=0$ and $b^{+}>1$. Suppose $X$ contains an orientable Lagrangian surface of genus 2 or more. Then $X$ has simple type.

Lemma 8.9. Let $Y$ be a smooth projective 3 -fold with $b_{1}=0$, and 
let $X_{d}$ be a smooth surface in $Y$ belonging to the linear system $|d H|$, where $H$ is the hyperplane class. Then $X_{d}$ has simple type if $d \geq 4$.

Proof. Choose homogeneous coordinates for the ambient projective space $\mathbb{C P}^{N}$ in such a way that the codimension-three space $K=\left\{X_{1}=X_{2}=X_{3}=0\right\}$ meets $Y$ transversely in isolated points. When $d$ is at least 4 , let $S_{d}$ be a singular hypersurface in $\mathbb{C P}^{N}$ one of whose components is the quartic

$$
X_{1}^{4}+X_{2}^{4}+X_{3}^{4}=0
$$

and whose other components (if $d>4$ ) are well away from the isolated points $Y \cap K$. Now deform this (possible reducible) hypersurface $S_{d}$ slightly so that it becomes smooth and meets $Y$ transversely. Let $X_{d}$ be the intersection. Near each of the points of $Y$ which meets the locus $K$, the new $X_{d}$ will contain a $C^{\infty}$ copy of the Milnor fibre of the singularity (8.10), regarded now as an affine quartic in $\mathbb{C}^{3}$. To conclude the proof we should find a tight surface of large genus in this Milnor fibre, and then apply Theorem 8.1. One way to find a tight surface is to use Lemma 8.6. A suitable real form of the Milnor fibre is the smooth quartic surface

$$
\left(X_{1}^{2}-3\right)^{2}+\left(X_{2}^{2}-3\right)^{2}+\left(X_{3}^{2}-3\right)^{2}=16,
$$

whose real locus is a compact, orientable surface of genus 5 . (The genus5 handlebody which this encloses in $\mathbb{R}^{3}$ looks like a much-thickened 1-skeleton of a standard cube.) q.e.d.

Lemma 8.11. Let $Y$ be a smooth projective 5-fold and $X_{p, q, r} a$ surface in $Y$ formed as the transverse intersection with three hypersurface in $\mathbb{C P}^{N}$ of degrees $p, q$ and $r$, all at least 2 . Then $X_{p, q, r}$ has simple type.

Proof. The same argument as in the previous lemma reduces one to considering a 2-dimensional surface singularity $Z$ formed as the complete intersection of three homogeneous quadratic cones in $\mathbb{C}^{5}$, and its Milnor fibre $Z_{\epsilon}$. We must find a tight surface of genus 2 or more in $Z_{\epsilon}$. Seeking to apply Lemma 8.6 again, we are led to seek a suitable real form of $Z_{\epsilon}$. Consider a 2-dimensional plane $\Pi$ in $\mathbb{R}^{5}$, so positioned that its intersection with the 'octant' in which all 5 coordinates are non-negative is a (compact) pentagon. Take the surface in $\mathbb{R}^{5}$ defined by the condition

$$
\left(x_{1}^{2}, x_{2}^{2}, x_{3}^{2}, x_{4}^{2}, x_{5}^{2}\right) \in \Pi .
$$


This is a complete intersection of three quadrics, so it is a real form of some $Z_{\epsilon}$. It is a closed, orientable surface, and the coordinate hyperplanes divide it into 32 pentagons, which tessellate the surface, with 4 pentagons meeting at each vertex. The genus of the surface is therefore 5. q.e.d.

Corollary 8.12. If $X$ is a smooth complete intersection of general type in $\mathbb{C P}^{N}$, then $X$ has simple type.

Proof. If $N$ is at least 5, then Lemma 8.11 applies. The remaining cases all come under Lemma 8.9, with one exception: the intersection of two cubics in $\mathbb{C P} 4$. To deal with this case, let $S$ be a smooth, real cubic surface in $\mathbb{R} P^{3}$ of negative Euler characteristic, such as the projective completion of the affine surface

$$
x^{3}+y^{3}+z^{3}=x+y+z .
$$

Let $C(S)$ be the cone on $S$, sitting in $\mathbb{R}^{4}$. The intersection of $C(S)$ with the unit sphere $S^{3}$ is the orientable double-cover of $S$. This exhibits an orientable surface of negative Euler characteristic as a complete intersection of a cubic and a quadric. The same surface arises as a component of the complete intersection of two cubics: one only has to make one of the cubics reducible. Now apply Corollary 8.7. $\quad$ q.e.d.

Various branched coverings can be handled in a very similar manner. For example:

Proposition 8.13. Let $Z$ be a smooth complex projective surface with $b_{1}=0$, and let $C_{p} \subset Z$ be a smooth curve in the linear system $|2 p H|$. Let $X_{p}$ be the branched double-cover of $Z$, branched along $C_{p}$. Then $X_{p}$ has simple type for all $p \geq 3$.

Proof. Along the same lines as the previous propositions, we can consider the affine surface $X$ which is the branched double-cover of $\mathbb{C}^{2}$, branched along the Milnor fibre of the singularity $x^{6}+y^{6}=0$. All $X_{p}$ for $p \geq 3$ contain a copy of this $X$, so we need only find a tight surface of genus 2 or more in this space. If one deforms the plane singularity to obtain a real, plane sextic consisting of three ovals, two of them inside a third, then the real locus of the double covering (for a suitable lift of the real involution) consists of an orientable surface of genus 2: it arises as the doubling of the disk with two holes which lies between the three ovals. q.e.d.

Proposition 8.14. Regular elliptic surfaces with $b^{+}>1$ have simple type. 
Proof. Using the non-standard nuclei of [19], one can find inside an elliptic $K 3$ surface a configuration consisting of a sphere and a torus, of the sort required by Corollary 8.5, entirely disjoint from one elliptic fibre $F$. Without disturbing the configuration, one can alter the $K 3$ surface, either by performing logarithmic transforms in the neighbourhood of $F$ or by forming a fibre sum with copies of the rational elliptic surface. According to [17], every regular elliptic surface is diffeomorphic to one of the manifolds obtained in this way. They all have simple type by Corollary 8.5. q.e.d.

(iii) Discussion. The examples given above do not constitute strong evidence for the conjecture that all 4-manifolds with $b_{1}=0$ and $b^{+}>1$ (or even all regular complex surfaces with $p_{g}>0$ ) have simple type. Although the authors have no counter-example to such a conjecture, the cases which are understood are too special to give confidence.

All the cases treated above can be seen to have simple type on account of having a tight embedded surface of positive square, using Theorem 8.1. It is therefore sobering to observe that there is at least one family of simply-connected 4-manifolds with $b^{+}>1$ which contain no such surfaces: examples are provided by the non-complex, fake $K 3$ surfaces of [19] and their non-spin companions, in the case that logarithmic transforms are performed on three disjoint nuclei. A 4-manifold $X$ of this sort does in fact have simple type: there is an elementary argument using Floer homology, starting from the fact that the elliptic surfaces have simple type. One can also calculate the basic classes. For example, if one performs a single logarithmic transform of multiplicity 2 in each nucleus, and if the cohomology classes dual to the resulting multiple fibres are $\kappa_{1}, \kappa_{2}$ and $\kappa_{3}$, then there are 8 basic classes given as $\pm \kappa_{1} \pm \kappa_{2} \pm \kappa_{3}$. Using Theorem 1.7, the assertion that $X$ contains no tight surfaces of positive square is easily verified as a consequence of the fact that any class of positive square in $H_{2}(X)$ has non-zero pairing with at least one of the basic classes.

Corollary 8.7, of course, can be applied to many more surfaces than complete intersections and branched double-covers. It seems quite likely that minimal surfaces of general type satisfying the hypotheses of Corollary 8.7 are as widespread, at least with regard to their characteristic numbers $c_{1}^{2}$ and $c_{2}$, as regular surfaces in general. 


\section{Other issues}

In the final section of the paper, we take up some topics which were touched on in the introduction. We give first a proof of the 'blow-up formula' for the invariants of $X \# \overline{\mathbb{C P}}^{2}$ when $X$ has simple type. Then we discuss the relationship between the results of this paper, particularly the lower bound for the genus of embedded surfaces in part (b) of Theorem 1.7, and the earlier results of [[24],[25]]. After clarifying this relationship, we are able to use an observation of Brussee [6] to give a sufficient criterion for the canonical class of a complex surface to be one of the basic classes. We also mention various open questions in the course of the discussion.

(i) The blow-up formula for manifolds of simple type. We give here a proof of Proposition 1.9. Since the publication of the announcement [28], Fintushel and Stern have provided a simpler proof of a more general result [13], so we keep our treatment brief. Let $X, \tilde{X}$ and $E$ be as in the proposition. Pick an integer $d \geq 0$, and consider the invariants ${ }^{+} D_{\tilde{X}}^{w}\left(E^{d} z\right)$ and ${ }^{+} D_{\tilde{X}}^{w+E}\left(E^{d} z\right)$, for $z \in \mathbb{A}(X) \subset \mathbb{A}(\tilde{X})$. The content of the proposition is the statement that

$$
\begin{aligned}
{ }^{+} D_{\tilde{X}}^{w}\left(E^{d} z\right) & =a_{d}{ }^{+} D_{X}^{w}(z) \\
\text { and } \quad{ }^{+} D_{\tilde{X}}^{w+E}\left(E^{d} z\right) & =b_{d}{ }^{+} D_{X}^{w}(z),
\end{aligned}
$$

where $a_{d}$ and $b_{d}$ are the values that occur for $K 3$ (so $a_{d}$ and $b_{d}$ are the $d$ th derivatives at $t=0$ of the functions $e^{-t^{2} / 2} \cosh t$ and $-e^{-t^{2} / 2} \sinh t$ respectively).

Pick a surface $\Sigma \subset X$ representing a homology class $S$. Let $n$ and $g$ be the self-intersection number and genus, and let these be large in comparison to $a(\Sigma)$ and $d$, so that $2 d \leq \Delta(n, g)$ and the inequality 7.20 holds. For definiteness, let us arrange (as we may) that $n$ is even, $g$ is odd and $w$ is even on $S$. Let $X^{o}$ and $W^{\circ}$ be the complement of $\Sigma$ and the tubular neighbourhood of $\Sigma$ respectively, with cylindrical ends, and let $w$ also denote the cylindrical-end bundle obtained by restricting $w$ to $X^{o}$ and $W^{o}$. On the blow-up $\tilde{X}=X \# \overline{\mathbb{C P}}^{2}$, let $\tilde{w}$ be $w$ or $w+E$, according as $d$ is even or odd respectively.

Lemma 9.2. In the situation described above, there is a polynomial $\zeta(S)$, depending on $d, n, g$ and the value of $w \cdot S$ mod 2 but otherwise 
independent of $X$ and $\Sigma$, such that

$$
{ }^{+} D_{\tilde{X}}^{\tilde{w}}\left(E^{d} z\right)={ }^{+} D_{X}^{w}(\zeta(S) z)
$$

for all $z \in \mathbb{A}(X / \Sigma)$. With the above assumptions about the parity of $n$ and $g$, we can assume that $\zeta$ is an even polynomial of degree at most $a(\Sigma)$.

Proof. Let $\tilde{W}^{o}$ be the connected sum $W^{o} \# \overline{\mathbb{C P}}^{2}$, with a metric with long neck for the connected sum. This manifold has a relative invariant, which is a map

$$
D_{\tilde{W}^{\circ}}^{\tilde{w}}: \mathbb{A}\left(\tilde{W}^{o}\right)_{(\Delta)} \longrightarrow \bar{F}^{w}(Y) .
$$

The possibility of defining such an invariant, satisfying a pairing formula similar to Theorem 4.28, was mentioned as Remark (c) at the end of section 4. (Note that the definition of the invariants, as always, may involve a second blow-up, which is hidden in our notation.) The proof of the vanishing theorem for the odd components (5.4) adapts to this manifold also, so $D_{\tilde{W}^{\circ}}^{\tilde{w}}$ takes values in $\bar{F}_{\mathrm{ev}}^{w}(Y)$. The surjectivity of $D_{W^{\circ}}^{w}$ established in (5.7) means that there is a class $\gamma \in \mathbb{A}(\Sigma)$ such that

$$
D_{\tilde{W}^{\circ}}^{\tilde{w}}\left(E^{d}\right)=D_{W^{\circ}}^{w}(\gamma)
$$

Using the pairing formulae, this gives

$$
D_{\tilde{X}}^{\tilde{w}}\left(E^{d} z\right)=D_{X}^{w}(\gamma z)
$$

Let $\zeta(S)$ be the polynomial in $S$ which is equal to $\gamma$ modulo the ideal generated by $(x-2)$ and the odd-dimensional classes in $\mathbb{A}(\Sigma)$. Then the simple type condition and the hypothesis $b^{1}(X)=0$ mean that we can reduce the above relation to

$$
{ }^{+} D_{\tilde{X}}^{\tilde{w}}\left(E^{d} z\right)={ }^{+} D_{X}^{w}(\zeta(S) z) .
$$

The assumption that $d$ has the same parity as $\tilde{w} \cdot E$ means that the class $D_{\tilde{W}^{\circ}}^{\tilde{w}}\left(E^{d}\right)$ has dimension $0 \bmod 4$, so $\zeta$ will be an even polynomial. Using the universal relations, in the form of Proposition 7.19, we can reduce $\zeta$ to a polynomial of degree at most $a(\Sigma)$. (Note that $a(\Sigma)$ is even.) q.e.d.

To complete the argument, we need to show that $\zeta$ has degree zero and is equal to the constant $a_{d}$ or $b_{d}$, according as $\tilde{w}$ is $w$ or $w+E$ 
respectively. Let us deal with the former case, where $d$ is even and $\tilde{w}=w$. The number of coefficients in $\zeta$ is $1+a(\Sigma) / 2$. So we can determine $\zeta$ if we have this many (independent) inhomogeneous linear constraints. To this end, let $X$ be a $K 3$ surface blown up at $N$ points (where $N$ is any number greater than $a=a(\Sigma)$ ), let $w \rightarrow X$ be the trivial bundle, and let

$$
\Sigma_{0}, \Sigma_{2}, \ldots, \Sigma_{a-2}, \Sigma_{a}
$$

be surfaces in $X$, as described in section $7(\mathrm{v})$. These all have the same genus $g$ and self-intersection number $n$; the surface $\Sigma_{r}$ meets the first $r$ of the exceptional curves. Proposition 7.23 gives the invariants explicitly for both $X$ and $\tilde{X}$ in this case. The number of surfaces here is equal to the number of constraints needed. We leave the reader to check that the resulting constraints on $\zeta$ are independent and have the solution $\zeta(S)=a_{d}$. The odd case is similar. q.e.d.

(ii) Comparison with other obstructions. Part (b) of Theorem 1.7 provides an obstruction to embedding surfaces in $X$, by establishing a lower bound for the genus in each homology class of positive square. In [25], a slightly different obstruction was identified, also using singular connections. Here we compare the two results.

Let $(X, \Sigma)$ be an admissible pair, and write $g$ for the genus and $n$ for the self-intersection number of the class $S$ represented by $\Sigma$, as usual. Introduce a formal parameter $s$ and consider the series

$$
R(s)=\sum_{p} s^{p} D_{X, \Sigma}^{w, p}
$$

which we regard as a polynomial in $s$ taking values in the linear functions on $\mathbb{A}(X / \Sigma)$. Essentially the same series was considered in [25]. We have normalized the powers of $s$ slightly differently here, and we differ from [25] in regarding $R(s)$ as a function on $\mathbb{A}(X / \Sigma)$ rather than $\mathbb{A}(X)$. Neither of these points is of particular importance for our discussion. Note that with our present conventions, the sum extends only over $p$ satisfying the parity condition $(2.20)$, in the range $0 \leq p \leq a(\Sigma)$. In the simple type case, we can define similarly

$$
{ }^{+} R(s)=\sum_{p} s^{p+} D_{X, \Sigma}^{w, p} .
$$


Such a variant was also considered in [25] (section 5(ii)). For definiteness, let us suppose that the polynomial invariants of $X$ have even degree, that $g$ is odd, that $(g-1)-w \cdot S$ (and hence $p$ ) is even and that $n$ is even. The range of summation then includes $p=0$ and $p=a$.

Lemma 9.3. Suppose $X$ has simple type with $b^{1}(X)=0$, and that the above parity conditions hold. Suppose that the Donaldson invariants of $X$ are non-zero. Suppose further that $a=a(\Sigma)$ is small compared to $n$ and $g$, so that

$$
2 a \leq \Delta(n, g) \text {. }
$$

Then the value of ${ }^{+} R(s)$ at $s=1$ is non-zero if and only if there is a basic class $K$ such that

$$
a(\Sigma)=K \cdot S
$$

More generally, the order of vanishing of ${ }^{+} R(s)$ at $s=1$ is given by

$$
\operatorname{ord}_{s=1}{ }^{+} R(s)=\min _{K} a(\Sigma)-K \cdot S,
$$

where the minimum is taken over all basic classes.

Proof. The universal formulae, in the form of (7.17), hold for all $p$ in the range of summation, so if we put

$$
\eta^{w}(s, S)=\sum_{0 \leq p \leq a} s^{p} \eta_{p}^{w}(S)
$$

then we have a universal formula for ${ }^{+} R(s)$ :

$$
{ }^{+} R(s)(z)={ }^{+} D^{w}\left(\eta^{w}(s, S) z\right) .
$$

Note that the coefficient of $s^{a} S^{a}$ in $\eta^{w}(s, S)$ is $1 / a$ !, and that $S^{a}$ does not appear with any lower power of $s$. From this we deduce that $\eta^{w}(1, S)$ is non-zero in $\mathbb{R}[S]$.

Proposition 7.32 can be recast as saying that there are linear functions $\beta_{r}$ on $\mathbb{A}(X / \Sigma)$ such that

$$
{ }^{+} D_{X}^{w}\left(e^{t S} z\right)=\exp \left(\frac{n t^{2}}{2}\right) \sum_{r=0}^{a} \beta_{r}(z) \cosh (r t) .
$$

The sum only runs over even $r$. Combining this with the previous formula, we find that there are constants $\gamma_{r}$, such that

$$
{ }^{+} R(1)(z)=\sum_{r=0}^{a} \gamma_{r} \beta_{r}(z)
$$


Here the $\beta_{r}$ depend on $(X, \Sigma)$, but the $\gamma_{r}$ depend only on $n, g$ and $w \cdot S$ $\bmod 2$. We will obtain some information about $\gamma_{r}$ from our standard example, $K 3$.

Let $X$ be $K 3$ blown up at $N$ points, and let $\Sigma_{0}, \Sigma_{2}, \ldots, \Sigma_{a}$ be the surfaces of the previous subsection. Because we know the invariants of $X$, we know what $\beta_{r}$ is, for each of the pairs $\left(X, \Sigma_{p}\right)$. In particular, for the pair $\left(X, \Sigma_{p}\right)$, the function $\beta_{r}$ is zero for $r>p$ and is non-zero for $r=p$. On the other hand, in section 5 of [25] it was shown that ${ }^{+} R(1)$ is zero if $\Sigma$ has the form $\Sigma^{\prime} \# T^{2} \# T^{2}$ in $X$. This applies to all $\Sigma_{p}$ for $p \leq a-4$. From this we deduce that $\gamma_{r}=0$ for all $r \leq a-4$. We can also show that $\gamma_{a-2}$ vanishes. To do this, let $\Sigma^{\prime}$ be a tight surface in $K 3$ with genus $g$, and let $\Sigma$ be the following surface with genus $g$ and $a(\Sigma)=a$ :

$$
\Sigma=\Sigma^{\prime} \#\left(C_{1} \# C_{2} \# \cdots \# C_{a-4}\right) \# O_{a-3} .
$$

Here $C_{i}$, as before, is the exceptional curve in the $i$ th blow-up, and $O_{a-3}$ is a sphere representing twice the exceptional class in the $(a-3)$ th blow-up. One of the basic classes, $E_{1}+\cdots+E_{a-3}$, now has intersection number $a-2$ with $\Sigma$. So for this pair $(X, \Sigma)$, we have $\beta_{a-2} \neq 0$ and $\beta_{a}=0$. The results of [25] again give ${ }^{+} R(1)=0$, because $\Sigma$ contains the conic curve $O$ as a summand. We conclude that $\gamma_{a-2}$ is zero.

We have proved that $\gamma_{r}$ is zero for $r<a$. We cannot have $\gamma_{a}=0$ too, because of the observation above about the leading coefficient of $\eta(1, S)$. So there is a non-zero coefficient $\gamma_{a}$ such that

$$
{ }^{+} R(1)(z)=\gamma_{a} \beta_{a}(z) .
$$

The function $\beta_{a}$ is non-zero on $\mathbb{A}(X / \Sigma)$ if and only if there is a basic class $K$ with $K \cdot S=a$. This proves the first statement in the lemma.

The more general statement, about the order of vanishing at $s=1$, has a proof along the same lines, using the fact from [25], that if $\Sigma$ can be written as $\Sigma^{\prime} \#(2 l) T^{2} \# m O$, then the order of vanishing of ${ }^{+} R(s)$ at $s=1$ is at least $2 l+m$.

Lemma 9.4. With the same hypotheses, $R(s)$ has the same order of vanishing at $s=1$ as ${ }^{+} R(s)$.

Proof. We can run through the same arguments with ${ }^{-} R$ as with ${ }^{+} R$, to see that the previous Lemma holds, with the same basic classes. So ${ }^{+} R$ and ${ }^{-} R$ have the same order of vanishing at $s=1$. We have

$$
R(s)={ }^{+} R(s)+{ }^{-} R(s),
$$


so the only way the present Lemma can fail is if there is cancellation on the right hand side. However, for any class $h$ in $[\Sigma]^{\perp} \subset H_{2}(X)$, we have a formula of the general shape

$$
{ }^{+} R\left(e^{t h}\right)=\exp \left(\frac{t^{2} Q(h)}{2}\right) \sum_{\lambda \in \mathbb{Z}} a_{\lambda} e^{\lambda t},
$$

while for ${ }^{-} R$ we have

$$
{ }^{-} R\left(e^{t h}\right)=\exp \left(\frac{-t^{2} Q(h)}{2}\right) \sum_{\lambda \in \mathbb{Z}} b_{\lambda} e^{i \lambda t},
$$

so cancellation is impossible. q.e.d.

In [25] it was shown that, if $X$ is simply connected, the value of ${ }^{+} R_{X, \Sigma}(s)$ at $s=1$ is an obstruction to finding a surface $\Sigma^{\prime}$ of odd genus smaller than $g(\Sigma)$, in the same homology class. More generally, it was shown that any any odd-genus Riemann surface $\Sigma^{\prime}$ in the same homology class as $\Sigma$ must satisfy

$$
2 \text { genus }\left(\Sigma^{\prime}\right)-2 \geq Q(S)+J,
$$

where

$$
J=a(\Sigma)-\text { ord, }
$$

and the last term is the order of vanishing of ${ }^{+} R(s)$ (or just $R(s)$, by the above Lemma) at $s=1$. It was shown that $J$ actually depends only on the homology class $S$, and not on the chosen representative $\Sigma$, as long as $X$ is simply connected.

What the last two lemmas show is that, if $Q(S)$ is sufficiently large and the parities are correct, then

$$
J(S)=\max _{K} K \cdot S,
$$

where $K$ runs over the basic classes. In particular, this holds for all sufficiently large, even multiples of any class of positive square. For such classes, the lower bound (9.5) for the genus, from [25], coincides with the lower bound given by part (b) of Theorem 1.7. Note that the definition of $J$ does not reveal any clues as to the nature of this function of $S$. The lemmas show that it is eventually linear on every ray in the positive cone. It seems natural, therefore to pose the following question: 
Question 1. Let $X$ be a simply-connected 4-manifold of simple type. Let $J$ be the function on $H_{2}(X ; \mathbb{Z})$ defined by the order of vanishing of the series $R(s)$ at $s=1$, as above. Is $J(S)$ equal to $\max _{K}(K \cdot S)$ for all classes $S$ of positive square? What about classes of negative square?

A related question is whether the ubiquitous assumption (for example in (5.10)) that $n$ and $g$ are large compared to $a(\Sigma)$ can be relaxed. A glance at the formulae for the elements $\omega_{p}^{w}=\omega_{p}^{w}(n, g)$ given in section 6 shows that their coefficients have a polynomial dependence on $n$ and $g$, at least for $p \leq 4$. This is likely to be a general phenomenon, as there are recurrence formulae relating the $\omega_{p}^{w}$ for different values of $n$ and $g$ (a matter which the authors hope to take up in a later paper). We can therefore extrapolate downwards, so as to define a class $\omega_{p}^{w}(n, g)$ even when $\Delta(n, g)<2 p$.

Question 2. Let $\omega_{p}^{w}(n, g)$ be defined when $\Delta(n, g)<2 p$ by extrapolating the formulae from large $n$ and $g$. Does the universal formula for $D_{X, \Sigma}^{w, p}$ given in Theorem 5.10 continue to hold in this range?

(iii) Complex surfaces and related topics. Regular algebraic surfaces with non-zero geometric genus $p_{g}$ are 4-manifolds to which our main theorem may apply, though we do not know whether they all have simple type. If $X$ is such a surface and does have simple type, we can sometimes prove that its canonical class $K_{X}$ is one of the basic classes, using the two lemmas of the previous subsection.

Let $X$ be such a surface and let $\Sigma$ be a smooth, complex curve with large, even self-intersection and odd genus. We can take $\Sigma$ to belong to the linear system of some large, even multiple of the hyperplane class of some projective embedding. Because it is complex, the genus of $\Sigma$ is given by the adjunction formula, so $a(\Sigma)=K_{X} \cdot \Sigma$. In [24], a non-vanishing theorem was stated; the Theorem extends an argument from [38] to show that, for the pair $(X, \Sigma)$, the value of $R(s)$ at $s=1$ is non-zero provided that $p_{g}(X)$ is odd and that there is a smooth, reduced divisor in the canonical linear system of the minimal model. (It has since been pointed out to the authors that the assumption of smoothness can probably be eliminated using the results of [30].) From the previous lemmas, then, there is a basic class $K$ such that

$$
K \cdot \Sigma=K_{X} \cdot \Sigma \text {. }
$$

In [6] it is shown that this equality can hold only if $K=K_{X}$. We therefore have: 
Proposition 9.6. Let $X$ be a regular algebraic surface with $p_{g}$ odd. Suppose $X$ is of simple type and has a smooth, reduced canonical divisor. Then the canonical class of $X$ is one of the basic classes.

Question 3. Let $X$ be a regular algebraic surface of simple type. Is the canonical class $K_{X}$ always a basic class? What about symplectic 4-manifolds, where one defines $K_{X}$ as minus the first Chern class of a compatible almost complex structure?

The techniques of [15] have recently allowed calculation of the invariants of some regular surfaces. Since the elliptic case is well-understood now, and the effect of a blow-up is known, the remaining questions of calculation concern minimal surfaces of general type. In all cases where the invariant of a minimal, regular surface $X$ of general type has been calculated, the result has been that $X$ has simple type, the only basic classes are $\pm K_{X}$, and the coefficient $\beta$ of $e^{K_{X}}$ in the formula of Theorem 1.7 is

$$
\beta=2^{K_{X}^{2}-\chi(\mathcal{O})+2},
$$

where $\chi(\mathcal{O})=p_{g}+1$ is the holomorphic Euler characteristic, so

$$
\mathscr{D}_{X}^{w}=2^{K_{X}^{2}-\chi(\mathcal{O})+2} \exp \left(\frac{Q}{2}\right)\left(e^{K_{X}} \pm e^{-K_{X}}\right) .
$$

This prompts the following question, which subsumes part of Question 3.

Question 4. Is the formula (9.7) correct for all (perhaps regular) minimal surfaces of general type with $p_{g}>0$ ? In particular, do such 4-manifolds always have simple type?

It may be that one obtains the result above in all known cases only because one can always use the constructions of [15] to make the calculation, and these constructions preserve this form of the answer. However, some weight has been added to the speculation that (9.7) might hold generally: recent work of Witten [43] shows that a formula equivalent to this can be derived from standard conjectures using the procedures of quantum field theory, at least in the case that $X$ has a smooth, reduced canonical divisor.

In all known examples, the basic classes $K$ satisfy $K^{2}=2 e+3 \sigma$, where $e$ is the Euler number and $\sigma$ is the signature. These examples include some non-Kähler symplectic manifolds with $K^{2}$ non-zero.

Question 5. If $X$ is a 4-manifold of simple type with $b^{1}=0$, do the basic classes $K$ always satisfy $K^{2}=2 e+3 \sigma$ ? 
Finally, we ask:

Question 6. Is the lower bound (1.7)(b) for the genus of embedded surfaces sharp, in the case of classes of positive square in a 4-manifold $X$ of simple type with $\mathscr{D}_{X}^{w}$ non-zero? If not, is it at least sharp for large enough multiples of a fixed class in the positive cone?

The first five questions, though some may require substantial new ideas, appear to be within the possible scope of gauge theory. It is clear, however, that to give an affirmative answer to Question 6 one would need to draw on quite different techniques.

\section{Appendix: The orientation of moduli spaces}

The purpose of this appendix is to describe conventions for consistently orienting the moduli spaces on manifolds with cylindrical ends that we encounter, as well as moduli spaces of singular connections.

Let $X$ be an oriented 4-manifold with a homology-orientation, and $\Sigma$ an oriented embedded surface of genus $g$. Let $w$ be a line-bundle on $X$, and use the same letter to denote the restriction of the line-bundle to $X^{o}$. On $X^{o}$, the line-bundle has a preferred cylindrical-end structure, and $\left.w\right|_{Y}$ is given as pulled-back from $\Sigma$. Fix a compact submanifold with boundary, $\mathscr{R}^{\sim} \subset \mathscr{R}_{\mathrm{ev}}^{w, s}$, of dimension $6 g-6$. Let $\mathscr{B}_{\kappa}^{*, w}\left(X^{o} ; \mathscr{R}^{\sim}\right)$ be the Banach manifold consisting of irreducible connections on the cylindrical-end manifold $X^{o}=X / \Sigma$ having $\delta$-exponential decay to a flat connection $\rho \in \mathscr{R}^{\sim}$ (for some sufficiently small $\delta$, depending on $\left.\mathscr{R}^{\sim}\right)$, as described in [36]. Let $\lambda\left(X_{0}\right) \rightarrow \mathscr{B}_{\kappa}^{*, w}\left(X^{o} ; \mathscr{R}^{\sim}\right)$ be the real determinant line bundle corresponding to the deformation complex $E_{\delta}$ of [36]. It follows from [36] and the fact that $\mathscr{R}^{\sim}$ is orientable, that $\lambda\left(X^{o}\right)$ is orientable. To orient the moduli spaces $M_{\kappa}^{w}\left(X^{o} ; \mathscr{R}_{\mathrm{ev}}^{w, s}\right)$, we need to specify a convention for orienting $\lambda\left(X^{\circ}\right)$.

Let $\lambda(X)$ denote the real determinant line bundle over $\mathscr{B}_{\kappa}^{w}(X)$, for the deformation operator on the closed manifold. By the excision principle, if specify an orientation for the quotient

$$
\lambda\left(X^{o}\right) \otimes \lambda(X)^{-1},
$$

then this will determine an orientation for the same quotient in all cases where the pair $\left(\Sigma,\left.w\right|_{\Sigma}\right)$ is isomorphic. Consider therefore the closed ruled surface $X=W$ containing the surface $\Sigma=\Sigma_{+}$. Let $w$ be the 
line-bundle on $X$ pulled back from $\Sigma$. Let $X^{o}=X / \Sigma$. In this instance, the moduli spaces $M_{0}^{w}(X)$ and $M_{0}^{w}\left(X^{o} ; \mathscr{R}_{\mathrm{ev}}^{w}\right)$ are both isomorphic to the representation variety $\mathscr{R}_{\mathrm{ev}}^{w}$, and we orient the above quotient by the canonical trivialization which results. A homology orientation, $o$, for the closed manifold $X$ determines an orientation for $\lambda(X)$ by [9]. Having trivialized the quotient, we therefore obtain an orientation for $\lambda\left(X^{o}\right)$.

There is another useful way of looking at this. Let $\mathscr{B}_{\kappa}^{w, *}\left(W^{o} ; \rho\right)$ be the Banach manifold of irreducible connections on $W^{o}$ asymptotic to a fixed $\rho \in \mathscr{R}_{\mathrm{ev}}^{w, s}$. Let $\lambda^{f}\left(W^{o}\right)$ be the determinant line for the complex $F(\delta)$ of [36] on $W^{o}$. An orientation of $\lambda^{f}\left(W^{o}\right)$ determines an orientation of the fibres of the boundary map $r: M^{w}\left(W^{o} ; \mathscr{R}_{\mathrm{ev}}^{w}\right) \rightarrow \mathscr{R}_{\mathrm{ev}}^{w}$ over smooth points, when $r$ is a submersion, and (equivalently) determines an orientation of the normal bundle of $r$ where $r$ is an immersion. A choice of orientations for $\lambda\left(X^{o}\right)$ and $\lambda^{f}\left(W^{o}\right)$ determine an orientation for $\lambda(X)$, via the gluing description of the $M^{w}(X)$, as a fibred product. Our chosen orientation for the quotient (A.1) above corresponds to the canonical orientation for the moduli space $M_{0}^{w}\left(W^{o} ; \rho\right)$, which is a single point.

For the odd component, one can fix an orientation for $M_{\kappa}^{w}\left(X^{o} ; \mathscr{R}^{w, s}\right)$ by identifying it with $M_{\kappa}^{w^{\prime}}\left(X^{o} ; \mathscr{R}_{\mathrm{ev}}^{w^{\prime}, s}\right)$, where $w^{\prime}=w-[\Sigma]$.

We now turn to the moduli spaces of singular connections. In this situation, we have determinant line bundles $\lambda(X, \Sigma), \lambda^{f}\left(W^{o}, \Sigma\right)$ and $\lambda\left(X^{o}\right)$. Trivializations of these determine orientations of the moduli spaces $M_{\kappa}^{w, p, \alpha}(X, \Sigma), M_{\kappa}^{w, p, \alpha}\left(W^{o}, \Sigma ; \mathscr{R}_{\mathrm{ev}}^{w, s}\right)$ and $M_{\kappa}^{w}\left(X^{o} ; \mathscr{R}_{\mathrm{ev}}^{w, s}\right)$ respectively. From [27], a homology orientation of $X$ determines an orientation of $\lambda(X, \Sigma)$. From the previous paragraphs, we have an orientation of $\lambda\left(X^{o}\right)$. These two therefore determine an orientation of $\lambda^{f}\left(W^{o}, \Sigma\right)$, compatible with the fibre-product description.

Let us spell out the consequences of this prescription. The excision principal assures us that the orientation of $\lambda^{f}\left(W^{o}, \Sigma\right)$ which we have just specified is independent of $X$. We therefore take $X$ to be the closed ruled surface. The moduli space $M_{0}^{w, *}(X)=M_{0}^{w}\left(X^{o} ; \mathscr{R}_{\mathrm{ev}}^{w, s}\right)$ is a complex manifold, and is isomorphic to the representation variety. The standard orientation of this moduli space [9] differs from the complex orientation by the sign

$$
(-1)^{\left(w^{2}+K_{X} \cdot w\right) / 2}=(-1)^{w \cdot \Sigma}
$$


The moduli space $M_{0}^{w, p, \alpha}(X, \Sigma)$ is complex too, and its standard orientation [27] differs from its complex orientation by the same sign. It follows that our chosen orientation of $M^{w, p, \alpha}\left(W^{o}, \Sigma ; \rho\right)$ is the one which gives the complex orientation to the fibres of

$$
r: M_{\kappa}^{w, p, \alpha}\left(W^{o}, \Sigma ; \mathscr{R}_{\mathrm{ev}}^{w, s}\right) \rightarrow \mathscr{R}_{\mathrm{ev}}^{w, s},
$$

(or the complex orientation to the normal, as the case may be).

The only remaining task is to determine whether the standard orientations are preserved by the 'flip' identification

$$
\Phi: M_{\kappa}^{w, p, \alpha}(X, \Sigma) \rightarrow M_{\kappa^{\prime}}^{w^{\prime}, p^{\prime}, \alpha^{\prime}}(X, \Sigma),
$$

as discussed at the beginning of section 7 . It is enough to consider the case that $X$ is the closed ruled surface. In this case, the moduli spaces are all complex, and $\Phi$ is holomorphic, so it preserves the complex orientation. The standard orientation of $M_{\kappa}^{w, p, \alpha}$ differs from the complex orientation by the sign

$$
(-1)^{\left(w^{2}+K_{X} \cdot w\right) / 2}=(-1)^{w} \cdot \Sigma,
$$

where we have used the equality $K_{X}=-2\left[\Sigma_{-}\right]+(2 g-2-n)[F]$ in $H^{2}(X)$. (Here $n=Q([\Sigma])$.) Similarly, the orientation of $M_{\kappa^{\prime}}^{w^{\prime}, p^{\prime}, \alpha^{\prime}}$ differs from the complex orientation by

$$
(-1)^{\left(\left(w^{\prime}\right)^{2}+K_{X} \cdot w^{\prime}\right) / 2}=(-1)^{g-1-n},
$$

as $w^{\prime}=w-[\Sigma]$. Therefore $\Phi$ preserves or reverses the standard orientations according to the parity of $w \cdot \Sigma+g-1-n$. This is the same as the parity of $p^{\prime}=a(\Sigma)-p$, because $p$ satisfies the parity condition (2.20) and $a(\Sigma)=n \bmod 2$.

\section{References}

[1] E. Arbarello, M. Cornalba, P.A. Griffiths \& J. Harris, Geometry of Algebraic Curves, I, Grundlehren der math. Wissenschaften 267, Springer-Verlag, New York, 1985.

[2] M.F. Atiyah \& R. Bott, The Yang-Mills equations over Riemann surfaces, Phil. Trans. R. Soc. Lond. A 308 (1982), 523-615. 
[3] M.F. Atiyah, V.K. Patodi \& I.M. Singer, Spectral asymmetry in Riemannian geometry, I, Math. Proc. Camb. Phil. Soc. 77 (1975a) 43-69.

[4] S. Bando, Einstein-Hermitian metrics on non-compact Kähler manifolds, (preprint).

[5] O. Biquard, Sur les fibrés paraboliques sur une surface complexe, (preprint).

[6] R. Brussee, Some remarks on the Kronheimer-Mrowka classes of algebraic surfaces, (preprint).

[7] N.P. Buchdahl, Hermitian-Einstein connections and stable vector bundles over compact complex surfaces, Math. Ann. 280 (1988) 625-648.

[8] S.K. Donaldson, Connections, cohomology and the intersection forms of four-manifolds, J. Differential Geometry 24 (1986) 275341.

[9] $\longrightarrow$ The orientation of Yang-Mills moduli spaces and 4-manifold topology, J. Differential Geometry 26 (1987) 397-428.

[10] S.K. Donaldson, Polynomial invariants for smooth four-manifolds, Topology 29 (1990) 257-315.

[11] S.K. Donaldson \& P.B. Kronheimer, The geometry of four-manifolds, Oxford University Press (1990).

[12] J.L. Evans, D.Phil. qualifying dissertation, Oxford 1992 (unpublished).

[13] R. Fintushel \& R.J. Stern, The blowup formula for Donaldson invariants, (preprint).

[14] — Donaldson invariants of 4-manifolds with simple type, (in preparation)

[15] — Rational blowdowns of smooth 4-manifolds, (in preparation)

[16] D.S. Freed \& K.K. Uhlenbeck, Instantons and four-manifolds, M.S.R.I. Publications, Vol. 1, (Springer-Verlag, New York, 1984).

[17] R. Friedman \& J.W. Morgan, Smooth 4-manifolds and Complex Surfaces, Ergebnisse der Math. und Grenz. 3, (Springer-Verlag, New York, 1994). 
[18] R.E. Gompf, Three exotic $\mathbb{R}^{4} s$ and other anomolies, J. Differential Geometry 18 (1983) 317-28.

[19] R.E. Gompf \& T.S. Mrowka, Irreducible 4-manifolds need not be complex, Ann. Math. 138 (1993) 61-111.

[20] G.-Y. Guo, Differential geometry of complex vector bundles on non-compact manifolds, (D.Phil. thesis, Oxford, 1993).

[21] H.-J. Hoppe \& H. Spindler, Modulraume stabiler 2-Bundel auf Regelflachen, Math. Ann. 249 (1980) 127-40.

[22] J.C. Hurtubise \& R.J. Milgram, The Atiyah-Jones conjecture for ruled surfaces, (preprint).

[23] D. Kotschick, $S O(3)$ invariants for 4-manifolds with $b_{2}^{+}=1$, Proc. London Math. Soc. 63 (1991) 426-48.

[24] P.B. Kronheimer, The genus-minimizing property of algebraic curves, Bull. Amer. Math. Soc. 29 (1993) 63-9.

[25] _ An obstruction to removing intersection points in immersed surfaces, Topology (to appear).

[26] P.B. Kronheimer \& T.S. Mrowka, Gauge theory for embedded surfaces: I, Topology 32 (1993) 773-826.

[27] — Gauge theory for embedded surfaces: II, Topology 34 (1995) 37-97.

[28] _ Recurrence relations and asymptotics for four-manifold invariants, Bull. Amer. Math. Soc. 30 (1994) 215-21.

[29] H.B. Jr. Lawson, The theory of gauge fields in four dimensions, CBMS Regional Conference Series in Mathematics, 58, American Mathematical Society, Providence, R.I. (1985).

[30] J. Li, Kodaira dimension of moduli space of vector bundles on algebraic surfaces, Inventiones Mathematicae 115 (1994) 1-40.

[31] P. Lisca, Computation of instanton invariants using DonaldsonFloer theory, (preprint).

[32] R. Lockhart \& R. McOwen, Elliptic differential operators on noncompact manifolds, Annali di Scoula Norm. Sup. de Pisa, IV-12 (1985) 409-48.

[33] T. Matumoto, On diffeomorphisms of a K3 surface, (Hiroshima University Reprint, 1985). 
[34] J.W. Morgan, T.S. Mrowka, A note on Donaldson's polynomial invariants, Int. Math. Research Notices 10 (1992) 223-30.

[35] — On the gluing theorem for instantons on manifolds with long tubes, (in preparation).

[36] J.W. Morgan, T.S. Mrowka \& D. Ruberman, The $L^{2}$ moduli space and a vanishing theorem for Donaldson's polynomial invariants, Monographs in Geometry and Topology 2, (International Press, 1994).

[37] K.G. O'Grady, Donaldson's polynomials for K3 surfaces, J. Differential Geometry 35 (1992) 415-27.

[38] — Algebro-geometric analogues of Donaldson's polynomials, Inventiones Mathematicae 107 (1992) 351-395.

[39] C.S. Seshadri, Space of unitary vector bundles on a compact Riemann surface, Ann. Math. 85 (1967) 303-36.

[40] C.H. Taubes, Gauge theory on asymptotically periodic 4-manifolds, J. Differential Geometry 17 (1987) 363-430.

[41] $\longrightarrow, L^{2}$ moduli spaces on manifolds with cylindrical ends, Monographs in Geometry and Topology 1, (International Press, 1994).

[42] — Donaldson-Floer theory for circle bundles over Riemann surfaces, (in preparation).

[43] E. Witten, Supersymmetric Yang-Mills theory on a four-manifold, (preprint).

OXFORD UNIVERSITY, OXFORD, ENGLAND California Institute of TeChNology 